Florida International University

FIU Digital Commons

8-24-2018

\title{
Forensic Toxicological Screening and Confirmation of 800+ Novel Psychoactive Substances by LC-QTOF-MS and 2D-LC Analysis
}

\author{
Melanie N. Eckberg \\ Florida International University, meckb001@fiu.edu
}

Follow this and additional works at: https://digitalcommons.fiu.edu/etd

Part of the Analytical Chemistry Commons, and the Other Chemistry Commons

\section{Recommended Citation \\ Eckberg, Melanie N., "Forensic Toxicological Screening and Confirmation of 800+ Novel Psychoactive Substances by LC-QTOF-MS and 2D-LC Analysis" (2018). FIU Electronic Theses and Dissertations. 3923. https://digitalcommons.fiu.edu/etd/3923}

This work is brought to you for free and open access by the University Graduate School at FIU Digital Commons. It has been accepted for inclusion in FIU Electronic Theses and Dissertations by an authorized administrator of FIU Digital Commons. For more information, please contact dcc@fiu.edu. 


\title{
FLORIDA INTERNATIONAL UNIVERSITY \\ Miami, Florida
}

FORENSIC TOXICOLOGICAL SCREENING AND CONFIRMATION OF 800+ NOVEL PSYCHOACTIVE SUBSTANCES BY LC-QTOF-MS AND 2D-LC ANALYSIS.

A dissertation submitted in partial fulfillment of

\author{
the requirements for the degree of \\ DOCTOR OF PHILOSOPHY \\ in \\ CHEMISTRY \\ By
}

Melanie Nicole Eckberg 
To: Dean Michael R. Heithaus

College of Arts, Sciences, and Education

This dissertation, written by Melanie Nicole Eckberg, and entitled Forensic Toxicological Screening and Confirmation of 800+ Novel Psychoactive Substances by LC-QTOF-MS and 2D-LC Analysis, having been approved in respect to style and intellectual content, is referred to you for judgment.

We have read this dissertation and recommend that it be approved.

Jose Almirall

Yong Cai

Georg Petroianu

Stanislaw Wnuk

Anthony DeCaprio, Major Professor

Date of Defense: August 24, 2018

The dissertation of Melanie Nicole Eckberg is approved.

Dean Michael R. Heithaus

College of Arts, Sciences, and Education

Andrés G. Gil

Vice President for Research and Economic Development and Dean of the University Graduate School

Florida International University, 2018 
(C) Copyright 2018 by Melanie Nicole Eckberg

All rights reserved. 


\section{DEDICATION}

This dissertation is dedicated to my friends and family, especially my parents, without whom, none of this would have been possible. Thank you for all of your support, your patience, and your love. I am excited to close this chapter of my life and begin the next with all of you by my side.

“For last year's words belong to last year's language and next year's words await another voice. And to make an end is to make a beginning."

-T.S. Eliot 


\section{ACKNOWLEDGMENTS}

First, I wish to express my gratitude to Dr. Anthony DeCaprio for serving as my research advisor and allowing me to be a part of his research laboratory. I would like to thank Dr. José Almirall, Dr. Yong Cai, Dr. Stanislaw Wnuk, and Dr. Georg Petroianu for serving as my committee members. I am extremely grateful for your constructive criticism, words of advice, and willingness to help me become a better scientist. Thanks to Dr. Luis Arroyo for introducing me to the QTOF and helping me to learn most of what I know about that instrumentation. Special thanks to Ralph Hindle for your invaluable assistance and guidance throughout all of my work and for teaching me a lot about everything. I also wish to thank Dr. Dwight Stoll of Gustavus Adolphus College for welcoming me to his laboratory for a week in order to teach me the basics of 2D-LC and help in my preliminary data acquisition. Without your help, my $2 \mathrm{D}$ project would have never gotten off the ground.

Words cannot express how grateful I am to my friends and family for coming with me on this journey. I could not have made it through these past five years without all of you. When I came to Miami I had no idea what I was in for. I am so lucky to have found a family here that loves and supports me and can understand what I'm going through. Vanessa (aka V\$), you were the first to really welcome me into the lab and although we only shared that space for a few months, you quickly became one of the most important people in my life. Thank you for always being so real and for keeping me grounded and for letting me be a part of your family (kthxluvyoubye). Erika, I literally could not have done this without you. I am so happy to have been adopted into your life. 
You've kept me sane (and well fed) and were always there for me to talk to. You and Mitch have truly become family and I am so excited to be Auntie Mel from now until forever (love you J!). James, what would I ever do without you? I'm so lucky to have found another brother that gets me and shares in my love of all things pop culture. Thank you for being my outlet when school got tough and I needed a break. I look forward to many more years of Disney adventures, personalized theme park plans and guides, Harry Potter madness, all the sports, geeking out over movies and TV, going to cons, and so, so much more! To W.E., thank you for helping me learn to accept my flaws and embrace them as sources of strength. You have shown me that I don't have to be perfect to do great things and helped me to truly appreciate the value of my Found Family. Haley, you have been such a great support even as you were going through your own grad school experience. Thank you for being my link to home and to the Ramily and I can't wait to be that support for you! Joanna. You have literally been there since Day One all the way back in our dorms at VCU. Even though we have been thousands of miles apart as you've undergone your own $\mathrm{PhD}$ adventure, your unwavering love and support has always been felt. Who knew that we would both end up on this journey and I can't imagine the last nine years without you! D.H., look at where we are, look at where we started. You have been such a source of strength and support for so many years. I definitely could not have kept any amount of sanity without your friendship. Thank you for always being there for me and for always politely nodding and listening to my science woes. I don't think I would have been able to grow as a person as much as I have without you and I look forward to so many more (non-science) adventures! 
To my lab family, I'm not sure how I will get through the days without you. Allen, we started this journey together and I am so lucky to have had your unwavering support throughout the last five years. Your humor, your advice, your candor, and your willingness to always help have gotten me through more days than you may realize and I will always treasure our Taco Bell visits. Ashley, you were our first "junior" student but have become much more of a partner in the lab and outside of it. Your friendship has meant so much to me and I know for sure that I definitely could not have gotten to this point without you. Jenna, to say that you have made my life interesting is a vast understatement but I wouldn't change any of it. You've been a great sounding board and support for me and I am really going to miss our coffee runs. Josh, thank you so much for teaching me how to do almost everything in the lab. Even though we may not agree on our sports teams, I guess you are a pretty good friend and I know I will be learning from you for years to come. Quentin, you may not officially be part of the lab, but we might as well have given you access for all the time you spend there. Thank you for being another voice of reason amongst our group, and for making sure I was having fun and relaxing. I will never forget all of our adventures.

Kat, when we first met back at Visitation, I knew I would be fortunate to have your friendship. The last $5+$ years have never been dull and I am so glad that we got assigned to room together to start with. I will never forget our adventures of living in Doral together and all of our years of friendship after that. Thad, thank you for always being another sounding board for me and for being another "adult" here. I am so grateful for your help whenever I asked and for your friendship throughout. 
To my FIU family, thank you for your incredible support! I absolutely would never have been able to get through any of these years without you. Maggie, you were always there to help me navigate FIU and the Department and I am forever grateful for your help. Pupi, you have been so supportive and helpful and I am going to miss you and your chats so much. Jackie, thank you for always being another resource for me to go to, but also someone I could vent with and call a friend.

To my family, thank you so much for all of your little shows of support. From the little postcards, to the letters, to the text messages sent every now and then, these little bits of love were extremely appreciated and helped pick me up when I really needed an extra boost.

To my mom and dad, your unwavering love and support (and attempts at understanding when I vented to you about science) helped keep me going more than you could ever imagine. You have always been there for me and even more so during my time in Miami when I have had some of my highest highs and my lowest lows. You have sacrificed so much for me to get to this point and those sacrifices and efforts do not go unnoticed or unappreciated. This dissertation is for me, but also belongs to you. I never would have gotten this far without you and I'm proud to call you my parents. Nick, I could not be more proud of you or more grateful to have a little brother like you. We have always been close, but even when we were separated by a continent, I have always felt your love and support and I can't wait to be back in the same state with you.

I love you all and I am so thankful to have had your love and support in my life. Thank you being a part of my journey and this is for you as much as it is for me. 


\title{
ABSTRACT OF THE DISSERTATION
}

\section{FORENSIC TOXICOLOGICAL SCREENING AND CONFIRMATION OF 800+ NOVEL PSYCHOACTIVE SUBSTANCES BY LC-QTOF-MS} AND 2D-LC ANALYSIS

\section{Melanie Nicole Eckberg}

Florida International University, 2018

\author{
Miami, Florida
}

\section{Professor Anthony DeCaprio, Major Professor}

Novel psychoactive substances (NPS) represent a great challenge to toxicologists because of the ability of illicit drug manufacturers to alter NPS chemical structures quickly and with ease to circumvent legislation regulating their use. Each time a new structure is introduced, there is a possibility that it has not been previously recorded in law enforcement or scientific databases. Many toxicology laboratories use targeted analytical methods that rely on libraries of known compounds to identify drugs in samples. However, these libraries do not include large numbers of NPS, which could result in non-identification or detection.

High-resolution mass spectrometry (HRMS) has been suggested as a method for screening a wide variety of analytes because of its high sensitivity and mass accuracy as compared to some other forms of mass spectrometry. The technique can generate characteristic MS/MS spectral data for use in compound identification. The main goal of 
this research was to create a high-resolution mass spectrometry (HRMS) library of NPS and metabolites, as well as to validate a method for screening and confirmation of these substances. The study consisted of three main tasks which included; 1) the development of a large high-resolution MS/MS spectral library and database, 2) validation of a method for screening and confirmation of over 800 NPS and metabolites, and 3) screening of blind-spiked and authentic urine specimens to determine real-world applicability of the HRMS library and method.

During validation, several isomeric and structurally related NPS were observed which could not be adequately separated using traditional LC methods. A fourth task was therefore added to investigate improved separation using two-dimensional liquid chromatography (2D-LC). Increased resolving power is achieved in 2D-LC through the coupling of multiple orthogonal separation systems. Ultimately, an on-line, comprehensive method was developed using orthogonal reversed-phase columns in each dimension (RP $x \mathrm{RP})$ for improved separation of isomeric and structurally similar synthetic cannabinoids.

This work can aid laboratories in the identification of NPS through the use of a validated LC-QTOF-MS method for screening and confirmation and HRMS spectral library. In instances where isomeric and structurally related NPS are not sufficiently separated, RP x RP methods can be explored. 


\section{TABLE OF CONTENTS}

CHAPTER

PAGE

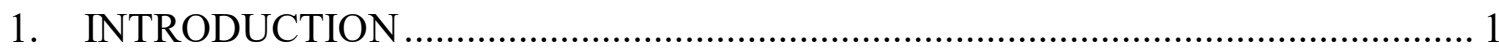

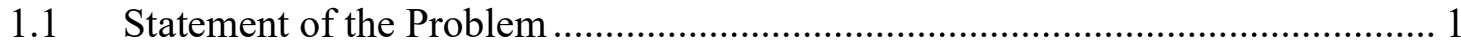

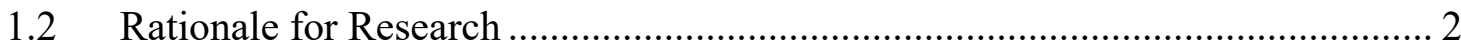

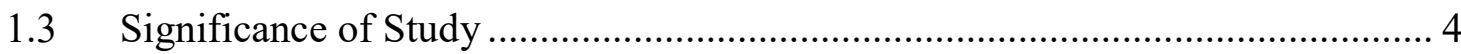

1.3.1 Task 1 - Development of database and spectral library .............................. 4

1.3.2 Task 2-Comprehensive LC-QTOF-MS method validation......................... 5

1.3.3 Task 3 - Investigation of a 2D-LC method ................................................ 5

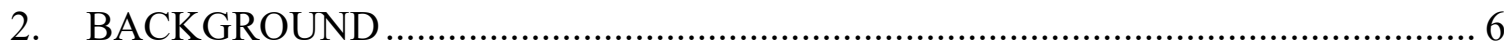

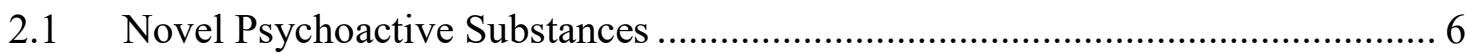

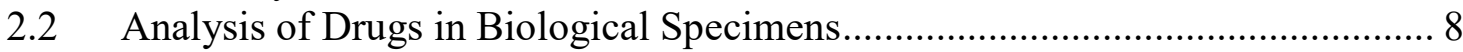

2.3 Liquid Chromatography Mass Spectrometry ……………................................. 13

2.3.1 Electrospray Ionization Spectral Libraries ………………………................ 15

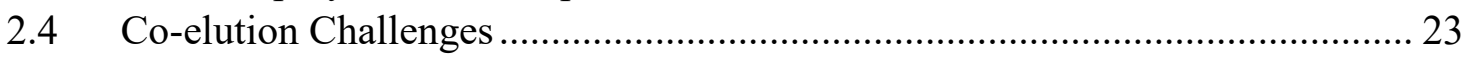

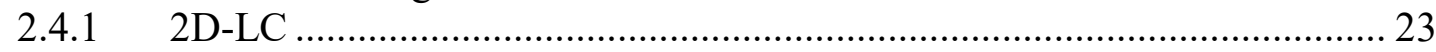

3. DEVELOPMENT OF DATABASE AND SPECTRAL LIBRARY ……………........ 39

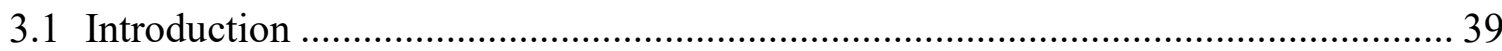

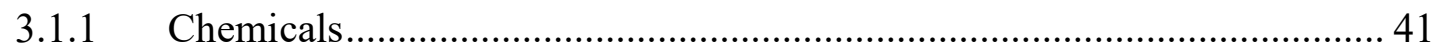

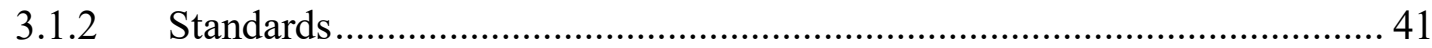

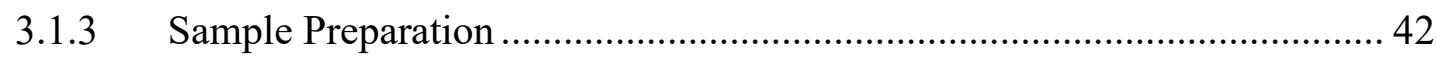

3.1.4 Instrumentation and Software ............................................................. 42

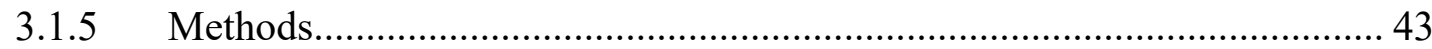

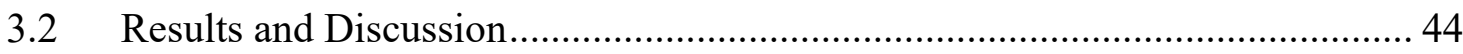

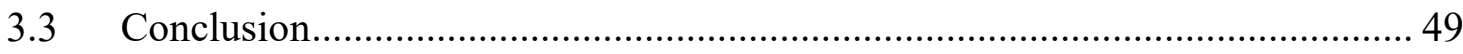

4. COMPREHENSIVE LC-QTOF-MS METHOD VALIDATION ………………......... 51

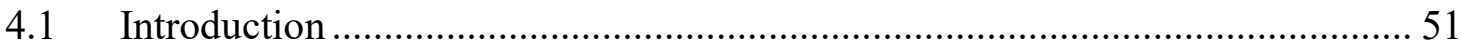

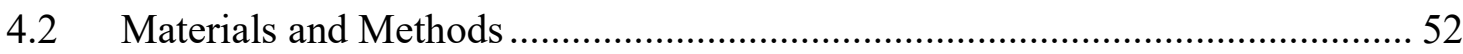

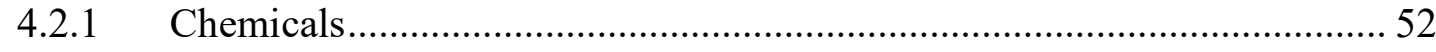

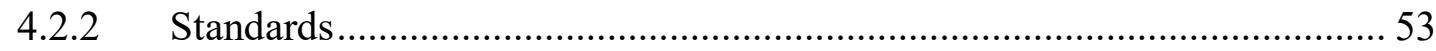

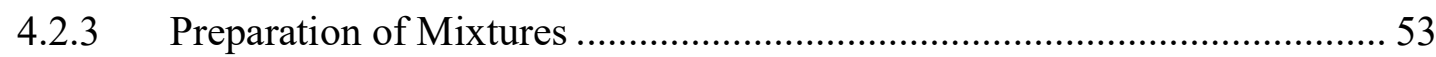

4.2.4 Instrumentation and Software ................................................................. 54

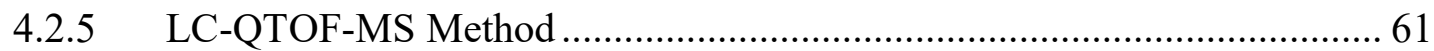

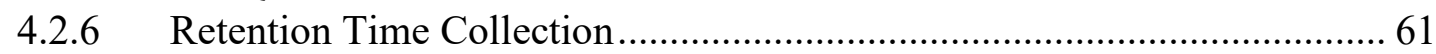

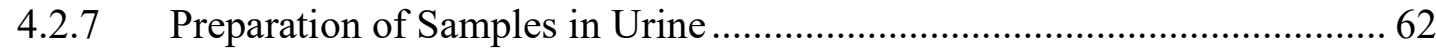

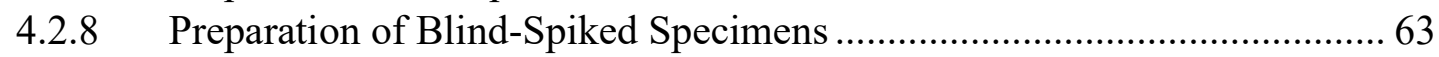

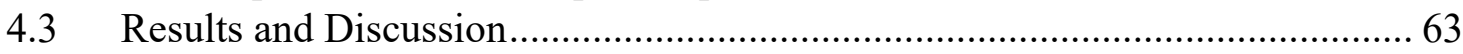

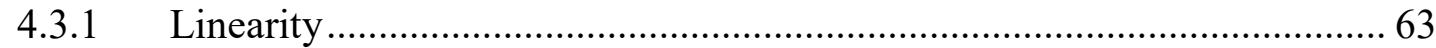

4.3.2 Accuracy and Precision.................................................................................. 64

4.3.3 Limits of Detection and Quantitation .......................................................... 67 


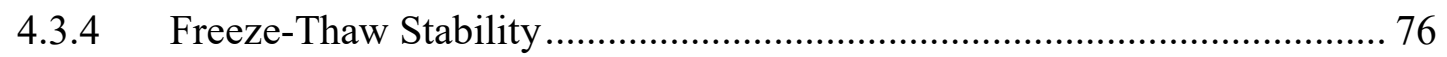

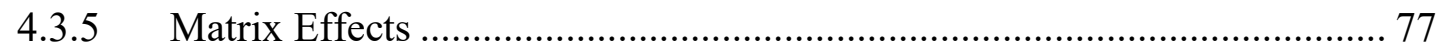

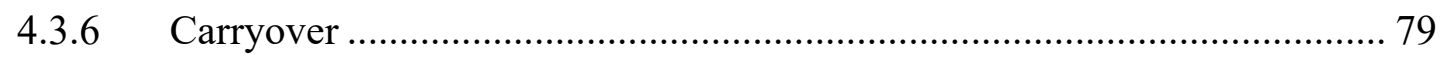

4.3.7 Analysis of Blind-Spiked Specimens....................................................... 80

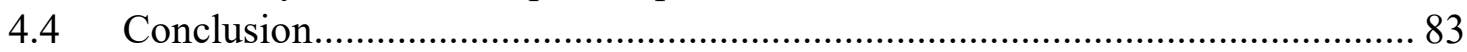

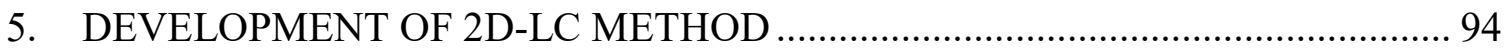

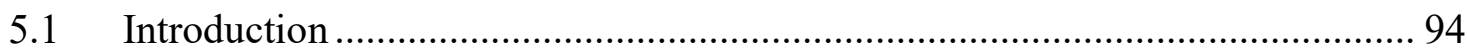

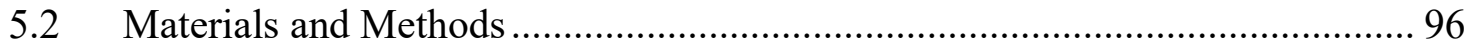

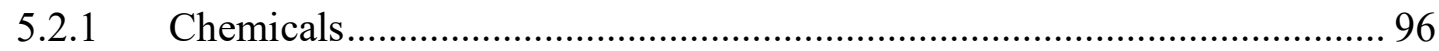

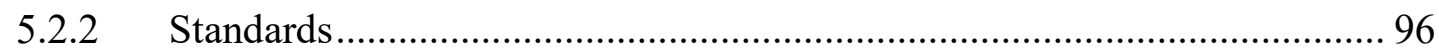

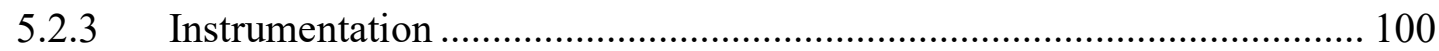

5.2.4 Analytical Columns and Separation...................................................... 102

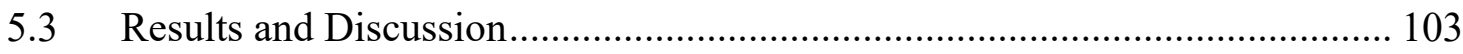

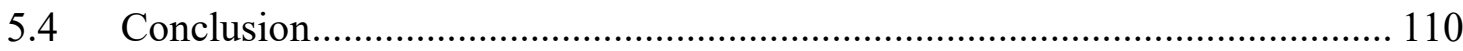

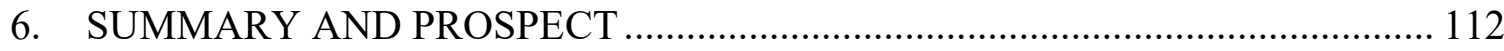

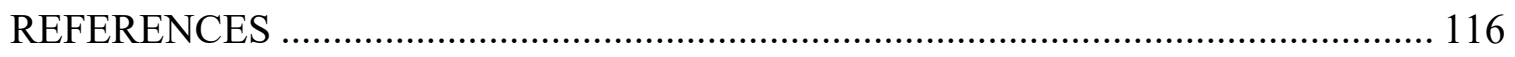

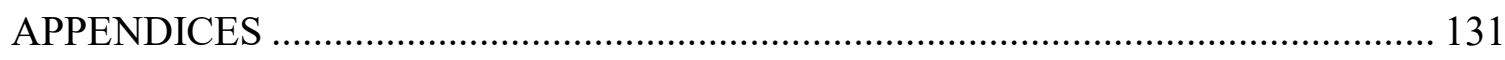

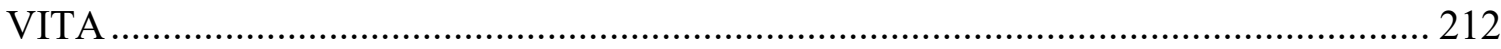




\section{LIST OF TABLES}

TABLE

PAGE

Table 1. Examples of 2D-LC method applications found throughout the literature. 37

Table 2. Distribution of compounds in database by class. 46

Table 3. Distribution of compounds in database by molecule type.

Table 4. Compounds contained in validation Mix 1, their accurate masses, and retention times.

Table 5. Compounds contained in validation Mix 2, their accurate masses, and retention times.

Table 6. Compounds contained in validation Mix 3, their accurate masses, and retention times.

Table 7. Compounds contained in the internal standard (IS) mixture and their accurate masses.

Table 8. Bias and precision data for compounds in Mix 1. Concentrations for low, med, and high are 5, 20, and $80 \mathrm{ng} / \mathrm{mL}$, respectively.

Table 9. Bias and precision data for compounds in Mix 2. Concentrations for low, med, and high are 5,20 , and $80 \mathrm{ng} / \mathrm{mL}$, respectively.

Table 10. Bias and precision data for compounds in Mix 3. Concentrations for low, med, and high are 5, 20, and $80 \mathrm{ng} / \mathrm{mL}$, respectively.

Table 11. LOD and LOQ values for compounds in Mix 1 84

Table 12. LOD and LOQ values for compounds in Mix 2 ................................. 85

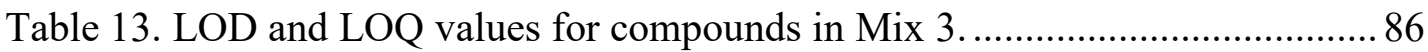

Table 14. Stability data for Mix 2 compounds after three freeze-thaw cycles. ......... 87

Table 15. Stability data for Mix 3 compounds after three freeze-thaw cycles. ......... 88

Table 16. Ion suppression and enhancement (matrix effects) values for compounds in Mix 1.

Table 17. Ion suppression and enhancement (matrix effects) values for compounds in Mix 2. 
Table 18. Ion suppression and enhancement (matrix effects) values for

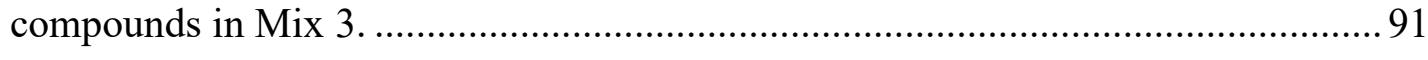

Table 19. Results of blind-spiked urine specimen screening for Set 1. Specimens were prepared at concentrations of 2 or $20 \mathrm{ng} / \mathrm{mL}$................................................. 92

Table 20. Results of blind-spiked urine specimen screening for Set 2. Specimens were prepared at concentrations of $200 \mathrm{ng} / \mathrm{mL}$...................................................... 93

Table 21. Synthetic cannabinoids present in each co-elution (CE) mix.................... 98 


\section{LIST OF FIGURES}

FIGURE

PAGE

Figure 1. Second-dimension gradient types over time for LC x LC separations: A) full gradient, B) segment gradient, C) parallel gradient, and D) shift gradient. ........ 36

Figure 2. An image of the PCDL software used to create the compound database and MS/MS spectral library.

Figure 3. Images of PCDL software showing the MS/MS spectral data of acetyl fentanyl. MS/MS spectral data is shown at collision energies: A) $10 \mathrm{eV}, \mathrm{B}) 20$ $\mathrm{eV}$, and C) $40 \mathrm{eV}$. 50

Figure 4. Weighted calibration curve for 25I-NBF from validation Mix 2 64

Figure 5. Unweighted calibration curve for 25I-NBF from validation Mix 2. 64

Figure 6. Calibration curve for 25I-NBF in validation mix 2 from linearity studies. 65

Figure 7. Carryover study for oleamide in Mix 3 comparing analyte concentration present in a $5 \mathrm{ng} / \mathrm{mL}$ sample with that in a blank urine sample that was analyzed immediately following a $120 \mathrm{ng} / \mathrm{mL}$ sample.

Figure 8. Structures of NPS in CE mixes (see Table 21 for mixture compositions):

(1) JWH $019 \mathrm{~N}$-(2-fluorohexyl) isomer, (2) JWH $019 \mathrm{~N}$-(3-fluorohexyl) isomer,

(3) JWH $019 \mathrm{~N}-(4-f l u o r o h e x y l)$ isomer, (4) JWH $019 \mathrm{~N}$-(5-fluorohexyl) isomer,

(5) JWH 019 N-(6-fluorohexyl) isomer, (6) JWH 080, (7) JWH 203, (8) PB-22,

(9) MAM2201 N-(2-fluoropentyl) isomer, (10) XLR12, (11) JWH 073 2'naphthyl-N-(1,1-dimethylethylisomer), and (12) FUB-144.

Figure 9. Schematic of the two-position, four-port duo valve (right) used in conjunction with ta two-position, six-port valve (left) to connect the two dimensions. 100

Figure 10. Schematic for the 2D-LC setup used in final system. 101

Figure 11. Solvent gradients used in the first and second dimensions of the 2D-LC separation.

Figure 12. UV chromatograms $(\lambda=220 \mathrm{~nm})$ for $1 \mathrm{D}$ separations of (a) CE Mix 1, and (b) CE Mix 2 with the Zorbax Eclipse Plus $\mathrm{C}_{18}$ column. 106

Figure 13. UV chromatograms $(\lambda=240 \mathrm{~nm})$ for 1D separations of (a) CE Mix 1, and (b) CE Mix 2 using the Bonus-RP column over 3 min at a flow rate of 0.5 
$\mathrm{mL} / \mathrm{min}$ with a gradient of $50-95 \% \mathrm{~B}$; A: water $+0.1 \%$ TFA, and B: acetonitrile. Peak numbers correspond to compounds listed in Table 21............................... 108

Figure 14. UV chromatograms $(\lambda=240 \mathrm{~nm})$ for $1 \mathrm{D}$ separation of (a) CE Mix 1 over $5 \mathrm{~min}$, and (b) CE Mix 2 over 3 min using the biphenyl column at a flow rate of $0.5 \mathrm{~mL} / \mathrm{min}$ with a gradient of $70-95 \% \mathrm{~B}$; A: water $+0.1 \% \mathrm{TFA}$, and $\mathrm{B}$ :

$\mathrm{MeOH}$. Peak numbers correspond to compounds listed in Table 21

Figure 15. Contour plots demonstrating the 2D separation of CE mixes 1, 2, and 3. Peak number correspond to those listed in Table 21 . 


\section{LIST OF ABBREVIATIONS, ACRONYMS, AND SYMBOLS}

\begin{tabular}{|c|c|}
\hline${ }^{1} \mathrm{D}$ & First Dimension \\
\hline${ }^{2} \mathrm{D}$ & Second Dimension \\
\hline $1 \mathrm{D}$ & One-dimensional \\
\hline $2 \mathrm{D}$ & Two-dimensional \\
\hline $\mathrm{ACN}$ & Acetonitrile \\
\hline $\mathrm{AF}$ & Ammonium Formate \\
\hline APCI & Atmospheric Pressure Chemical Ionization \\
\hline CB1 & Cannabinoid Type 1 Receptor \\
\hline $\mathrm{CB} 2$ & Cannabinoid Type 2 Receptor \\
\hline $\mathrm{CV}$ & Coefficient of Variation \\
\hline DAD & Diode Array Detector \\
\hline DEA & Drug Enforcement Administration \\
\hline $\mathrm{DFC}$ & Drug Facilitated Crime \\
\hline DI & Deionized \\
\hline DUI & Driving Under the Influence \\
\hline EI & Electron Ionization \\
\hline EIC & Extracted Ion Chromatogram \\
\hline
\end{tabular}




$\begin{array}{ll}\text { ESI } & \text { Electrospray Ionization } \\ \text { FA } & \text { Formic Acid } \\ \text { FBF } & \text { Find by Formula } \\ \text { GC } & \text { Gas Chromatography } \\ \mathrm{H}_{2} \mathrm{O} & \text { Water } \\ \text { HILIC } & \text { Hydrophilic Interaction Liquid Chromatography } \\ \text { HPLC } & \text { High Performance Liquid Chromatography } \\ \text { HRMS } & \text { High Resolution Mass Spectrometry } \\ \text { IMS } & \text { Ion Mobility Spectrometry } \\ \text { mL } & \text { Internal Standard } \\ \text { me } & \text { Milliliter } \\ \text { LC } & \text { Liquid Chromatography } \\ \text { LOD } & \text { Limit of Detection } \\ \text { LOQ } & \text { Limit of Quantitation } \\ & \text { Methylenedioxy Pyrovalerone } \\ \text { MDP } & \\ \text { Metrix Effect }\end{array}$




\begin{tabular}{|c|c|}
\hline MRM & Multiple Reaction Monitoring \\
\hline MS & Mass Spectrometry \\
\hline MS/MS & Tandem Mass Spectrometry \\
\hline$m / z$ & Mass to Charge Ratio \\
\hline ng & Nanogram \\
\hline NP & Normal Phase \\
\hline NPS & Novel Psychoactive Substances \\
\hline OSAC & Organization of Scientific Area Committees \\
\hline PCDL & Personal Compound Database Library \\
\hline PCP & Phencyclidine \\
\hline PDA & Photodiode Array \\
\hline ppm & Parts Per Million \\
\hline QC & Quality Control \\
\hline QQQ & Triple Quadrupole Mass Spectrometer \\
\hline QTOF & Quadrupole Time-of-Flight Mass Spectrometer \\
\hline $\mathrm{RP}$ & Reversed-phase \\
\hline RSD & Relative Standard Deviation \\
\hline SAMHSA & Substance Abuse and Mental Health Services Administration \\
\hline
\end{tabular}




$\begin{array}{ll}\text { SEC } & \text { Size-exclusion Chromatography } \\ \text { SWGTOX } & \text { Scientific Working Group for Forensic Toxicology } \\ \text { TIC } & \text { Total Ion Chromatogram } \\ \text { TFA } & \text { Trifluoroacetic Acid } \\ \text { UV } & \text { Ultraviolet } \\ \mu \mathrm{g} & \text { Microgram } \\ \mu \mathrm{L} & \text { Microliter }\end{array}$




\section{INTRODUCTION}

\subsection{Statement of the Problem}

The emergence of many novel psychoactive substances (NPS) has caused great concern in the areas of public health and law enforcement. NPS include a wide variety of diverse compounds such as synthetic cannabinoids, designer opioids, designer hallucinogens, and synthetic stimulants for which multiple reports of overdoses and even fatalities are available. ${ }^{1-4}$ These compounds are mainly created with the purpose of circumventing existing drug laws. Manufacturers achieve this goal by simply altering the existing structures of NPS and other drugs (i.e., adding or removing a functional group, moving a functional group, etc.). When changes are made to the structures, the new compounds no longer match the illegal structure and therefore do not fall under the regulation of controlled substance laws. ${ }^{5}$ As a result, there exist thousands of these compounds whose effects can range from having no pharmacological effect to exhibiting significant toxicity.

Changing the chemical structures of these compounds can also make existing methods of detection unreliable, since these methods are generally designed to identify specific functional groups or structures. ${ }^{6}$ In the fields of clinical and forensic toxicology, the changing structures can especially cause issues when trying to identify which compound was used in instances of overdose and emergency department cases in order to properly treat patients. To combat the public health issue of NPS, a reliable method for detection and identification of the multitude of NPS potentially present in biological specimens must be available. 
One solution for detecting and identifying NPS that might be missed by other screening methods involves the creation of drug libraries for chromatographic and mass spectrometric data. These libraries contain characteristic spectral information generated using mass spectrometry (MS) that can help identify NPS in specimens. Currently, there exist a number of libraries for gas chromatography (GC) generated via electron ionization (EI) methods, but fewer for liquid chromatography (LC) and electrospray ionization (ESI) methods. For example, Ojanpera, Broecker, and Rosano have all created libraries for LC, but they are either mostly theoretical or do not contain many NPS which can hinder identification efforts during screening and confirmation. ${ }^{7-9}$

The lack of suitable screening methods and support libraries has hindered the ability of forensic and clinical laboratories to quickly and reliably identify NPS. Typically, these compounds are not detected and identified until there are incidences of mass-intoxications or a series of deaths in a singular region. To rapidly identify such incidents as they occur, laboratories need to have access to constantly updated mass spectral databases that consist of accurate precursor mass and fragmentation data. ${ }^{10}$

\subsection{Rationale for Research}

In most toxicology laboratories, drug screening typically involves the use of immunoassays. While these methods are fairly effective in screening for common drugs

of abuse, they are not well suited for the screening of specimens containing NPS. ${ }^{6,10-12}$ A major goal of the present work was to develop a reference standard-based spectral library containing high resolution mass spectrometry (HRMS) data for more than 800 NPS and related compounds for use with an LC-quadrupole time-of-flight (QTOF)-MS based screening method. The developed library will help enable the rapid detection and 
identification of NPS in biological specimens such as urine. The library can also be used with retrospective data searching in order to detect and identify previously unreported NPS in specimens.

Following validation of the LC-QTOF-MS method, blind-spiked urine specimens were screened in order to establish the applicability of the method for use with real-world specimens. The specimens used for this part of the research were created in a "blind" manner, meaning that they were designed and made by a third party directly involved with the analysis.

It was also important to develop a comprehensive screening and confirmation method capable of detecting and identifying several hundred NPS in a single analytical run with high specificity at minimal (i.e. low $\mathrm{ng} / \mathrm{mL}$ ) concentrations. In order to accomplish this goal, a rapid, sensitive, and specific LC-QTOF-MS based analytical method was developed and validated in conjunction with the compound database and HRMS libraries. In some cases, however, it can be extremely difficult to chromatographically separate certain NPS to facilitate identification via mass spectrometry, such as in the case of chemically similar and isobaric compounds which have the same or significantly related chemical formulae. This lack of separation can be especially problematic when attempting to identify a previously unknown or unreported NPS using its mass spectra alone. To address the challenge presented by lack of separation, a two-dimensional (2D)-LC separation system was investigated to separate isobaric and non-isobaric co-eluting SC. 


\subsection{Significance of Study}

The body of work presented herein has applicability to forensic science, toxicology, law enforcement, and even pharmaceutical development. The research provides a large HRMS library for the identification of over 800 common and uncommon NPS, metabolites, and related compounds, generated using ESI. A validated, comprehensive LC-QTOF-MS method for screening and confirmation was also developed in conjunction with the library and tested using blind-spiked urine specimens to ensure applicability. A possible solution to the separation challenge presented by the co-elution of some NPS, both isobaric and non-isobaric, was also investigated through 2D-LC analysis of mixes containing co-eluting synthetic cannabinoids (SC).

The NPS used in the research were identified and selected on the basis of the reference standards available from commercial suppliers, as well as citations in government documents, peer-reviewed literature, and online drug-user forums. In order to complete this research, the work was divided into three major tasks.

\subsubsection{Task 1 - Development of database and spectral library}

Comprehensive libraries are widely used in analytical toxicology for the identification of analytes present in specimens, however, these libraries often do not contain many NPS. In order to identify NPS, a database and library containing characteristic spectra of these compounds must be developed. This report details the creation of a database containing over 800 NPS, metabolites, and related compounds, as well as the generation and collection of tandem mass spectrometry (MS/MS) data for each compound using commercially available reference standards. The database and 
library were then used to qualitatively screen blind-spiked urine specimens to ensure applicability to real-world samples.

\subsubsection{Task 2 - Comprehensive LC-QTOF-MS method validation}

In order to detect and identify NPS and related compounds in biological specimens, a comprehensive LC-QTOF-MS method for screening and confirmation of said compounds was developed and validated according to accepted analytical method development guidelines.

\subsubsection{Task 3 - Investigation of a 2D-LC method}

Chemically related and isobaric NPS are not uncommon and can present challenges to identification using mass spectral data alone. Throughout the development

of the library and LC-QTOF-MS method, several such compounds were identified which demonstrated similar chromatographic retention times by standard 1D-LC.

Consequently, 2D-LC was investigated as a possible approach for separation of coeluting, chemically related and isobaric SC in order to analyze each compound individually. 


\section{BACKGROUND}

\subsection{Novel Psychoactive Substances}

Novel psychoactive substances (NPS), also known as "designer drugs", are compounds that have been created to circumvent controlled substance laws. When the Comprehensive Drug Abuse Prevention and Act, also known as the Controlled Substances Act (CSA), was passed into legislation in 1970, it created a protocol for regulating substances depending on their potential for abuse, potential for addiction, and accepted medical usage. Compounds that had high potentials for abuse and addiction, but with no or limited accepted medial use were classified as Schedule I or II substances, respectively. ${ }^{13}$

Typically, NPS have been synthesized to be structurally or pharmacologically comparable to substances that have been identified as Schedule I or II drugs by the U.S. Drug Enforcement Administration (DEA). ${ }^{5,10}$ In the late 1980s, an addition to the CSA, known as the Federal Analogue Act, was made to allow for the scheduling of NPS that were structurally and pharmacologically "substantially similar" to Schedule I or II substances under DEA control. ${ }^{14,15}$ In order to get around these legal controls, illicit laboratories turn to a variety of sources to guide the synthesis of new compounds that are not yet under government control. These sources may include scientific journal articles, patents, and books published by pharmaceutical companies, academic or research institutions, and other organizations as part of the legitimate scientific process. ${ }^{15}$

For classification purposes, NPS are generally divided according to their chemical structure. These classes include, but are not limited to, piperazines, phenethylamines, tryptamines, cathinones, opioids, and synthetic cannabinoids. ${ }^{16,17}$ With the rise of 
internet access over the past two decades, not only has synthesis information become more widely available, but the ability to market and traffic these compounds has resulted in an explosion of new, untested NPS on the market. In addition to the issues with legality, NPS also represent a significant risk to public health, given the untested nature of these compounds and the potential for adverse effects associated with their usage as compared with more traditional drugs of abuse. ${ }^{5,17,18}$

One example involves the emergence of illicit fentanyl and its derivatives as part of the on-going opioid crisis in the United States. Fentanyl is a synthetic opioid that is approximately 100 times as potent as morphine. When the analog $\alpha$-methylfentanyl was classified as a Schedule I drug by the DEA in 1981, it took just a few years for another analog, $\alpha$-acetylfentanyl, to appear in 1984, demonstrating the efficiency with which illicit drug manufacturers have historically been able to introduce new compounds. ${ }^{5}$ New fentanyl derivatives now appear on the street in a time frame of weeks to months rather than years.

More recently, numerous synthetic cannabinoids have been scheduled, only to have analogues appear on the market shortly thereafter. The synthetic cannabinoid JWH018 was sold as a component of herbal incense products such as 'Spice' and 'K2,' and was labeled "not for human consumption" in order to avoid regulation. However, JWH018 exhibits effects at a higher potency than $\Delta^{9}$-tetrahydrocannabinol (THC), which is the psychoactive component in cannabis. ${ }^{19}$ As a consequence of these effects, the DEA temporarily placed JWH-018 on the Schedule I list in 2011. That very same year, NutraGenomics, a company that sold synthetic cannabinoids, stopped selling JWH-018 products. Instead, they began selling analogs of JWH-018 such as AM-2201, whose 
structure differs only by the substitution of a fluorine for a hydrogen at the end of the alkyl chain. It was not until 2012 that AM-2201 was controlled as a Schedule 1 substance. $^{20,21}$

Synthetic cannabinoids are not the only class of NPS that have been subjected to fast turn-around times by illicit manufacturers. The phenethylamine compound 4-methyl-Nmethylcathinone, also known as mephedrone, was placed on the temporary Schedule 1 list in October 2011 because it can cause seizures, increase blood pressure and heart rate, result in delusions, and even cause death. ${ }^{22}$ After permanent scheduling occurred in July 2012, the compound 4-fluoro-N-methylcathinone (4-FMC) appeared exhibiting similar effects after substitution of a fluorine for the para-position methyl group of the benzene ring. This compound was not placed on a schedule by the DEA until March 2014. ${ }^{23}$

\subsection{Analysis of Drugs in Biological Specimens}

The analysis of drugs of abuse, including NPS, in biological specimens represents a large challenge for toxicologists. Methods for such analyses need to take into account not only the analytes of interest, but also any potential interferences or sample preparation issues that arise as a result of biological matrices and any endogenous or naturally occurring compounds within the specimen.

The most common matrices encountered in forensic toxicological analyses include blood and urine. ${ }^{6,24}$ Drug concentrations in both matrices can provide valuable information about a person's exposure to a drug, including the identity of the compound and, in some cases, an approximate time since exposure. Analysis of urine also presents the option of detecting and identifying metabolites, providing another option for targeted analysis in cases when the parent compound cannot be detected using current methods. 
For example, synthetic cannabinoid metabolites are the most prevalent in urine with minimal or even no parent compound present. The presence of any one of these metabolites can then indicate exposure to the parent drug. ${ }^{25}$

Both urine and blood are fairly easy to obtain, however, urine collection is much less invasive and is thus a very common specimen collected for analyses, especially those for antemortem toxicology. Larger volumes of urine than blood may also be collected at any one time, providing a significant advantage in the ability for multiple analyses and re-analyses to be conducted on a single specimen. ${ }^{8,26}$ These benefits make urine a good option for screening in clinical and forensic toxicology, including cases such as compliance monitoring, workplace drug testing, drug rehabilitation, child welfare, doping control, drug-facilitated crime (DFC), driving under the influence (DUI), and more. ${ }^{27}$ In clinical and forensic toxicological analyses, urine is an important matrix for several other reasons. A major advantage of conducting analyses with urine is that there is a much longer window of detection for drug compounds in the matrix. This window can extend to several days after exposure, as opposed to blood concentrations which typically dissipate after a few hours. Both parent drug and metabolites can often be detected in urine providing more targets to analyze to indicate extent and timing of exposure.

Another key advantage to using urine is that drug compounds and their metabolites tend to be more concentrated. As substances are metabolized, they accumulate in the bladder and when excreted in urine produce a specimen in which the analytes of interest are present at higher concentrations, even if the user was exposed to only a low dose of the compound. ${ }^{28}$ Concentrated samples present a huge benefit for 
qualitative analysis, however, quantitation for the purposes of correlation to levels of intoxication is much more difficult as compared to blood specimens.

From a sample preparation standpoint, urine is much easier to work with than blood since many analyses can be conducted simply by diluting the urine specimen with water, as opposed to the sometimes laborious extraction procedures required for the analysis of blood (i.e., solid-phase extraction). Liquid-liquid extraction (LLE), which involves several steps in order to selectively remove acidic and basic compounds, can also be applied to urine, but for purposes of simplicity, dilution of the specimen is a valid technique for urinalysis. ${ }^{29-32}$

Consequently, while there are some drawbacks to urine analysis, including the inability to correlate drug concentration with levels of intoxication, urine is a very valuable matrix for the qualitative analysis of small molecules and drug substances in both clinical and forensic toxicology settings. ${ }^{33}$

Presumptive screening tests are the first step in the detection and identification of analytes of interest in biological specimens. These screening methods are needed for fast delineation of negative samples from positive ones, and to also generate preliminary information as to what analytes might be present in any positive specimens. ${ }^{34}$ Screening techniques look for selected analytes present at concentrations above a specific level known as the "cutoff" concentration. A predetermined set of analytes to be searched, or screened for, in a single run is known as a drug "panel". One of the most commonly used panels for drug screening, particularly in workplace drug monitoring, is known as the Substance Abuse and Mental Health Services Administration (SAMHSA) Five. This panel includes tests for five common categories of drugs: amphetamines, cocaine, 
marijuana, opiates, and phencyclidine (PCP). Other categories that might be included are barbiturates, benzodiazepines, hydrocodone, methylenedioxy-methamphetamine (MDMA), and methadone.$^{35}$ However, screening tests typically include few, if any, NPS. The need for accurate screening methods cannot be understated, especially since many current screening methods are not reliable for use in detecting NPS; oftentimes generating a negative result when NPS are actually present. ${ }^{36,37}$

The type of screening method used depends on the matrix being analyzed, but typical methods for urine specimens include immunoassays to determine which class of drugs or NPS may be present, as well as liquid chromatographic (LC) and gas chromatographic (GC) methods coupled with mass spectrometry (MS). Screens that result in positive results then undergo confirmatory testing in order to accurately identify the compound(s) present and provide quantitation when required. However, this process of screening and confirmation can only be followed if compounds are known and if the immunoassay is capable of detecting NPS. ${ }^{36,38}$

Immunoassays are a type of immunochemical technique which can provide a simple answer to whether or not a specific drug or drug class is present above cut-off concentrations. These assays represent a relatively quick, inexpensive, and user-friendly method to determine which drug or class of drug is present, if any at all. ${ }^{12,39,40}$ When a compound is detected and tentatively identified using the results of the immunoassay screen, a more selective confirmatory technique, such as mass spectrometry, is then used for specific identification. However, immunoassays use antibody-antigen interactions with the analytes of interest serving as the antigen. The antibodies employed by the assays are engineered to detect specific chemical structures characteristic of one or more 
drug classes. As a result, the slight structural alterations present in NPS can be enough to cause a negative result, or no cross-reactivity, regardless of whether the NPS has been previously identified or not. ${ }^{12,41}$ In these instances, specimens are determined to be "negative" for drugs and are not submitted for further analyses. ${ }^{39}$ For example, mephedrone and methylenedioxy pyrovalerone (MDPV) are two of the most common illicit stimulant-type substances. When immunoassays were used to try and screen for these compounds, there was little to no cross-reactivity demonstrated, meaning that these samples would be determined to be negative and likely discarded. ${ }^{41}$

A published work by Swortwood et al. sought to determine the level of crossreactivity present when 16 commercial immunoassay kits were used to screen for 30 NPS from the phenethylamine, piperazine, and tryptamine classes of compounds in human serum. The kits chosen included some designed to detect amphetamine and/or methamphetamine-like compounds, as well as a few more specific kits such as one solely for the detection of mephedrone and methcathinone. Ultimately, the commercial kits demonstrated little to no cross-reactivity with the NPS chosen for evaluation. Those that demonstrated minimal cross-reactivity did so at concentrations too high to be practical for forensic or clinical applications. ${ }^{6}$ Results from such studies have shown that immunoassays, as they currently exist, cannot be reliably used as a presumptive detection method for NPS within the same drug class, let alone comprehensively. While some newer immunoassay kits have been designed for the presumptive identification of a few NPS classes, specific immunoassays are not widely available for many NPS or their derivatives. 
As the structures of NPS are constantly changing, it is not feasible to create the specific antibodies needed for immunoassays rapidly enough to keep pace with introduction of new varieties of NPS. In addition, existing kits cannot be expected to demonstrate cross-reactivity with newer NPS as they emerge on the market. Therefore, different methods must be employed for comprehensive screening of NPS in biological specimens to account for the lack of reliable detection with immunoassay-type methods.

\subsection{Liquid Chromatography Mass Spectrometry}

In order to overcome some of the limitations of screening with immunoassays, and to increase specificity, methods involving GC or LC coupled to MS have been used for both targeted and non-targeted screening purposes of analytes in biological specimens. Targeted screening occurs when the presence of a specific known compound or set of compounds is searched for in the sample matrix and only MS data associated with the selected compound(s) are collected. Non-targeted analyses involves the use of broad screening methods in which all MS data are collected and then analyzed afterwards to identify any compounds that might be present. Non-targeted methods are ideal for unknown screening, since analytes that might be of interest are not unintentionally excluded during data collection as they might be in targeted methods. ${ }^{28,40}$

Both targeted and non-targeted methods involve the chromatographic separation of compounds from the specimen matrix, as well as from other compounds that may be present in a mixture, before detection by MS. Within the mass spectrometer, analytes are ionized and can be detected as the original molecule, or can be subjected to an energy source and broken into fragments. These fragmentation patterns are characteristic of the 
original molecule and can then be used for the structural elucidation and identification of analytes present. $^{42-45}$

When using MS-based techniques in conjunction with either GC or LC separation to identify an analyte, mass spectral libraries are required. Typically, ion fragmentation patterns are generated via GC- or LC-MS and these patterns are then compared with those present in existing libraries which can contain information for several thousand different compounds. ${ }^{42,46,47}$

Gas chromatography with mass spectrometry (GC-MS) has been described as the "gold standard" for drug screening and identification. As an established and wellunderstood technique, GC-MS is used by many labs for the detection and identification of NPS and many other compounds in both toxicological and seized material analyses. Large spectral libraries containing tens of thousands of spectra have been built over the course of 40+ years using electron ionization (EI) techniques; a hard ionization technique which typically produces fragment-rich, characteristic spectra. ${ }^{42,48}$ In the 2017 iteration of the EI mass spectral library released by the National Institute of Standards and Technology (NIST), over 260,000 unique compounds are represented by over 300,000 EI spectra. $^{49}$

While there exist large databases of reproducible spectral data generated through the use of EI sources, the use of GC-MS is not without its restrictions, particularly in the types of analytes that can be evaluated with this technique. In order to generate spectra using EI sources, compounds of interest must be volatile, non-polar, and thermally stable. Lengthy sample preparation processes such as derivatization are sometimes required to make some compounds suitable for analysis using EI, while the above suitability criteria 
for analysis might prevent some analytes from being detected at all. ${ }^{50}$ The lengthy sample preparation restriction is especially true for the analysis of urine specimens that require cleavage of the glucuronic acid or sulfonate acid conjugates of the phase I metabolites that may be present during analysis via GC. ${ }^{8}$

In contrast, liquid chromatography with mass spectrometry (LC-MS) is suitable for the analysis of non-volatile, polar, and thermally labile compounds and does not mandate that specimens undergo derivatization or other chemical modifications prior to analysis. ${ }^{8,36,38,42,47,51}$ Electrospray ionization (ESI), commonly used in LC-MS, is a soft ionization technique that is effective in ionizing analytes contained in aqueous specimens without requiring the derivatization often needed in GC-MS. As a result, LC-MS screening has increased in popularity for clinical and forensic toxicological case work because of the increasing polarity and low volatility of many new relevant substances which are difficult, if not impossible, to analyze via GC-MS.

\subsubsection{Electrospray Ionization Spectral Libraries}

Since the use of LC-MS for screening is much newer than the use of GC-MS, the spectral libraries that have been created are not as extensive. The very nature of the ionization techniques used with LC-MS are also not as reproducible as those used with GC-MS, and require complete standardization of source parameters in order for libraries to be used across different laboratories and instruments. ${ }^{42,50,52}$ Additional work is required in the development of large, comprehensive mass spectral libraries to support the identification of compounds using LC-MS methods and their corresponding ionization techniques, particularly electrospray ionization (ESI). ${ }^{25,28,45,53}$ 
Techniques using LC-MS for identifying and quantifying NPS require the use of libraries with known compounds and their known masses, spectral data, or ion transitions. ${ }^{18,54}$ If a NPS has not been previously included in such a database or library then detection may be possible with a non-targeted analytical method, however, identification and thus quantification will not be achievable. ${ }^{10,18}$ Lack of analyte detection due to absence in a database or library is also true for NPS metabolites, which are of particular interest in forensic toxicological analyses, especially those from the synthetic cannabinoid classes which can be abused in the same manner as the parent compound..$^{55}$

Electrospray ionization (ESI) is a soft ionization technique commonly used with LC-MS to generate ions detectable by the MS detectors. Libraries created using ESI coupled to tandem mass spectrometry (ESI-MS/MS) have been shown to be much more reproducible than those created with "in-source" collision induced dissociation (CID), such as with methods utilizing a single mass analyzer, so long as collision energy and collision gas pressure are consistent. ${ }^{47,53,56,57}$ This is because the first mass analyzer can be used as a filter to isolate a particular range of masses and exclude ions from background contaminants and matrix components before CID fragmentation occurs. Selective ion fragmentation then occurs in the collision cell placed after the first mass analyzer. The resultant product ions then move through the second mass analyzer where they are analyzed using mass-to-charge $(\mathrm{m} / \mathrm{z})$ ratios. ${ }^{53,56,58}$ The first mass analyzer can also be used to detect analytes before undergoing a mass dependent scan which provides the MS/MS spectra needed for identification via a library search. ${ }^{38}$ The use of ESIMS/MS has the added benefit of virtually always revealing the molecular ion (i.e., the 
ionized original compound) in resultant spectral data, leading to increased confidence in identification from library searching. ${ }^{42,54}$

Libraries for ESI-MS/MS techniques are typically generated using triple quadrupole $(\mathrm{QqQ})$ or hybrid quadrupole time-of-flight (QTOF) mass analyzers with fragmentation patterns collected at more than one collision energy to account for differences between mass analyzers and brands. ${ }^{42,47,53,58}$ Most ESI libraries exist inhouse, but there have been published works creating reproducible and shareable libraries. . $^{8,9,28,34,36,38,50,57,59,60}$

The poor availability of reference standards for many NPS and metabolites presents a large challenge to forensic toxicology laboratories when trying to detect and identify both known and unknown NPS and other xenobiotics. In an attempt to address this issue, the Ojanperä group created a database containing theoretical monoisotopic masses for over 7,500 toxicologically relevant compounds and metabolites. Their database was then used in conjunction with a method for LC-TOF-MS and applied to postmortem human urine specimens. Each search of the database resulted in no more than three potential elemental formulas which resulted in a significantly more manageable list of possible identifications. The greatest asset to this database was that it could be updated with new formulas and theoretical masses as soon as they appear in the literature or are noted by law enforcement and/or public health officials. ${ }^{27}$ While this approach simplified the list of possible identification significantly, the use of accurate mass data alone was not enough to explicitly identify the compounds present. Retention data could have been used to corroborate identifications, but reference standards would have been needed in order to acquire those data. ${ }^{8}$ 
Another approach to theoretical databases was employed by Polettini et al. when they developed a screening procedure utilizing a subset of compounds curated from those available in PubChem. The PubChem database contained accurate masses for over 50,000 toxicologically relevant compounds including pharmaceutical and illicit drugs, pesticides, poisons, and over 6,000 metabolites. The database was then used to screen for compounds present in a variety of postmortem biological specimens. The resulting number of possible identifications from this work ranged from one to 39 per analyte. ${ }^{60}$ While Polettini's work indicated the potential of a theoretical database to help narrow down possible identifications, unambiguous identifications could not be generated for each analyte. Other information that would assist in improving the confidence of an identification include chromatographic retention data, isotope patterns, and/or fragmentation patterns. ${ }^{60,61}$

Another possible way to address the issue of the lack of available reference standards, especially of NPS metabolites, involves the use of in silico methods to predict metabolites of certain compounds and what their characteristic fragmentation patterns might be. For example, Pelander et al. used metabolite prediction software to predict the phase I metabolites of the anti-psychotic drug quetiapine. Using another software tool, fragmentation patterns of these metabolites were also predicted. The resulting data were used to screen authentic urine specimens in which several of the predicted major metabolites were detected, however, there were several metabolites detected that had not been predicted, particularly the hydroxylated metabolites. The predicted fragmentation patterns were also helpful in differentiating between isobaric metabolites, but the work was not conclusive enough to serve as a reliable substitute for identification using CID 
spectral profiles collected from reference standards. ${ }^{62}$ Predicted fragmentation patterns would not be suitable for use in forensic cases as they would not meet standards for admissibility in court.

In a recent paper by Colby et al., the efficiency of using certain workflows for screening via LC-QTOF-MS was evaluated when using databases and/or spectral libraries to identify compounds of interest. Four different workflows were assessed in the identification of 170 drugs and metabolites: one targeted and three involving "suspect screening." The first involved analysis of a reference standard followed by targeted searching of the sample. In contrast, suspect screening does not utilize a reference standard but instead bases identification on predicted and/or intrinsic characteristics such as accurate mass, isotope pattern, and product ion spectrum. The study focused on which combination of these three characteristics carried the most weight when identifying compounds without direct use of a reference standard. It was found, unsurprisingly, that the most effective methods included the use of product ion spectra that had been previously collected from reference standards and included in a searchable library. When product ion spectra were utilized, in addition to accurate mass and isotope patterns, over $80 \%$ of the drugs in human urine specimens were correctly identified with a minimal number of false identifications. These results, combined with the fact that retention times were not required, indicated the potential of building large screening methods and libraries for screening of toxicologically relevant compounds, including NPS. ${ }^{63}$

The utility of spectral libraries for identification of compounds in screening procedures has been demonstrated in other works as well. Lee et al. created a screening method for toxicologically relevant compounds present in urine which utilized a spectral 
library developed with the use of reference standards. Spectral data were collected using ultra performance liquid chromatography (UPLC) coupled to a TOF MS then searched against a library containing spectra for 300 compounds, 102 of which were metabolites. The substances in the library originated from pure reference standards, pharmaceutical materials, and from metabolites present in authentic urine specimens. The library was created using retention data, exact mass, and fragmentation patterns collected at two collision energies ( 10 and $45 \mathrm{eV}$ ) for each of the compounds. The inclusion of spectra containing a pseudomolecular ion in addition to one CID fragmentation pattern provided additional confidence in the identification of the compounds present in the samples and improved the specificity of the method. ${ }^{50}$

The largest MS library, containing CID mass spectral data for over 2,500 toxicologically relevant substances, was created by Broecker et al. A hybrid quadrupole time-of-flight mass spectrometer (QTOF-MS) was used to collect the CID fragmentation patterns generated in the collision cell located between the quadrupole and the TOF analyzer. Substances were subjected to three different collision energies (10, 20, and 40 $\mathrm{eV}$ ) and the data were then curated and checked for suitability before being added to an existing database of theoretical fragment masses and molecular formulas for 7,500+ additional toxicologically relevant substances. The compounds in the combined spectral library and database represented substances such as illicit and therapeutic drugs, pesticides, alkaloids, and other toxic chemicals and metabolites. ${ }^{7}$ However, there was not a significant presence of NPS and NPS metabolites as is true for many other existing ESIMS/MS libraries and databases. This lack of NPS representation in existing libraries can hinder the detection and identification of such substances in systematic clinical and 
forensic toxicological analyses. Thus the development of larger, more comprehensive libraries that include NPS is needed to improve detection and identification of compounds during screening efforts.

Historically, QqQ-MS, a unit resolution technique, is more often used for drug screening than HRMS because of its lower cost of operation and its robustness. However, since the mass spectra generated are of low resolution, compounds must be known prior to analysis. Libraries for QqQ-MS are built by collecting data generated from multiple reaction monitoring (MRM), which is suitable for use with targeted screening methods. ${ }^{7,37,54}$ High resolution MS methods, such as those utilizing QTOF-MS are recognized as having higher resolution and allowing for the collection of all ion spectra, an approach that is ideal for non-targeted screening. High-resolution mass spectra can also be collected for the screening of known targets using data-dependent acquisition methods. ${ }^{7,36,40,64}$ Compounds are then identified using corresponding libraries through the comparison of accurate masses or the characteristic spectral data. ${ }^{8,65}$ Methods using QTOF mass analyzers are recognized as having high mass accuracy and high-resolution capabilities, meaning that collected data have mass accuracy better than $5 \mathrm{ppm}$ and resolution greater than 20,000 full width half maximum (FWHM), respectively. However, high mass accuracy is not required for a method to be considered high-resolution. ${ }^{28}$ These qualities are vital in building HRMS libraries to ensure the collection of accurate masses, as well as the ability to resolve isobaric compounds or those with very similar chemical formulas and similar product ion fragmentation patterns. An added benefit of using QTOF mass analyzers is that the accurate masses of analytes can be recorded over a wide range of abundances. The 
ability to record over a wide abundance range is especially important for conducting both targeted and non-targeted analyses, which enables scientists to conduct screening for known analytes and also to collect ion data that can be retroactively searched for previously unknown substances as libraries are updated. ${ }^{18,28,66}$ The ability to conduct non-targeted analyses and collect data that can be searched later on are crucial to the future of forensic toxicological analysis of NPS, as the potential to create new drug compounds within synthetic chemistry is practically unlimited. ${ }^{67,68}$

Employing QTOF mass analyzers also enables users to search for common fragments or use mass-defect filtering to investigate unknown compounds that share common functional groups or structural components with known NPS. ${ }^{69}$ When such collected spectra are searched against a library, a "hit list" of possible compound identities is generated. These lists are comprised of "scores" which reflect the likelihood that the collected spectra and the library spectra are from the same compound. ${ }^{42,43,47}$ In instances where the collected spectrum is from a compound not yet included in the library, modern algorithms will include library compounds on the "hit list" that may differ by a simple insertion, deletion, or replacement of a structural group. These "nearest neighbor" identifications are extremely useful when screening unknown NPS and are much more impactful when using HRMS spectra. ${ }^{44}$

There are two main search methods used when comparing collected spectra and library spectra: forward and reverse searching. Forward searching involves comparing the collected spectrum of an unknown with spectra contained in a library. The base mass peak or each ion within the spectrum is identified and then compared to those contained in the library for potential matches. Reverse searching is essentially the same technique 
except that the collected spectrum is searched through using spectra in the library. More simply, in forward searching, the collected spectrum is searched for in the library spectra and in reverse searching library spectra are searched for in the collected spectrum. ${ }^{7,48}$

\subsection{Co-elution Challenges}

Even with the increased resolving power of HRMS, there may still be instances in which isobaric compounds or those that are too structurally related to be identified solely by mass spectral data are present in specimens. This is particularly true with NPS as many are simple variations on existing compounds with minor or novel structural alterations. In cases of mixtures or true unknown substances, resolution of compounds using spectral data alone may not be possible.

Typically, chromatographic separation systems are employed to isolate individual compounds prior to MS analysis. However, there are instances in which the structural or physiochemical differences between NPS are so slight that they cannot be separated using traditional chromatographic techniques (i.e. LC or GC) and will therefore not be detected as individual compounds. ${ }^{70-72}$ To resolve this issue, two-dimensional liquid chromatography (2D-LC) has been proposed as a method to improve separation and resolution of complex mixtures prior to further mass spectral analysis. ${ }^{73,74}$

\subsubsection{D-LC}

When conducting toxicological analyses that rely on searching MS library data, screening for unknown or previously unreported NPS can be problematic because of the large number of isomers and chemically related compounds that exist with similar accurate masses, fragmentation patterns, and/or chromatographic retention times. The alteration of a single functional group may result in the inability to separate such 
compounds using traditional chromatographic methods which can further hinder proper detection and identification of each as an individual substance. As more and more NPS are added to an analytical method in order to keep up with the growing number of possible analytes and the increasing complexity of mixtures, more separation issues are likely to arise. These challenges to separation and thus identification will only increase as more NPS are synthesized and introduced to the illicit market. ${ }^{12,70,72,75,76}$ In these instances, it is important to perform an effective initial separation so that each substance may be analyzed via MS individually. ${ }^{45,77}$ One proposed solution to issues of coelution is the use of two-dimensional liquid chromatography (2D-LC).

Two-dimensional LC combines the separation and resolving power of two independent, orthogonal LC dimensions in order to improve chromatographic separation of analytes from matrices and/or each other and to increase the maximum number of peaks that can be equally resolved in a separation space, also known as peak capacity. ${ }^{78}$ The two dimensions can consist of several columns, but typically each dimension contains a single column with different selectivity, or orthogonality, than the other. ${ }^{71,79,80}$ There have been various applications of 2D-LC ranging from analysis of proteins and peptides, determination of pesticides, separation of chiral compounds, and the separation of pharmaceuticals and other small molecules. Several of these applications also include using the improved separation and resolving powers to differentiate analytes of interest from toxicologically relevant biological matrices such as urine, whole blood, serum, and saliva. ${ }^{72,76,78,79,81-84}$

The ability of a chromatographic system to separate constituents of a mixture is commonly described by its peak capacity, which is a measure of the theoretical maximum 
number of peaks that can be equally resolved within a separation space. Traditional 1DLC systems generally achieve peak capacities of only a few hundred, whereas 2D-LC can achieve maximums over 1000. As peak capacity increases, so too does the resolution of the separation system. ${ }^{78,85,86}$

The most basic model for calculating peak capacity is for the "comprehensive" 2D-LC approach (discussed below), where the peak capacities of each dimension are multiplied as demonstrated in Equation 1, where $n_{c, t o t}$ is the theoretical peak capacity of the 2D-LC system, and ${ }^{1} n_{c}$ and ${ }^{2} n_{c}$ represent the peak capacities of the first and second dimensions, respectively. ${ }^{87}$

$$
n_{c, t o t} \approx{ }^{1} n_{c} x^{2} n_{c}
$$

However, there is no general consensus as to how peak capacity can be best calculated, since the ideal capacity values are never fully realized as a consequence of the unique designs and applications of each 2D-LC system. ${ }^{78,86}$ In contrast, "heart-cutting" 2D-LC techniques (discussed below) do not require peak capacity calculations since the only relevant chromatographic fraction is the targeted one, and thus calculations of peak capacity, which are employed to determine the maximum number of peaks that can be resolved in a separation window, hold no significant value. ${ }^{79}$

In addition to the increased power of separation, one of the greatest advantages of 2D-LC is the decreased amount of time needed for sample preparation. The potential loss of analytes during preparation can also be minimized by using 2D-LC techniques in which the first dimension is used to separate analytes of interest from complex matrices, 
as well as from other analytes present which can also be particularly useful when removing endogenous compounds from the biological matrices relevant to toxicological analyses. ${ }^{88-91}$ Using a dimension in which both cleanup and initial separation can be conducted also minimizes the amount of time required for sample preparation prior to analysis..$^{92}$

There are two main modes of operation for 2D-LC: heart-cutting (LC-LC) and comprehensive (LC x LC). Heart-cutting is a method which involves taking selected fractions or peaks of the effluent from the first dimension $\left({ }^{1} \mathrm{D}\right)$ and subjecting these to additional separation in the second dimension $\left({ }^{2} \mathrm{D}\right)$ with the remaining effluent going to waste. Heart-cutting is useful for conducting targeted analyses of analytes in complex matrices and biological specimens, since the known analytes or peaks eluting from ${ }^{1} \mathrm{D}$ are the only fractions subjected to further separation by ${ }^{2} \mathrm{D} .{ }^{93-96}$

Heart-cutting methods are commonly used in pharmaceutical laboratories to help separate target compounds and any possible impurities of a developed drug compound. Sandra et al. used a multiple heart-cutting (mLC-LC) system for the characterization of antibody-drug conjugates (ADCs) consisting of monoclonal antibodies, cytotoxic small molecule drugs, and linkers which are used in the treatment of tumor cells. ${ }^{94,97}$ The mLC-LC configuration involved using multiple sample loops, or parking decks, between the dimensions where up to 12 fractions from ${ }^{1} \mathrm{D}$ could be stored before transfer to ${ }^{2} \mathrm{D}$ for analysis. Multiple heart-cutting was a very effective method, but the fractions that were selected to go to the "parking decks" had to be known or expected prior to analysis. ${ }^{97}$ Because peaks of interest must be known in order to be transferred to ${ }^{2} \mathrm{D}$ in LC-LC, it is extremely difficult to automate a heart-cutting 2D system. Another potential issue is that 
if the incorrect fraction is collected, peaks of interest could be lost between dimensions. $^{86,98}$

Pandohee et al. used a heart-cutting method with two RP columns in order to separate the constituents of cannabis/hemp plants. This matrix is extremely complex, which complicates work in pharmacological settings where isolation of the pure compounds is important. Fractions of $200 \mu \mathrm{L}$ were collected after they eluted from ${ }^{1} \mathrm{D}$ before injection into ${ }^{2} \mathrm{D}$. Once the sample had gone through separation in both dimensions, another sample could be injected. However, this process resulted in a total analysis time of $12 \mathrm{~h}$, which is not conducive to routine screening, nor does it lend itself to automation. ${ }^{99}$

The separation of samples containing illicit drugs has been improved through the use of LC-LC. Andrighetto et al. used an in silico technique to optimize a 2D separation system with $\mathrm{C}_{18}$ columns in both dimensions in order to differentiate ephedrine and pseudoephedrine-based methamphetamine in seized samples. Following optimization, authentic samples were analyzed and co-eluting peaks were transferred to the second dimension. ${ }^{82}$ However, selection of the peaks to be transferred to the second dimension used simulation data, which presents the risk of missing potential peaks of interest should the simulation be incorrect.

Heart-cutting 2D-LC can be a very valuable tool for improving separation of compounds from complex matrices and mixtures. ${ }^{78}$ The potential peak capacities that can be achieved through heart-cutting methods are impressive, however, there are some clear drawbacks which do not make it an ideal choice for all multi-dimensional separation applications. In particular, LC-LC is not the best choice for the separation of 
mixtures in which there may be unknown targets or in situations where automation is desired. Long analysis times are also common with heart-cutting methods which can slow down productivity. ${ }^{78,86,100-102}$

Alternatively, comprehensive 2D-LC, referred to as LC x LC, offers greatly improved peak capacities as compared to 1D-LC while also allowing for full automation and analysis of the entire sample. Comprehensive 2D-LC methods involve the complete transfer of the ${ }^{1} \mathrm{D}$ effluent to ${ }^{2} \mathrm{D}$ for separation, which is ideal for non-targeted analyses and samples with low concentrations of analytes, since the entire effluent from the first dimension undergoes separation in the second dimension, thus preventing the loss of any sample that might occur when sampling only certain fractions and/or peaks as in LCLC..$^{85,98,103,104}$ Another key benefit to transferring the entire effluent from ${ }^{1} \mathrm{D}$ to ${ }^{2} \mathrm{D}$ is observed when separating extremely complex mixtures such as plant material associated with pharmaceutical or therapeutic samples. In these instances, constituents that are considered to be the target fractions could be even more difficult to isolate because of interferences from endogenous compounds or other interfering analytes in the sample. ${ }^{105}$ The potential for contamination is also minimized in a comprehensive system since samples do not leave the system once injected; typically going from the injection, through the first dimension then into sample loops before elution on to the second dimension. ${ }^{78,85}$

The ability to easily combine LC x LC systems with conventional LC detectors such as MS and UV is also an important advantage over LC-LC methods. Many LC x LC systems include a reversed-phase (RP) column in the second dimension which uses mobile phases conducive to detection with MS. ${ }^{106}$ Using RP columns in each dimension is a popular choice for several 2D-LC applications as result of easier method optimization 
because of mobile phase compatibilities and the ease with which MS detectors can be coupled, resulting in robust methods, particularly for use with pharmaceuticals and derivatives. ${ }^{81,85,97,104,107-109}$

Earlier applications of comprehensive 2D-LC included the use of the first dimension column as an on-line extraction step before achieving chromatographic separation in the second dimension column. Rao and Shinde used a restricted access material (RAM) column followed by a RP $\mathrm{C}_{18}$ column for the $\mathrm{LC} \mathrm{x}$ LC determination of antiretroviral drugs in rat serum and urine. Liquid-liquid extraction (LLE) and solidphase extractions (SPE) were first tried with the samples in an off-line technique, however, acceptable recoveries were not achieved for low concentrations of drugs. These extraction procedures were also tedious to conduct. Comprehensive 2D-LC was then successfully investigated for rat serum and urine samples by employing a RAM column in ${ }^{1} \mathrm{D}$ as an on-line extraction step to remove proteins and large endogenous molecules, followed by chromatographic separation in ${ }^{2} \mathrm{D}$. The method enabled fast extraction and separation of the samples within 20 min with only a filtering step required prior to direct injection into the 2D-LC system. The method was also sensitive enough to detect low $\mathrm{ng} / \mathrm{mL}$ concentrations of the antiretroviral drugs in rat plasma and serum. ${ }^{89}$

Mallet et al. used a comprehensive 2D-LC method, called sequential 2D extraction, in which the first dimension included a sample extraction technique with the purpose of providing decreased sample preparation time and increased separation within a single analysis. A mixture of common illicit drugs and drugs of abuse, including amphetamine, MDMA, mescaline, lidocaine, cocaine, THC, and heroin, were spiked into urine and subjected to the 2D extraction and analysis. The method was successful in 
extracting and detecting the illicit drugs and drugs of abuse at concentrations as low as 10 $\mathrm{pg} / \mathrm{mL}$. Limits of quantitation were set to $1 \mathrm{ng} / \mathrm{mL}$ in a $1 \mathrm{~mL}$ urine sample and the average recovery was $88 \%$ achieved within a 15 min time frame. ${ }^{92}$

The LC x LC methods can also be implemented with analytical columns in each of the dimensions. One such example was presented by Holčapek et al. for the analysis of lipidomic samples. A RP column was placed in the first dimension followed by a hydrophilic interaction chromatography (HILIC) column in the second dimension. The RP column enabled the separation of lipid species using the hydrophobic part of the molecule first, then any co-eluting species were separated in the second dimension on the basis of their differing polarities. Although Holčapek's work was a proof-of-concept study, it did demonstrate the potential of a comprehensive HILIC x RP method for the identification and lipids from human plasma and porcine brain samples. In total, 143 lipid species were identified in a run of $<2.5$ h. ${ }^{110}$

Additional column combinations that have been reported in LC x LC methods, including size exclusion chromatography (SEC) x RP, ion-exchange chromatography (IEX) x SEC, IEX x RP, normal-phase (NP) or HILIC x RP, and HILIC x SEC. However, the most common combination is that of two reversed-phase columns (RP x RP). ${ }^{108}$ Combinations of two RP columns have received a lot of attention because of the potential for high peak capacities and suitability for use with pharmaceuticals and other small molecules. Although combinations using RP columns in each dimension may not be highly orthogonal, variation of mobile phases and gradients can result in desirable peak capacities and resolving power for analytes of interest. ${ }^{111}$ 
Methods using RP x RP have been used for the determination of antioxidants, separation of biological compounds, food analysis, and analysis of natural

products. ${ }^{108,112,113}$ Natural products typically originate from plant materials which present a very complex sample matrix and contain biologically active substances, thus making them of interest for toxicological analyses. Natural products present in Chinese herbal medicine (CHM) are of particular importance. ${ }^{105,114,115}$ The effectiveness of CHMs is believed to come from the combined properties of multiple biologically active components. Therefore CHMs often present a complex mixture of compounds for analysis that requires adequate separation before each can be identified. ${ }^{116}$ For example, Hu et al. developed a LC x LC system for the separation of Rhizoma chuanxiong and Angelica sinensis which represent two of the most commonly used drugs in the prescription of CHM. The method used a cyano $(\mathrm{CN})$ column in the first dimension to separate polar compounds and a silica monolithic ODS column in the second dimension for the separation of the less-polar compounds. About 120 compounds from $R$. chuanxiong and 100 in A. sinensis were successfully separated. ${ }^{115}$

Krieger used an RP x RP separation method in the analysis of Si-Wu-Tang; a CHM made from four different herbs, each with its own set of characteristic compounds that are used for identification purposes. The high complexity and variability within SiWu-Tang make it an ideal choice for separation using 2D-LC. Detection was conducted with QTOF-MS and peaks were matched with library templates for each of the individual compounds. Over $75 \%$ of the template peaks were matched to those detected in $\mathrm{Si}-\mathrm{Wu}-$ Tang, demonstrating the utility of this RP x RP method in the analysis of CHMs. ${ }^{105}$ 
There have been many successful applications of LC x LC, however, the development and optimization of such methods present many challenges. The two dimensions used must be compatible while also being orthogonal. In order to achieve this goal, there are a multitude of parameters in each dimension, including column type and particle size, mobile phase selection, analysis time, gradient, and flow rate, that must first be optimized individually before they are optimized as part of the whole system. $^{73,85,98,108}$

The parameter that can be most influential and the most challenging to optimize is the solvent selection for the mobile phases in each dimension. Solvent selection is particularly difficult when two different types of columns are used in the two dimensions (e.g., normal-phase and reversed-phased) that require different solvent types that may be incompatible..$^{85,98,111}$ If solvents are not compatible, columns could be damaged by the use of improper solvents. A major risk is that compounds separated in the first dimension can remix during transfer to the second dimension, or might not be eluted onto the second dimension at all, and thus 2D separation will not occur. ${ }^{85,106}$

Another potential cause of effluent remixing can occur when the sampling time in the second dimension is longer than the peak width of the effluent transferring from the first dimension, thus causing separable peaks to elute in the same fraction. The discrepancy between sampling time and peak width is referred to as "undersampling" and can lead to decreased peak capacity of the method. Undersampling can be minimized through the appropriate selection of column dimensions for use in the second dimension, as well as optimization of the amount of time set for collection of the effluent from the 
first dimension before transfer to the second, also referred to as the sampling time. $^{78,85,98,117}$

Dilution of an analyte or analytes is an inherent issue of chromatographic methods as the sample disperses along the column. Negative effects of dilution are further compounded when two LC systems are combined, as in 2D-LC. Solvent gradients can be used in each dimension as a way to minimize the effects of sample dilution. ${ }^{74,78}$ In RP $\mathrm{x}$ RP systems, gradients can also be used to improve separation when the columns chosen are not completely orthogonal. ${ }^{111}$ The length of time over which a gradient runs, referred to as the "gradient stop time," is the maximum duration of the gradient in the second dimension. The gradient stop time must always be less than the modulation or sampling time which is equal to the gradient stop time plus the time needed for the second dimension column to equilibrate. ${ }^{86}$

Second dimension gradients in LC x LC are used to generate higher peak capacities, eliminate possible carryover effects, and to improve bandwidth suppression. The samples being analyzed by the 2D-LC system should be taken into consideration whenever a gradient type is chose and optimized. Overall, gradients should be quick and have a steadily increasing slope to allow for better separations. The use of gradients also ensures effective separation of complex mixtures, as the components are likely to have a variety of retention factors that must be accounted for. ${ }^{70,73}$ In $2 \mathrm{D}-\mathrm{LC}$, there are four common gradient types employed in the second dimension (Figure 1); full, segment, shift, and parallel. ${ }^{108}$

Full gradients cover a very steep and wide gradient over the span of a very short amount of time. These gradients provide high bandwidth suppression, which leads to 
greater peak capacities. However, there is a greater chance of carry-over, or wraparound, from compounds that are more strongly retained. Another drawback of using a full gradient, particularly with RP x RP methods, is that compounds with lower (or higher) retention in the first dimension also have lower (or higher) retention in the second dimension. These retention behaviors result in a diagonal appearance of eluted compounds in the final 2D contour plot. ${ }^{108}$

Segment gradients are less steep than full gradients but still have significant bandwidth suppression effects, leading to increased peak capacity. Instead of using a wide, continuous gradient over the entire separation period, a lower gradient coverage is used in the early section of the separation and a higher gradient coverage in the later section. The alteration of gradients throughout the separation, though minimal, results in lower probabilities of wrap-around effects since the gradient range is not continuous as in a full gradient. ${ }^{108}$

A "shifted gradient" is implemented when the second dimension uses a narrow gradient with a changing range or concentration throughout the analysis time. Shifted gradients facilitate compression of peak bandwidths and increased peak capacity in the second dimension. The continuous changing of the gradient minimizes wrap-around effects and demonstrates higher peak capacities than the other gradient types. Through the use of a shifted gradient, more of the 2D separation space can be used, making it a valuable technique for improving separation and spreading peaks further apart. ${ }^{104}$ When a shifted gradient is used, though, there is the possibility that a peak from a single compound might elute in two adjacent separations, thus appearing to have two different 
retention times. Care should be taken to ensure that such peaks are correctly assigned as to one compound. ${ }^{86}$

Parallel gradients are quasi-isocratic gradients which utilize a longer second dimension elution time and eliminate the need for post-gradient equilibration time, but result in larger bandwidth and lower peak capacities. Parallel gradients are best used in accordance with the retention characteristics of the first dimension separation which requires more time and effort to optimize before the gradient can be used effectively. ${ }^{108}$

Second dimension gradients in LC x LC are used to increase peak capacities, eliminate possible carryover effects, and to improve bandwidth suppression. The samples being analyzed by the 2D-LC system should be taken into consideration whenever a gradient type is chosen and optimized. The number of parameters that must be optimized during development of a 2D-LC method is several times greater than the number of parameters in a traditional 1D separation. However, once a comprehensive LC x LC method has been optimized it can be easily automated for use in high throughput applications.

The improved separation and resolving powers offered by 2D-LC can make such methods extremely attractive for the analysis of complex samples and mixtures. Benefits of 2D-LC systems include increased peak capacities, ${ }^{70,103,118}$ separation of isomers, ${ }^{89}$ and increased separation of compounds and metabolites. ${ }^{72,88}$ Other important attributes of 2D-LC include decreased sample preparation time and decreased potential for loss of analytes during preparation as the first dimension can be used to separate proteins and other unwanted substances from analytes, which is especially important in removing endogenous compounds from the biological matrices common in toxicological 
analyses. ${ }^{18,78,88-90,94}$ There are currently no reports in the literature on the separation of coeluting or isomeric NPS using 2D-LC. Examples of existing heart-cutting and comprehensive methods are given in Table 1 (see below). Further investigations into the use of 2D-LC to resolve complex mixtures of NPS in biological specimens is therefore a major goal of the present project.

$=1 \mathrm{D}=2 \mathrm{D}$

A
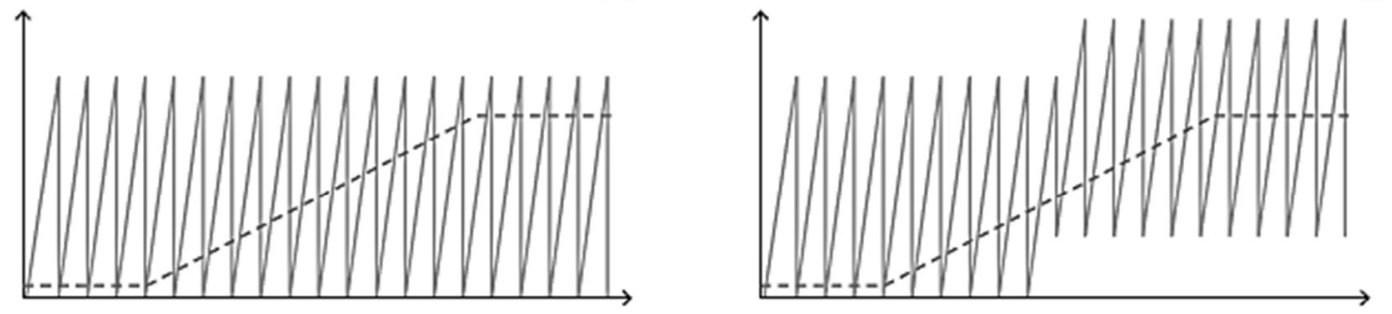

C

D
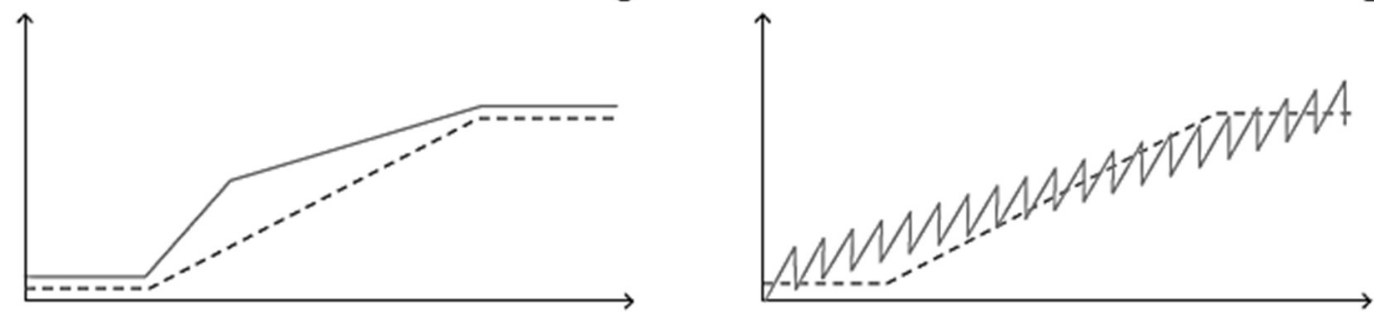

Figure 1. Second-dimension gradient types over time for LC x LC separations: A) full gradient, B) segment gradient, C) parallel gradient, and D) shift gradient. 
Table 1. Examples of 2D-LC method applications found throughout the literature.

\begin{tabular}{|c|c|c|c|c|}
\hline Matrix & 2D-LC Mode & Columns & Detector(s) & Reference \\
\hline $\mathrm{ADCs}$ & $\mathrm{LC} \times \mathrm{LC}$ & $\mathrm{HIC} \times \mathrm{RP}$ & UV-MS & {$[119,120]$} \\
\hline $\mathrm{ADCs}$ & $\mathrm{LC}-\mathrm{LC}$ & $\mathrm{SEC}-\mathrm{RP}$ & DAD; MS & {$[94]$} \\
\hline antibody digest products & $\mathrm{LC} \times \mathrm{LC}$ & $\begin{array}{l}\text { SCX x RP; } \\
\text { RP x RP; } \\
\text { HILIC x RP }\end{array}$ & UV; MS & {$[121]$} \\
\hline antiretroviral drugs & $\mathrm{LC} \times \mathrm{LC}$ & RAM x RP & ion-trap MS & [89] \\
\hline benzenes & LC x LC & $\mathrm{RP} \times \mathrm{RP}$ & UV & {$[122]$} \\
\hline cannabinoids & $\mathrm{LC}-\mathrm{LC}$ & $\mathrm{RP}-\mathrm{RP}$ & ESI-TOF-MS & [99] \\
\hline CHMs & $\mathrm{LC} \times \mathrm{LC}$ & HSA x RP & Ion-trap MS & {$[123]$} \\
\hline drug impurities & $\mathrm{LC} \times \mathrm{LC}$ & $\mathrm{RP} \times \mathrm{RP}$ & $\begin{array}{l}\text { DAD; TOF- } \\
\text { MS }\end{array}$ & {$[124]$} \\
\hline drug metabolites & $\mathrm{LC} \times \mathrm{LC}$ & $\mathrm{RP} \times \mathrm{RP}$ & ESI-MS/MS & {$[72]$} \\
\hline $\begin{array}{l}\text { E. coli and } S \text {. cerevisae metabolomic } \\
\text { products }\end{array}$ & $\mathrm{LC} \times \mathrm{LC}$ & $\begin{array}{l}\text { SCX x } \\
\text { HILIC }\end{array}$ & ESI-MS/MS & {$[88]$} \\
\hline E. coli proteins & $\mathrm{LC} \times \mathrm{LC}$ & $\mathrm{SEC} \times \mathrm{RP}$ & ESI-MS & {$[84]$} \\
\hline EO-PO (co)oligomers & $\mathrm{LC} \times \mathrm{LC}$ & NP x RP & ELSD & {$[125]$} \\
\hline isomeric oligostyrenes & LC x LC & RP x RP & UV & {$[126]$} \\
\hline isomeric oligostyrenes & $\mathrm{LC} \times \mathrm{LC}$ & $\mathrm{RP} \times \mathrm{RP}$ & UV & {$[127]$} \\
\hline lipids & $\mathrm{LC} \times \mathrm{LC}$ & RP x HILIC & ESI-MS/MS & {$[110]$} \\
\hline low MW components of maize & $\mathrm{LC} \times \mathrm{LC}$ & $\mathrm{RP} \times \mathrm{RP}$ & UV & {$[128]$} \\
\hline oligostyrenes & $\mathrm{LC} \times \mathrm{LC}$ & RP x RP & UV & {$[129]$} \\
\hline paracetamol and ketorolac enantiomers & $\mathrm{LC}-\mathrm{LC}$ & RP $x$ chiral & Ion-trap MS & {$[93]$} \\
\hline peptides & $\mathrm{LC}-\mathrm{LC}$ & $\mathrm{RP}-\mathrm{RP}$ & $\begin{array}{l}\text { MALDI-TOF- } \\
\text { MS }\end{array}$ & {$[130]$} \\
\hline peptides & $\mathrm{LC}-\mathrm{LC}$ & $\begin{array}{l}\text { SCX - RP; } \\
\text { RP - RP }\end{array}$ & UV-MS & {$\left[{ }^{131}\right]$} \\
\hline peptides & $\mathrm{LC} \times \mathrm{LC}$ & SEC x RP & ESI-MS & [132] \\
\hline
\end{tabular}




\begin{tabular}{|c|c|c|c|c|}
\hline peptides & LC x LC & RP x HILIC & UV & {$[133]$} \\
\hline pharmaceuticals & mLC - LC & RP - RP & UV & {$[97]$} \\
\hline pharmaceuticals and citrus oils & LC x LC & NP x RP & DAD & {$[134]$} \\
\hline phenolic antioxidants & LC x LC & RP x RP & DAD & {$[135]$} \\
\hline phenolics & LC x LC & HILIC x RP & PDA; ESI-MS & {$[136]$} \\
\hline proteins & LC x LC & IEX x RP & UV; ESI-MS & {$[83]$} \\
\hline sertraline enantiomers & LC x LC & $\begin{array}{c}\text { RAM x } \\
\text { chiral }\end{array}$ & ESI-MS/MS & {$[76]$} \\
\hline steroids, lemon oils & LC x LC/LC & $\begin{array}{c}\text { NP x } \\
\text { RP/RP }\end{array}$ & DAD & {$[137]$} \\
\hline TCMs & LC x LC & RP x RP & $\begin{array}{c}\text { DAD; APCI- } \\
\text { MS }\end{array}$ & {$[107]$} \\
\hline TCMs & LC x LC & RP x RP & DAD & {$[115]$} \\
\hline triacylglycerols in lipids & LC x LC & NP x RP & APCI-MS & {$[138]$} \\
\hline warfarin stereoisomers & LLC - LC & RP x chiral & QTOF-MS & {$[96]$} \\
\hline warfarins/hydroxywarfarins & LC x LC & RP x chiral & ESI-MS/MS & {$[139]$} \\
\hline
\end{tabular}

*mLC - LC $=$ multiple heart-cutting 


\section{DEVELOPMENT OF DATABASE AND SPECTRAL LIBRARY}

\subsection{Introduction}

The presence of novel psychoactive substances (NPS) on the illicit drug market and therefore present in toxicological specimens is not a new occurrence. However, as NPS continue to be developed, analytical methods for detection and identification of such

compounds must adapt to keep pace. ${ }^{140,141}$ Novel psychoactive substances are altered by illicit manufacturers to circumvent federal, state, and local legislation intended to control their usage in public. Preventing a substance from falling under legal control can be achieved through an action as simple as altering a functional group through its removal, addition, or movement along the chemical structure. As these changes are made, the structures of the resultant compounds no longer match those of substances that are illicit, thus placing them outside the purview of the controlled substance laws. ${ }^{2,5,18}$

The constant emergence of new NPS also presents analytical challenges, since existing methods of detection are typically designed for specific functional groups or structures. ${ }^{6}$ Identification of analytes of interest in samples generally begins with the use of a screening technique to tentatively identify possible compounds or classes of compounds present in the sample. ${ }^{45,63,142}$ Methods using GC-MS and LC-MS have been developed for screening purposes but both typically require the use of libraries, which contain characteristic mass spectra generated using electron ionization (EI) or electrospray ionization (ESI) techniques, respectively, to make identifications.

Using characteristic mass spectra to identify analytes in a sample is not a new concept. Libraries exist containing spectra for as few as a couple dozen compounds to as many as several hundreds of thousands. . $^{78,49,53,143}$ The most common libraries contain 
spectra generated through EI, because of their high degree of reproducibility and because EI-based libraries have been established for longer than those that are ESI-based. Libraries containing spectra from ESI sources have been increasing in popularity among forensic toxicology laboratories. ${ }^{47,53,56,144,145}$ There are several benefits to using ESI over EI, including the ability to maintain an intact molecular ion, which leads to increased confidence in identification because of the ionization in ESI being a "softer" or less intense technique than EI. Techniques using ESI are also not limited to use with only volatile, thermally stable molecules as with EI sources in GC. ${ }^{42,52}$ Currently, there exist a number of GC libraries, but large LC libraries are lacking, and those that do exist do not contain many NPS or use theoretical accurate masses rather than masses measured from reference standards..$^{7-9}$

Development of ESI-based libraries for LC methods has historically involved the use of triple quadrupole mass spectrometry (QqQ-MS) but these instruments are considered to have low resolution, which makes differentiation of some NPS difficult, particularly those with extremely similar accurate masses. ${ }^{28,40}$ In contrast, highresolution mass spectrometry (HRMS) techniques, such as those using hybrid quadrupole time-of-flight mass spectrometers (QTOF-MS), are generally sensitive enough to distinguish between compounds with minute differences in accurate mass. ${ }^{40,66}$ Another benefit to using HRMS techniques is the ability to collect information for all ions while still maintaining high resolution and mass accuracy, which is ideal for comprehensive screening. Collection of all ion data also allows for retrospective screening without the need for reanalysis of the sample; allowing for newly reported compounds to be searched for in previously analyzed samples..$^{40,46,146}$ 
The present study reports the development of a comprehensive compound database for 499 unique chemical entities considered to be possible NPS, metabolites, and related compounds. A full HRMS spectral library was created for 410 of these compounds, with partial spectral information for another 25 compounds also included. A comprehensive compound database was also created for 76 deuterated internal standard compounds.

\section{Materials and Methods}

\subsubsection{Chemicals}

Optima LCMS grade methanol (MeOH), acetonitrile (ACN), HPLC water, and formic acid were purchased from Fisher Scientific (Fair Lawn, NJ, USA). Ammonium formate (99\%) was also purchased from Fisher Scientific.

The ESI-L tuning mix (p/n: G1969-85000) and $0.1 \mathrm{mM} \mathrm{HP-0321} \mathrm{(I8720263)}$ were obtained from Agilent Technologies (Santa Clara, CA, USA) and used to prepare the tuning solution for the LC-QTOF-MS.

\subsubsection{Standards}

Reference standards for the NPS and internal standards were obtained from Cayman Chemical (Ann Arbor, MI, USA). Standards that were received as neat solid material were put into solution with methanol $(\mathrm{MeOH})$ for storage at $-20^{\circ} \mathrm{C}$. Compounds that were not readily soluble in $\mathrm{MeOH}$ were put into solution with dimethyl sulfoxide (DMSO). Each standard was assigned a unique identifying number for in-house usage (i.e., FIU-nnnn) in order to track usage of the compounds throughout sample preparation and analysis. 
An arginine reference standard was also obtained from Cayman Chemical for use as a quality control standard.

\subsubsection{Sample Preparation}

Reference solutions were prepared from the reference standards of 499 NPS and 76 internal standards at concentrations of $10 \mu \mathrm{g} / \mathrm{mL}$ in $\mathrm{MeOH}$. Working solutions were then prepared from the reference solutions at concentrations of $1 \mu \mathrm{g} / \mathrm{mL}$ in $\mathrm{MeOH}$. A 1.6 $\mathrm{ng} / \mu \mathrm{L}$ working solution of arginine was prepared in HPLC water for use as a quality control to ensure proper tuning and calibration of the instrument prior to FIA.

\subsubsection{Instrumentation and Software}

Instrumentation used for analysis included an Agilent 1290 Infinity UHPLC system coupled to an Agilent 6530 Accurate-Mass QTOF-MS (Agilent Technologies, Santa Clara, CA, USA). A Zorbax Eclipse Plus $\mathrm{C}_{18}$ Rapid Resolution HD column (2.1 x 50 mm, $1.8 \mu \mathrm{m}$; Agilent Technologies, Santa Clara, CA, USA) was used for separation during the database and library applicability test. The QTOF-MS was operated in positive-ion electrospray mode with Jet Stream ESI technology.

Agilent MassHunter LC/MS Acquisition software for the 6200 series TOF/6500 series QTOF (Version B.06.00) was used to acquire spectral data. MassHunter Qualitative Analysis software (Version B.06.00) was used to process the data. MassHunter Personal Compound Database Library (PCDL) Manager software (Version B.07.00, Build 7024.0) was used to create the compound database and high-resolution MS/MS spectral library. ChemBioDraw Ultra (Version 14.0.0.117; PerkinElmer, 
Waltham, MA, USA) was used to create the 2D chemical structure of each NPS for use in the PCDL.

\subsubsection{Methods}

Collection of spectral data for the MS/MS spectral library was done via flow injection analysis (FIA). Diluted standards were individually injected directly into an Agilent 6530 series Accurate-Mass Quadrupole Time-of-Flight (QTOF) mass spectrometer with Jet Stream ESI ion source coupled to an Agilent 1290 Infinity Series Binary Pump system. Most injection volumes were $1 \mu \mathrm{L}$, however, some compounds had to be injected at volumes up to $10 \mu \mathrm{L}$ to produce spectra with base peak intensities

over the 1000 count threshold. A small percentage of compounds also had to be injected at concentrations of $2 \mu \mathrm{g} / \mathrm{mL}$.

A 50:50 isocratic mobile phase system was used at a flow rate of $0.2 \mathrm{~mL} / \mathrm{min}$ for 2 min with aqueous (A) $5 \mathrm{mM}$ ammonium formate in HPLC water with $0.1 \%$ formic acid, and organic (B) acetonitrile with $0.1 \%$ formic acid. A positive mode electrospray ionization (ESI) targeted MS/MS method was used to collect the data. The quadrupole used a narrow isolation mass window of $1.3 \mathrm{amu}$. The ESI source parameters were: drying gas temperature $325^{\circ} \mathrm{C}$; drying gas flow rate $5 \mathrm{~L} / \mathrm{min}$; nebulizer 30 psi; sheath gas temperature $375^{\circ} \mathrm{C}$; sheath gas flow $12 \mathrm{~L} / \mathrm{min}$. Scanning source parameters were: VCap voltage $4000 \mathrm{~V}$; nozzle voltage $0 \mathrm{~V}$; fragmentor voltage $140 \mathrm{~V}$; skimmer voltage $65 \mathrm{~V}$.

Compounds were fragmented at three standard collision energies $(10,20$, and 40 eV) to produce characteristic fragmentation patterns. The MS range was set to 50-1700 $m / z$ with an MS acquisition rate of 10 spectra per second. The MS/MS range was set to 
25-1700 m/z with an MS/MS acquisition rate of 3 spectra per second. Spectral data were added to the MS/MS spectral library when the compound fragmentation produced a base peak of at least 1000 counts and had a mass accuracy within $5 \mathrm{ppm}$. If these criteria were met, the compound information and fragment ion spectrum from each collision energy was imported into the PCDL using PCDL Manager software. An arginine standard was run with each batch of standards to ensure that the instrument was properly tuned and calibrated.

The "Find by Formula" (FBF) function of the Qualitative Analysis software was used to isolate a targeted compound from the FIA chromatogram. The MS/MS spectra were then extracted and exported into the PCDL using the "Send Spectra to PCDL" function.

\subsection{Results and Discussion}

Development of libraries for use with ESI-based methods are typically generated using QqQ-MS because of the greater availability of such instrumentation in laboratories. In these instruments, the first quadrupole (Q1) is typically used to scan for a specific precursor ion or range of ions of interest. The selected ions then move to a collision cell (Q2) where they are fragmented using a neutral collision gas. These ion fragments then pass to the third quadrupole $(\mathrm{Q} 3)$ where selected fragment ions, or product ions, are allowed to pass to the detector while all other ions are filtered out. ${ }^{79}$ The fragmentation of ions, or transitions, from the precursor to the product ions is recorded using multiple reaction monitoring (MRM). These MRM transitions are included in QqQ-MS libraries and serve as the characteristic fragmentation data for identification of compounds. However, the collision energy required to generate these characteristic MRM transitions 
must be optimized for each compound prior to collection. The need for optimization introduces another step and requires more time to add new compounds to the library. Libraries of MRM transitions also have low degrees of resolution as compared to HRMS techniques, which may result in significant challenges when trying to differentiate between compounds with similar accurate masses. ${ }^{66,146}$

Building a library using an HRMS technique such as QTOF-MS does not require collision energies and fragmentor voltages to be optimized prior to data collection. Instead, multiple collision energies can be employed and collection of all resultant spectra can be conducted simultaneously. The capability to comprehensively collect spectra makes the rapid addition of spectra for new compounds possible and much easier to do than with MRM transition libraries. The ability to collect high resolution full scan MS and MS/MS fragmentation data presents a large advantage for QTOF-MS instrumentation over QqQ-MS, since information about potential unknown compounds can be collected in addition to the high mass accuracy information of known compounds.

In the present research, the major goal was to create an HRMS spectral library for as many NPS standards from the synthetic cannabinoid, stimulant, hallucinogen, and other related classes using a QTOF-MS approach (Table 2 displays the number of compounds represented from each class of NPS; Table 3 displays the types of molecules included in the database). These spectra were later combined with an existing HRMS library containing spectral data for an additional 260 compounds. ${ }^{147}$ The new compound database was first created using the PCDL software. This database contained information for 499 entries including NPS, metabolites, and related compounds. A second database was also created containing entries for 76 deuterated internal standards (see Appendices 1 
and 2 for a complete listing of compounds contained in each respective database).

Compounds selected for inclusion in the database were chosen from a variety of sources, including lists of commercially available standards, government documents and reports, scientific literature, and online drug-user forums. Standard information input into the database included the compound common name, the IUPAC name, the molecular formula, calculated accurate mass, a 2D structure, as well as the CAS registry and ChemSpider numbers when available. Reference standards for each entry were obtained from Cayman Chemical and the manufacturer's product number was also included in the entry for traceability. Figure 2 presents an example of the compound database generated using the MassHunter PCDL software with information for the compound acetyl fentanyl displayed. Under the column labeled "Spectra", the number of successfully acquired HRMS spectra for that compound is presented.

Table 2. Distribution of compounds in database by class.

\begin{tabular}{|c|c|}
\hline Drug Class & Number in Database \\
\hline Synthetic Cannabinoid & 295 \\
\hline Other* & 89 \\
\hline Cathinone & 67 \\
\hline Phenethylamine & 29 \\
\hline Tryptamine & 14 \\
\hline Piperazine & 5 \\
\hline
\end{tabular}

*includes opioids, amphetamines, benzofurans, and other compounds. 
Table 3. Distribution of compounds in database by molecule type.

\begin{tabular}{|c|c|}
\hline Molecule Type & Number in Database \\
\hline Precursor Compound & 293 \\
\hline Metabolite & 109 \\
\hline Isomers & 71 \\
\hline Analogs* & 21 \\
\hline Glucuronides & 5 \\
\hline
\end{tabular}

*derivatives of NPS that are not considered metabolites or isomers.

High resolution mass spectra were generated by direct injection of $1 \mu \mathrm{L}$ volumes of the 435 individual compound solutions into the ESI source of the QTOF-MS at concentrations of $1 \mu \mathrm{g} / \mathrm{mL}$ in $\mathrm{MeOH}$. After preparation in $\mathrm{MeOH}$, the compounds were ionized in positive mode and targeted ions were subjected to three standard collision energies $(10,20$, and $40 \mathrm{eV})$. All resultant MS/MS spectral data were collected then processed to determine suitability for inclusion into the database. Once MS/MS spectral data were included in the database, it was more properly referred to as the HRMS spectral library.

In order to be accepted into the library, there were several criteria that the collected data had to meet. For each compound data file, the "Find by Formula" (FBF) function in MassHunter Qualitative Analysis was used to isolate the individual compound. The FBF function was linked to the compound database which assigned identification using the accurate mass and generated chemical formula. A secondary function within FBF was the "Extract MS/MS spectrum" function. Using this, the MS/MS spectrum of the compound at each collision energy was extracted. For inclusion in the HRMS spectral library, compounds identified using FBF needed to have a database search "score" $>90$ and a mass accuracy within \pm 5 ppm. The match "score" of a 
compound is generated through the use of both a forward search (when data in the sample are matched against those in the library) and a reverse search (when library data are searched against data in the sample). The MS/MS spectral data at each collision energy were then required to have base peak counts of at least 1000 to avoid inclusion of ion peaks from the background. A small percentage of compound solutions needed to be injected at concentrations of $2 \mu \mathrm{g} / \mathrm{mL}$ or at volumes up to $10 \mu \mathrm{L}$ in order to meet these criteria for inclusion in the HRMS spectral library.

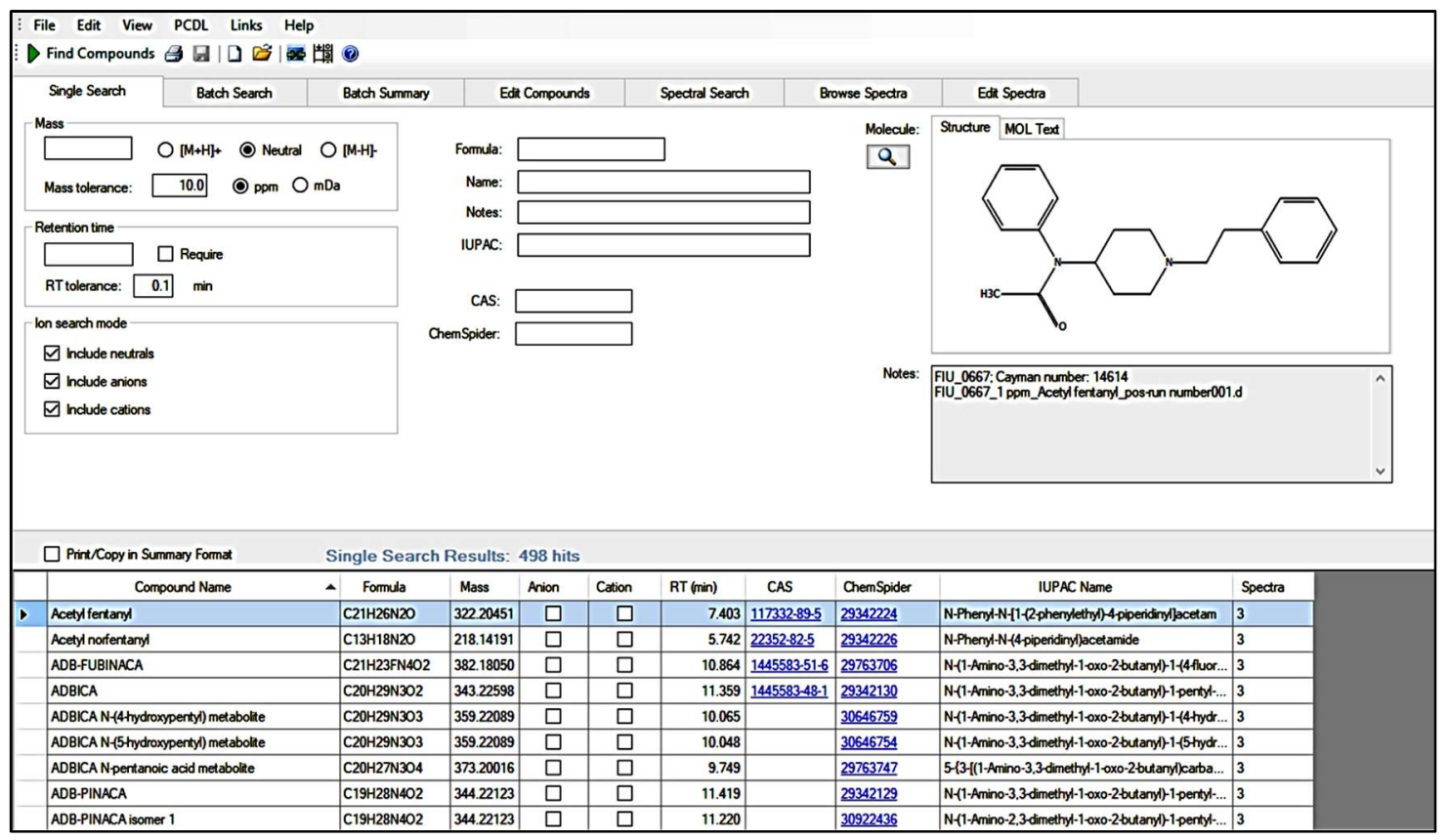

Figure 2. An image of the PCDL software used to create the compound database and MS/MS spectral library.

On the basis of the FBF scores $>90$, mass accuracy within \pm 5 ppm, and the 1000 count MS threshold, full MS/MS spectral information at all three collision energies was included in the library for 410 of the 435 NPS, metabolites, and related compounds. A representation of collected MS/MS spectra for the compound acetyl fentanyl is presented 
in Figure 3. Of the remaining 25 compounds, MS/MS spectra collected at two of the three collision energies were included for 19 compounds with another six compounds having only a single acceptable MS/MS spectrum. The ions represented at relative abundances $>10 \%$ for each MS/MS spectrum accepted into the library are presented in Appendix 3.

\subsection{Conclusion}

The compound database and HRMS spectral library were successfully created containing approximately 550 compounds with MS/MS spectral data for over 470 compounds at three distinct collision energies $(10,20$, and $40 \mathrm{eV})$. Retention time data were also included in the database for in-house use to help differentiate among compounds with similar fragmentation patterns. The database and library were combined with another library that had been created as part of a previous project which included MS/MS spectra for an additional 260 compounds, bringing the number of compounds in the library with MS/MS data to over 700. Entries for which complete MS/MS spectra could not be collected were still included in the database to be used for compound identification using accurate mass data. Ultimately, over 800 compounds were represented in the database.

The high resolution and high mass accuracy of LC-QTOF-MS presents a significant advantage for screening and confirmation of NPS with a high degree of confidence in correct identification. Through the use of a MS/MS spectral library, the confidence in identification is further increased. Further work was conducted to evaluate the practicality of this technique for routine forensic toxicological screening of NPS following standard validation parameters. 

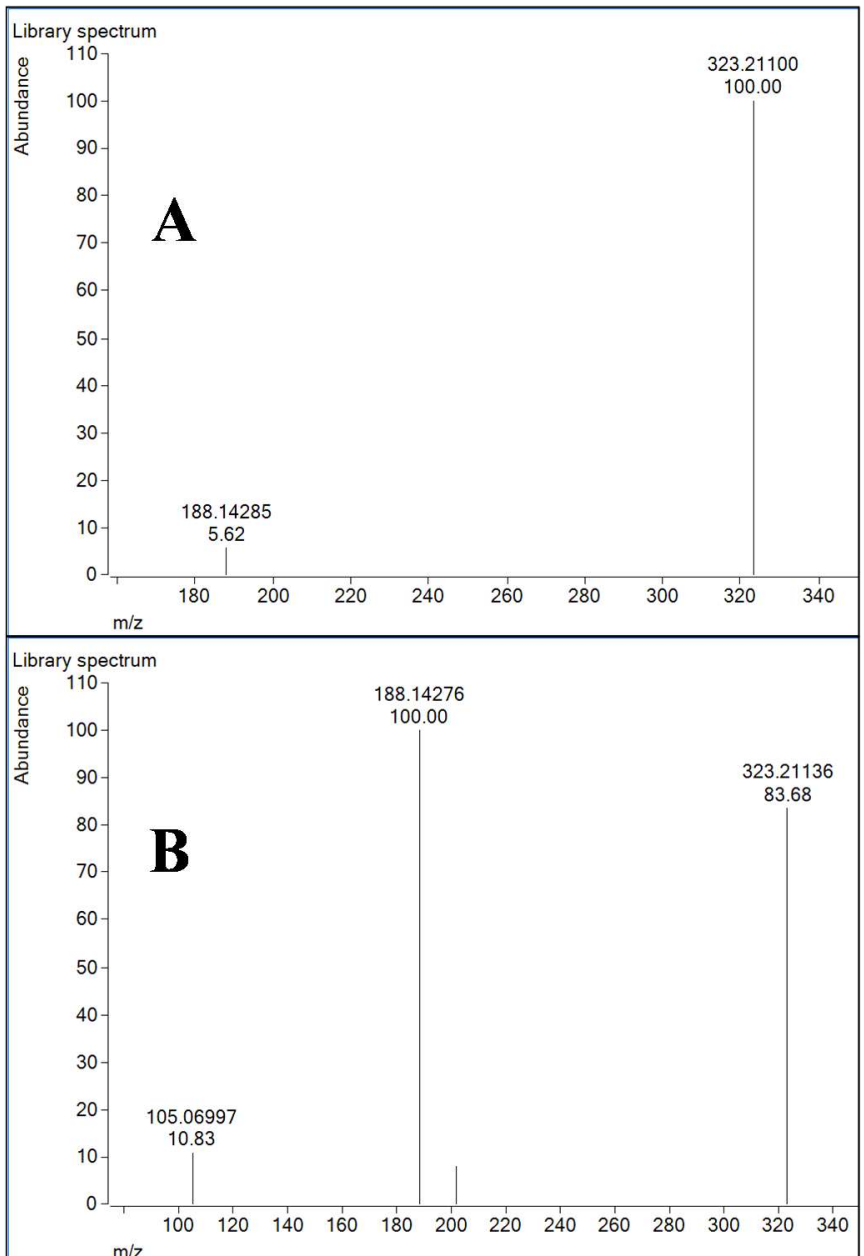

$\mathrm{m} / \mathrm{z}$

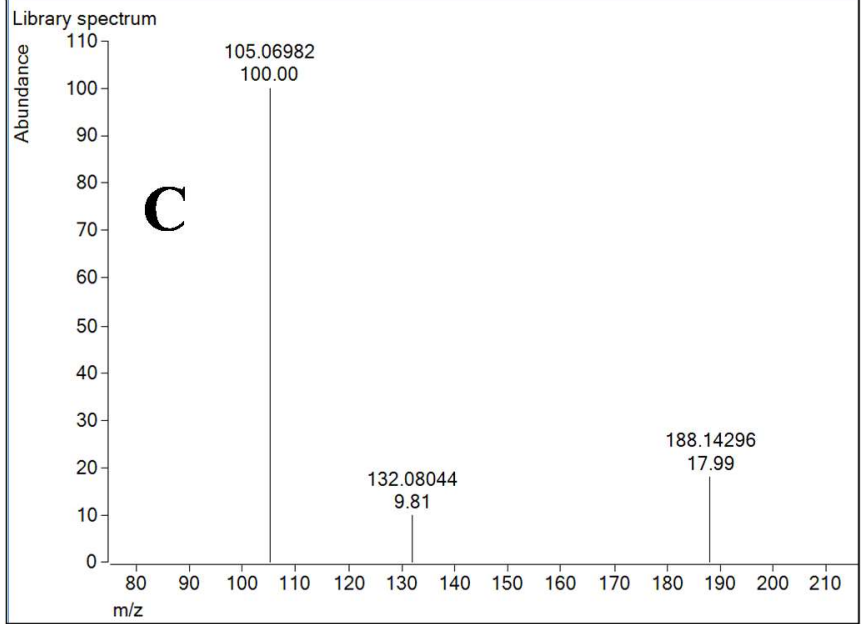

Figure 3. Images of PCDL software showing the MS/MS spectral data of acetyl fentanyl. MS/MS spectral data is shown at collision energies: A) $10 \mathrm{eV}$, B) $20 \mathrm{eV}$, and C) $40 \mathrm{eV}$. 


\section{COMPREHENSIVE LC-QTOF-MS METHOD VALIDATION}

\subsection{Introduction}

Before the developed compound database and HRMS spectral library can be implemented for routine comprehensive screening, the method to be used in conjunction with the library must be fully validated. Validation ensures that analysis using the method produces reliable data that are not false or susceptible to misinterpretation. In clinical and forensic toxicology, unreliable data may be contested in court and could also result in mistreatment of patients or improper consequences of defendants in legal proceedings. ${ }^{148}$

Standard validation practices have been set forth by the Toxicology Subcommittee of the Organization of Scientific Area Committees (OSACs). These standard practices evolved from previously established standard practices from the Scientific Working Group for Forensic Toxicology (SWGTOX) which disbanded in 2014. The SWGTOX guidelines drew heavily from work published by Peters in 2007, who recommended a series of experiments to validate a method including the following criteria; selectivity, linearity, accuracy (bias), precision, and the limit of quantitation (LOQ). Other parameters such as the limit of detection (LOD), recovery, reproducibility, ruggedness, stability, and matrix effects were also suggested by Peters for inclusion in method validation procedures. ${ }^{148,149}$

A goal of the present work was to validate a method for screening and confirmation of over 800 NPS and related compounds. In order to do so, validation experiments were initially designed following the SWGTOX guidelines and later updated following the release of the OSAC guidelines. ${ }^{150}$ Validation experiments for the present 
work were conducted for the following parameters; linearity, accuracy, precision, LOD, LOQ, freeze/thaw stability, matrix effects, and carryover.

Validation of a method for toxicological screening and confirmation is typically conducted for a single class of compounds or for a small set of compounds, as the process can require a significant amount of time and resources. In the present work, method validation was conducted using a mixture approach in which each mixture of standards was validated as if it were a single compound. Three validation mixtures were created, each containing between 27-33 compounds, and validated according to the guidelines set by the Toxicology Subcommittee of OSAC.

The validated method was also used in the qualitative investigation of the applicability of the developed compound database and HRMS library for screening of blind-spiked urine specimens. This investigation used 20 blank urine samples that were spiked with 0-1 NPS represented in the database and library. The results of this qualitative investigation indicated that the database and library were suitable for screening purposes.

\subsection{Materials and Methods}

\subsubsection{Chemicals}

Optima LCMS grade methanol (MeOH), HPLC water, and formic acid were purchased from Fisher Scientific (Fair Lawn, NJ, USA). Ammonium formate (99\%) was also purchased from Fisher Scientific.

The ESI-L tuning mix (p/n: G1969-85000) and 0.1 mM HP-0321 (I8720263) were obtained from Agilent Technologies (Santa Clara, CA, USA) and used to prepare the tuning solution for the ESI-QTOF-MS. 
A reference mass solution to ensure proper instrumental calibration throughout the analyses was created using the ESI TOF Mass Kit (p/n: G1959-85001) obtained from Agilent Technologies. The kit contained $100 \mathrm{mM}$ ammonium trifluoroacetate $\left(\mathrm{TFANH}_{4}\right)$ in $\mathrm{ACN}: \mathrm{H}_{2} \mathrm{O}(90: 10), 5 \mathrm{mM}$ purine in $\mathrm{ACN}: \mathrm{H}_{2} \mathrm{O}(90: 10)$, and $2.5 \mathrm{mM}$ hexakis in $\mathrm{ACN}: \mathrm{H}_{2} \mathrm{O}(90: 10)$.

\subsubsection{Standards}

Reference standards used in the method validation are the same as those used in creation of the compound database and HRMS spectral library for both the compounds and for the deuterated internal standards. All reference standards were obtained from Cayman Chemical (Ann Arbor, MI, USA).

\subsubsection{Preparation of Mixtures}

The same working solutions that were prepared for the creation of the compound database and HRMS library were used for method validation. The working solutions contained individual reference standards at concentrations of $10 \mu \mathrm{g} / \mathrm{mL}$ in $\mathrm{MeOH}$. Three validation mixtures were created containing 29, 28 and 33 individual compounds, respectively, with each compound present at a concentration of $200 \mathrm{ng} / \mathrm{mL}$ in $\mathrm{MeOH}$. The identity of the compounds contained in each mixture are displayed in Table 4, Table 5, and Table 6. The mixtures were designed so that compounds from a variety of NPS classes were represented in each and that no two compounds in a single mixture exhibited co-elution.

An additional mixture was created to serve as the internal standard in each validation mixture for quantitation purposes. The internal standard (IS) mixture 
contained 22 deuterated internal standards representing a variety of NPS classes, each present in the mixture at a concentration of $200 \mathrm{ng} / \mathrm{mL}$ in $\mathrm{MeOH}$. The compounds contained in the IS mixture are presented in Table 7. A compound from the IS mixture was matched with each NPS in the validation mixtures for quantitation purposes. These "matches" were based on similarities between the NPS and IS compound structures since the IS should be chemically similar to the analyte of interest. The IS compounds used in this research were chosen to represent a variety of drugs, with the majority from the SC class of compounds due to the relevance of SC in real-world samples as well as the large representation of SC in the database and library developed prior to the validation studies.

To validate the method in matrix, blank, pooled human urine was obtained from UTAK Laboratories (Valencia, CA, USA). For all analyses in matrix, the urine was diluted with aqueous mobile phase at a 1:5 dilution.

\subsubsection{Instrumentation and Software}

Instrumentation used for analysis included an Agilent 1290 Infinity UHPLC system coupled to an Agilent 6530 Accurate-Mass QTOF-MS (Agilent Technologies, Santa Clara, CA, USA). A Zorbax Eclipse Plus $\mathrm{C}_{18}$ Rapid Resolution HD column (3.0 x $100 \mathrm{~mm}, 1.8 \mu \mathrm{m}$; Agilent Technologies, Santa Clara, CA, USA) was used for separation of compounds in the urine matrix. The QTOF-MS was operated in positive-ion electrospray mode with Jet Stream ESI technology.

Agilent MassHunter LC/MS Acquisition software for the 6200 series TOF/6500 series QTOF (Version B.06.00) was used to acquire the data. MassHunter Qualitative Analysis software (Version B.06.00) was used to process acquired retention time data and 
to process the blind-spiked urine specimens. MassHunter Quantitative Analysis software for QTOF (Version B.07.00, Build 7.0.457.0) was used for quantitation of the data. The compound database and HRMS spectral library created and managed using MassHunter PCDL software (Version B.06.00) was used to identify compounds in the blind-spiked urine specimens. 
Table 4. Compounds contained in validation Mix 1, their accurate masses, and retention times.

\begin{tabular}{|c|c|c|}
\hline Compound Name & Accurate Mass (Da) & $\begin{array}{c}\text { Retention Time } \\
\text { (min) }\end{array}$ \\
\hline 25I-NBMD & 441.0437 & 9.17 \\
\hline 3-Methylbuphedrone & 191.1310 & 7.29 \\
\hline 4-APDB & 177.1154 & 6.43 \\
\hline 4-fluoro- $\alpha$-Pyrrolidinobutiophenone & 235.1372 & 6.83 \\
\hline 4'-fluoro- $\alpha$-Pyrrolidinopropiophenone & 221.1216 & 6.17 \\
\hline 4-hydroxy MET & 218.1419 & 5.21 \\
\hline 4-methoxy PV8 & 289.2042 & 8.97 \\
\hline 4-methoxy- $\alpha$-Pyrrolidinopentiophenone & 261.1729 & 7.70 \\
\hline 4-Methyl- $\alpha$-ethylaminobutiophenone & 205.1467 & 7.49 \\
\hline 5-fluoro SDB-006 & 338.1794 & 10.80 \\
\hline 5-Fluoropentylindole & 205.1267 & 11.21 \\
\hline 5-MAPB & 189.1154 & 6.99 \\
\hline $\mathrm{AB}-005$ & 352.2515 & 9.58 \\
\hline AM-2233 azepane isomer & 458.0855 & 8.66 \\
\hline AM694 N-(5-hydroxypentyl) metabolite & 433.0539 & 10.45 \\
\hline AMT & 130.0565 & 1.63 \\
\hline Benocyclidine & 299.1708 & 9.14 \\
\hline Flubromazepam & 331.9961 & 10.07 \\
\hline JWH 031 2'-isomer & 305.1780 & 13.32 \\
\hline JWH 081 N-(5-hydroxypentyl) metabolite & 387.1834 & 10.94 \\
\hline JWH 200 5-hydroxyindole metabolite & 400.1787 & 8.61 \\
\hline JWH 203 & 339.1390 & 12.22 \\
\hline N-Methyltryptamine & 174.1157 & 5.73 \\
\hline NPB-22 & 359.1634 & 11.53 \\
\hline PB-22 6-hydroxyisoquinoline isomer & 358.1681 & 11.98 \\
\hline PCMPA & 247.1936 & 8.25 \\
\hline THCA-A & 358.2144 & 19.14 \\
\hline UR-144 N-heptyl analog & 339.2562 & 15.45 \\
\hline$\alpha$-Pyrrolidinopentiophenone metabolite 1 & 233.1780 & 7.65 \\
\hline
\end{tabular}


Table 5. Compounds contained in validation Mix 2, their accurate masses, and retention times.

\begin{tabular}{|c|c|c|}
\hline Compound Name & Accurate Mass (Da) & $\begin{array}{c}\text { Retention } \\
\text { Time (min) }\end{array}$ \\
\hline 2,3-methylenedoxy pyrovalerone & 275.1521 & 7.39 \\
\hline $25 \mathrm{I}-\mathrm{NBF}$ & 415.0445 & 9.11 \\
\hline $2 \mathrm{C}-\mathrm{T}-2$ & 241.1136 & 7.90 \\
\hline $2 \mathrm{C}-\mathrm{T}-4$ & 255.1293 & 8.51 \\
\hline 2-fluoromethcathinone & 181.0903 & 5.22 \\
\hline 3,4-DHMA & 181.1103 & 4.21 \\
\hline $\begin{array}{c}\text { 3,4-dimethoxy- } \alpha- \\
\text { pyrrolidinopentiophenone }\end{array}$ & 291.1834 & 7.19 \\
\hline $\begin{array}{c}\text { 3-methyl- } \alpha \text { - } \\
\text { pyrrolidinopropiophenone }\end{array}$ & 217.1467 & 6.93 \\
\hline $\begin{array}{c}\text { 4'-methyl-N- } \\
\text { methylhexanophenone }\end{array}$ & 219.1623 & 8.56 \\
\hline 4-ethyl-N,N-dimethylcathinone & 205.1467 & 7.44 \\
\hline 4-fluoroisocathinone & 167.0746 & 5.66 \\
\hline 4-hydroxy MiPT & 232.1576 & 5.67 \\
\hline 4-MMC & 177.1154 & 6.57 \\
\hline A-796260 & 354.2307 & 10.90 \\
\hline AB-005 azepine isomer & 352.2515 & 9.78 \\
\hline $\begin{array}{l}\text { AB-FUBINACA 3-fluorobenzyl } \\
\text { isomer }\end{array}$ & 368.1649 & 10.49 \\
\hline ADB-PINACA isomer 1 & 344.2212 & 11.20 \\
\hline AKB48 N-(5-fluoropentyl) analog & 383.2373 & 13.22 \\
\hline Clencyclohexerol & 318.0902 & 6.10 \\
\hline EG-018 & 391.1936 & 16.98 \\
\hline $\begin{array}{l}\text { JWH } 018 \text { N-(5-hydroxypentyl) } \\
\text { metabolite }\end{array}$ & 357.1729 & 10.74 \\
\hline $\begin{array}{l}\text { JWH } 018 \text { N-propanoic acid } \\
\text { metabolite }\end{array}$ & 343.1208 & 10.31 \\
\hline KM 233 & 362.2246 & 14.08 \\
\hline Loperamide & 476.2231 & 9.52 \\
\hline $\begin{array}{l}\text { MAM2201 N-pentanoic acid } \\
\text { metabolite }\end{array}$ & 385.1678 & 10.99 \\
\hline N-Ethylbuphedrone & 191.1310 & 6.53 \\
\hline PB-22 & 346.1681 & 11.88 \\
\hline $\mathrm{PCPr}$ & 217.1830 & 8.35 \\
\hline RCS-4 2-methoxy isomer & 321.1729 & 11.60 \\
\hline SER-601 & 434.2933 & 15.46 \\
\hline UR-144 Degradant & 311.2249 & 12.97 \\
\hline
\end{tabular}




\begin{tabular}{|c|c|c|}
\hline XLR11 N-(2-fluoropentyl) isomer & 329.2155 & 12.46 \\
\hline$\Delta 8$-THC & 314.2246 & 14.79 \\
\hline
\end{tabular}


Table 6. Compounds contained in validation Mix 3, their accurate masses, and retention times.

\begin{tabular}{|c|c|c|}
\hline Compound Name & Accurate Mass (Da) & $\begin{array}{c}\text { Retention } \\
\text { Time (min) }\end{array}$ \\
\hline (R)-(-)-MT-45 & 348.2565 & 9.48 \\
\hline 2,3-Dichlorophenylpiperazine & 230.0378 & 8.31 \\
\hline $25 \mathrm{H}-\mathrm{NBOMe}$ & 301.1678 & 8.41 \\
\hline $2 \mathrm{C}-\mathrm{T}$ & 227.0980 & 7.50 \\
\hline 2-Methoxyamphetamine & 165.1154 & 7.95 \\
\hline 3,4-Dimethylethcathinone & 205.1467 & 7.64 \\
\hline $3 \mathrm{C}-\mathrm{P}$ & 253.1678 & 8.10 \\
\hline 4-Methoxyamphetamine & 165.1154 & 7.42 \\
\hline 5-fluoro NNEI & 374.1794 & 11.09 \\
\hline 9-octadecenamide/oleamide & 281.2719 & 14.77 \\
\hline AB-CHMINACA & 356.2212 & 11.50 \\
\hline AKB48 N-pentanoic acid metabolite & 395.2209 & 11.81 \\
\hline BB-22 4-hydroxyquinoline isomer & 384.1838 & 13.46 \\
\hline Cathine & 151.0997 & 5.50 \\
\hline Diclofensine & 321.0687 & 9.87 \\
\hline FUB-PB-22 & 396.1274 & 11.29 \\
\hline HMA & 181.1103 & 5.11 \\
\hline JWH 018 2-hydroxyindole metabolite & 357.1729 & 15.25 \\
\hline JWH 251 3-methylphenyl isomer & 319.1936 & 12.51 \\
\hline MBZP & 190.1470 & 5.47 \\
\hline Mephedrone & 177.1154 & 6.58 \\
\hline Methylenedioxy Pyrovalerone metabolite 2 & 263.1521 & 5.92 \\
\hline N-methyl-2-AI & 147.1048 & 5.83 \\
\hline NRG-3 & 241.1467 & 8.76 \\
\hline PB-22 N-(5-hydroxypentyl) metabolite & 374.1630 & 10.45 \\
\hline RCS-4 N-(4-hydroxypentyl) metabolite & 337.1678 & 10.33 \\
\hline UR-144 N-(2-chloropentyl) analog & 345.1859 & 13.07 \\
\hline$\triangle 9-\mathrm{THC}$ & 314.2246 & 14.44 \\
\hline
\end{tabular}


Table 7. Compounds contained in the internal standard (IS) mixture and their accurate masses.

\begin{tabular}{|c|c|c|}
\hline Compound & $\begin{array}{c}\text { Accurate } \\
\text { Mass (Da) }\end{array}$ & $\begin{array}{c}\text { Retention } \\
\text { Time (min) }\end{array}$ \\
\hline (-)-11-nor-9-carboxy- $\Delta^{9}-\mathrm{THC}-\mathrm{d} 3$ & 347.2176 & 12.57 \\
\hline ( \pm )-CP 47,497-C8-homolog-d7 & 339.3155 & 14.49 \\
\hline 25I-NBOMe-d3 & 430.0833 & 9.39 \\
\hline 3,4-Methylenedioxy pyrovalerone-d8 & 283.2024 & 7.51 \\
\hline AB-FUBINACA-d4 & 372.1900 & 10.62 \\
\hline AB-PINACA-d9 & 339.2621 & 11.18 \\
\hline Acetyl norfentanyl-d5 & 223.1733 & 5.91 \\
\hline ADB-PINACA-d9 & 353.2777 & 11.53 \\
\hline AM 2201 N-(4-hydroxypentyl) metabolite-d5 & 380.1948 & 10.69 \\
\hline Benocyclidine-d10 & 309.2335 & 9.27 \\
\hline Butylone-d3 & 224.1240 & 6.51 \\
\hline cis-Tramadol-d6 & 269.2262 & 7.17 \\
\hline JWH 007-d9 & 328.2501 & 12.98 \\
\hline JWH 018-d9 & 350.2345 & 12.65 \\
\hline JWH 073 5-Hydroxyindole metabolite-d7 & 350.2012 & 11.21 \\
\hline JWH 081 N-pentanoic acid metabolite-d5 & 406.1941 & 10.99 \\
\hline MAM 2201 N-pentanoic acid metabolite-d5 & 390.1992 & 11.11 \\
\hline Norsufentanil-d3 & 279.2026 & 8.05 \\
\hline PB-22-d9 & 367.2246 & 11.99 \\
\hline RCS-4 N-(5-hydroxypentyl) metabolite-d5 & 342.1992 & 10.46 \\
\hline UR-144 N-(4-hydroxypentyl) metabolite-d5 & 332.2512 & 11.38 \\
\hline XLR11-d 5 & 334.2469 & 12.10 \\
\hline
\end{tabular}




\subsubsection{LC-QTOF-MS Method}

The LC separation was conducted with a gradient with $5 \mathrm{mM}$ ammonium formate (AF) in HPLC water with $0.1 \%$ formic acid (FA) as the aqueous mobile phase (A) and $\mathrm{MeOH}$ with $0.1 \% \mathrm{FA}$ as the organic mobile phase (B). The mobile phase gradient was employed at a flow rate of $0.3 \mathrm{~mL} / \mathrm{min}$ with composition beginning at $5 \% \mathrm{~B}$ from $0-1$ min, then increasing to $95 \%$ B from $1-9.5 \mathrm{~min}$, and remaining at $9.5 \mathrm{~min}$ for the remainder of the analysis time. An equilibration time of 3 min was also incorporated into the LC method. Chromatographic column temperature was maintained at $40^{\circ} \mathrm{C}$.

Detection of the chromatographic data was completed using QTOF-MS in full scan mode. The ESI source parameters were: drying gas temperature $325^{\circ} \mathrm{C}$; drying gas flow rate $8 \mathrm{~L} / \mathrm{min}$; nebulizer $35 \mathrm{psi}$; sheath gas temperature $400^{\circ} \mathrm{C}$; sheath gas flow 12 L/min. Scanning source parameters were: VCap voltage $3500 \mathrm{~V}$; nozzle voltage $0 \mathrm{~V}$; fragmentor voltage $125 \mathrm{~V}$; skimmer voltage $65 \mathrm{~V}$.

Two reference ions, provided by the reference mass solution, were monitored with mass correction to ensure proper instrumental calibration throughout analysis; $121.0509 \mathrm{~m} / \mathrm{z}$ and $922.0098 \mathrm{~m} / \mathrm{z}$.

\subsubsection{Retention Time Collection}

Prior to validation of the method, retention data for all compounds were collected to prevent inclusion of any co-eluting compounds in the validation mixtures. Individual compounds were injected at concentrations of $1 \mu \mathrm{g} / \mathrm{mL}$ in $\mathrm{MeOH}$ at volumes of $5 \mu \mathrm{L}$. Separation was conducted over 20 min using an Agilent Zorbax Eclipse Plus $\mathrm{C}_{18}$ Rapid Resolution HD column (3.0 x $100 \mathrm{~mm} ; 1.8 \mu \mathrm{m})$ and the LC-QTOF-MS method described 
above. Retention data was collected using Full Scan mode with the MS range set to 50$1700 \mathrm{~m} / \mathrm{z}$ with an MS acquisition rate of 10 spectra per second.

The FBF function of Qualitative Analysis software was used to isolate the individual compound from each injected solution and the corresponding retention data were recorded. These data were used to design the validation mixtures so that no two components of a mixture would co-elute, thus interfering with identification and quantitation of the compounds.

\subsubsection{Preparation of Samples in Urine}

Urine samples were prepared for all aspects of validation. Calibrators were prepared in urine at seven different concentrations ranging between $2-120 \mathrm{ng} / \mathrm{mL}$ for each of the three validation mixes. Samples included blank urine diluted at a ratio of 1:5 using aqueous mobile phase. The working validation mixtures containing compounds at concentrations of $200 \mathrm{ng} / \mathrm{mL}$ in $\mathrm{MeOH}$ were spiked into the diluted urine at appropriate volumes to make the calibrators. Each sample was also spiked with the IS mixture at a concentration of $40 \mathrm{ng} / \mathrm{mL}$.

Quality control (QC) samples were created at three concentration levels within the calibration range; low, medium, and high $(5,20$, and $80 \mathrm{ng} / \mathrm{mL})$ in urine diluted $(1: 5)$ with aqueous mobile phase. The QCs were also spiked with the IS mixture at a concentration of $40 \mathrm{ng} / \mathrm{mL}$. Blank urine samples (matrix blanks) were also prepared using pooled, blank urine diluted (1:5) with aqueous mobile phase. The calibrators, QCs, and matrix blanks were used throughout the method validation process. 


\subsubsection{Preparation of Blind-Spiked Specimens}

Two sets of 20 specimens were prepared by a third party not involved in the analysis using blank, pooled urine from UTAK Laboratories. The urine samples were spiked with 0-1 NPS and diluted with aqueous mobile phase at a 1:5 dilution. No internal standard was included as this was a purely qualitative investigation.

\subsection{Results and Discussion}

\subsubsection{Linearity}

Linearity experiments were conducted at seven calibration concentrations between $2-120 \mathrm{ng} / \mathrm{mL}$. These concentrations were set at 2, 5, 10, 20, 50, 100, and 120 $\mathrm{ng} / \mathrm{mL}$ and five replicates were run for each concentration level. Measured concentrations were then modeled using linear regression and a weight of $1 / x$ was applied. An example weighted calibration curve for 25I-NBF from validation mix 2 is presented in Figure 5. An example of an unweighted calibration curve for 25I-NBF is presented in Figure 4. There were no major differences between the unweighted and weighted calibration curves, but per OSAC guidelines, a weighted model should be used when there are notable differences between variances at the lowest and highest concentrations. This is the case when the concentration range is larger than one order of magnitude, as was the case with the calibration levels chosen in this study.

Calibration curves were created for validation mixes 1, 2 and 3. Mix 1 contained 29 compounds, all of which had linear calibration curves. Mix 2 contained 33 compounds, of which 30 had linear calibration curves. Mix 3 contained 28 compounds, of which 24 had acceptable linearity based on their calibration curves. The data collected 
during the linearity studies were also used in the calculation of the limit of detection (LOD) and the limit of quantitation (LOQ) for each compound.

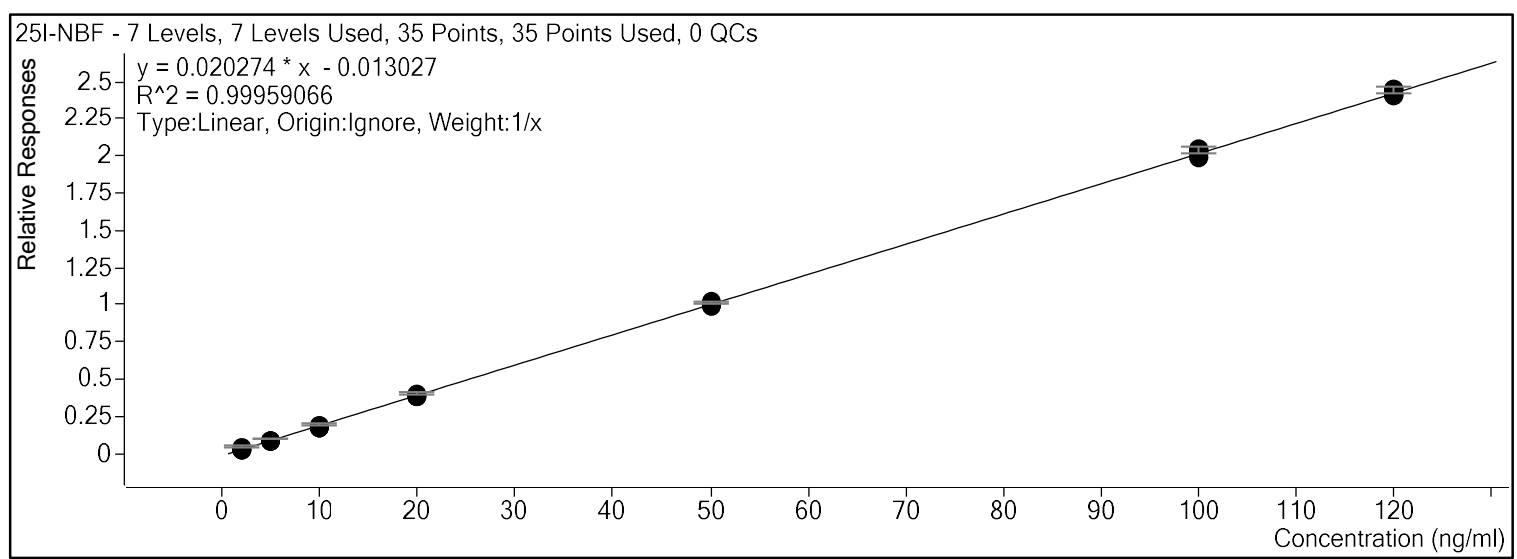

Figure 5. Weighted calibration curve for 25I-NBF from validation Mix 2.

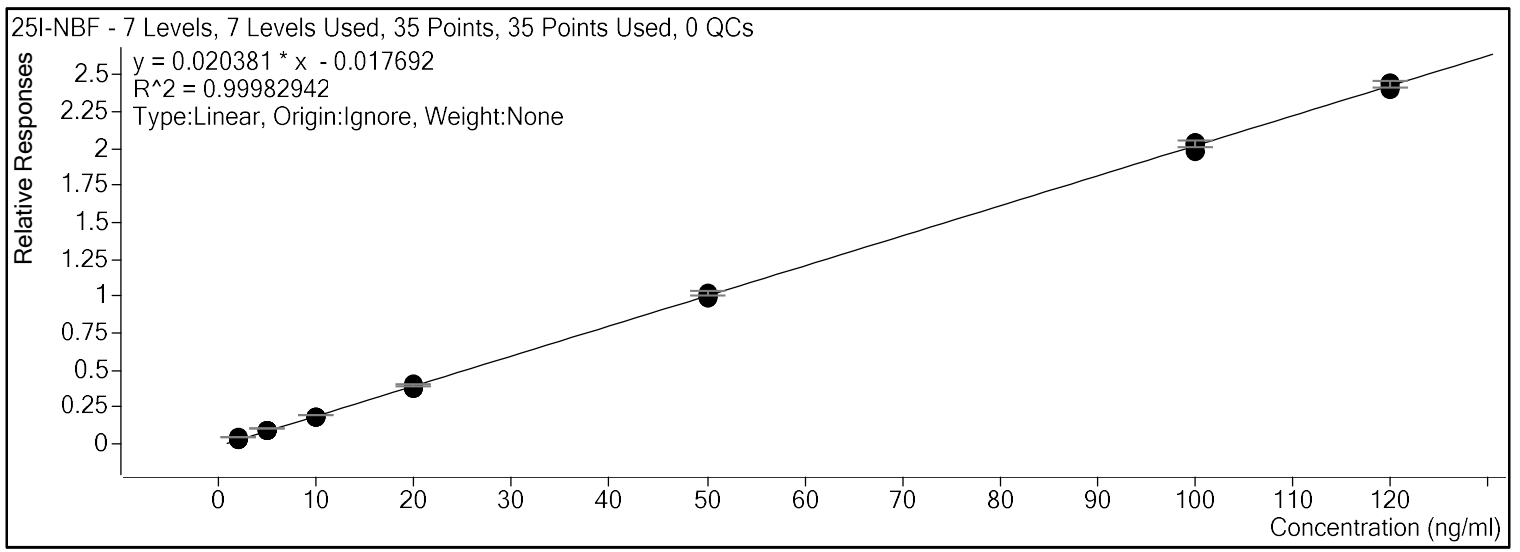

Figure 4. Unweighted calibration curve for 25I-NBF from validation Mix 2.

\subsubsection{Accuracy and Precision}

Accuracy (bias) is the determination of how closely a compound's calculated concentration corresponds to its actual concentration. Precision is a measure of how closely the calculated concentrations of a compound compare with each other within a 
single analysis and between separate analyses. Accuracy and precision studies were preformed concurrently with the same samples known as the QC samples.

Samples for the accuracy and precision studies were created at three different concentrations, representing the low, medium, and high range of the concentrations used in the calibration curves. Three replicates of each concentration level were prepared on each of five consecutive days and analyzed on the day they were prepared.

Accuracy was then calculated for each compound in each mixture using the following equation:

$$
\begin{gathered}
\text { Bias (\%) at Concentration }= \\
{\left[\frac{\text { Gran Mean of Calculated Concentration-Nomina Concentration }}{\text { Nominal Concentration }}\right] \times 100}
\end{gathered}
$$

where the calculated concentration is the measured concentration of the compound during analysis and the nominal concentration is the concentration set for that calibration level. At each concentration, the maximum acceptable bias is $\pm 20 \%$. The data used in bias studies were also used to calculate precision, as per the OSAC guidelines. ${ }^{150}$

Precision studies were conducted to determine variability between runs on the same day (intra-day variability) and between runs conducted on separate days (inter-day variability). General precision values are expressed in terms of the coefficient of variation $(\% \mathrm{CV})$ as seen in Equation 3 following the calculation of the mean and standard deviation $(s)$ of the response at each concentration.

$$
\% C V=\frac{s}{\text { mean response }}
$$


Acceptable precision values must have a $\% \mathrm{CV}$ within a range of $\pm 20 \%$ at each concentration. Calculation of within-run precision was done using data from each of the three replicates at each concentration level (Equation 4). The largest \% CV value calculated at each concentration was used to assess the within-run precision.

$$
\text { Within }- \text { Run } C V(\%)=\frac{\text { std deviation of a single run of samples }}{\text { mean calculated value of a single run of samples }} \times 100
$$

Calculation of between-run precision was conducted with data collected at each concentration over the course of five runs completed on separation days (Equation 5).

$$
\text { Between }- \text { Run CV }(\%)=\frac{\text { std dev of all observations for each concentration }}{\text { grand mean for each concentration }} \times 100
$$

Bias and precision data for Mix 1 are presented in Table 8 with all calculated bias values at each concentration within $\pm 10.1 \%$. Overall precision values at each concentration were within $\pm 10 \% \mathrm{CV}$, with all within-run and between-run precision values within $\pm 20 \%$. Most of the within- and between-run precision values were within $\pm 10 \%$ with the exception of the low concentration within-run values.

The bias and precision data were calculated for the 30 compounds in Mix 2 which demonstrated linearity. The bias and precision data are presented in Table 9 with all values at each concentration within the limits of $\pm 20 \%$. All bias values were within $\pm 10 \%$. Overall precision values were all within the limits of $\pm 20 \% \mathrm{CV}$, with the majority within $\pm 10 \%$. For the within-run and between-run precision values, all compounds had calculated values within $\pm 20 \%$. 
The bias and precision data were calculated for the 24 compounds in Mix 3 with demonstrated linearity. The bias and precision data are presented in Table 10 with all values at each concentration within the limits of $\pm 20 \%$. All bias values were within $\pm 10 \%$. Overall precision values were all within $\pm 10 \% \mathrm{CV}$. The within-run and betweenrun precision values were all within $\pm 20 \%$, with the majority within $\pm 10 \%$.

\subsubsection{Limits of Detection and Quantitation}

The limit of detection (LOD) and limit of quantitation (LOQ) represent the lowest concentrations at which an analyte may be detected by a method and quantitated, respectively. Both values were calculated using the slope $(m)$ and y-intercept values generated during the linearity studies. The LOD was calculated using the following equation:

$$
L O D=\frac{3.3 s_{y}}{A v g_{m}}
$$

where $\mathrm{s}_{y}$ is the standard deviation of the $\mathrm{y}$-intercept and $A v g_{m}$ is the average slope. The LODs for compounds in Mix 1 were all in the low $\mathrm{ng} / \mathrm{mL}(\mathrm{ppb})$ range with values ranging from 0.2 to $2.9 \mathrm{ng} / \mathrm{mL}$ (Table 11). The LODs for compounds in Mix 2 were all in the low $\mathrm{ng} / \mathrm{mL}$ (ppb) range with values ranging from 0.1 to $2.2 \mathrm{ng} / \mathrm{mL}$ (Table 12). The LODs calculated for compounds in Mix 3 were also in the lower $\mathrm{ng} / \mathrm{mL}$ range with values between 0.2 and $1.6 \mathrm{ng} / \mathrm{mL}$. Most values were below $1.1 \mathrm{ng} / \mathrm{mL}$ (Table 13).

The LOQ values were calculated in a similar manner to the LOD values using the following equation:

$$
L O Q=\frac{10 y}{A v g_{m}}
$$


All of the calculated LOQs for Mix 1 are shown in Table 11 with a range of values from 0.6 to $8.8 \mathrm{ng} / \mathrm{mL}$, with most values $\leq 5.0 \mathrm{ng} / \mathrm{mL}$. The LOQ values for Mix 2 are shown in Table 12 with a range of values from 0.3 to $6.8 \mathrm{ng} / \mathrm{mL}$, with most values at less than $5.0 \mathrm{ng} / \mathrm{mL}$. The LOQ values for Mix 3 are shown in Table 13 with values between 0.6 and $4.7 \mathrm{ng} / \mathrm{mL}$.

The calculated LOD and LOQ values demonstrate that the method is sensitive enough to detect low concentrations of analytes in human urine and can be applied to specimens with compounds present at pharmacologically relevant levels. A wide range of NPS concentrations in human specimens have been reported in the literature. For some SC, such as JWH 018, concentrations have been reported in postmortem cases between 0.1 and $199 \mathrm{ng} / \mathrm{mL}$ with an average concentration of $17.5 \mathrm{ng} / \mathrm{mL},{ }^{151}$ which is within the range of detection of the method presented in this research for related JWH 018 compounds whose LOD and LOQ values were as low as 0.4 and $1.1 \mathrm{ng} / \mathrm{mL}$, respectively. Another case reported in the literature involved acute fatal poisoning by NNEI, an analog of JWH-018. The concentrations in whole blood collected postmortem ranged from $0.64-0.99 \mathrm{ng} / \mathrm{mL}$ depending on from where in the body the samples were collected. ${ }^{152}$ The method validated in the present work achieved LOD and LOQ values for 5-fluoro NNEI of 0.2 and $0.6 \mathrm{ng} / \mathrm{mL}$, respectively, indicating that the method would be suitable for use in detecting NNEI and related analogs in real-world samples. A third case of NPS in postmortem samples was from a fatal case of multiple drug intoxication caused by the SCs AB-CHMINACA and 5-fluoro-AMB, combined with diphenidine. Postmortem tissue concentrations for AB-CHMINACA ranged from 7.55-38.9 ng/g. ${ }^{153}$ The validated method obtained LOD and LOQ values of 0.8 and $2.3 \mathrm{ng} / \mathrm{mL}$, respectively, 
for AB-CHMINACA. The values reported in the literature demonstrate that the LOD and LOQ values obtained by the method from the present research are relevant for use in detection of NPS in both antemortem and postmortem human specimens. 
Table 8. Bias and precision data for compounds in Mix 1. Concentrations for low, med, and high are 5, 20, and 80 ng/mL, respectively.

\begin{tabular}{|c|c|c|c|c|c|c|c|c|c|c|c|c|}
\hline & \multicolumn{3}{|c|}{$\operatorname{Bias}(\%)$} & \multicolumn{3}{|c|}{ Precision (\% CV) } & \multicolumn{3}{|c|}{ Within-Run (\%) } & \multicolumn{3}{|c|}{ Between-Run (\%) } \\
\hline Compound Name & low & med & high & low & med & high & low & med & high & low & med & high \\
\hline $\begin{array}{l}\alpha \text {-Pyrrolidinopentiophenone } \\
\text { metabolite } 1\end{array}$ & 1.6 & 2.0 & 0.4 & 6.4 & 3.2 & 0.7 & 12.9 & 4.0 & 1.3 & 6.4 & 3.2 & 0.7 \\
\hline 25I-NBMD & 1.6 & 2.0 & 0.4 & 5.6 & 3.1 & 0.7 & 10.0 & 4.6 & 1.3 & 5.6 & 3.1 & 0.7 \\
\hline 3-Methylbuphedrone & 1.7 & 2.1 & 0.4 & 3.5 & 2.6 & 0.7 & 6.5 & 4.8 & 0.7 & 3.5 & 2.6 & 0.7 \\
\hline 4-APDB & 0.8 & 1.0 & 0.2 & 3.6 & 2.5 & 0.9 & 7.8 & 3.6 & 2.0 & 3.6 & 2.5 & 0.9 \\
\hline $\begin{array}{c}\text { 4-fluoro- } \alpha \text { - } \\
\text { Pyrrolidinobutiophenone }\end{array}$ & 1.4 & 1.8 & 0.4 & 4.4 & 3.7 & 1.0 & 5.1 & 3.6 & 1.7 & 4.4 & 3.7 & 1.0 \\
\hline $\begin{array}{c}\text { 4'-fluoro- } \alpha \text { - } \\
\text { Pyrrolidinopropiophenone }\end{array}$ & 1.6 & 2.0 & 0.4 & 5.1 & 4.0 & 0.9 & 9.3 & 2.0 & 1.4 & 5.1 & 4.0 & 0.9 \\
\hline 4-hydroxy MET & 2.8 & 3.2 & 0.6 & 5.0 & 3.1 & 0.7 & 10.2 & 3.0 & 1.1 & 5.0 & 3.1 & 0.7 \\
\hline 4-methoxy PV8 & 1.2 & 1.5 & 0.3 & 4.5 & 2.3 & 0.8 & 9.0 & 4.3 & 1.7 & 4.5 & 2.3 & 0.8 \\
\hline $\begin{array}{c}\text { 4-methoxy- } \alpha- \\
\text { Pyrrolidinopentiophenone }\end{array}$ & 1.9 & 2.4 & 0.5 & 5.5 & 3.0 & 0.8 & 12.0 & 3.5 & 1.7 & 5.5 & 3.0 & 0.8 \\
\hline $\begin{array}{c}\text { 4-Methyl- } \alpha- \\
\text { ethylaminobutiophenone }\end{array}$ & 3.3 & 4.2 & 0.8 & 3.5 & 2.7 & 1.0 & 5.6 & 3.9 & 1.4 & 3.5 & 2.7 & 1.0 \\
\hline 5-fluoro SDB-006 & 2.2 & 2.7 & 0.5 & 8.4 & 4.6 & 1.1 & 15.5 & 3.8 & 1.4 & 8.4 & 4.6 & 1.1 \\
\hline 5-Fluoropentylindole & 3.4 & 4.2 & 0.8 & 5.3 & 2.5 & 0.9 & 11.1 & 3.6 & 2.1 & 5.3 & 2.5 & 0.9 \\
\hline 5-MAPB & 1.2 & 1.5 & 0.3 & 6.5 & 3.0 & 1.1 & 12.0 & 6.1 & 2.5 & 6.5 & 3.0 & 1.1 \\
\hline AB-005 & 0.5 & 0.6 & 0.1 & 6.7 & 3.2 & 1.4 & 10.9 & 6.0 & 3.5 & 6.7 & 3.2 & 1.4 \\
\hline AM-2233 azepane isomer & 2.3 & 2.8 & 0.6 & 7.6 & 5.2 & 1.0 & 11.8 & 9.4 & 1.6 & 7.6 & 5.2 & 1.0 \\
\hline $\begin{array}{l}\text { AM694 N-(5-hydroxypentyl) } \\
\text { metabolite }\end{array}$ & 8.1 & 10.1 & 2.0 & 5.0 & 5.5 & 1.5 & 6.4 & 7.7 & 3.1 & 5.0 & 5.5 & 1.5 \\
\hline AMT & 0.3 & 1.0 & 0.2 & 4.3 & 2.8 & 0.6 & 6.0 & 2.7 & 0.8 & 4.3 & 2.8 & 0.6 \\
\hline Benocyclidine & 3.9 & 4.8 & 1.0 & 5.3 & 4.4 & 0.8 & 7.0 & 4.0 & 0.7 & 5.3 & 4.4 & 0.8 \\
\hline Flubromazepam & 1.7 & 2.1 & 0.4 & 5.1 & 4.2 & 1.1 & 9.2 & 5.9 & 2.1 & 5.1 & 4.2 & 1.1 \\
\hline JWH 031 2'-isomer & 1.0 & 1.2 & 0.2 & 7.3 & 4.9 & 1.2 & 11.4 & 7.6 & 2.5 & 7.3 & 4.9 & 1.2 \\
\hline
\end{tabular}




\begin{tabular}{|c|c|c|c|c|c|c|c|c|c|c|c|c|}
\hline $\begin{array}{c}\text { JWH 081 N-(5- } \\
\text { hydroxypentyl) metabolite }\end{array}$ & 1.2 & 1.5 & 0.3 & 7.0 & 4.7 & 0.6 & 11.4 & 10.7 & 1.1 & 7.0 & 4.7 & 0.6 \\
\hline $\begin{array}{c}\text { JWH 200 5-hydroxyindole } \\
\text { metabolite }\end{array}$ & 1.5 & 1.9 & 0.4 & 8.8 & 3.4 & 1.5 & 18.1 & 4.8 & 3.2 & 8.8 & 3.4 & 1.5 \\
\hline JWH 203 & 1.0 & 1.3 & 0.3 & 7.6 & 4.3 & 0.7 & 13.2 & 8.2 & 1.3 & 7.6 & 4.3 & 0.7 \\
\hline N-Methyltryptamine & 0.01 & 0.01 & 0.003 & 4.8 & 2.4 & 0.7 & 6.9 & 4.1 & 1.5 & 4.8 & 2.4 & 0.7 \\
\hline NPB-22 & 1.0 & 1.3 & 0.3 & 6.5 & 4.1 & 1.7 & 10.5 & 5.5 & 3.9 & 6.5 & 4.1 & 1.7 \\
\hline $\begin{array}{c}\text { PB-22 6-hydroxyisoquinoline } \\
\text { isomer }\end{array}$ & 0.04 & 0.05 & 0.009 & 7.6 & 3.9 & 1.0 & 11.1 & 5.2 & 2.1 & 7.6 & 3.9 & 1.0 \\
\hline PCMPA & 3.7 & 4.7 & 0.9 & 7.4 & 3.3 & 0.5 & 16.4 & 4.2 & 0.6 & 7.4 & 3.3 & 0.5 \\
\hline THCA-A & 2.7 & 3.1 & 0.6 & 7.2 & 4.8 & 0.8 & 15.3 & 7.6 & 1.1 & 7.2 & 4.8 & 0.8 \\
\hline UR-144 N-heptyl analog & 1.8 & 2.2 & 0.4 & 6.1 & 5.5 & 1.0 & 7.9 & 5.8 & 0.7 & 6.1 & 5.5 & 1.0 \\
\hline
\end{tabular}


Table 9. Bias and precision data for compounds in Mix 2. Concentrations for low, med, and high are 5, 20, and 80 ng/mL, respectively.

\begin{tabular}{|c|c|c|c|c|c|c|c|c|c|c|c|c|}
\hline & \multicolumn{3}{|c|}{ Bias (\%) } & \multicolumn{3}{|c|}{ Precision ( $\% \mathrm{CV})$} & \multicolumn{3}{|c|}{ Within-Run (\%) } & \multicolumn{3}{|c|}{ Between-Run (\%) } \\
\hline Compound Name & low & med & high & low & med & high & low & med & high & low & med & high \\
\hline $\begin{array}{c}\text { 2,3-methylenedoxy } \\
\text { pyrovalerone }\end{array}$ & 2.7 & 0.6 & 0.3 & 7.7 & 2.6 & 1.5 & 17.0 & 2.9 & 2.5 & 7.7 & 2.6 & 1.5 \\
\hline $25 \mathrm{I}-\mathrm{NBF}$ & 1.8 & 1.8 & 0.7 & 3.5 & 3.0 & 2.5 & 6.6 & 5.1 & 2.6 & 3.5 & 3.0 & 2.5 \\
\hline 2C-T-2 & 1.3 & 1.0 & 3.2 & 6.2 & 4.4 & 6.9 & 11.0 & 4.3 & 1.9 & 6.2 & 4.4 & 6.9 \\
\hline $2 \mathrm{C}-\mathrm{T}-4$ & 3.0 & 4.4 & 2.7 & 9.7 & 6.6 & 7.4 & 6.7 & 6.1 & 2.8 & 9.7 & 6.6 & 7.4 \\
\hline 2-fluoromethcathinone & 1.8 & 3.0 & 1.7 & 9.2 & 6.4 & 4.3 & 10.1 & 9.1 & 4.4 & 9.2 & 6.4 & 4.3 \\
\hline $\begin{array}{l}\text { 3,4-dimethoxy- } \alpha \text { - } \\
\text { pyrrolidinopentiophenone }\end{array}$ & 0.5 & 1.5 & 1.3 & 5.6 & 5.6 & 4.5 & 8.7 & 8.4 & 3.8 & 5.6 & 5.6 & 4.5 \\
\hline $\begin{array}{c}\text { 3-methyl- } \alpha \text { - } \\
\text { pyrrolidinopropiophenone }\end{array}$ & 4.1 & 0.9 & 3.1 & 5.6 & 8.7 & 5.7 & 6.9 & 7.0 & 3.0 & 5.6 & 8.7 & 5.7 \\
\hline $\begin{array}{c}\text { 4'-methyl-N- } \\
\text { methylhexanophenone }\end{array}$ & 2.5 & 0.1 & 0.5 & 5.3 & 5.4 & 2.0 & 9.3 & 7.5 & 2.6 & 5.3 & 5.4 & 2.0 \\
\hline $\begin{array}{c}\text { 4-ethyl-N,N- } \\
\text { dimethylcathinone }\end{array}$ & 3.6 & 0.8 & 0.6 & 5.8 & 5.2 & 3.0 & 7.6 & 6.9 & 3.2 & 5.8 & 5.2 & 3.0 \\
\hline 4-hydroxy MiPT & 5.1 & 0.5 & 3.7 & 5.5 & 6.8 & 6.8 & 5.8 & 2.6 & 1.5 & 5.5 & 6.8 & 6.8 \\
\hline 4-MMC & 1.5 & 1.7 & 2.7 & 6.3 & 8.6 & 5.2 & 8.7 & 6.8 & 3.2 & 6.3 & 5.6 & 5.2 \\
\hline A-796260 & 4.5 & 5.8 & 2.3 & 9.9 & 5.7 & 6.4 & 13.6 & 3.0 & 1.4 & 9.9 & 5.7 & 6.4 \\
\hline AB-005 azepine isomer & 4.1 & 0.8 & 2.8 & 5.0 & 6.0 & 4.8 & 8.3 & 3.3 & 3.9 & 5.0 & 6.0 & 4.8 \\
\hline $\begin{array}{l}\text { AB-FUBINACA 3- } \\
\text { fluorobenzyl isomer }\end{array}$ & 4.0 & 1.3 & 3.3 & 5.6 & 5.1 & 6.0 & 8.4 & 4.7 & 4.0 & 5.6 & 5.1 & 6.0 \\
\hline ADB-PINACA isomer 1 & 4.0 & 3.7 & 0.6 & 6.3 & 1.8 & 3.2 & 11.0 & 2.9 & 4.6 & 6.3 & 1.8 & 3.2 \\
\hline $\begin{array}{c}\text { AKB48 N-(5-fluoropentyl) } \\
\text { analog }\end{array}$ & 3.5 & 2.0 & 0.7 & 6.5 & 5.5 & 2.0 & 6.4 & 2.9 & 2.5 & 6.5 & 5.5 & 2.0 \\
\hline Clencyclohexerol & 6.1 & 3.4 & 0.4 & 15.7 & 1.6 & 1.1 & 9.6 & 2.3 & 1.7 & 15.7 & 1.6 & 1.1 \\
\hline EG-018 & 5.2 & 7.0 & 0.8 & 6.9 & 6.0 & 3.2 & 3.7 & 2.5 & 3.6 & 6.9 & 6.0 & 3.2 \\
\hline
\end{tabular}




\begin{tabular}{|c|c|c|c|c|c|c|c|c|c|c|c|c|}
\hline $\begin{array}{c}\text { JWH } 018 \mathrm{~N}-(5 \text {-hydroxypentyl) } \\
\text { metabolite }\end{array}$ & 4.1 & 0.9 & 2.5 & 5.6 & 8.0 & 4.0 & 6.0 & 2.9 & 1.4 & 5.6 & 8.0 & 4.0 \\
\hline $\begin{array}{c}\text { JWH } 018 \text { N-propanoic acid } \\
\text { metabolite }\end{array}$ & 0.6 & 0.2 & 3.3 & 5.4 & 4.8 & 6.4 & 11.5 & 4.7 & 3.4 & 5.4 & 4.8 & 6.4 \\
\hline KM 233 & 2.6 & 9.8 & 2.4 & 11.4 & 7.2 & 7.5 & 4.3 & 2.8 & 4.1 & 11.4 & 7.2 & 7.5 \\
\hline Loperamide & 0.9 & 2.9 & 0.01 & 3.9 & 3.0 & 1.50 & 5.5 & 4.2 & 2.2 & 3.9 & 3.0 & 1.5 \\
\hline $\begin{array}{l}\text { MAM2201 N-pentanoic acid } \\
\text { metabolite }\end{array}$ & 1.5 & 3.7 & 1.5 & 6.0 & 2.6 & 2.2 & 12.1 & 3.6 & 2.3 & 6.0 & 2.6 & 2.2 \\
\hline N-Ethylbuphedrone & 2.5 & 2.3 & 0.8 & 8.1 & 8.1 & 4.2 & 4.3 & 6.5 & 7.2 & 8.1 & 8.1 & 4.2 \\
\hline $\mathrm{PCPr}$ & 5.6 & 0.001 & 3.7 & 6.9 & 7.0 & 6.5 & 8.5 & 10.4 & 8 & 6.9 & 7.0 & 6.5 \\
\hline RCS-4 2-methoxy isomer & 7.5 & 3.6 & 1.5 & 6.2 & 4.9 & 1.2 & 7.4 & 3.0 & 1.6 & 6.2 & 4.9 & 1.2 \\
\hline SER-601 & 3.5 & 3.3 & 2.3 & 4.6 & 2.5 & 5.0 & 6.6 & 2.2 & 1.5 & 4.6 & 2.5 & 5.0 \\
\hline UR-144 Degradant & 6.9 & 4.1 & 1.4 & 5.4 & 1.8 & 4.0 & 8.7 & 2.7 & 1.5 & 5.4 & 1.8 & 4.0 \\
\hline $\begin{array}{l}\text { XLR11 N-(2-fluoropentyl) } \\
\text { isomer }\end{array}$ & 7.3 & 2.4 & 0.3 & 7.5 & 4.0 & 2.2 & 8.5 & 2.7 & 0.7 & 7.5 & 4.0 & 2.2 \\
\hline$\triangle 8$-THC & 6.1 & 6.2 & 0.5 & 10.3 & 6.3 & 3.5 & 10.6 & 5.2 & 4.4 & 10.3 & 6.3 & 3.5 \\
\hline
\end{tabular}


Table 10. Bias and precision data for compounds in Mix 3. Concentrations for low, med, and high are 5, 20, and $80 \mathrm{ng} / \mathrm{mL}$, respectively.

\begin{tabular}{|c|c|c|c|c|c|c|c|c|c|c|c|c|}
\hline \multirow[b]{2}{*}{ Compound Name } & \multicolumn{3}{|c|}{ Bias (\%) } & \multicolumn{3}{|c|}{ Precision (\% CV) } & \multicolumn{3}{|c|}{ Within-Run (\%) } & \multicolumn{3}{|c|}{ Between-Run (\%) } \\
\hline & low & med & high & low & med & high & low & med & high & low & med & high \\
\hline (R)-(-)-MT-45 & 0.6 & 0.7 & 0.2 & 1.3 & 1.4 & 0.5 & 1.9 & 1.4 & 0.7 & 1.3 & 1.4 & 0.5 \\
\hline $\begin{array}{c}2,3- \\
\text { Dichlorophenylpiperazine }\end{array}$ & 3.3 & 4.1 & 0.8 & 3.5 & 4.5 & 0.9 & 2.6 & 2.5 & 0.4 & 3.5 & 4.5 & 0.9 \\
\hline 25H-NBOMe & 1.0 & 1.2 & 0.3 & 1.2 & 1.0 & 0.6 & 2.1 & 1.0 & 1.1 & 1.2 & 1.0 & 0.6 \\
\hline $2 \mathrm{C}-\mathrm{T}$ & 0.4 & 0.5 & 0.1 & 1.4 & 1.3 & 0.5 & 2.6 & 0.9 & 0.8 & 1.4 & 1.3 & 0.5 \\
\hline 2-Methoxyamphetamine & 0.5 & 0.7 & 0.2 & 1.1 & 1.3 & 0.6 & 0.8 & 0.7 & 0.9 & 1.1 & 1.2 & 0.6 \\
\hline 3,4-Dimethylethcathinone & 0.5 & 0.7 & 0.1 & 0.9 & 1.1 & 0.5 & 0.9 & 1.9 & 0.7 & 0.9 & 1.1 & 0.5 \\
\hline $3 \mathrm{C}-\mathrm{P}$ & 0.3 & 0.3 & 0.1 & 1.5 & 1.4 & 0.5 & 2.7 & 1.2 & 0.9 & 1.5 & 1.4 & 0.5 \\
\hline 4-Methoxyamphetamine & 0.6 & 0.8 & 0.2 & 1.6 & 1.0 & 0.5 & 3.7 & 0.7 & 0.8 & 1.6 & 1.0 & 0.5 \\
\hline 5-fluoro NNEI & 2.1 & 2.6 & 0.5 & 2.6 & 3.1 & 0.7 & 1.4 & 1.3 & 0.7 & 2.6 & 3.1 & 0.7 \\
\hline $\begin{array}{c}\text { 9- } \\
\text { octadecenamide/oleamide }\end{array}$ & 0.2 & 0.2 & 0.04 & 1.7 & 2.4 & 0.8 & 2.2 & 4.3 & 1.1 & 1.7 & 2.4 & 0.8 \\
\hline AB-CHMINACA & 0.4 & 0.5 & 0.1 & 0.7 & 0.7 & 0.5 & 1.1 & 0.7 & 0.8 & 0.7 & 0.7 & 0.5 \\
\hline $\begin{array}{l}\text { AKB48 N-pentanoic acid } \\
\text { metabolite }\end{array}$ & 1.0 & 1.3 & 0.3 & 1.0 & 0.9 & 0.4 & 1.3 & 0.9 & 0.6 & 1.0 & 0.9 & 0.4 \\
\hline Cathine & 0.4 & 2.2 & 0.5 & 7.3 & 4.4 & 1.3 & 11.6 & 5.8 & 2.0 & 7.3 & 4.4 & 1.3 \\
\hline Diclofensine & 0.2 & 0.2 & 0.04 & 0.5 & 0.6 & 0.5 & 0.7 & 0.8 & 0.8 & 0.5 & 0.6 & 0.5 \\
\hline FUB-PB-22 & 1.3 & 1.6 & 0.3 & 1.2 & 1.5 & 0.6 & 1.5 & 1.6 & 1.0 & 1.2 & 1.5 & 0.6 \\
\hline HMA & 1.1 & 0.3 & 0.01 & 4.4 & 1.7 & 0.7 & 10.2 & 3.8 & 1.1 & 4.4 & 1.7 & 0.7 \\
\hline MBZP & 5.3 & 1.6 & 0.1 & 5.1 & 2.1 & 0.3 & 2.6 & 2.6 & 0.4 & 5.1 & 2.1 & 0.3 \\
\hline Mephedrone & 0.4 & 0.4 & 0.1 & 1.0 & 1.2 & 0.5 & 1.1 & 1.9 & 0.7 & 1.0 & 1.2 & 0.5 \\
\hline $\begin{array}{c}\text { Methylenedioxy } \\
\text { Pyrovalerone metabolite } 2\end{array}$ & 0.5 & 0.6 & 0.1 & 1.3 & 0.9 & 0.5 & 2.4 & 1.2 & 1.0 & 1.3 & 0.9 & 0.5 \\
\hline N-methyl-2-AI & 0.7 & 0.9 & 0.2 & 1.3 & 1.3 & 0.5 & 1.9 & 1.5 & 0.8 & 1.3 & 1.3 & 0.5 \\
\hline NRG-3 & 0.03 & 0.04 & 0.01 & 0.7 & 0.9 & 0.7 & 0.8 & 1.3 & 1.1 & 0.7 & 0.9 & 0.7 \\
\hline
\end{tabular}




\begin{tabular}{|c|c|c|c|c|c|c|c|c|c|c|c|c|}
\hline $\begin{array}{c}\text { PB-22 N-(5- } \\
\text { hydroxypentyl) metabolite }\end{array}$ & 1.0 & 1.3 & 0.3 & 1.8 & 1.6 & 0.5 & 3.2 & 0.9 & 0.7 & 1.8 & 1.6 & 0.5 \\
\hline $\begin{array}{c}\text { RCS-4 N-(4- } \\
\text { hydroxypentyl) metabolite }\end{array}$ & 0.5 & 0.7 & 0.1 & 1.7 & 1.1 & 0.6 & 3.6 & 1.0 & 1.1 & 1.7 & 1.1 & 0.6 \\
\hline $\begin{array}{c}\text { UR-144 N-(2-chloropentyl) } \\
\text { analog }\end{array}$ & 0.03 & 0.03 & 0.01 & 0.8 & 0.4 & 0.3 & 1.7 & 0.5 & 0.4 & 0.8 & 0.4 & 0.3 \\
\hline
\end{tabular}




\subsubsection{Freeze-Thaw Stability}

Storage conditions can affect analyte stability in samples and in forensic toxicology it is common for laboratories to freeze urine specimens upon receipt to preserve them until a time when they may be analyzed. Thus, analyte stability must be determined using a series of freeze and thaw cycles. In accordance with OSAC method validation guidelines, urine samples fortified with the analytes underwent freeze-thaw cycles in which they were frozen for 24 hours then removed from the freezer and allowed to thaw unassisted at room temperature. This cycle was then repeated two more times. An analyte was considered stable when the average signal was within the method's acceptable bias, which in these studies was $\pm 20 \%$ of the time zero average signal.

Samples in this series of studies were prepared in four sets of blank urine diluted with water (1:5) with Mixes 2 and 3 at high and low calibration concentrations (e.g., 5 and $120 \mathrm{ng} / \mathrm{mL}$ ). These sets were labeled as $\mathrm{T}_{0}, \mathrm{~T}_{1}, \mathrm{~T}_{2}$, and $\mathrm{T}_{3}$, representing each time point of the three freeze-thaw cycles, with $\mathrm{T}_{0}$ as the time zero signal where the sample has not been subjected to any freeze-thaw cycles. The analyte signal was determined using the analyte response determined using the Quantitative Analysis software. The averages of these values at each concentration from each time point were then compared to the corresponding time zero value average using Equation 2. Results for Mix 2 after three freeze-thaw cycles are displayed in Table 14 and the results for Mix 3 are displayed in Table 15.

The majority of the analytes in Mix 2 fell within $\pm 20 \%$ bias with only a few compounds at the low concentration having a bias value greater than $20 \%$. The seven compounds in Mix 2 at with significant stability problems at the low concentration after 
three freeze-thaw cycles were all synthetic cannabinoids. Stability of synthetic cannabinoids has not been extensively studied, although there have been reports of instability for several compounds. ${ }^{26,154}$

All compounds in Mix 3 at the high concentration had bias values within $\pm 20 \%$ after three freeze-thaw cycles. At the low concentration, only two compounds had bias values $>20 \%$ : oleamide and AKB48 N-pentanoic acid metabolite. Oleamide is an agonist of cannabinoid $1\left(\mathrm{CB}_{1}\right)$ receptors and AKB48 N-pentanoic acid metabolite is a potential urinary metabolite of the synthetic cannabinoid AKB48.

\subsubsection{Matrix Effects}

Matrix effects occur when compounds that naturally occur in the sample matrix co-elute with analytes of interest and cause either suppression or enhancement of the ionization of the target molecule. Suppression or enhancement of analyte ions can affect the values for LOD, LOQ, and bias in quantitative methods and therefore, values should not exceed $\pm 25 \%$. The equation used to calculate matrix effects is shown in Equation 8 . However, if the calculated value for matrix effects is outside of the $\pm 25 \%$ range, the laboratory conducting the validation must demonstrate that the matrix effects do not cause significant adverse effects to the critical validation parameters.

$$
\text { M.E. }(\%)=\left(\frac{\bar{X} \text { area of } \text { Set } 2}{\bar{X} \text { area of Set } 1}-1\right) \times 100
$$

where $\bar{X}$ is the average area of each set, with Set 1 representing standards prepared in water and Set 2 representing standards prepared in urine. 
Samples for each set were prepared at low and high concentrations of 5 and 100 $\mathrm{ng} / \mathrm{mL}$, respectively. Six replicates of each sample at each concentration were analyzed with the peak areas of each analyte measured and recorded using the Qualitative Analysis software. Calculated ion suppression and enhancement values for Mixes 1-3 are shown in Table 16, Table 17, and Table 18, respectively.

Nearly half of the compounds in Mix 1 did not have significant ion suppression or enhancement due to matrix effects. However, there were several compounds that did exhibit significant matrix effects. More than half of the compounds in Mix 2 displayed significant ion suppression, as indicated by ionization differences between the neat samples prepared in water and the samples prepared in urine, particularly at the high concentration of $100 \mathrm{ng} / \mathrm{mL}$. Several compounds in Mix 3 also displayed significant ion suppression at both the low and high concentrations. However, the critical validation parameters (e.g., LOD, LOQ, and bias) of the compounds of both mixes prepared in urine are still well within acceptable ranges.

The matrix effects exhibited were recorded but do not prevent validation of the method for these compounds. The results of the matrix effect studies indicate that more thorough sample preparation for urine specimens containing NPS may be necessary prior to analysis. Possible cleanup steps may include further dilution (1:10) or SPE, either online or off-line. There was no clear trend as to which compounds exhibited the most significant matrix effects, however, it was noted that several of the tryptamine and metabolites of the synthetic cannabinoid classes were among those that exhibited the most ion suppression or enhancement. 


\subsubsection{Carryover}

Analytical methods that include a chromatographic separation step may be subject to issues of carryover, which occurs when analytes from a previous sample appear in the next sample of a batch, usually as a result of incomplete elution from the separation column. Carryover may lead to inaccurate qualitative or quantitative results thus negatively affecting the reliability of the method. In order to assess carryover, blank urine samples were analyzed immediately after the highest concentration calibrator, which was $120 \mathrm{ng} / \mathrm{mL}$. The analysis of the blank urine samples was conducted in triplicate for Mixes 1-3. Using both the Qualitative and Quantitative Analysis software, it was determined that there was no significant carryover of any compounds in any of the mixes. An example of the lack of carryover from the method is shown in Figure 7 through the overlay of the chromatograms generated for oleamide at $5 \mathrm{ng} / \mathrm{mL}$ and in the blank urine that was analyzed immediately following a $120 \mathrm{ng} / \mathrm{mL}$ sample. Oleamide was one of the few analytes that exhibited carryover effects and had the largest peak area of those generated. However, when compared with the peak area of oleamide demonstrated at a low concentration of $5 \mathrm{ng} / \mathrm{mL}$, it is clearly shown that the degree of carryover is minimal.

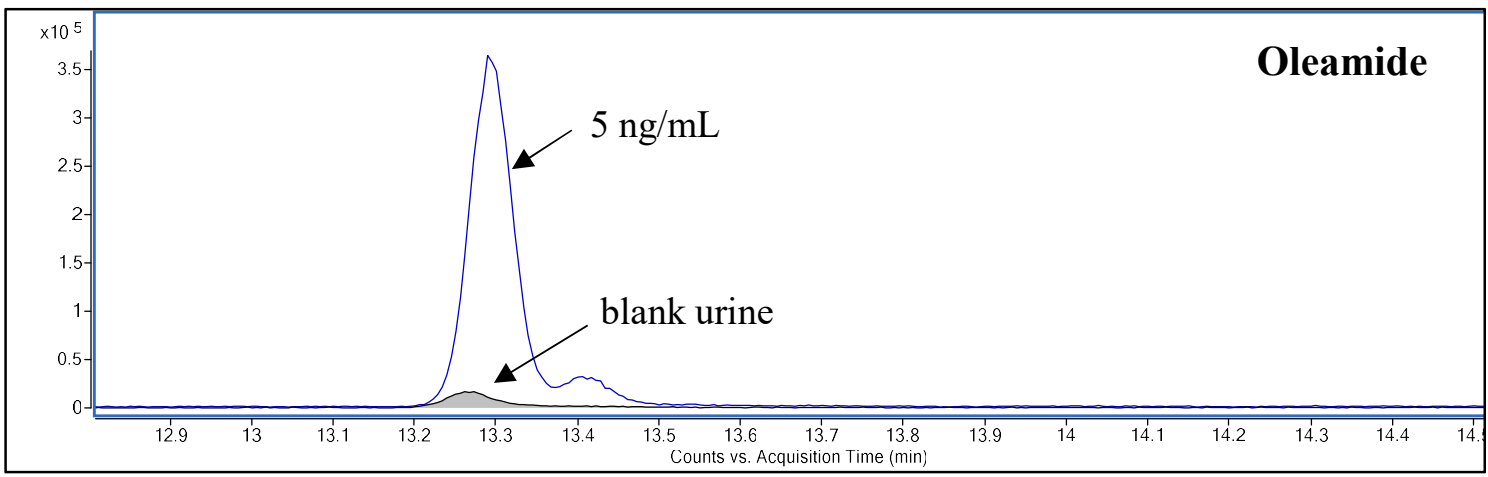

Figure 7. Carryover study for oleamide in Mix 3 comparing analyte concentration present in a $5 \mathrm{ng} / \mathrm{mL}$ sample with that in a blank urine sample that was analyzed immediately following a $120 \mathrm{ng} / \mathrm{mL}$ sample. 


\subsubsection{Analysis of Blind-Spiked Specimens}

Analysis of the blind-spiked urine specimens was conducted using the validated LC-QTOF-MS screening method. Chromatographic separation of the urine specimens was conducted using the Zorbax Eclipse Plus $\mathrm{C}_{18}$ RRHD column and an injection volume of $10 \mu \mathrm{L}$. The ESI source was operated in positive mode.

Two acquisition methods were used with the QTOF-MS; Full Scan MS and Auto MS/MS. The same LC, ESI source, and scan parameters were used for each acquisition method. Full Scan MS collected all ion data and did not subject the ions to any collision energy. MS range was $100-1000 \mathrm{~m} / \mathrm{z}$ and the acquisition rate was $1.5 \mathrm{spectra} / \mathrm{s}$. The Auto MS/MS used an MS range of 100-1000 m/z with an acquisition rate of $3 \mathrm{spectra} / \mathrm{s}$, while the MS/MS mass range was 50-1000 m/z with an acquisition rate of 3 spectra/s. MS/MS fragmentation was performed at three collision energies of 10, 20, and $40 \mathrm{eV}$. The quadrupole used a narrow isolation width of $1.3 \mathrm{~m} / \mathrm{z}$. Reference mass correction was also enabled to ensure proper instrumental calibration throughout the analysis, using masses $121.0509 \mathrm{~m} / \mathrm{z}$ and $922.0098 \mathrm{~m} / \mathrm{z}$.

Agilent MassHunter Qualitative Analysis software was used to perform library and database searches for data collected using both Full Scan MS and Auto MS/MS modes. The compound database and library previously created was used for searching. For Full Scan MS data analysis, searching was conducted using the "Find Compounds by Formula" (FBF) followed by the "Search Library" and "Search Database" commands. Analysis of the data collected using Auto MS/MS mode was very similar except that the "Find Compounds by Auto MS/MS" command was used instead of the FBF. The potential compound list generated after this command usually included over 250 
compounds per sample. This list was then subjected to a search using the accurate mass library which employed both forward (matching peaks in the sample against those in the library) and reverse (matching peaks in the library against those in the sample) searching. Search parameters were set to include precursor ion expansion of $\pm 10 \mathrm{ppm}+2 \mathrm{mDa}$ and product ion expansion of $\pm 20 \mathrm{ppm}+2 \mathrm{mDa}$. The library search parameters were set to a minimum of 70 for both forward and reverse scores.

Compounds identified by the library search were then subjected to a database search which compared the mass of a compound in the sample with the corresponding molecular formula in the database. The database search had a mass tolerance set to \pm 10 ppm. Positive identifications required a library score $>80$ and a database score $>70$. Mass error was also required to be $<10 \mathrm{ppm}$.

The scores generated through database and library searching are calculated using an algorithm that is proprietary information of the MassHunter Qualitative Analysis software. However, some factors that contribute to this score may include isotope abundance, and the presence and intensity of specific ion fragments.

Two sets of 20 blind-spiked specimens were prepared in diluted urine (1:5) by a laboratory member that was not conducting the analysis, hence the term "blind." The specimens contained 0-1 NPS and were identical for both sets. The concentration of compounds in Set 1 was either 2 or $20 \mathrm{ng} / \mathrm{mL}$ with all specimens in Set 2 prepared at a concentration of $200 \mathrm{ng} / \mathrm{mL}$.

Screening of Set 1 resulted in positive identification of the NPS or blank specimen for 13/20 specimens (Table 19). Screening of the higher level Set 2 samples resulted in more positive identifications, with 15/20 correctly identified (Table 20). 
Three of the specimens in Set 2 (specimens 4, 8, and 9) were not originally identified correctly, however, the true identity of the NPS in the specimen was included on a "hit list" or list of possible identifications. Closer analysis of the ion fragmentation patterns for the analyzed specimens, as well as consideration of the retention times included as part of the in-house database, ultimately resulted in the correct identification.

Specimen 1 from both sets contained mephedrone but was identified as 3methylmethcathinone (3-MMC) using the compound database. Both NPS have the chemical formula $\mathrm{C}_{11} \mathrm{H}_{15} \mathrm{NO}$ with an identical accurate mass of $177.1154 \mathrm{Da}$. This result demonstrates the importance of MS/MS spectral data in the identification of NPS and the determination between two very different compounds that share the same chemical formula. The MS/MS spectral data for mephedrone were not included in the HRMS library; inclusion of MS/MS data for mephedrone would have resulted in the correct identification of the compound in specimen 1.

Although not all of the compounds in the blind-spiked specimens were correctly identified, the preliminary qualitative screening results indicate the utility of the compound database and HRMS spectral library for identification of NPS in human urine specimens. It is important to note that the HRMS library used in the above study was created in-house and had not been curated to remove background ions as curation of the library was not included in the scope of the present research. The method used for screening did not include any extraction or preconcentration procedures prior to analysis, and instead utilized a "dilute-and-shoot" approach in which samples in urine were simply diluted using water at a ratio of 1:5. Dilute-and-shoot is a simple method for screening of urine specimens, but it does not remove endogenous and possibly interfering compounds, 
which could contribute to the misidentification or nonidentification of some samples.

Use of a more thorough sample preparation may have resulted in improved results for the screening of the blind-spiked samples.

\subsection{Conclusion}

Validation of a method for screening and confirmation of NPS and related compounds was completed following guidelines established by the Toxicology Subcommittee of OSAC. Studies were conducted on NPS in urine to assess linearity, accuracy (bias), precision, LOD, LOQ, freeze-thaw stability, ion suppression or enhancement due to matrix effects, and carryover. While not all compounds were able to be fully validated using the method, a significant majority of the compounds in each mixture were successfully validated. It was expected that the positive-mode method would not be applicable to all NPS of interest given their wide variety of physiochemical properties, however, the results demonstrate that a mixture approach is a viable technique for validation of a screening and confirmation method for a significantly large number of NPS and related compounds.

The validated method was also used in conjunction with the compound database and HRMS spectral library that was developed (see Chapter 3) to qualitatively screen two series of 20 blind-spiked specimens in human urine. The qualitative screening was conducted as a preliminary test of applicability to real-world specimens. Positive identification was achieved for the majority of the compounds present in the specimens, indicating potential for real-world applicability. It is believed that identification could be further improved through curation of the MS/MS spectral data in the HRMS library to remove ions from compounds present in the background. However, as a preliminary test, 
applicability of the database and HRMS spectral library used with the validated method for screening and confirmation was confirmed.

Table 11. LOD and LOQ values for compounds in Mix 1.

\begin{tabular}{|c|c|c|}
\hline Compound Name & LOD (ng/mL) & LOQ (ng/mL) \\
\hline$\alpha$-Pyrrolidinopentiophenone metabolite 1 & 0.6 & 1.8 \\
\hline 25I-NBMD & 0.2 & 0.6 \\
\hline 3-Methylbuphedrone & 0.5 & 1.6 \\
\hline 4-APDB & 2.5 & 7.5 \\
\hline 4-fluoro- $\alpha$-Pyrrolidinobutiophenone & 0.4 & 1.2 \\
\hline 4'-fluoro- $\alpha$-Pyrrolidinopropiophenone & 0.5 & 1.4 \\
\hline 4-hydroxy MET & 0.8 & 2.4 \\
\hline 4-methoxy PV8 & 0.7 & 2.2 \\
\hline 4-methoxy- $\alpha$-Pyrrolidinopentiophenone & 0.7 & 2.1 \\
\hline 4-Methyl- $\alpha$-ethylaminobutiophenone & 0.2 & 0.6 \\
\hline 5-fluoro SDB-006 & 0.7 & 2.2 \\
\hline 5-Fluoropentylindole & 0.6 & 1.9 \\
\hline 5-MAPB & 0.7 & 2.0 \\
\hline $\mathrm{AB}-005$ & 0.3 & 0.9 \\
\hline AM-2233 azepane isomer & 0.5 & 1.5 \\
\hline AM694 N-(5-hydroxypentyl) metabolite & 0.6 & 1.8 \\
\hline AMT & 2.9 & 8.8 \\
\hline Benocyclidine & 1.4 & 4.2 \\
\hline Flubromazepam & 0.3 & 0.8 \\
\hline JWH 031 2'-isomer & 0.7 & 2.1 \\
\hline JWH $081 \mathrm{~N}$-(5-hydroxypentyl) metabolite & 1.0 & 3.0 \\
\hline JWH 200 5-hydroxyindole metabolite & 0.7 & 2.2 \\
\hline JWH 203 & 1.0 & 3.1 \\
\hline N-Methyltryptamine & 2.0 & 6.1 \\
\hline NPB-22 & 0.5 & 1.5 \\
\hline PB-22 6-hydroxyisoquinoline isomer & 0.5 & 1.5 \\
\hline PCMPA & 0.5 & 1.5 \\
\hline THCA-A & 1.4 & 4.1 \\
\hline UR-144 N-heptyl analog & 0.8 & 2.3 \\
\hline
\end{tabular}


Table 12. LOD and LOQ values for compounds in Mix 2.

\begin{tabular}{|c|c|c|}
\hline Compound Name & LOD (ng/mL) & LOQ (ng/mL) \\
\hline 2,3-methylenedoxy pyrovalerone & 0.7 & 2.1 \\
\hline 25I-NBF & 0.3 & 0.9 \\
\hline $2 \mathrm{C}-\mathrm{T}-2$ & 0.5 & 1.4 \\
\hline $2 \mathrm{C}-\mathrm{T}-4$ & 0.5 & 1.5 \\
\hline 2-fluoromethcathinone & 2.2 & 6.8 \\
\hline $\begin{array}{c}\text { 3,4-dimethoxy- } \alpha- \\
\text { pyrrolidinopentiophenone }\end{array}$ & 1.7 & 4.4 \\
\hline 3-methyl- $\alpha$-pyrrolidinopropiophenone & 0.8 & 2.4 \\
\hline 4'-methyl-N-methylhexanophenone & 0.7 & 2.1 \\
\hline 4-ethyl-N,N-dimethylcathinone & 1.2 & 3.6 \\
\hline 4-hydroxy MiPT & 0.2 & 0.7 \\
\hline 4-MMC & 1.0 & 3.1 \\
\hline A-796260 & 0.4 & 1.3 \\
\hline AB-005 azepine isomer & 0.4 & 1.1 \\
\hline $\begin{array}{l}\text { AB-FUBINACA 3-fluorobenzyl } \\
\text { isomer }\end{array}$ & 0.8 & 2.9 \\
\hline ADB-PINACA isomer 1 & 0.4 & 1.3 \\
\hline AKB48 N-(5-fluoropentyl) analog & 0.4 & 1.1 \\
\hline Clencyclohexerol & 0.2 & 0.7 \\
\hline EG-018 & 0.3 & 1.0 \\
\hline $\begin{array}{l}\text { JWH } 018 \text { N-(5-hydroxypentyl) } \\
\text { metabolite }\end{array}$ & 0.5 & 1.6 \\
\hline JWH 018 N-propanoic acid metabolite & 0.4 & 1.1 \\
\hline KM 233 & 0.3 & 1.0 \\
\hline Loperamide & 0.5 & 1.5 \\
\hline $\begin{array}{l}\text { MAM2201 N-pentanoic acid } \\
\text { metabolite }\end{array}$ & 0.3 & 0.9 \\
\hline N-Ethylbuphedrone & 0.8 & 2.4 \\
\hline $\mathrm{PCPr}$ & 0.4 & 1.1 \\
\hline RCS-4 2-methoxy isomer & 0.9 & 2.8 \\
\hline SER-601 & 0.1 & 0.3 \\
\hline UR-144 Degradant & 0.4 & 1.2 \\
\hline XLR11 N-(2-fluoropentyl) isomer & 0.4 & 1.3 \\
\hline$\Delta 8$-THC & 0.8 & 2.5 \\
\hline
\end{tabular}


Table 13. LOD and LOQ values for compounds in Mix 3.

\begin{tabular}{|c|c|c|}
\hline Compound Name & LOD (ng/mL) & LOQ (ng/mL) \\
\hline (R)-(-)-MT-45 & 0.5 & 1.4 \\
\hline 2,3-Dichlorophenylpiperazine & 0.6 & 1.7 \\
\hline 25H-NBOMe & 0.3 & 0.8 \\
\hline 2C-T & 1.3 & 3.9 \\
\hline 2-Methoxyamphetamine & 1.0 & 2.9 \\
\hline 3,4-Dimethylethcathinone & 0.3 & 0.8 \\
\hline 3C-P & 0.8 & 2.5 \\
\hline 4-Methoxyamphetamine & 0.8 & 2.3 \\
\hline 5-fluoro NNEI & 0.2 & 0.6 \\
\hline 9-octadecenamide/oleamide & 1.6 & 4.7 \\
\hline AB-CHMINACA & 0.8 & 2.3 \\
\hline AKB48 N-pentanoic acid metabolite & 0.3 & 0.8 \\
\hline Cathine & 1.2 & 3.8 \\
\hline Diclofensine & 0.2 & 0.7 \\
\hline FUB-PB-22 & 0.3 & 0.9 \\
\hline HMA & 1.3 & 3.9 \\
\hline MBZP & 0.6 & 1.9 \\
\hline Mephedrone & 0.4 & 1.2 \\
\hline N-methyl-2-AI & 0.7 & 2.0 \\
\hline NRG-3 & 0.4 & 1.1 \\
\hline Methylenedioxy Pyrovalerone metabolite 2 & 1.0 & 2.9 \\
\hline PB-22 N-(5-hydroxypentyl) metabolite & 0.3 & 1.0 \\
\hline RCS-4 N-(4-hydroxypentyl) metabolite & 1.0 & 3.0 \\
\hline UR-144 N-(2-chloropentyl) analog & 0.5 & 1.6 \\
\hline
\end{tabular}


Table 14. Stability data for Mix 2 compounds after three freeze-thaw cycles.

\begin{tabular}{|c|c|c|}
\hline & \multicolumn{2}{|c|}{ Bias (\%) } \\
\hline Compound Name & Low (5 ng/mL) & $\operatorname{High}(120 \mathrm{ng} / \mathrm{mL})$ \\
\hline 2,3-methylenedoxy pyrovalerone & 9.3 & 4.6 \\
\hline $25 \mathrm{I}-\mathrm{NBF}$ & 9.6 & 7.9 \\
\hline $2 \mathrm{C}-\mathrm{T}-2$ & 14.3 & 6.9 \\
\hline $2 \mathrm{C}-\mathrm{T}-4$ & 9.8 & 5.0 \\
\hline 2-fluoromethcathinone & 25.9 & 5.1 \\
\hline $\begin{array}{c}\text { 3,4-dimethoxy- } \alpha- \\
\text { pyrrolidinopentiophenone }\end{array}$ & 4.8 & 5.6 \\
\hline $\begin{array}{c}\text { 3-methyl- } \alpha- \\
\text { pyrrolidinopropiophenone }\end{array}$ & 11.5 & 6.5 \\
\hline $\begin{array}{c}\text { 4'-methyl-N- } \\
\text { methylhexanophenone }\end{array}$ & 13.8 & 7.2 \\
\hline 4-ethyl-N,N-dimethylcathinone & 14.3 & 9.6 \\
\hline 4-hydroxy MiPT & 16.8 & 16.3 \\
\hline 4-MMC & 4.9 & 4.0 \\
\hline A-796260 & 7.0 & 19.5 \\
\hline AB-005 azepine isomer & 20.7 & 7.6 \\
\hline $\begin{array}{l}\text { AB-FUBINACA 3-fluorobenzyl } \\
\text { isomer }\end{array}$ & 4.7 & 14.0 \\
\hline ADB-PINACA isomer 1 & 9.9 & 5.7 \\
\hline $\begin{array}{l}\text { AKB48 N-(5-fluoropentyl) } \\
\text { analog }\end{array}$ & 38.5 & 9.4 \\
\hline Clencyclohexerol & 19.4 & 17.7 \\
\hline EG-018 & 84.1 & 0.2 \\
\hline $\begin{array}{l}\text { JWH } 018 \mathrm{~N} \text {-(5-hydroxypentyl) } \\
\text { metabolite }\end{array}$ & 18.3 & 9.1 \\
\hline $\begin{array}{l}\text { JWH } 018 \text { N-propanoic acid } \\
\text { metabolite }\end{array}$ & 16.7 & 19.9 \\
\hline KM 233 & 73.6 & 12.8 \\
\hline Loperamide & 3.9 & 4.9 \\
\hline $\begin{array}{l}\text { MAM2201 N-pentanoic acid } \\
\text { metabolite }\end{array}$ & 12.1 & 4.8 \\
\hline N-Ethylbuphedrone & 17.9 & 11.2 \\
\hline $\mathrm{PCPr}$ & 8.5 & 10.9 \\
\hline RCS-4 2-methoxy isomer & 18.4 & 5.1 \\
\hline SER-601 & 82.7 & 3.2 \\
\hline UR-144 Degradant & 52.3 & 7.5 \\
\hline $\begin{array}{l}\text { XLR11 N-(2-fluoropentyl) } \\
\text { isomer }\end{array}$ & 47.5 & 3.6 \\
\hline$\triangle 8$-THC & 67.0 & 19.1 \\
\hline
\end{tabular}


Table 15. Stability data for Mix 3 compounds after three freeze-thaw cycles.

\begin{tabular}{|c|c|c|}
\hline & \multicolumn{2}{|c|}{ Bias (\%) } \\
\hline Compound Name & Low (5 ng/mL) & High $(120 \mathrm{ng} / \mathrm{mL})$ \\
\hline (R)-(-)-MT-45 & 4.1 & 5.5 \\
\hline 2,3-Dichlorophenylpiperazine & 7.1 & 8.0 \\
\hline $25 \mathrm{H}-\mathrm{NBOMe}$ & 2.5 & 8.0 \\
\hline $2 \mathrm{C}-\mathrm{T}$ & 3.1 & 4.6 \\
\hline 2-Methoxyamphetamine & 8.5 & 3.9 \\
\hline 3,4-Dimethylethcathinone & 10.6 & 10.9 \\
\hline 3C-P & 1.6 & 5.4 \\
\hline 4-Methoxyamphetamine & 5.3 & 18.8 \\
\hline 5-fluoro NNEI & 5.9 & 12.9 \\
\hline 9-octadecenamide/oleamide & 32.8 & 11.8 \\
\hline AB-CHMINACA & 17.7 & 10.0 \\
\hline AKB48 N-pentanoic acid metabolite & 29.2 & 14.6 \\
\hline Cathine & 16.1 & 14.5 \\
\hline Diclofensine & 5.6 & 3.3 \\
\hline FUB-PB-22 & 9.7 & 12.5 \\
\hline HMA & 8.3 & 6.3 \\
\hline MBZP & 1.9 & 9.1 \\
\hline Mephedrone & 7.9 & 11.7 \\
\hline $\begin{array}{l}\text { Methylenedioxy Pyrovalerone } \\
\text { metabolite } 2\end{array}$ & 6.1 & 15.7 \\
\hline N-methyl-2-AI & 12.5 & 13.8 \\
\hline NRG-3 & 5.5 & 15.1 \\
\hline PB-22 N-(5-hydroxypentyl) metabolite & 10.5 & 9.5 \\
\hline RCS-4 N-(4-hydroxypentyl) metabolite & 10.6 & 13.3 \\
\hline UR-144 N-(2-chloropentyl) analog & 18.3 & 5.5 \\
\hline
\end{tabular}


Table 16. Ion suppression and enhancement (matrix effects) values for compounds in Mix 1.

\begin{tabular}{|c|c|c|}
\hline & \multicolumn{2}{|c|}{ M.E. (\%) } \\
\hline Compound Name & Low $(5 \mathrm{ng} / \mathrm{mL})$ & High $(100 \mathrm{ng} / \mathrm{mL})$ \\
\hline 25I-NBMD & 8.04 & -27.14 \\
\hline 3-Methylbuphedrone & -18.57 & -21.87 \\
\hline 4-APDB & -52.74 & -47.14 \\
\hline 4-fluoro- $\alpha$-Pyrrolidinobutiophenone & -29.12 & -32.58 \\
\hline 4'-fluoro- $\alpha$-Pyrrolidinopropiophenone & -22.64 & -28.71 \\
\hline 4-hydroxy MET & -38.35 & -45.23 \\
\hline 4-methoxy PV8 & 4.16 & -30.44 \\
\hline 4-methoxy- $\alpha$-Pyrrolidinopentiophenone & -7.17 & -22.65 \\
\hline 4-Methyl- $\alpha$-ethylaminobutiophenone & -22.95 & -39.56 \\
\hline 5-fluoro SDB-006 & -20.65 & -13.49 \\
\hline 5-Fluoropentylindole & -7.85 & -6.14 \\
\hline 5-MAPB & -11.10 & -26.86 \\
\hline AB-005 & 113.44 & -22.24 \\
\hline AM-2233 azepane isomer & -10.89 & -40.69 \\
\hline AM694 N-(5-hydroxypentyl) metabolite & -5.16 & -2.61 \\
\hline AMT & -30.27 & -34.55 \\
\hline Benocyclidine & 21.52 & -25.53 \\
\hline Flubromazepam & -55.79 & -27.25 \\
\hline JWH 031 2'-isomer & -19.97 & 13.60 \\
\hline JWH $081 \mathrm{~N}$-(5-hydroxypentyl) metabolite & 1.70 & 11.84 \\
\hline JWH 200 5-hydroxyindole metabolite & -24.72 & -12.64 \\
\hline JWH 203 & 1.14 & -3.39 \\
\hline N-Methyltryptamine & -92.05 & -54.53 \\
\hline NPB-22 & 49.11 & -3.00 \\
\hline PB-22 6-hydroxyisoquinoline isomer & 53.07 & -2.49 \\
\hline PCMPA & -3.68 & -22.44 \\
\hline THCA-A & -28.26 & -3.48 \\
\hline UR-144 N-heptyl analog & -46.81 & -2.99 \\
\hline$\alpha$-Pyrrolidinopentiophenone metabolite 1 & -20.50 & -28.36 \\
\hline
\end{tabular}


Table 17. Ion suppression and enhancement (matrix effects) values for compounds in Mix 2.

\begin{tabular}{|c|c|c|}
\hline & \multicolumn{2}{|c|}{ M.E. (\%) } \\
\hline Compound Name & Low $(5 \mathrm{ng} / \mathrm{mL})$ & High $(100 \mathrm{ng} / \mathrm{mL})$ \\
\hline 2,3-methylenedoxy pyrovalerone & -15.0 & -40.3 \\
\hline $25 \mathrm{I}-\mathrm{NBF}$ & -52.5 & -60.7 \\
\hline $2 \mathrm{C}-\mathrm{T}-2$ & -62.7 & -63.0 \\
\hline $2 \mathrm{C}-\mathrm{T}-4$ & -76.0 & -73.1 \\
\hline 2-fluoromethcathinone & -16.9 & -41.4 \\
\hline 3,4-dimethoxy- $\alpha$-pyrrolidinopentiophenone & 19.2 & -43.2 \\
\hline 3-methyl- $\alpha$-pyrrolidinopropiophenone & 3.1 & -48.0 \\
\hline 4'-methyl-N-methylhexanophenone & -75.1 & -79.3 \\
\hline 4-ethyl-N,N-dimethylcathinone & -41.2 & -66.0 \\
\hline 4-hydroxy MiPT & -8.2 & -15.8 \\
\hline 4-MMC & -68.4 & -74.1 \\
\hline A-796260 & -7.4 & -41.3 \\
\hline AB-005 azepine isomer & -54.7 & -59.9 \\
\hline AB-FUBINACA 3-fluorobenzyl isomer & -19.2 & -38.3 \\
\hline ADB-PINACA isomer 1 & 9.1 & -16.7 \\
\hline AKB48 N-(5-fluoropentyl) analog & -8.3 & -5.1 \\
\hline Clencyclohexerol & -71.3 & -52.1 \\
\hline EG-018 & -16.9 & -8.0 \\
\hline JWH 018 N-(5-hydroxypentyl) metabolite & -1.5 & -37.2 \\
\hline JWH 018 N-propanoic acid metabolite & -19.0 & -32.2 \\
\hline KM 233 & -18.8 & -18.6 \\
\hline Loperamide & -2.1 & -34.6 \\
\hline MAM2201 N-pentanoic acid metabolite & -3.4 & -18.2 \\
\hline N-Ethylbuphedrone & -43.6 & -30.2 \\
\hline $\mathrm{PCPr}$ & -32.9 & -65.6 \\
\hline RCS-4 2-methoxy isomer & 16.3 & -8.4 \\
\hline SER-601 & -3.1 & -5.2 \\
\hline UR-144 Degradant & 19.4 & -5.0 \\
\hline XLR11 N-(2-fluoropentyl) isomer & 18.0 & -5.5 \\
\hline$\Delta 8-\mathrm{THC}$ & -16.6 & -23.4 \\
\hline
\end{tabular}


Table 18. Ion suppression and enhancement (matrix effects) values for compounds in Mix 3.

\begin{tabular}{|c|c|c|}
\hline & \multicolumn{2}{|c|}{ M.E. (\%) } \\
\hline Compound Name & Low $(5 \mathrm{ng} / \mathrm{mL})$ & High $(100 \mathrm{ng} / \mathrm{mL})$ \\
\hline (R)-(-)-MT-45 & 32.5 & -30.5 \\
\hline 2,3-Dichlorophenylpiperazine & -74.3 & -76.4 \\
\hline $25 \mathrm{H}-\mathrm{NBOMe}$ & 21.6 & -29.7 \\
\hline $2 \mathrm{C}-\mathrm{T}$ & -3.7 & -45.6 \\
\hline 2-Methoxyamphetamine & -45.9 & -52.8 \\
\hline 3,4-Dimethylethcathinone & -38.5 & -48.5 \\
\hline $3 \mathrm{C}-\mathrm{P}$ & 64.3 & -26.8 \\
\hline 4-Methoxyamphetamine & -67.6 & -58.8 \\
\hline 5-fluoro NNEI & -30.6 & -18.0 \\
\hline 9-octadecenamide/oleamide & -3.1 & -6.4 \\
\hline AB-CHMINACA & -3.0 & -4.9 \\
\hline $\begin{array}{l}\text { AKB48 N-pentanoic acid } \\
\text { metabolite }\end{array}$ & -4.2 & -5.5 \\
\hline Cathine & -70.5 & -19.9 \\
\hline Diclofensine & -24.4 & -52.6 \\
\hline FUB-PB-22 & -20.6 & -11.2 \\
\hline HMA & -36.6 & 8.7 \\
\hline MBZP & -6.8 & -32.2 \\
\hline Mephedrone & -73.3 & -80.1 \\
\hline $\begin{array}{l}\text { Methylenedioxy Pyrovalerone } \\
\text { metabolite } 2\end{array}$ & 9.9 & -49.4 \\
\hline N-methyl-2-AI & -64.4 & -67.0 \\
\hline NRG-3 & -44.2 & -57.0 \\
\hline $\begin{array}{l}\text { PB-22 N-(5-hydroxypentyl) } \\
\text { metabolite }\end{array}$ & 2.3 & -32.7 \\
\hline $\begin{array}{l}\text { RCS-4 N-(4-hydroxypentyl) } \\
\text { metabolite }\end{array}$ & -20.1 & -16.1 \\
\hline $\begin{array}{l}\text { UR-144 N-(2-chloropentyl) } \\
\text { analog }\end{array}$ & -40.7 & -3.6 \\
\hline
\end{tabular}


Table 19. Results of blind-spiked urine specimen screening for Set 1 . Specimens were prepared at concentrations of 2 or $20 \mathrm{ng} / \mathrm{mL}$.

\begin{tabular}{|c|c|c|}
\hline Sample & True ID & Correct \\
\hline 1 & Mephedrone & No \\
\hline 2 & $\begin{array}{l}\text { PB-22 N-(5-hydroxypentyl) } \\
\text { metabolite }\end{array}$ & Yes \\
\hline 3 & MDPV metabolite 2 & Yes \\
\hline 4 & BB-22 & Yes \\
\hline 5 & AKB48 N-pentanoic acid & No \\
\hline 6 & Blank & Yes \\
\hline 7 & $\begin{array}{l}\text { PB-22 4-hydroxyisoquinoline } \\
\text { isomer }\end{array}$ & Yes \\
\hline 8 & $\begin{array}{l}\text { PB-22 5-hydroxyisoquinoline } \\
\text { isomer }\end{array}$ & Yes \\
\hline 9 & AB-FUBINACA & Yes \\
\hline 10 & MDPV & Yes \\
\hline 11 & 25H-NBOMe & No \\
\hline 12 & MDPV & No \\
\hline 13 & MDPV metabolite 1 & Yes \\
\hline 14 & $2 \mathrm{C}-\mathrm{T}$ & No \\
\hline 15 & PCEEA & Yes \\
\hline 16 & Blank & Yes \\
\hline 17 & $\begin{array}{l}\text { 5-fluoro PB-22 3-carboxyindole } \\
\text { metabolite }\end{array}$ & No \\
\hline 18 & 25H-NBOMe & Yes \\
\hline 19 & $\begin{array}{l}\text { PB-22 N-(5-hydroxypentyl) } \\
\text { metabolite }\end{array}$ & Yes \\
\hline 20 & $2 \mathrm{C}-\mathrm{T}$ & No \\
\hline
\end{tabular}


Table 20. Results of blind-spiked urine specimen screening for Set 2. Specimens were prepared at concentrations of $200 \mathrm{ng} / \mathrm{mL}$.

\begin{tabular}{|c|c|c|}
\hline Sample & True ID & Correct \\
\hline 1 & Mephedrone & No \\
\hline 2 & PB-22 N-(5-hydroxypentyl) metabolite & Yes \\
\hline 3 & MDPV metabolite 2 & Yes \\
\hline 4 & BB-22 & Yes \\
\hline 5 & BKB48 N-pentanoic acid & No \\
\hline 6 & PB-22 4-hydroxyisoquinoline isomer & Yes \\
\hline 7 & PB-22 5-hydroxyisoquinoline isomer & Yes \\
\hline 8 & AB-FUBINACA & Yes \\
\hline 9 & 25H-NBOMe & Yes \\
\hline 10 & MDPV & No \\
\hline 11 & MDPV metabolite 1 & Yes \\
\hline 12 & 2C-T & Yes \\
\hline 13 & PCEEA & No \\
\hline 14 & Blank & Yes \\
\hline 15 & 25H-NBOMe & Yes \\
\hline 16 & 2C-T & Yes \\
\hline 17 & 5-fluoro PB-22 3-carboxyindole metabolite & No \\
\hline 18 & PB-22 N-(5-hydroxypentyl) metabolite & Yes \\
\hline 19 & & Yes \\
\hline 20 & &
\end{tabular}




\section{DEVELOPMENT OF 2D-LC METHOD}

\subsection{Introduction}

Novel psychoactive substances (NPS), also known as "designer drugs" and "legal highs," refer to emerging drugs of abuse that are variations of existing compounds intended to cause a CNS psychotropic effect. Major categories of NPS include synthetic cannabinoids, cathinones, piperazines, tryptamines, and phenethylamines. ${ }^{40,69}$ As more and more NPS are synthesized and appear on the market, analytical complications arise, due to the need to separate and identify compounds with minor or novel structural differences. These changes may include the alteration of a single functional group or the shifting of a functional group on the molecule to create isomers. Such alterations may render some NPS so comparable in structure and physicochemical properties that they cannot be separated using traditional techniques such as gas chromatography (GC) or liquid chromatography (LC) and therefore will not be detected as individual

compounds. ${ }^{70-72}$ NPS of interest that are in complex matrices may also provide an analytical challenge, as a multitude of components can interfere with the detection of the analyte of interest. ${ }^{98}$

Synthetic cannabinoids (SC) were originally developed for research purposes to study the pharmacology of compounds that interacted with CB1 and/or CB2 receptors. ${ }^{25}$ $\mathrm{SC}$ in particular pose a significant challenge to chromatographic separations due to the large number of compounds and metabolites that exist, and even more so due to the presence of numerous isomers for many SC. ${ }^{155-157}$ For example, the naphthoylindole derivatives have increased in street popularity and include compounds such as JWH 018 , JWH 019, JWH 080, and JWH 250, all of which share a structure with variation only on 
the indole alkyl side chain. ${ }^{77,158}$ Isomers of SC may also have similar chromatographic retention times, making baseline separation difficult. Such co-elution can be a major analytical challenge during traditional one-dimensional (1D) chromatographic analyses of large numbers of NPS, including SC. This can be problematic for identification purposes if the coeluting compounds are unknown or previously unreported, particularly if they are indistinguishable using accurate mass data (e.g., in the case of isomeric derivatives). ${ }^{25,143}$

Currently, analytical methods for detecting large numbers of SC in a single analysis are not as common as methods intended for a small group of select SC. ${ }^{26,156,157,159,160}$ One such approach was applied to a mixture containing 54 SC and 21 NPS from other classes. ${ }^{26}$ However, this screening method relied on the use of known retention times and accurate masses of precursor and qualifier ions. Such is generally the case for other methods requiring the use of libraries and databases which depend on known accurate masses and retention times. ${ }^{26,143,157,161}$ This reliance on library data becomes problematic when screening for unknown or previously unreported SC, since there exist many isomers and related compounds with similar accurate masses, fragmentation patterns, and/or chromatographic retention times. In these instances, it is important to have an improved initial separation, so that compounds may be analyzed individually once they reach the detector. ${ }^{45,77}$

Two-dimensional liquid chromatography (2D-LC) has been proposed as a method to improve separation and resolution of complex mixtures prior to further mass spectral analysis. $^{73,74}$ This method has been shown to be effective in the separation of pharmaceuticals and small molecules such as methamphetamine, anti-retroviral drugs, traditional Chinese medicinal preparations, and antiretroviral drugs from complex 
samples. ${ }^{71,72,75,88-90,92,105}$ In the case of NPS such as SC, the improved resolving power that 2D-LC can provide may be useful for chromatographically resolving these compounds prior to characterization by mass spectrometry.

To date, there are no reports on the use of 2D-LC specifically for separation of coeluting or isomeric NPS, although the demonstrated utility of 2D-LC for other classes of xenobiotics indicates that such a method could be effective. ${ }^{81,89,94,162}$ In the present study, the separation of both isomeric and non-isobaric mixtures of SC was examined using a 2D-LC separation system coupled with high resolution mass spectrometry (HRMS). To perform the separation, two orthogonal LC column systems, or dimensions, were joined to improve the resolving power of the overall separation by combining the power of each dimension. ${ }^{79,80,86}$ This work does not present a validated method, but rather serves as a proof-of-concept investigation. Results of this study confirm the potential utility of comprehensive 2D-LC combined with HRMS for the separation and identification of co-eluting, non-isobaric and isomeric SC.

\subsection{Materials and Methods}

\subsubsection{Chemicals}

Methanol, acetonitrile, and HPLC water (all Optima LC/MS grade) were purchased from Fisher Scientific (Fair Lawn, NJ, USA). Trifluoroacetic acid (TFA) was purchased from Sigma-Aldrich (St. Louis, MO, USA).

\subsubsection{Standards}

The synthetic cannabinoids JWH 073 2'-naphthyl-N-(1,1-dimethylethyl) isomer, JWH 080, JWH 203, FUB-144, PB-22, MAM 2201 N-(2-fluoropentyl) isomer, and XLR-12, in addition to five isomers of the synthetic cannabinoid JWH 019 (i.e., N-(2-fluorohexyl), 
$\mathrm{N}$-(3-fluorohexyl), $\mathrm{N}$-(4-fluorohexyl), N-(5-fluorohexyl), and N-(6-fluorohexyl) isomers) were obtained as neat solids from Cayman Chemical (Ann Arbor, MI, USA). Individual stock solutions of the standards were prepared at concentrations of $1 \mu \mathrm{g} / \mathrm{mL}$ in methanol.

Stock solutions of the SC were used to create individual component working solutions as well as three test mixes, each containing five individual components. In previous $1 \mathrm{D}-\mathrm{LC}$ work, very close retention times were observed for the individual components of each mix using a Zorbax Eclipse Plus RRHD C 18 column ( 3.0 x 100 mm; $1.8 \mu \mathrm{m}$; Agilent Technologies, Santa Clara, CA). Retention times were collected over 20 min using a gradient that reached $90 \% \mathrm{~B}$ at $9.5 \mathrm{~min}$, then held for the final $6.5 \mathrm{~min}$. These retention times are given in Table 21. In other words, the separation of all the constituents in the mixtures described here was not achievable by conventional means. Co-elution (CE) Mix 1 contained five JWH 019 isomers, while CE Mixes 2 and 3 each contained five non-isobaric (but co-eluting) synthetic cannabinoids. The composition of each mix is shown in Table 21 and the structures of all compounds examined are shown in Figure 8. The individual working solutions and mixes were prepared in $\mathrm{MeOH}: \mathrm{H}_{2} \mathrm{O}$ $(50: 50 \mathrm{v} / \mathrm{v})$ with each component present at a concentration of $1 \mu \mathrm{g} / \mathrm{mL}$. 
Table 21. Synthetic cannabinoids present in each co-elution (CE) mix.

\begin{tabular}{|c|c|c|c|c|c|}
\hline & Compound Name & ${ }^{a}$ Peak \# & $\begin{array}{l}\text { Molecular } \\
\text { Formula }\end{array}$ & $\begin{array}{l}\text { Exact Mass } \\
\text { (Da) }\end{array}$ & ${ }^{b} \mathbf{R T}$ (min) \\
\hline \multirow{5}{*}{$\begin{array}{c}\text { CE } \\
\text { Mix } \\
1\end{array}$} & $\begin{array}{l}\text { JWH } 019 \text { N-(2- } \\
\text { fluorohexyl) isomer }\end{array}$ & 1 & $\mathrm{C}_{25} \mathrm{H}_{24} \mathrm{FNO}$ & 373.1842 & 12.16 \\
\hline & $\begin{array}{l}\text { JWH } 019 \text { N-(3- } \\
\text { fluorohexyl) isomer }\end{array}$ & 2 & $\mathrm{C}_{25} \mathrm{H}_{24} \mathrm{FNO}$ & 373.1842 & 12.25 \\
\hline & $\begin{array}{l}\text { JWH } 019 \text { N-(4- } \\
\text { fluorohexyl) isomer }\end{array}$ & 3 & $\mathrm{C}_{25} \mathrm{H}_{24} \mathrm{FNO}$ & 373.1842 & 11.90 \\
\hline & $\begin{array}{l}\text { JWH } 019 \text { N-(5- } \\
\text { fluorohexyl) isomer }\end{array}$ & 4 & $\mathrm{C}_{25} \mathrm{H}_{24} \mathrm{FNO}$ & 373.1842 & 11.75 \\
\hline & $\begin{array}{c}\text { JWH } 019 \text { N-(6- } \\
\text { fluorohexyl) isomer }\end{array}$ & 5 & $\mathrm{C}_{25} \mathrm{H}_{24} \mathrm{FNO}$ & 373.1842 & 11.76 \\
\hline \multirow{5}{*}{$\begin{array}{c}\mathrm{CE} \\
\mathrm{Mix} \\
2\end{array}$} & JWH 080 & 6 & $\mathrm{C}_{24} \mathrm{H}_{23} \mathrm{NO}_{2}$ & 357.1729 & 12.16 \\
\hline & JWH 203 & 7 & $\mathrm{C}_{21} \mathrm{H}_{22} \mathrm{CINO}$ & 339.1390 & 12.22 \\
\hline & PB-22 & 8 & $\mathrm{C}_{23} \mathrm{H}_{22} \mathrm{~N}_{2} \mathrm{O}_{2}$ & 346.1681 & 11.88 \\
\hline & $\begin{array}{l}\text { MAM 2201 N-(2- } \\
\text { fluoropentyl) isomer }\end{array}$ & 9 & $\mathrm{C}_{25} \mathrm{H}_{24} \mathrm{FNO}$ & 373.1842 & 12.20 \\
\hline & XLR12 & 10 & $\mathrm{C}_{20} \mathrm{H}_{24} \mathrm{~F}_{3} \mathrm{NO}$ & 351.1810 & 12.01 \\
\hline \multirow{5}{*}{$\begin{array}{c}\text { CE } \\
\text { Mix } \\
\mathbf{3}\end{array}$} & $\begin{array}{l}\text { JWH } 073 \text { 2'-naphthyl-N- } \\
\text { (1,1-dimethylethyl) isomer }\end{array}$ & 11 & $\mathrm{C}_{23} \mathrm{H}_{21} \mathrm{NO}$ & 327.1623 & 12.20 \\
\hline & $\begin{array}{c}\text { JWH } 019 \text { N-(2- } \\
\text { fluorohexyl) isomer }\end{array}$ & 1 & $\mathrm{C}_{25} \mathrm{H}_{24} \mathrm{FNO}$ & 373.1842 & 12.16 \\
\hline & JWH 080 & 6 & $\mathrm{C}_{24} \mathrm{H}_{23} \mathrm{NO}_{2}$ & 357.1729 & 12.16 \\
\hline & JWH 203 & 7 & $\mathrm{C}_{21} \mathrm{H}_{22} \mathrm{ClNO}$ & 339.1390 & 12.22 \\
\hline & FUB-144 & 12 & $\mathrm{C}_{23} \mathrm{H}_{24} \mathrm{FNO}$ & 349.1842 & 12.26 \\
\hline
\end{tabular}

aPeak number as indicated in Figures 13, 14, and 15.

${ }^{b}$ Retention time observed in 1D-LC separation performed in Chapter 4. 


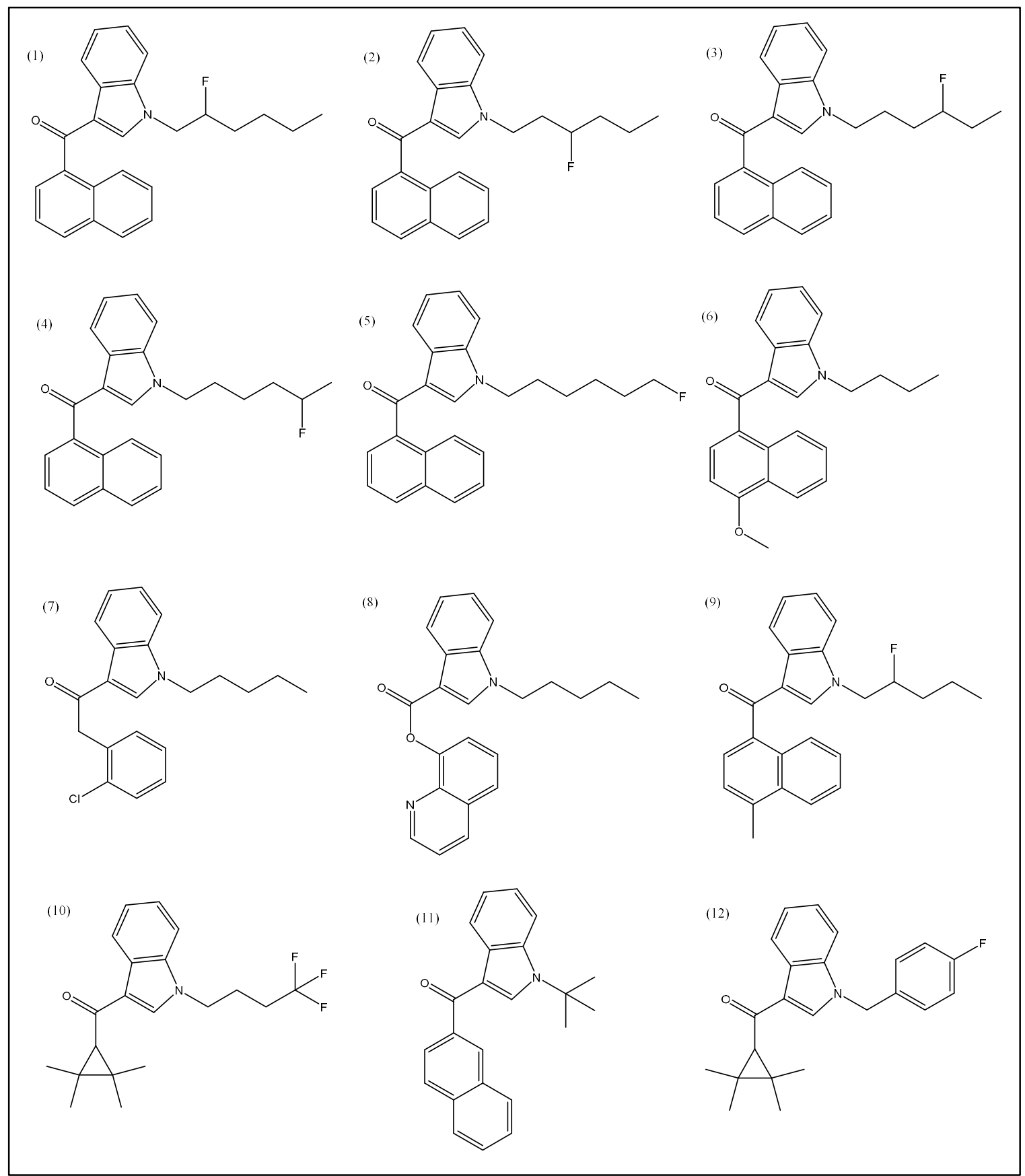

Figure 8. Structures of NPS in CE mixes (see Table 21 for mixture compositions): (1) JWH 019 N-(2fluorohexyl) isomer, (2) JWH $019 \mathrm{~N}$-(3-fluorohexyl) isomer, (3) JWH $019 \mathrm{~N}$-(4-fluorohexyl) isomer, (4) JWH 019 N-(5-fluorohexyl) isomer, (5) JWH 019 N-(6-fluorohexyl) isomer, (6) JWH 080, (7) JWH 203, (8) PB-22, (9) MAM2201 N-(2-fluoropentyl) isomer, (10) XLR12, (11) JWH 073 2'-naphthyl-N-(1,1dimethylethylisomer), and (12) FUB-144. 


\subsubsection{Instrumentation}

Comprehensive, on-line 2D-LC analysis was performed with an Agilent Infinity 1290 2D-LC solution system (Agilent Technologies, Santa Clara, CA, USA) composed of two G4220A binary pumps, a G4226A temperature controlled autosampler, a G1316C thermostatted column compartment, and a G1170A Infinity Valve Drive with a twoposition eight-port switching valve (pressure limit 1200 bar; p/n 5067-4214) and two 20$\mu \mathrm{L}$ sampling loops. The configuration of the 2D-LC instruments is shown in Figure 9. Schematic of the two-position, four-port duo valve (right) used in conjunction with ta twoposition, six-port valve (left) to connect the two dimensions..

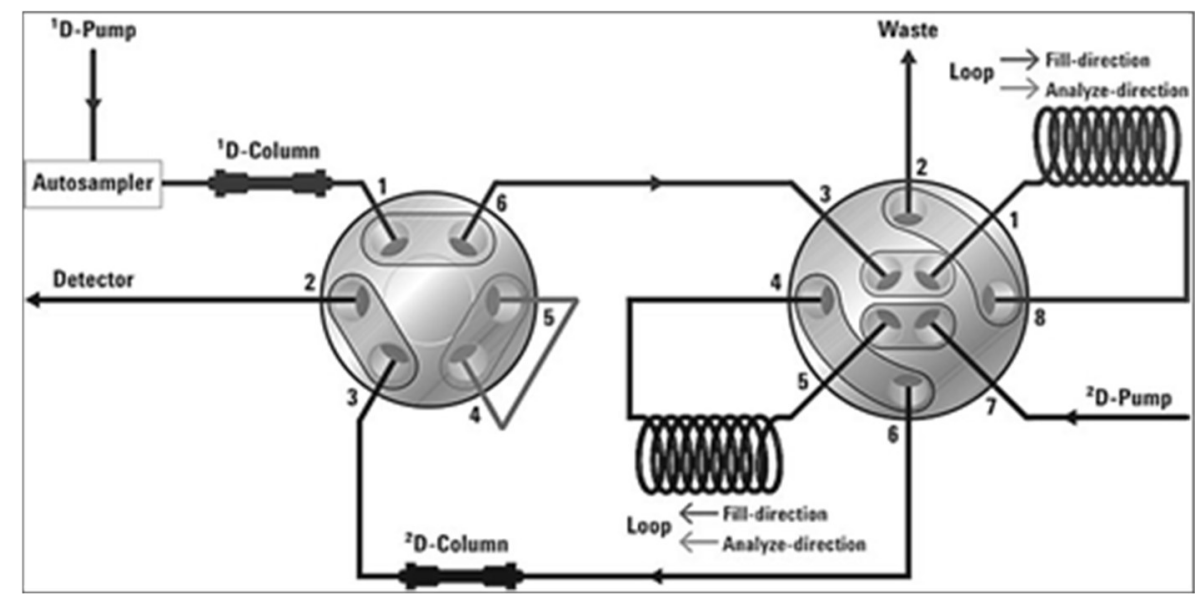

Figure 9. Schematic of the two-position, four-port duo valve (right) used in conjunction with ta twoposition, six-port valve (left) to connect the two dimensions.

For initial investigation of the columns chosen for each dimension, two Agilent 1290 diode array detectors were used, one after the ${ }^{1} \mathrm{D}$ separation and another following the ${ }^{2} \mathrm{D}$ separation. Once the initial investigations were completed, the $2 \mathrm{D}-\mathrm{LC}$ system utilized the same Agilent 1290 diode array detector placed directly after the first dimension. In addition, an Agilent 6530 Accurate-Mass Quadrupole Time-of-Flight 
(QTOF) mass spectrometer with Jetstream ESI source was placed directly after the second dimension and used as the ${ }^{2} \mathrm{D}$ detector (Figure 10). Positive mode ESI source settings were as follows: gas temperature, $300^{\circ} \mathrm{C}$; drying gas flow rate, $8 \mathrm{~L} / \mathrm{min}$; nebulizer pressure, $35 \mathrm{psi}$; sheath gas temperature, $350{ }^{\circ} \mathrm{C}$; sheath gas flow rate, 11 $\mathrm{L} / \mathrm{min}$; capillary voltage, $3500 \mathrm{~V}$; and nozzle voltage, $1000 \mathrm{~V}$. The QTOF fragmentor and skimmer voltages were 175 and $65 \mathrm{~V}$, respectively. Mass spectral data were collected in Full Scan mode with a mass range of $100-1700 \mathrm{~m} / \mathrm{z}$, and an acquisition rate of $1 \mathrm{spectrum/s}$. No collision energy was employed during the MS data collection.

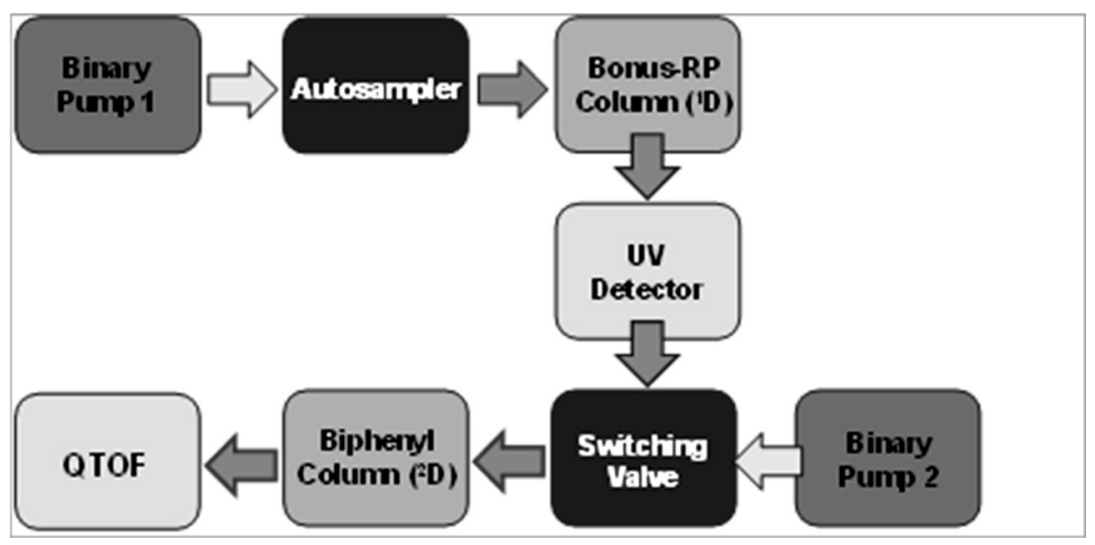

Figure 10. Schematic for the 2D-LC setup used in final system.

The software used to acquire, view, and analyze the analytical data included MassHunter Data Acquisition (version B.06.01), MassHunter Qualitative Analysis (version B.07.00), Open Lab Control Panel, and ChemStation (version C.01.07), all from Agilent Technologies (Santa Clara, CA, USA). Compounds were identified based on accurate mass with a mass tolerance of \pm 5 ppm using the Qualitative Analysis software. 


\subsubsection{Analytical Columns and Separation}

Separation of the components of each mix was initially examined in 1D-LC mode on each of the three columns listed below. These separations involved a 20-min gradient with (A) water $+0.1 \%$ formic acid and (B) $\mathrm{MeOH}+0.1 \%$ formic acid at a flow rate of $0.3 \mathrm{~mL} / \mathrm{min}$. The gradient ran $5 \%$ to $95 \% \mathrm{~B}$ from 0.5 to $9.5 \mathrm{~min}$, where it was then held at $95 \%$ for the remainder of the analysis.

Both the ${ }^{1} \mathrm{D}$ and ${ }^{2} \mathrm{D}$ separations employed reversed-phase LC conditions. Three columns were tested; a Zorbax Eclipse Plus RRHD column $(3.0$ x $100 \mathrm{~mm} ; 1.8 \mu \mathrm{m}$; Agilent Technologies, Santa Clara, CA), a Poroshell 120 Bonus-RP column (2.1 x 150mm, $2.7 \mu \mathrm{m}$; Agilent Technologies, Santa Clara, CA), and an Ascentis Express biphenyl column (2.1 mm x $100 \mathrm{~mm}, 2.7 \mu \mathrm{m}$; Supelco, Bellefonte, PA). In the final 2D method, the Poroshell 120 Bonus-RP column was chosen for ${ }^{1} \mathrm{D}$ and the Ascentis Express biphenyl column was chosen for ${ }^{2} \mathrm{D}$. Parameters for each dimension were optimized separately in 1D-LC separations before optimizing the complete 2D-LC separation system. Both dimensions used HPLC water with $0.1 \%$ TFA for the aqueous (A) solvent. For the ${ }^{1} \mathrm{D}$ separation, the (B) solvent was a mixture of acetonitrile (ACN) and water $(95: 5 v / v)$, and in the second dimension it was a mixture of methanol $(\mathrm{MeOH})$ and water $(95: 5 v / v)$. Columns were maintained at $40{ }^{\circ} \mathrm{C}$ in both dimensions. The temperature controlled autosampler was maintained at a temperature of $20^{\circ} \mathrm{C}$. In the first dimension, the flow rate was $0.1 \mathrm{~mL} / \mathrm{min}$, and the following solvent gradient was used for elution from the column: 5-5-80-80-95-95-100-10-5-5\% B from 0-0.5-19-20-30-37-42-42.01-45 min, respectively. The semi-shifted gradient used in the second dimension is shown in Figure 11 and the ${ }^{2} \mathrm{D}$ flow rate was $0.55 \mathrm{~mL} / \mathrm{min}$. The ${ }^{2} \mathrm{D}$ gradient stop time was 0.95 
min and the modulation time was $1.15 \mathrm{~min}$.

For each injection, UV spectra were collected with the diode array detector after the ${ }^{1} \mathrm{D}$ column at $210,220,230,240$, and $250 \mathrm{~nm}$. This was done to monitor any potential component separation that occurred solely in the first dimension. Working solutions of each compound were first run individually through the 2D-LC system to collect UV spectra and chromatograms for each compound. CE mixes were run under the same conditions and their data compared to those collected for the individual compounds. The volume of sample injected into the ${ }^{1} \mathrm{D}$ column was $2 \mu \mathrm{L}$.

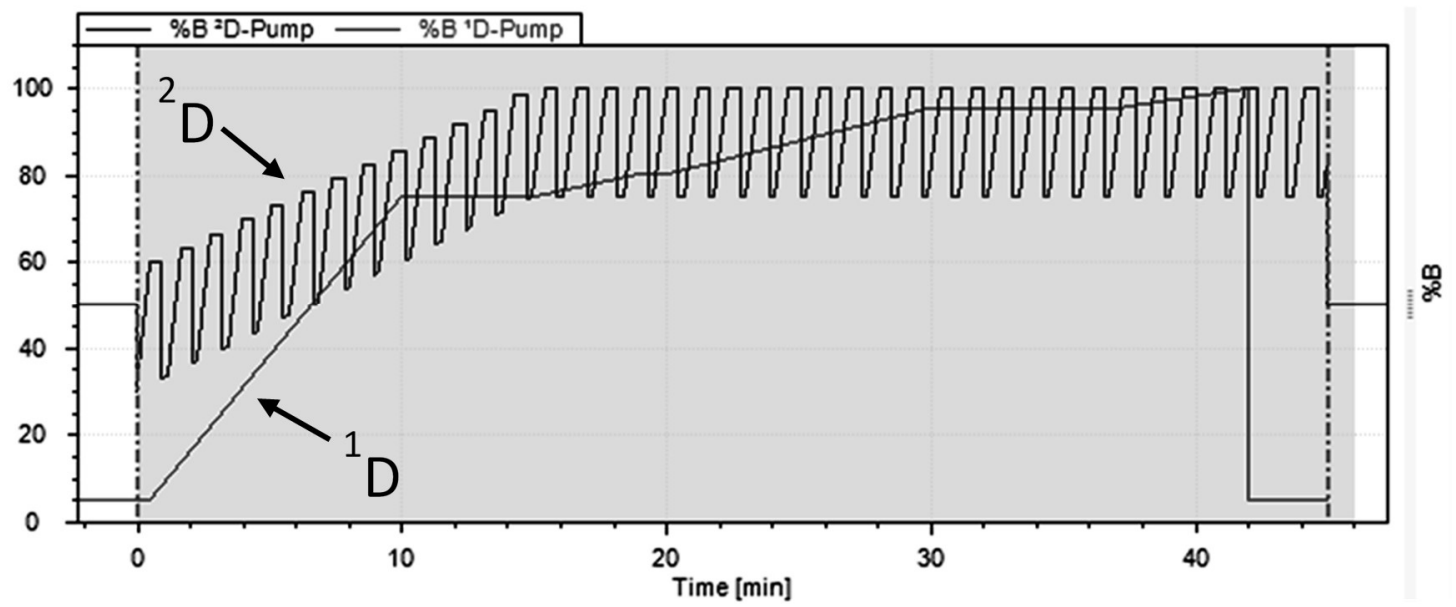

Figure 11. Solvent gradients used in the first and second dimensions of the 2D-LC separation.

\subsection{Results and Discussion}

Tentative determination of unknown compounds in samples can be conducted using a method known as suspect screening through the use of predicted or characteristic properties of compounds (i.e., accurate mass, isotope pattern, product ion spectrum). ${ }^{63}$ This method, though, is most effective when sample data are compared with previously collected data in a library or database. In the case of truly unknown and unreported 
compounds, it could be difficult to determine if the sample data represents a single analyte or a possible mixture of analytes or endogenous compounds, thus highlighting the need for adequate separation. ${ }^{46}$

The use of 2D-LC has several benefits for analytical separation of both small and large molecules. These include increased peak capacities, greater resolving power, separation of isomers and isobars, and better separation of compounds and metabolites, particularly in complex mixtures..$^{70,72,76,86,88,118}$ The increased separation power of 2D-LC derives from the combined resolution and peak capacities of the two orthogonal dimensions. There are two general approaches to 2D-LC; comprehensive and heartcutting separation. The present research focused on the use of comprehensive 2D-LC, which involves the complete transfer of all effluent from ${ }^{1} \mathrm{D}$ to ${ }^{2} \mathrm{D}$. This approach enables full automation of the chromatographic analysis, is preferred for use in non-targeted analyses, and minimizes analyte loss. Low analyte loss makes comprehensive analysis the preferred 2D method for analytes present at low concentrations in complex matrices. ${ }^{70,71,85,86,163}$ Despite these advantages, development of a working on-line, comprehensive 2D-LC method is time-consuming, due to the need to optimize all aspects of each individual dimension, including column type, mobile phase selection and compatibility, analysis time, gradient, and flow rate, prior to development of the complete 2D system..$^{73,98,108}$

The improved separation power of 2D-LC itself does not directly lead to identification, but can provide additional, orthogonal information to assist in proper identification of compounds. This is particularly evident when identification based on MS-data might be challenging based on interfering compounds or in instances of 
previously unreported substances. Less lengthy methods with orthogonal properties exist, but they may have their own limitations. Techniques combining ion mobility spectrometry (IMS) with HRMS to collect orthogonal identification information represent are examples of such methods. In IMS, substances are characterized based on the speed at which analyte ions move through an applied electric field and gas atmosphere before reaching the detector. Minute differences in this speed, or drift time, combined with the orthogonal resolving power of HRMS might be able to provide identification of isomeric and chemically related compounds. ${ }^{164}$ However, increased performance with IMS methods, particularly for applications with drugs of abuse, is often a result of improved sample preparation steps such as solid-phase extraction (SPE) or paper spray. ${ }^{165,166}$ These steps require more time and effort for sample preparation prior to analysis and could cause potential sample loss, contamination, or even decrease of ion intensities, which can be avoided through the use of a comprehensive 2D-LC method of separation. Overlapping drift time peaks may also still occur in some instances. These overlaps could be corrected by using a different drift gas, however, when working with unknown compounds, it is not always apparent that such overlap has occurred and that differing IMS conditions are needed during the analysis prior to MS-detection. ${ }^{165}$

In the present study, an RP x RP separation was developed involving an Agilent Poroshell 120 Bonus-RP column in the first dimension and an Ascentis Express biphenyl column in the second dimension. The latter column was chosen due to its ability to separate aromatic compounds, including synthetic cannabinoids, as a result of the $\pi-\pi$ interactions and the influence of analyte shape on interactions between the compound ring structures and the biphenyl moieties of the column stationary phase. ${ }^{167,168}$ 
Resolution of the components of each mix was initially examined on each column separately. The initial 1D-LC separation of CE Mixes 1 and 2 on the Zorbax Eclipse Plus $\mathrm{C}_{18}$ column demonstrated that there was co-elution of multiple compounds in each mix (Figure 12a and 12b). The Bonus-RP column was then investigated for its selectivity toward the compounds in CE Mixes 1 and 2. Figure 13a and 13b show the chromatograms for these two mixes, respectively. This column demonstrated improved separation of the mixture components, indicating that it would be a good selection for the final 2D-LC method.

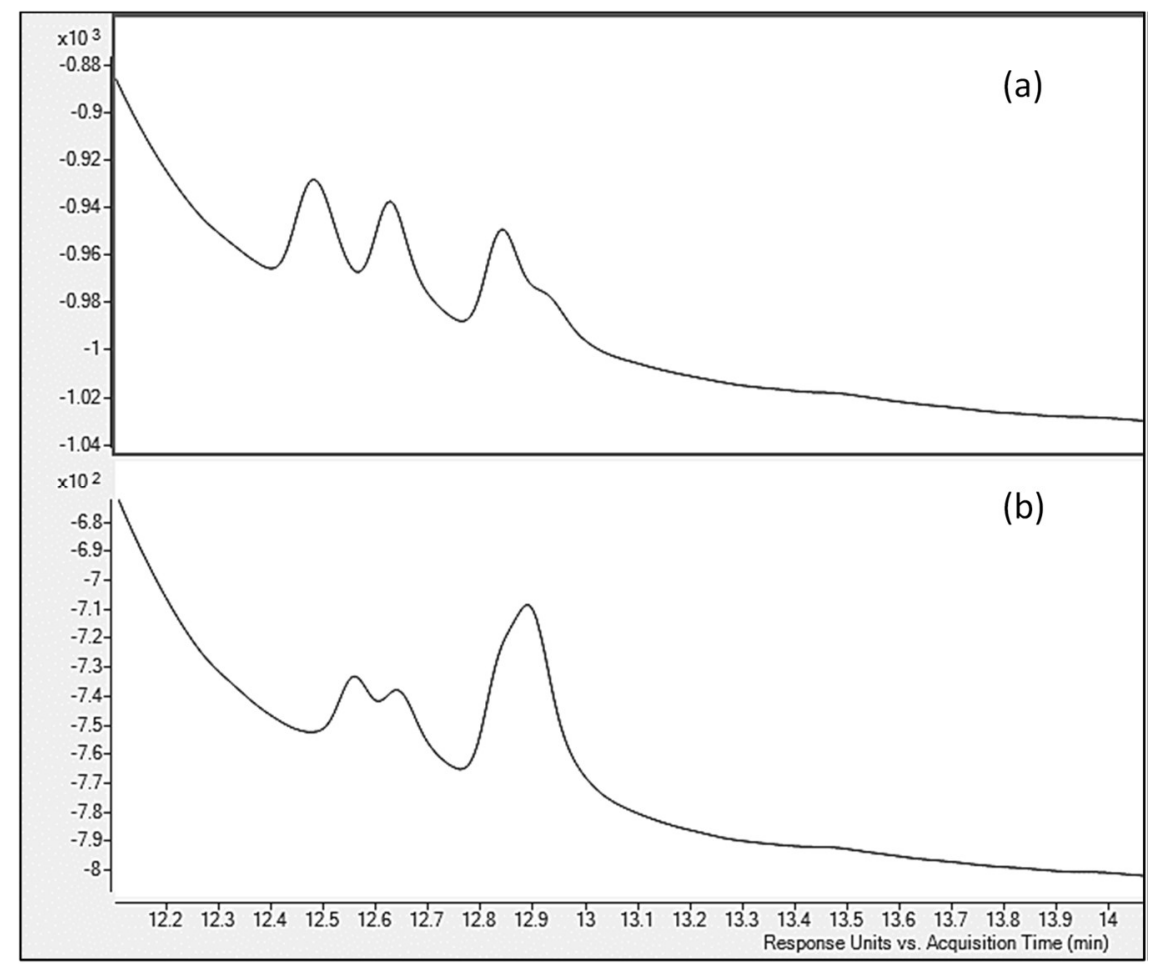

Figure 12. UV chromatograms $(\lambda=220 \mathrm{~nm})$ for $1 \mathrm{D}$ separations of (a) CE Mix 1, and (b) CE Mix 2 with the Zorbax Eclipse Plus $\mathrm{C}_{18}$ column. 
Use of the biphenyl column in 1D-LC separation mode also demonstrated some initial separation of the isomeric compounds in CE Mix 1 (Figure 14a) and the nonisobaric components of CE Mix 2 (Figure 14b). These data suggested that the biphenyl column could be a good choice for use in the final 2D-system in order to achieve full separation and resolution of all mixture components. The Bonus-RP and biphenyl columns were then combined in a 2D-system and tested for separation of CE mixes using diode array and QTOF-MS for detection in the first and second dimensions, respectively. Optimization of the final 2D-LC method based on the results of the 1D-LC experiments was performed to increase its applicability to the wider range of compounds included in the CE mixes, as well as to facilitate coupling of the two dimensions. While the mechanics of a 2D-LC system are only briefly discussed here, further information can be found in recently published works discussing general function of 2D-LC systems. ${ }^{74,102}$

The resulting 2D-LC method was then tested on CE Mixes 1 and 2 (i.e., isomeric and non-isobaric component mixtures, respectively) with diode array detection following the first dimension and QTOF-MS identification following the second. In addition, as a further test, a new non-isobaric component mixture (i.e., CE Mix 3) was formulated. This contained two components from CE Mix 2 (JWH 080 and JWH 203) in addition to three other SC for which very close retention times were also observed in 1D-LC analyses (JWH 073 2'-naphthyl-N-(1,1-dimethylethyl) isomer, JWH 019 N-(2fluorohexyl) isomer, and FUB-144). CE Mixes 1 and 2 were used for initial testing of the 2D-LC method. CE Mix 3 was later created containing components from CE Mixes 1 and 2, in addition to other non-isobaric compounds, as a final test of the method. 2D-LC of CE Mix 1 resulted in excellent separation of the JWH-019 2-, 5-, and 6-fluorohexyl 
isomers with slight co-elution of the 3- and 4-fluorohexyl isomers (Figure 15a). 2D-LC of CE Mix 2 resulted in complete separation of PB-22, JWH 203, and XLR-12, with slight co-elution of MAM-2201 N-(2-fluoropentyl) isomer and JWH 080 (Figure 15b). Finally, 2D-LC separation of CE Mix 3 yielded complete resolution of all five cannabinoid components (Figure 15c).

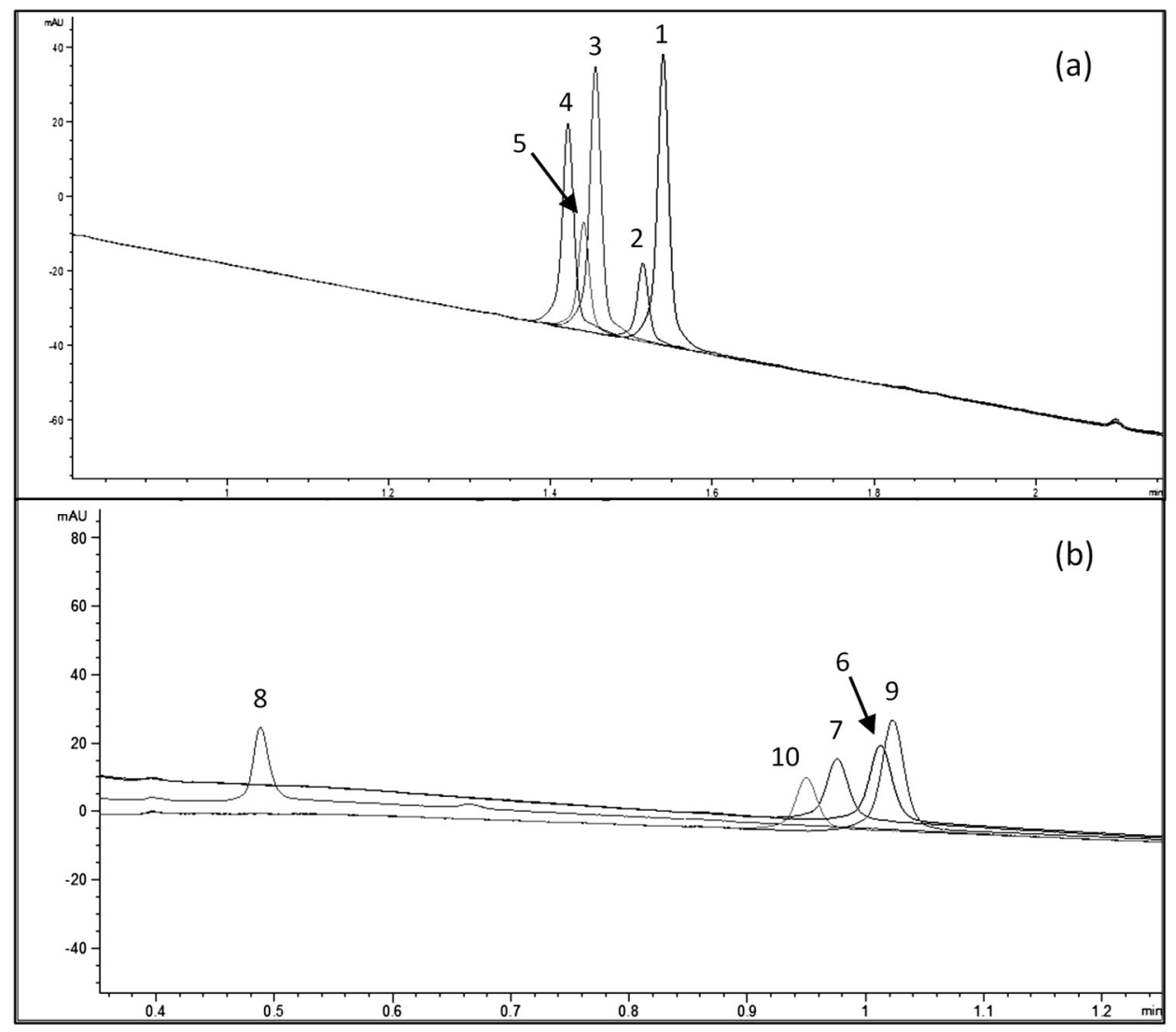

Figure 13. UV chromatograms ( $\lambda=240 \mathrm{~nm}$ ) for 1D separations of (a) CE Mix 1, and (b) CE Mix 2 using the Bonus-RP column over $3 \mathrm{~min}$ at a flow rate of $0.5 \mathrm{~mL} / \mathrm{min}$ with a gradient of $50-95 \% \mathrm{~B}$; A: water $+0.1 \%$ TFA, and B: acetonitrile. Peak numbers correspond to compounds listed in Table 21.

The present research focused on the initial development of an on-line, comprehensive 2D-LC method for the separation of synthetic cannabinoids using a reversed-phase (RP) column in each dimension. 2D-LC involving RP columns has been employed with pharmaceuticals and other small molecules, suggesting their potential 
utility for separation of NPS including SC, ${ }^{85,94,97,108,124}$ Two detectors were used for method development; a diode array detector after the first dimension and a quadrupole time-of-flight (QTOF) mass spectrometer after the second dimension. In a final method, the first detector would be unnecessary, as the ${ }^{2} \mathrm{D}$ MS detector provides accurate mass data of the separated compounds for identification within $\pm 5 \mathrm{ppm}$.

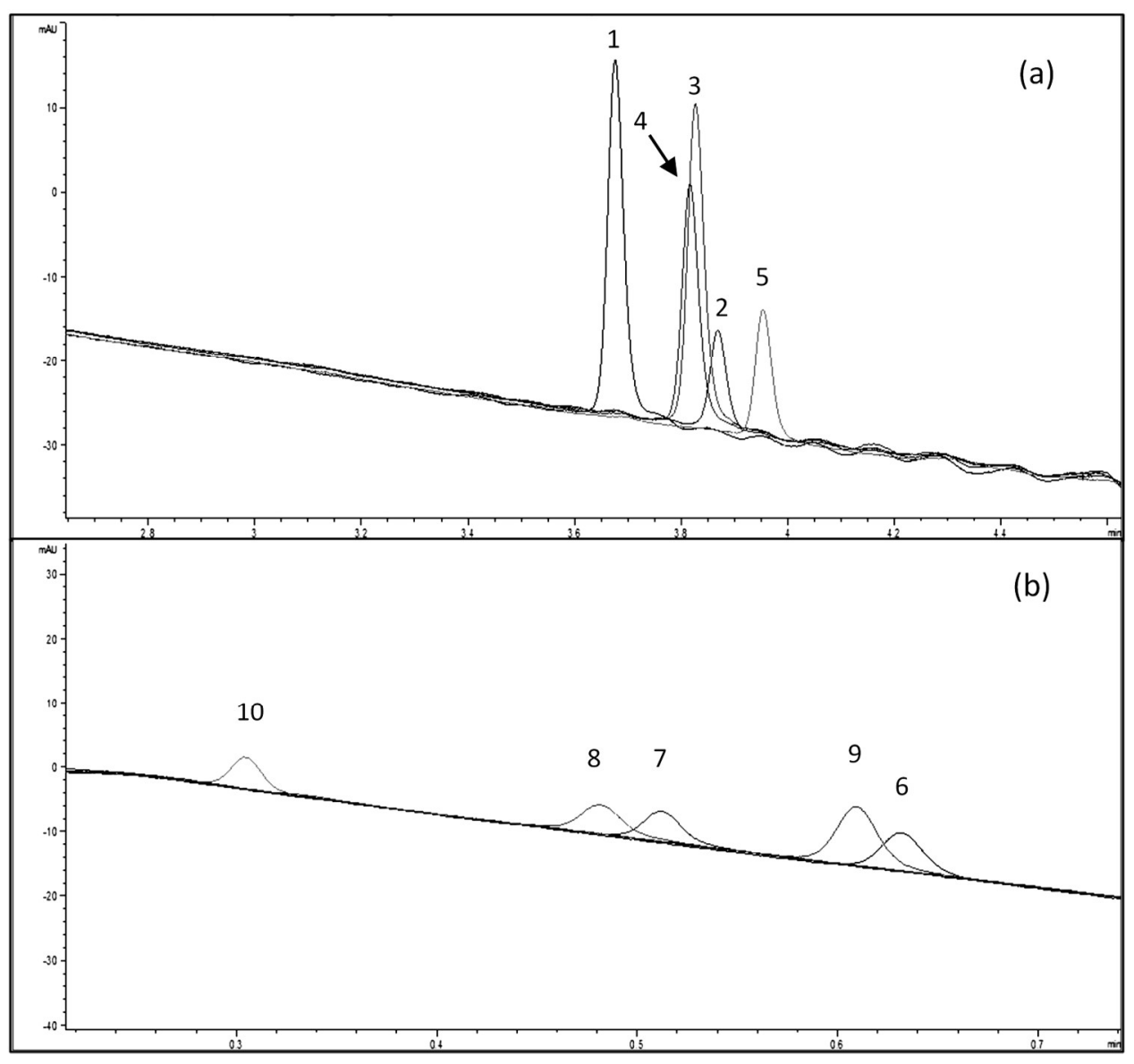

Figure 14. UV chromatograms ( $\lambda=240 \mathrm{~nm}$ ) for $1 \mathrm{D}$ separation of (a) CE Mix 1 over 5 min, and (b) CE Mix 2 over $3 \mathrm{~min}$ using the biphenyl column at a flow rate of $0.5 \mathrm{~mL} / \mathrm{min}$ with a gradient of $70-95 \% \mathrm{~B}$; A: water $+0.1 \%$ TFA, and B: MeOH. Peak numbers correspond to compounds listed in Table 21 .

The Bonus-RP column was ultimately selected for use in ${ }^{1} \mathrm{D}$ in part due to its column packing which has a polar amide group in the long alkyl chain, making it a good choice for use with basic analytes, generally resulting in good peak shape, as well as the 
different separation selectivities it demonstrated as compared to standard $\mathrm{C}_{18}$ columns, including the one initially tested in the present study. ${ }^{169,170}$ The stationary phase in the biphenyl column used in the second dimension is quite different, with a biphenyl moiety bonded to the silica particle. This chemistry provides selectivity that is complementary to typical $\mathrm{C}_{18} \mathrm{RP}$ columns, which is useful in the context of a 2D-LC method. Consequently, a biphenyl column can be an ideal choice for use with $\mathrm{SC}$, due to the greater affinity for the aromatic groups and alkyl chains present in many of these compounds. $^{167,168,171}$

\subsection{Conclusion}

The present report is the first to describe a comprehensive, on-line twodimensional liquid chromatography (RP x RP) method that would be a suitable technique for the separation of NPS. Results of the study serve as a proof-of-concept for the application of $2 \mathrm{D}-\mathrm{LC}$ to the separation of isomeric and structurally related $\mathrm{SC}$. We believe that with further investigation, optimization, and validation, $2 \mathrm{D}-\mathrm{LC}$ will be a viable tool for more reliable separation of complex mixtures of SC compared to what can currently be achieved using conventional 1D-LC. 


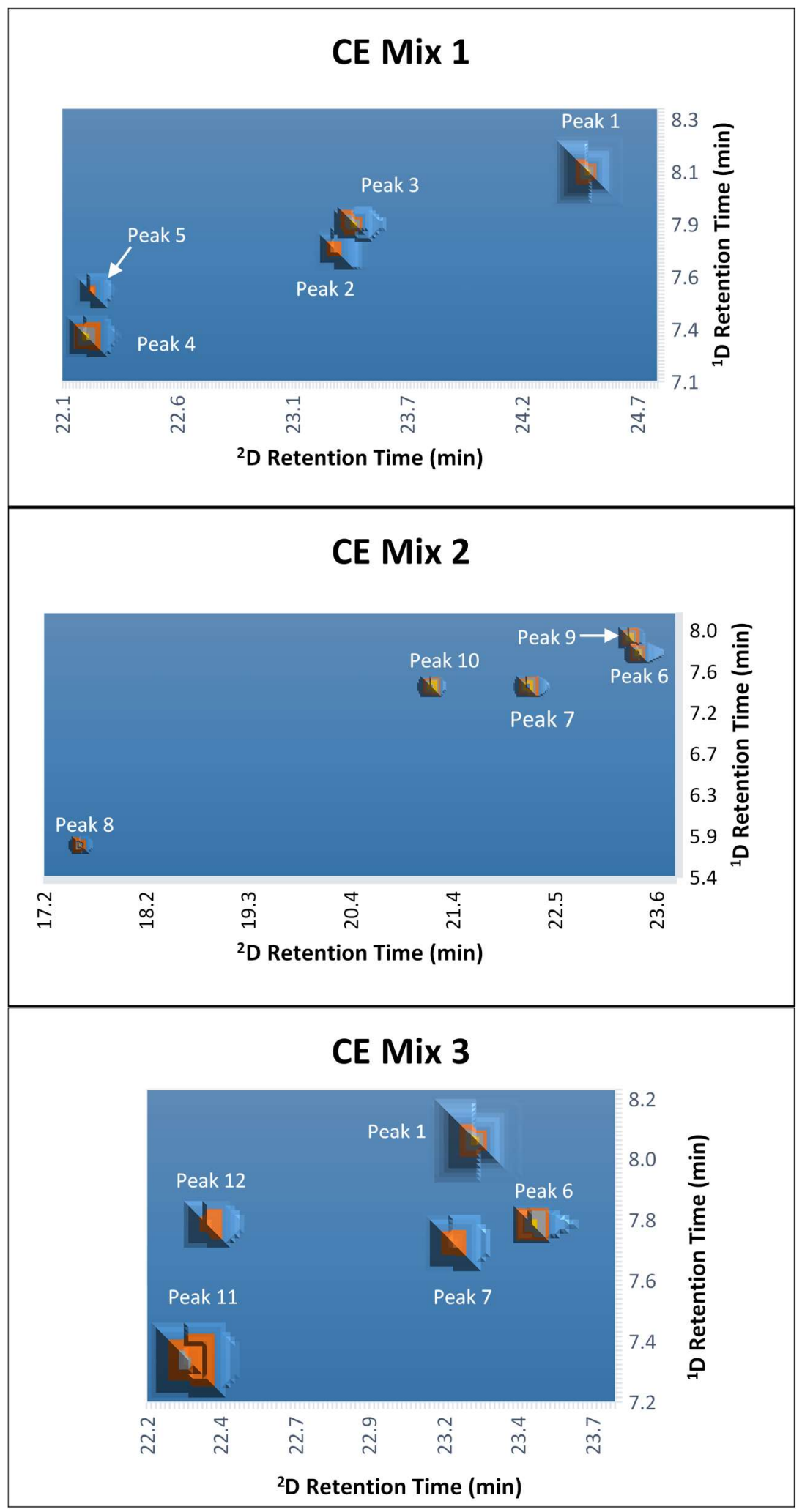

Figure 15. Contour plots demonstrating the 2D separation of CE mixes 1, 2, and 3. Peak number correspond to those listed in Table 21. 


\section{SUMMARY AND PROSPECT}

The constant emergence of novel psychoactive substances will continue to present new analytical challenges to both forensic and clinical toxicology laboratories. Current screening methods typically rely on screening methods designed for specific compounds or compound classes which have demonstrated inconsistent, and often lacking, applicability to specimens containing NPS. Other methods of screening, such as the use of accurate mass databases and MS/MS libraries, present another option for more comprehensive, general screening and confirmation of compounds in human specimens. However, these tools are lacking in the presence of many NPS and remedying such deficiencies requires resources to which some laboratories do not have access (i.e., poor availability of reference standards). The ability to identify these substances in forensic and clinical human specimens is important for conducting comprehensive toxicological screening and confirmation. It was this project's aim to create a large high resolution MS/MS spectral library and compound database for several hundreds of novel psychoactive substances and related compounds in order to aid the forensic and clinical toxicological communities in detecting and identifying such substances in human specimens. A method for comprehensive screening and confirmation was also validated for use with the HRMS spectral library and compound database using a mixture approach.

Mass spectral techniques have gained favor in toxicological laboratories for screening purposes due to their capability of collecting data with high degrees of selectivity and sensitivity which greatly aid in identification substances in toxicological samples. These techniques also have an advantage over other methods of screening, such 
as immunoassays, in that they are not designed to detect a specific compound or compound class, but rather can be applied to a much broader range of potential analytes. Spectral libraries and compound databases are used in conjunction with mass spectrometry techniques and can be extremely useful for screening purposes. In cases where a reference standard is not available to a laboratory, these resources can be searched and can present a tentative identification based on information contained within the database. When data are collected using high resolution mass spectrometry techniques, such as the LC-QTOF-MS used in this research, the selectivity is greatly increased thus improving confidence in correct identification. This high resolution and high mass accuracy enables differentiation between compounds with very similar accurate masses. The characteristic MS/MS spectral data collected using HRMS techniques also increases confidence in identification based on fragmentation patterns of each analyte. HRMS data collection can include information for all ions present in the sample which can then be retrospectively searched when new NPS are reported without the need for sample reanalysis. The use of the compound database and MS/MS spectral library created by this work can greatly assist forensic and clinical toxicological laboratories in saving valuable time and resources when attempting to identify novel psychoactive substances in toxicological specimens.

Following completion of the compound database and MS/MS library, a comprehensive method for screening and confirmation was validated following standard guidelines from the Toxicology Subcommittee of the Organization of Scientific Area Committees. Proof of concept was achieved for the use of a mixture approach for validation as opposed to validating the method for a single compound or class of 
compounds one at a time. This technique saved valuable time and resources, and also demonstrated that the method could be applied comprehensively to a broad range of NPS from a variety of compound classes. The mixture approach has shown that it can be used in the future by other laboratories when validating toxicological screening and confirmation methods for a large group of NPS. The method was successfully validated for detection and identification of NPS at low $\mathrm{ng} / \mathrm{mL}$ concentrations. Applicability of the method with the database and HRMS spectral library was also demonstrated through the qualitative screening of blind-spiked human urine specimens.

Finally, throughout the creation of the compound database and MS/MS spectral library, and during the subsequent method validation, it became apparent that some compounds could not be differentiated based on MS/MS data or chromatographically. This was especially apparent with isomeric compounds and those with related chemical structures. Typically, HRMS methods may be used to determine compounds with highly similar accurate masses, however, in instances of unreported and truly unknown NPS, it would be difficult to determine if a collected mass spectrum was representative of a single analyte or of multiple ones. In the case of isomers which have the same or similar MS/MS spectra, chromatographic information can be used. However, if adequate separation of the compounds cannot be achieved through traditional chromatographic methods, a different approach must be used. Two-dimensional liquid-chromatography (2D-LC) was investigated as part of this project to determine if it could be a viable option for separation of co-eluting compounds; mainly isomers and structurally similar compounds. 
Mixtures of synthetic cannabinoids — both isomeric and non-isobaric — were created for the 2D-LC investigations. An on-line, comprehensive method using a Bonus$\mathrm{RP}$ column in the first dimension and a biphenyl column in the second dimension was developed as a proof-of-concept for the application of 2D-LC to separation of such mixtures. Separation was successfully achieved for all compounds present in each mixture, but further development will be required in order to broaden applicability of the technique to NPS from other compound classes, as separation parameters can be compound specific.

A large compound database and MS/MS spectral library was successfully created and a corresponding method for screening and confirmation was fully validated using a mixture approach. These resources present valuable tools for forensic and clinical toxicology laboratories to use when screening for a wide variety of NPS. A twodimensional liquid chromatographic method for separation of synthetic cannabinoids was also developed and demonstrated successful separation of isomeric and non-isobaric compounds from that class.

Future work will be required, however, to update and expand the compound database and MS/MS spectral library as more NPS are reported and as appropriate reference standards become commercially available. HRMS techniques should continue to gain favor for toxicological applications as they demonstrate improved selectivity and sensitivity over other screening approaches. Further investigation of two-dimensional liquid chromatography for improved separation of isomeric and structurally related NPS should also be conducted, as complete separation using traditional methods is likely to be challenged as more compounds emerge on the market. 


\section{REFERENCES}

1. Behonick, G.; Shanks, K. G.; Firchau, D. J.; Mathur, G.; Lynch, C. F.; Nashelsky, M. Four postmertem case reports with quantitative detection of the synthetic cannabinoid, 5F-PB-22. Journal of Analytical Toxicology 2014, 38, 559-562.

2. deRoux, S. J.; Dunn, W. A. "Bath salts" the New York City Medical Examiner experience: A 3-year retrospective review. Journal of Forensic Sciences 2017, 62, 695-699.

3. Shanks, K. G.; Sozio, T.; Behonick, G. S. Fatal intoxications with 25B-NBOMe and 25I-NBOMe in Indiana during 2014. Journal of Analytical Toxicology 2015, 39, $602-606$.

4. Lee, D.; Chronister, C. W.; Broussard, W. A.; Utley-Bobak, S. R.; Schultz, D. L.; Vega, R. S. Illicit fentanyl-related fatalities in Florida: toxicological findings. Journal of Analytical Toxicology 2016, 40, 588-594.

5. Henderson, G. L. Designer drugs: past history and future prospects. Journal of Forensic Sciences 1988, 33, 569-575.

6. Swortwood, M. J.; Hearn, W. L.; DeCaprio, A. P. Cross-reactivity of designer drugs, including cathinone derivatives, in commercial enzyme-linked immunosorbent assays. Drug Testing and Analysis 2014, 6, 716-727.

7. Broecker, S.; Herre, S.; Wüst, B.; Zweigenbaum, J.; Pragst, F. Development and practical application of a library of CID accurate mass specra of more than 2,500 toxic compounds for systematic toxicological analysis by LC-QTOF-MS with datadependant acquisition. Analytical and Bioanalytical Chemistry 2011, 400, 101-117.

8. Peters, F. T. Recent advances of liquid chromatography-(tandem) mass spectrometry in clinical and forensic toxicology. Clinical Biochemistry 2011, 44, 54-65.

9. Rosano, T. G.; Wood, M.; Ihenetu, K.; Swift, T. A. Drug screening in medical examiner casework by high-resolution mass spectrometry (UPLC-MS ${ }^{\mathrm{E}}$-TOF). Journal of Analytical Toxicology 2013, 37, 593.

10. Logan, B. K.; Mohr, A. L. A.; Krotulski, A. J.; Papsun, D. M.; Kacinko, S. L.; Ropero-Miller, J. D.; Huestis, M. A. Reports of adverse events associated with use of novel psychoactive substances, 2013-2016: A review. Journal of Analytical Toxicology 2017, 41, 573-610.

11. Lua, I. A.; Lin, S.-L.; Lin, H. R.; Lua, A. C. Replacing immunoassays for mephedrone, ketamines, and six amphetamine-type stimulants with flow injection 
analysis tandem mass spectrometry. Journal of Analytical Toxicology 2012, 36, 575-581.

12. Nakanishi, K.; Miki, A.; Zaitsu, K.; Kamata, H.; Shima, N.; Kamata, T.; Katagi, M.; Tatsuno, M.; Tsuchihashi, H.; Suzuki, K. Cross-reactivities of various phenethylamine-type designer drugs to immunoassays for amphetamines, with special attention to the evaluation of the one-step urine drug test Instant-View ${ }^{\mathrm{TM}}$, and the Emit ${ }^{\circledR}$ assays for use in drug enforcement. Forensic Science International 2012, 217, 174-181.

13. DEA Diversion Control Division . Controlled Substance Schedules. 2018.

14. Title 21 United States Code (U.S.C.) Controlled Substances Act. U. S. Department of Justice, Drug Enforcement Administration Diversion Control Division 2018.

15. Carroll, F. I.; Lewin, A. H.; Mascarella, S. W.; Seltzman, H. H.; Reddy, P. A. Designer drugs: a medicinal chemistry perspective. Annals of the New York Academy of Sciences 2012, 1248, 18-38.

16. Boumba, V. A.; Di Rago, M.; Peka, M.; Drummer, O. H.; Gerostamoulos, D. The analysis of 132 novel psychoactive substances in human hair using a single extraction by tandem LC/MS. Forensic Science International 2017, 279, 192-202.

17. Hill, S. L.; Thomas, S. H. L. Clinical toxicology of newer recreational drugs. Clinical Toxicology 2011, 49, 705-719.

18. Grabenauer, M.; Krol, W. L.; Wiley, J. L.; Thomas, B. F. Analysis of synthetic cannabinoids using high-resolution mass spectrometry and mass defect filtering: implications for nontargeted screening of designer drugs. Analytical Chemistry 2012, $84,5574-5581$.

19. Griffiths, P.; Sedefov, R.; Gallegos, A.; Lopez, D. How globalization and market innovation challenge how we think and respond to drug use: 'Spice" a case study. Addiction 2010, 105, 951-953.

20. Donohue, K. M.; Steiner, R. R. JWH-018 and JWH-022 as Combustion Products of AM2201. Microgram Journal 2012, 9, 52-56.

21. Finley, S. A. Two Additional Synthetic Drug Traffickers Plead Guilty in Curious Goods Case. The United States Department of Justice. 2012.

22. DEA Diversion Control Division 4-Methylmethcathinone (Mephedrone); 13.

23. Drug Enforcement Administration . DEA Scheduling Actions. 2014. 11-9-2014.

24. Nieddu, M.; Burrai, L.; Baralla, E.; Pasciu, V.; Varoni, M. V.; Briguglio, I.; Demontis, M. P.; Boatto, G. ELISA detection of 30 new amphetamine designer 
drugs in whole blood, urine and oral fluid using Neogen ${ }^{\circ}$ "Amphetamine" and "Methamphetamine/MDMA" kits. Journal of Analytical Toxicology 2016, 40, 492497.

25. Scheidweiler, K. B.; Jarvis, M. J. Y.; Huestis, M. A. Nontargeted SWATH acquisition for identifying 47 synthetic cannabinoid metabolites in human urine by liquid chromatography-high-resolution tandem mass spectrometry. Analytical and Bioanalytical Chemistry 2015, 407, 883-897.

26. Sundström, M.; Pelander, A.; Angerer, V.; Hutter, M.; Kneisel, S.; Ojanperä, I. A high-sensitivity ultra-high performance liquid chromatography/high-resolution time-of-flight mass spectrometry (UHPLC-HR-TOFMS) method for screening synthetic cannabinoids and other drugs of abuse in urine. Analytical and Bioanalytical Chemistry 2013, 405, 8463-8474.

27. Ojanpera, I.; Pelander, A.; Laks, S.; Gergov, M.; Vuori, E.; Witt, M. Application of Accurate Mass Measurement to Urine Drug Screening. Journal of Analytical Toxicology 2005, 29, 34-40.

28. Ojanperä, I.; Pelander, A.; Ojanperä, S. Comprehensive toxicological and forensic drug screening by LC/QTOF-MS. Ferrer, I., Thurman, E. M., Eds.; 2009; pp 173195.

29. Andersson, M.; Gustavsson, E.; Stephanson, N.; Beck, O. Direct injection LCMS/MS method for identification and quantification of amphetamine, methamphetamine, 3,4-methylenedioxyamphetamine and 3,4methylenedioxymethamphetamine in urine drug testing. Journal of Chromatography B 2008, 861, 22-28.

30. Svensson, J.-O.; Andersson, M.; Gustavsson, E.; Beck, O. Electrospray LC-MS method with solid-phase extraction for accurate determination of morphine-, codeine-, and ehtylmorphine-glucuronides and 6-acetylmorphine in urine. Journal of Analytical Toxicology 2007, 31, 81-86.

31. Gustavsson, E.; Andersson, M.; Stephanson, N.; Beck, O. Validation of direct injection electrospray LC-MS/MS for confirmation of opiates in urine drug testing. Journal of Mass Spectrometry 2007, 42, 881-889.

32. Politi, L.; Morini, L.; Polettini, A. A direct screening procedure for diuretics in human urine by liquid chromatography-tandem mass spectrometry with information dependent acquisition. Clinica Chimica Acta 2007, 386, 46-52.

33. Nieddu, M.; Trignano, C.; Burrai, L.; Pirisi, M. A.; Boatto, G. Cross-reactivities of 41 new amphetamine designer drugs to EMIT ${ }^{\circledR}$ immunoassays. Forensic Toxicology 2013, 31, 133-137. 
34. Mueller, C. A.; Weinmann, W.; Dresen, S.; Schreiber, A.; Gergov, M. Development of a multi-target screening analysis for 301 drugs using QTrap liquid chromatography/tandem mass spectrometry system and automated library searching. Rapid Communications in Mass Spectrometry 2005, 19, 1332-1338.

35. Substance Abuse and Mental Health Services Administration . Drug Testing. 2015.

36. Decaestecker, T. N.; Vande Casteele, S. R.; Wallemacq, P. E.; Van Peteghem, C. H.; Defore, D. L.; Van Bocxlaer, J. F. Information-dependent acquisition-mediated LC-MS/MS screening procedure with semiquantitative potential. Analytical Chemistry 2004, 76, 6365-6373.

37. Concheiro, M.; Castaneto, M.; Kronstrand, R.; Huestis, M. A. Simultaneous determination of 40 novel psychoactive substances in urine by liquid chromatography-high resolution mass spectrometry and library matching. Journal of Chromatography A 2015, 1397, 32-42.

38. Dresen, S.; Ferreirós, N.; Gnann, H.; Zimmermann, R.; Weinmann, W. Detection and identification of 700 drugs by multi-target screening with a 3200 Q TRAP ${ }^{\circledR}$ LC-MS/MS system and library searching. Analytical and Bioanalytical Chemistry 2010, 396, 2425-2434.

39. Kerrigan, S.; Mellon, M. B.; Banuelos, S.; Arndt, C. Evaluation of commercial enzyme-linked immunosorbent assays to identify psychedelic phenethylamines. Journal of Analytical Toxicology 2011, 35, 444-451.

40. Meyer, M. R.; Maurer Hans H. Review: LC coupled to low- and high-resolution mass spectrometry for new psychoactive substance screening in biological matrices - Where do we stand today? Analytica Chimica Acta 2016, 927, 13-20.

41. Petrie, M.; Lynch, K. L.; Ekins, S.; Chang, J. S.; Goetz, R. J.; Wu, A. H. B.; Krasowski, M. D. Cross-reactivity studies and predicitve modeling of "Bath Salts" and other amphetamine-type stimulants with amphetamine screening immunoassays. Clinical Toxicology 2013, 51, 83-91.

42. Stein, S. Mass spectral reference libraries: an ever-expanding resource for chemical identification. Analytical Chemistry 2012, 84, 7274-7284.

43. Stein, S. E. Chemical structure identification by mass spectral library searching. Journal of the American Society for Mass Spectrometry 1995, 6, 644-655.

44. Moorthy, A. S.; Wallace, W. E.; Kearsley, A. J.; Tchekhovskoi, D. V.; Stein, S. E. Combining fragment-ion and neutral-loss matching during mass spectral library searching: A new general purpose algorithm applicable to illicit drug identification. Analytical Chemistry 2017, 89, 13261-13268. 
45. Alechaga, É.; Moyano, E.; Galceran, M. T. Wide-range screening of psychoactive substances by FIA-HRMS: identification strategies. Analytical and Bioanalytical Chemistry 2015, 407, 4567-4580.

46. Partridge, E.; Trobbiani, S.; Stockham, P.; Scott, T.; Kostakis, C. A validated method for the screening of 320 forensically significant compounds in blood by LC/QTOF, with simultaneous quantification of selected compounds. Journal of Analytical Toxicology 2018, 42, 220-231.

47. Pavlic, M.; Libiseller, K.; Oberacher, H. Combined use of ESI-QqTOF-MS and ESI-QqTOF-MS/MS with mass-spectral library search for qualitative analysis of drugs. Analytical and Bioanalytical Chemistry 2006, 386, 69-82.

48. Smith, R. M. Understanding Mass Spectra: A Basic Approach; John Wiley \& Sons, Inc.: New York, NY, 1999.

49. NIST 17 Mass Spectral Library \& Search Software Version 2017 Brochure. 2017.

50. Lee, H. K.; Ho, C. S.; Iu, Y. P. H.; Lai, P. S. J.; Shek, C. C.; Lo, Y.-C.; Klinke, H. B.; Wood, M. Development of a broad toxicological screening technique for urine using ultra-performance liquid chromatography and time-of-flight mass spectrometry. Analytica Chimica Acta 2009, 649, 80-90.

51. Lynch, K. L.; Breaud, A. R.; Vandenberghe, H.; Wu, A. H. B.; Clarke, W. Performance evaluation of three liquid chromatography mass spectrometry methods for broad spectrum drug screening. Clinica Chimica Acta 2010, 411, 1474-1481.

52. de Hoffmann, E.; Stroobant, V. Mass Spectrometry: Principles and Applications; 3rd ed.; John Wiley \& Sons, Ltd.: 2007.

53. Dresen, S.; Gergov, M.; Politi, L.; Halter, C.; Weinmann, W. ESI-MS/MS library of 1,253 compounds for application in forensic and clinical toxicology. Analytical and Bioanalytical Chemistry 2009, 395, 2521-2526.

54. Namera, A.; Kawamura, M.; Nakamoto, A.; Saito, T.; Nagao, M. Comprehensive review of the detection methods for synthetic cannabinoids and cathinones. Forensic Toxicology 2015, 33, 175-194.

55. Yanes, E. G.; Lovett, D. P. High-throughput bioanalytical method for analysis of synthetic cannabinoid metabolites in urine using salting-out sample preparation and LC-MS/MS. Journal of Chromatography B 2012, 909, 42-50.

56. Dresen, S.; Kempf, J.; Weinmann, W. Electrospray-ionization MS/MS library of drugs as database for method development and drug identification. Forensic Science International 2006, 161, 86-91. 
57. Weinmann, W.; Gergov, M.; Goerner, G. MS/MS-libraries with triple quadrupoletandem mass spectrometers for drug identification and drug screening. Analusis 2000, 28,941 .

58. Oberacher, H.; Pavlic, M.; Libiseller, K.; Schubert, B.; Sulyok, M.; Schuhmacher, R.; Csaszar, E.; Köfeler, H. C. On the inter-instrument and the inter-laboratory transferability of a tandem mass spectral reference library: 1. Results of an Austrian multicenter study. Journal of Mass Spectrometry 2009, 44, 485-493.

59. Broecker, S.; Herre, S.; Pragst, F. General unknown screening in hair by liquid chromatography-hybrid quadrupole time-of-flight mass spectrometry (LC-QTOFMS). Forensic Science International 2012, 218, 68-81.

60. Polettini, A.; Gottardo, R.; Pascali, J. P.; Tagliaro, F. Implementation and performance evaluation of a database of chemical formulas for the screening of pharmaco/toxicologically relevant compounds in biological samples using electrospray ionization-time-of-flight mass spectrometry. Analytical Chemistry 2008, $80,3050-3057$.

61. Ojanperä, S.; Pelander, A.; Pelzing, M.; Krebs, I.; Vuori, E.; Ojanperä, I. Isotopic pattern and accurate mass determination in urine drug screening by liquid chromatography/time-of-flight mass spectrometry. Rapid Communications in Mass Spectrometry 2006, 20, 1161-1167.

62. Pelander, A.; Tyrkkö, E.; Ojanperä, I. In silico methods for predicting metabolism and mass fragmentation applied to quetiapine in liquid chromatography/time-offlight mass spectrometry in urine drug screening. Rapid Communications in Mass Spectrometry 2009, 23, 506-514.

63. Colby, J. M.; Thoren, K. L.; Lynch, K. L. Suspect screening using LC-QqTOF is a useful tool for detecting drugs in biological specimens. Journal of Analytical Toxicology 2018, 42, 207-213.

64. Kinyua, J.; Negreira, N.; Ibáñez, M.; Bijlsma, L.; Hernández, F.; Covaci, A.; van Nuijs, A. L. N. A data-independent acquisition workflow for qualitative screening of new psychoactive substances in biological samples. Analytical and Bioanalytical Chemistry 2015, 407, 8773-8785.

65. Choi, B. K.; Hercules, D. M.; Zhang, T.; Gusev, A. I. Comparison of quadrupole, time-of-flight, and Fourier transform mass analyzers for LC-MS applications. Current Trends in Mass Spectrometry 2003, 18, 24-31.

66. Ojanperä, I.; Kolmonen, M.; Pelander, A. Current use of high-resolution mass spectrometry in drug screening relevant to clinical and forensic toxicology and doping control. Analytical and Bioanalytical Chemistry 2012, 403, 1203-1220. 
67. Broecker, S.; Pragst, F.; Bakdash, A.; Herre, S.; Tsokos, M. Combined use of liquid-chromatography-hybrid quadrupole time-of-flight mass spectrometry (LCQTOF-MS) and high performance liquid chromatography with photodiode array detector (HPLC-DAD) in systematic toxicological analysis. Forensic Science International 2011, 212, 215-226.

68. Muñoz-Muñoz, A. C.; Pekol, T.; Schubring, D.; Johnson, C.; Andrade, L. Identification of novel opioid interferences using high-resolution mass spectrometry. Journal of Analytical Toxicology 2018, 42, 6-16.

69. Ibáñez, M.; Sancho, J. V.; Bijlsma, L.; van Nuijs, A. L. N.; Covaci, A.; Hernández, F. Comprehensive analytical strategies based on high-resolution time-of-flight mass spectrometry to identify new psychoactive substances. Trends in Analytical Chemistry 2014, 57, 107-117.

70. Fairchild, J. N.; Horváth, K.; Guiochon, G. Approaches to comprehensive multidimensional liquid chromatography systems. Journal of Chromatography A 2009, 1216, 1363-1371.

71. Fairchild, J. N.; Horváth, K.; Guiochon, G. Theoretical advantages and drawbacks of on-line, multidimensional liquid chromatography using multiple columns operated in parallel. Journal of Chromatography A 2009, 1216, 6210-6217.

72. Jayamanne, M.; Granelli, I.; Tjernberg, A.; Edlund, P.-O. Development of a twodimensional liquid chromatography system for isolation of drug metabolites. Journal of Pharmaceutical and Biomedical Analysis 2010, 51, 649-657.

73. Pirok, B. W. J.; Gargano, A. F. G.; Schoenmakers, P. J. Optimizing separations in online comprehensive two-dimensional liquid chromatography. Journal of Separation Science 2018, 41, 68-98.

74. Stoll, D. R.; Carr, P. W. Two-dimensional liquid chromatography: a state of the art tutorial. Analytical Chemistry 2017, 89, 519-531.

75. Guiochon, G.; Marchetti, N.; Mriziq, K.; Shalliker, R. A. Implementations of twodimensional liquid chromatography. Journal of Chromatography A 2008, 1189, 109-168.

76. Rao, R. N.; Kumar, K. N.; Shinde, D. D. Determination of rat plasma levels of sertraline enantiomers using direct injection with achiral-chiral column switching by LC-ESI/MS/MS. Journal of Pharmaceutical and Biomedical Analysis 2010, 52, 398-405.

77. Hudson, S.; Ramsey, J. The emergence and analysis of synthetic cannabinoids. Drug Testing and Analysis 2011, 3, 466-478. 
78. Stoll, D. R. Guidelines for bioanalytical 2D chromatography method development and implementation. Bioanalysis 2010, 2, 105-122.

79. Cassiano, N.; Barreiro, J.; Oliveira, R.; Cass, Q. Direct bioanalytical sample injection with 2D LC-MS. Bioanalysis 2012, 4, 2737-2756.

80. Donato, P.; Cacciola, F.; Quinto Tranchida, P.; Dugo, P.; Mondello, L. Mass spectrometry detection in comprehensive liquid chromatography: basic concepts, instrumental aspects, applications and trends. Mass Spectrometry Reviews 2012, 31, 523-559.

81. Mondello, L.; Dugo, P.; Kumm, T.; Cacciola, F.; Dugo, G. Two-dimensional comprehensive liquid chromatography. In Handbook of HPLC, 2 ed.; Corradini, D., Phillips, T. M., Eds.; CRC Press: 2010.

82. Andrighetto, L. M.; Burns, N. K.; Stevenson, P. G.; Pearson, J. R.; Henderson, L. C.; Bowen, C. J.; Conlan, X. A. In-silico optimisation of two-dimensional high performance liquid chromatography for the determination of Australian methamphetamine seizure samples. Forensic Science International 2016, 266, 511516 .

83. Opiteck, G. J.; Lewis, K. C.; Jorgenson, J. W.; Anderegg, R. J. Comprehensive online LC/LC/MS of proteins. Analytical Chemistry 1997, 69, 1518-1524.

84. Opiteck, G. J.; Ramirez, S. M.; Jorgenson, J. W.; Moseley, M. A. Comprehensive two-dimensional high performance liquid chromatography for the isolation of overexpressed proteins and proteome mapping. Analytical Biochemistry 1998, 258, 349-361.

85. Malerod, H.; Lundanes, E.; Greibrokk, T. Recent advances in on-line multidimensional liquid chromatography. Analytical Methods 2010, 2, 110-122.

86. Agilent Technologies, I. Agilent 1290 Infinity 2D-LC-Solution: User Guide; 2012.

87. Bedani, F.; Schoenmakers, P. J.; Janssen, H.-G. Theories to support method development in comprehensive two-dimensional liquid chromatography - A review. Journal of Separation Science 2012, 35, 1697-1711.

88. Fairchild, J. N.; Horváth, K.; Gooding, J. R.; Campagna, S. R.; Guiochon, G. Twodimensional liquid chromatography/mass spectrometry/mass spectrometry separation of water-soluble metabolites. Journal of Chromatography A 2010, 1217, 8161-8166.

89. Rao, R. N.; Shinde, D. D. Two-dimensional LC-MS/MS determination of antiretroviral drugs in rat serum and urine. Journal of Pharmaceutical and Biomedical Analysis 2009, 50, 994-999. 
90. Jourdil, J.-F.; Tonini, J.; Stanke-Labesque, F. Simultaneous quantitation of azole antifungals, antibiotics, imatinib, and raltegravir in human plasma by twodimensional high-performance liquic chromatography-tandem mass spectrometry. Journal of Chromatography B 2013, 919-920, 1-9.

91. Mella, M.; Schweitzer, B.; Mallet, C.; Moore, T.; Botch-Jones, S. Detection of cocaine and metabolites in bone following decomposition using 2D LC-MS-MS. Journal of Analytical Toxicology 2018, 42, 265-275.

92. Mallet, C.; Botch-Jones, S. Illicit drug analysis using two-dimension liquid chromatography/tandem mass spectrometry. Journal of Analytical Toxicology 2016, 40, 617-627.

93. Ing-Lorenzini, K. R.; Desmeaules, J. A.; Besson, M.; Veuthey, J.-L.; Dayer, P.; Daali, Y. Two-dimensional liquid-chromatography-ion trap mass spectrometry for the simultaneous determination of ketorolac enantiomers and paracetamol in human plasma: application to a pharmacokinetic study. Journal of Chromatography A 2009, 1216, 3851-3856.

94. Li, Y.; Gu, C.; Gruenhagen, J.; Zhang, K.; Yehl, P.; Chetwyn, N. P.; Medley, C. D. A size exclusion-reveresed phase two dimensional-liquid chromatography methodology for stability and small molecule related species in antibody drug conjugates. Journal of Chromatography A 2015, 1393, 81-88.

95. Pascoe, R.; Foley, J. P.; Gusev, A. I. Reduction in matrix-related signal suppression effects in electrospray ionization mass spectrometry using on-line two-dimensional liquid chromatography. Analytical Chemistry 2001, 73, 6014-6023.

96. Joseph, S.; Subramanian, M.; Khera, S. Simultaneous and stereospecific analysis of warfarin oxidative metabolism using 2D LC/Q-TOF. Bioanalysis 2015, 7, 2297 2309.

97. Sandra, P.; Vanhoenacker, G.; Steenbeke, M.; David, F.; Sandra, K.; Brunelli, C.; Szucs, R. On-line two-dimensional liquid chromatography (2D-LC) for the analysis of pharmaceuticals. LC-GC North America 2016, 29, 610-617.

98. Stoll, D. Recent advances in 2D-LC for bioanalysis. Bioanalysis 2015, 7, 3125 3142 .

99. Pandohee, J.; Holland, B. J.; Li, B.; Tsuzuki, T.; Stevenson, P. G.; Barnett, N. W.; Pearson, J. R.; Jones, O. A. H.; Conlan, X. A. Screening of cannabinoids in industrial-grade hemp using two-dimensional liquid chromatography coupled with acidic potassium permanganate chemiluminescence detection. Journal of Separation Science 2015, 38, 2024-2032. 
100. Agilent Technologies, I. Performance evaluation of the Agilent 1290 Infinity 2DLC Solution for comprehensive two-dimensional liquid chromatography: Technical Overview. 2012.

101. Regalado, E. L.; Schariter, J. A.; Welch, C. J. Investigation of two-dimensional high performance liquid chromatography approaches for reversed phase resolution of warfarin and hydroxywarfarin isomers. Journal of Chromatography A 2014, 1363, 200-206.

102. Stoll, D. Introduction to Two-Dimensional Liquid Chromatography - Theory and Practice. In Handbook of Advanced Chromatography/Mass Spectrometry Techniques, Holcapek, M., Byrdwell, W. C., Eds.; Elsevier: London, 2017; pp 227286.

103. Stoll, D. R.; Li, X.; Wang, X.; Carr, P. W.; Porter, S. E. G.; Rutan, S. C. Fast, comprehensive two-dimensional liquid chromatography. Journal of Chromatography A 2007, 1168, 3-43.

104. Bedani, F.; Kok, W. Th.; Janssen, H.-G. Optimal gradient operation in comprehensive liquid chromatography $\mathrm{x}$ liquid chromatography systems with limited orthogonality. Analytica Chimica Acta 2009, 654, 77-84.

105. Krieger, S. Authentication of traditional Chinese prescriptions using comprehensive 2D-LC. LC-GC North America 2015, 33, 18.

106. François, I.; Sandra, K.; Sandra, P. Comprehensive liquid chromatography: fundamental aspects and practical considerations--a review. Analytica Chimica Acta 2009, 641, 14-31.

107. Chen, X.; Kong, L.; Su, X.; Fu, H.; Ni, J.; Zhao, R.; Zou, H. Separation and identification of compounds in Rhizoma chuanxiong by comprehensive twodimensional liquid chromatography coupled to mass spectrometry. Journal of Chromatography A 2004, 1040, 169-178.

108. Li, D.; Jakob, C.; Schmitz, O. Practical considerations in comprehensive twodimensional liquid chromatography systems (LCxLC) with reversed-phase in both dimensions. Analytical and Bioanalytical Chemistry 2015, 407, 153-167.

109. Zhang, J.; Tao, D.; Duan, J.; Liang, Z.; Zhang, W.; Zhang, L.; Huo, Y.; Zhang, Y. Separation and identification of compounds in Adinandra nitida by comprehensive two-dimensional liquid chromatography coupled to atmospheric pressure chemical ionization source ion trap tandem mass spectrometry. Analytical and Bioanalytical Chemistry 2006, 386, 586-593.

110. Holcapek, M.; Ovcacíková, M.; Lísa, M.; Cífková, E.; Hájek, T. Continuous comprehensive two-dimensional liquid chromatography-electrospray ionization 
mass spectrometry of complex lipidomic samples. Analytical and Bioanalytical Chemistry 2015, 407, 5033-5043.

111. Yang, P.; Pursch, M. Recent advances in two-dimensional liquid chromatography. Chromatography Today 2017, 24-29.

112. Gu; Haiwei; Huang, Y.; Carr, P. W. Peak capacity optimization in comprehensive two dimensional liquid chromatography: A practical approach. Journal of Chromatography A 2011, 1218, 64-73.

113. Dugo, P.; Cacciola, F.; Kumm, T.; Dugo, G.; Mondello, L. Comprehensive multidimensional liquid chromatography: Theory and applications. Journal of Chromatography A 2008, 1184, 353-368.

114. Cao, J.-L.; Wei, J.-C.; Chen, M.-W.; Su, H.-X.; Wan, J.-B.; Wang, Y.-T.; Li, P. Application of two-dimensional chromatography in the analysis of Chinese herbal medicines. Journal of Chromatography A 2014, 1371, 1-14.

115. Hu, L.; Chen, X.; Kong, L.; Su, X.; Ye, M.; Zou, H. Improved performance of comprehensive two-dimensional HPLC separation of traditional Chinese medicines by using a silica monolithic column and normalization of peak heights. Journal of Chromatography A 2005, 1092, 191-198.

116. Krieger, S. Comprehensive 2D-LC analysis of Chinese herbal medicine. $L C G C$ Europe 2014, 27, 677.

117. Horváth, K.; Fairchild, J.; Guiochon, G. Optimization strategies for off-line twodimensional liquid chromatography. Journal of Chromatography A 2009, 1216, 2511-2518.

118. Potts, L.; Stoll, D.; Li, X.; Carr, P. The impact of sampling time on peak capacity and analysis speed in on-line comprehensive two-dimensional liquid chromatography. Journal of Chromatography A 2010, 1217, 5700-5709.

119. Sarrut, M.; Szabolcs, F.; Janin-Bussat, M.-C.; Colas, O.; Guillarme, D.; Beck, A.; Heinisch, S. Analysis of antibody-drug conjugates by comprehensive on-line twodimensional hydrophobic interaction chromatography $\mathrm{x}$ reversed phase liquid chromatography hyphenated to high resolution mass spectrometry. II-Identification of sub-units for the characterization of even and odd load drug species. Journal of Chromatography B 2016, 1032, 91-102.

120. Sarrut, M.; Szabolcs, F.; Janin-Bussat, M.-C.; Colas, O.; Guillarme, D.; Beck, A.; Heinisch, S. Analysis of antibody-drug conjugates by comprehensive on-line twodimensional hydrophobic interaction chromatography $\mathrm{x}$ reversed phase liquid chromatography hyphenated to high resolution mass spectrometry. I - Optimization of separation conditions. Journal of Chromatography B 2016, 1032, 103-111. 
121. Vanhoenacker, G.; Vandenheede, I.; David, F.; Sandra, P.; Sandra, K.

Comprehensive two-dimensional liquid chromatography of therapeutic monoclonal antibody digests. Analytical and Bioanalytical Chemistry 2015, 407, 355-366.

122. Ikegami, T.; Hara, T.; Kimura, H.; Kobayashi, H.; Hosoya, K.; Cabrera, K.; Tanaka, N. Two-dimensional reversed-phase liquid chromatography using two monolithic silica $\mathrm{C} 18$ columns and diferent mobile phase modifiers in the two dimensions. Journal of Chromatography A 2006, 1106, 112-117.

123. Wang, J.; Xu, Y.; Wen, C.; Wang, Z. Application of a trap-free two-dimensional liquid chromatography combined with ion trap/time-of-flight mass spectrometry for separation and characterization of impurities and isomers in cefpiramide. Analytica Chimica Acta 2017, 992, 42-54.

124. Pellett, J.; Lukulay, P.; Mao, Y.; Bowen, W.; Reed, R.; Ma, M.; Munger, R. C.; Dolan, J. W.; Wrisley, L.; Medwid, K.; Toltl, N. P.; Chan, C. C.; Skibic, M.; Biswas, K.; Wells, K. A.; Snyder, L. R. "Orthogonal" separations for reversedphase liquid chromatography. Journal of Chromatography A 2006, 1101, 122-135.

125. Jandera, P.; Fischer, J.; Lahovská, H.; Novotná, K.; Cesla, P.; Kolárová, L. Twodimensional liquid chromatography normal-phase and reversed-phase separation of (co)oligomers. Journal of Chromatography A 2005, 1119, 3-10.

126. Gray, M. J.; Sweeney, A. P.; Dennis, G. R.; Slonecker, P. J.; Shalliker, R. A. Comprehensive coupled reversed-phase reversed-phase separations of a complex isomeric mixture. Analyst 2003, 128, 598-604.

127. Gray, M. J.; Dennis, G. R.; Slonecker, P. J.; Shalliker, R. A. Evaluation of the twodimensional reversed-phase-reversed-phase separations of low-molecular mass polystyrenes. Journal of Chromatography A 2003, 1015, 89-98.

128. Stoll, D. R.; Cohen, J. D.; Carr, P. W. Fast, comprehensive online two-dimensional high performance liquid chromatography through the use of high temperature ultrafast gradient elution reversed-phase liquid chromatography. Journal of Chromatography A 2006, 1122, 123-137.

129. Gray, M. J.; Dennis, G. R.; Slonecker, P. J.; Shalliker, R. A. Comprehensive twodimensional separations of complex mixtures using reversed-phase reversed-phase liquid chromatography. Journal of Chromatography A 2004, 1041, 101-110.

130. Gilar, M.; Olivova, P.; Daly, A. E.; Gebler, J. C. Two-dimensional separation of peptides using RP-RP-HPLC system with different $\mathrm{pH}$ in first and second separation dimensions. Journal of Separation Science 2005, 28, 1694-1703. 
131. Gilar, M.; Olivova, P.; Daly, A. E.; Gebler, J. C. Orthogonality of separation in two-dimensional liquid chromatography. Analytical Chemistry 2005, 77, 64266434.

132. Opiteck, G. J.; Jorgenson, J. W. Two-dimensional SEC/RPLC coupled to mass spectrometry for the analysis of peptides. Analytical Chemistry 1997, 69, 22832291.

133. D'Attoma, A.; Grivel, C.; Heinisch, S. On-line comprehensive two-dimensional separations of charged compounds using reversed-phase high performance liquid chromatography and hydrophilic interaction chromatography. Part I: Orthogonality and practical peak capacity considerations. Journal of Chromatography A 2012, 1262, 148-159.

134. François, I.; de Villiers, A.; Sandra, P. Considerations on the possibilities and limitations of comprehensive normal phase-reversed phase liquid chromatography (NPLC x RPLC). Journal of Separation Science 2006, 29, 492-498.

135. Blahová, E.; Jandera, P.; Cacciola, F.; Mondello, L. Two-dimensional and serial column reversed-phase separation of phenolic antioxidants on octadecyl-, polyethyleneglycol-, and pentafluorophenylpropyl-silica columns. Journal of Separation Science 2006, 29, 555-566.

136. Kalili, K. M.; de Villiers, A. Off-line comprehensive two-dimensional hydrophilic interaction $\mathrm{x}$ reversed phase liquid chromatographic analysis of green tea phenolics. Journal of Separation Science 2010, 33, 853-863.

137. François, I.; de Villiers, A.; Tienpont, B.; David, F.; Sandra, P. Comprehensive two-dimensional liquid chromatography applying two parallel columns in the second dimension. Journal of Chromatography A 2008, 1178, 33-42.

138. Dugo, P.; Kumm, T.; Crupi, M. L.; Cotroneo, A.; Mondello, L. Comprehensive two-dimensional liquid chromatography combined with mass spectrometric detection in the analyses of triacylglycerols in natural lipidic matrixes. Journal of Chromatography A 2006, 1112, 269-275.

139. Jones, D. R.; Boysen, G.; Miller, G. Novel multi-mode ultra performance liquid chromatography-tandem mass spectrometry assay for profiling enantiomeric hydroxywarfarins and warfarin in human plasma. Journal of Chromatography $B$ 2011, 879, 1056-1062.

140. Swortwood, M. J.; Boland, D. M.; DeCaprio, A. P. Determination of 32 cathinone derivatives and other designer drugs in serum by comprehensive LC-QQQ-MS/MS analysis. Analytical and Bioanalytical Chemistry 2013, 405, 1383-1397. 
141. Favretto, D.; Pascali, J. P.; Tagliaro, F. New challenges and innovation in forensic toxicology: Focus on the "New Psychoactive Substances". Journal of

Chromatography A 2013, 1287, 84-95.

142. Allen, K. R. Screening for drugs of abuse: which matrix, oral fluid or urine? Annals of Clinical Biochemistry 2011, 48, 531-541.

143. Wohlfarth, A.; Scheidweilder, K. B.; Chen, X.; Liu, H.; Huestis, M. A. Qualitative confirmation of 9 synthetic cannabinoids and 20 metabolites in human urine using LC-MS/MS and library search. Analytical Chemistry 2013, 85, 3730-3738.

144. Montesano, C.; Vannutelli, G.; Gregori, A.; Ripani, L.; Compagnone, D.; Curini, R.; Sergi, M. Broad screening and identification of novel psychoactive substances in plasma by high-performance liquid chromatography-high resolution mass spectrometry and post-run library matching. Journal of Analytical Toxicology 2016, $40,519-528$.

145. Seither, J. Z.; Arroyo-Mora, L. E.; Hindle, R.; DeCaprio, A. P. Development of a compound database and high resolution MS/MS spectral library for the identification of designer drugs by LC-QTOF-MS. Unpublished Work, 2014.

146. Gaudette, F.; Benito, J.; Steagall, P.; Beaudry, F. Assessment of tandem mass spectrometry and high-resolution mass spectrometry for the analysis of bupivacaine in plasma. Biomedical Chromatography 2015, 29, 1724-1730.

147. Seither, J. Z.; Hindle, R.; Arroyo-Mora, L. E.; DeCaprio, A. P. Systematic analysis of novel psychoactive substances. I. Development of a compound database and HRMS spectral library. Forensic Chemistry 2018, 9, 12-20.

148. Peters, F. T.; Drummer, O. H.; Musshoff, F. Validation of new methods. Forensic Science International 2007, 165, 216-224.

149. Scientific Working Group for Forensic Toxicology (SWGTOX) Standard Practices for Method Validation in Forensic Toxicology; 13.

150. Toxicology Subcommittee of the Organization of Scientific Area Committees Standard Practices for Method Validation in Forensic Toxicology; 17.

151. Shanks, K. G.; Dahn, T.; Terrell, A. R. Detection of JWH-018 and JWH-073 by UPLC-MS-MS in postmortem whole blood casework. Journal of Analytical Toxicology 2012, 31, 333-337.

152. Sasaki, C.; Saito, T.; Shinozuka, T.; Irie, W.; Murakami, C.; Maeda, K.; Nakamura, N.; Oishi, M.; Nakamura, S.; Kurihara, K. A case of death caused by abuse of a synthetic cannaibinoid $N$-1-naphthalenyl-1-pentyl- $1 H$-indole-3-carboxamide. Forensic Toxicology 2015, 33, 165-169. 
153. Hasegawa, K.; Wurita, A.; Suzuku, O. Postmortem distribution of ABCHMINACA, 5-fluoro-AMB, and diphenidine in body fluids and solid tissues in a fatal poisoning case: usefulness of adipose tissue for detection of the drugs in unchanged forms. Forensic Toxicology 2015, 33, 45-53.

154. Kronstrand, R.; Brinkhagen, L.; Birath-Karlsson, C.; Roman, M.; Josefsson, M. LCQTOF-MS as a superior strategy to immunoassay for the comprehensive analysis of synthetic cannabinoids in urine. Analytical and Bioanalytical Chemistry 2014, 406, 3599-3609.

155. Castaneto, M. S.; Wohlfarth, A.; Desrosiers, N. A.; Hartman, R. L.; Gorelick, D. A.; Huestis, M. A. Synthetic cannabinoids pharmacokinetics and detection methods in biological matrices. Drug Metabolism Reviews 2015, 47, 124-174.

156. Kneisel, S.; Auwärter, V. Analysis of 30 synthetic cannabinoids in serum by liquid chromatography-electrospray ionization tandem mass spectrometry after liquidliquid extraction. Journal of Mass Spectrometry 2012, 47, 825-835.

157. Kneisel, S.; Speck, M.; Moosmann, B.; Corneillie, T. M.; Butlin, N. G.; Auwärter, V. LC/ESI-MS/MS method for quantification of 28 synthetic cannabinoids in neat oral fluid and its application to preliminary studies on their detection windows. Analytical and Bioanalytical Chemistry 2013, 405, 4691-4706.

158. Logan, B. K.; Kacinko, S. L.; McMullin, M. M.; Xu, A. Technical Bulletin: NMS Labs test for JWH-018, JWH-019, JWH-073, JWH-250 and AM-2201 Primary Monohydroxy Metabolite in Human Urine; 11.

159. Kneisel, S.; Auwärter, V.; Kempf, J. Analysis of 30 synthetic cannabinoids in oral fluid using liquid chromatography-electrospray ionization tandem mass spectrometry. Drug Testing and Analysis 2012, 5, 657-669.

160. Scheidweilder, K. B.; Huestis, M. A. Simultaneous quantification of 20 synthetic cannabinoids and 21 metabolites, and semi-quantification of 12 alkyl hydroxy metabolites in human urine by liquid chromatography-tandem mass spectrometry. Journal of Chromatography A 2014, 1327, 105-117.

161. Salomone, A.; Gerace, E.; D'Urso, F.; Di Corcia, D.; Vincenti, M. Simultaneous analysis of several sythetic cannabinoids, THC, CBD and CBN, in hair by ultrahigh performance liquid chromatography tandem mass spectrometry. Method validation and application to real samples. Journal of Mass Spectrometry 2012, 47, 604-610.

162. Li, X.; Uboh, C. E.; Soma, L. R.; Liu, Y.; Guan, F.; Aurand, C. R.; Bell, D. S.; You, Y.; Chen, J.; Maylin, G. A. Sensitive hydrophilic interaction liquid chromatography/tandem mass spectrometry method for rapid detection, quantification and confirmation of cathinone-derived designer drugs for doping 
control in equine plasma. Rapid Communications in Mass Spectrometry 2014, 28 , 217-229.

163. Frank, M. Agilent 1290 Infinity 2D-LC Solution: A new flexible and user-friendly 2D-LC solution for the most complex samples. 2012.

164. Eiceman, G. A.; Karpas, Z.; Hill Jr., H. H. Introduction to Ion Mobility Spectrometry. In Ion Mobility Spectrometry, 3rd ed.; Taylor \& Francis: Boca Raton, 2014.

165. Eiceman, G. A.; Karpas, Z.; Hill Jr., H. H. Drugs of Abuse. In Ion Mobility Spectrometry, 3rd ed.; Taylor \& Francis: Boca Raton, 2014.

166. Manicke, N. E.; Belford, M. Separation of opiate isomers using electrospray ionization and paper spray coupled to high-field asymmetric waveform ion mobility spectrometry. Journal of the American Society for Mass Spectrometry 2015, 26, 701-705.

167. Bell, D. S.; Shollenberger, D.; Cramer, H. Evaluation of retention and selectivity using biphenyl stationary phases. LC-GC North America 2017, 35, 360-365.

168. Euerby, M. R.; Petersson, P.; Campbell, W.; Roe, W. Chromatographic classification and comparison of commercially available reversed-phase liquid chromatographic columns containing phenyl moieties using principal component analysis. Journal of Chromatography A 2007, 1154, 138-151.

169. Euerby, M. R.; Petersson, P. Chromatographic classification and comparison of commercially available reversed-phase liquid chromatographic columns containing polar embedded groups/amino endcappings using principal component analysis. Journal of Chromatography A 2004, 1088, 1-15.

170. Layne, J. Characterization and comparison of the chromatographic performance of conventional, polar-embedded, and polar-endcapped reversed-phase liquid chromatography stationary phases. Journal of Chromatography A 2002, 957, 149164.

171. Marchand, D. H.; Croes, K.; Dolan, J. W.; Snyder, L. R.; Henry, R. A.; Kallury, K. M. R.; Waite, S.; Carr, P. W. Column selectivity in reversed-phase liquid chromatography: VIII. Phenylalkyl and fluoro-substituted columns. Journal of Chromatography A 2005, 1062, 65-78.

\section{APPENDICES}




\begin{tabular}{|c|c|c|c|}
\hline Compound Name & Formula & $\begin{array}{l}\text { Accurate } \\
\text { Mass }\end{array}$ & FIU Number \\
\hline$( \pm)$-Ethylphenidate & $\mathrm{C} 15 \mathrm{H} 21 \mathrm{NO} 2$ & 247.1572 & FIU 0279 \\
\hline 2,4,6-Trimethoxyamphetamine & C12H19NO3 & 225.1365 & FIU 0280 \\
\hline 2-Bromoamphetamine & C9H12BrN & 213.0153 & FIU 0281 \\
\hline 2-Chloroamphetamine & $\mathrm{C} 9 \mathrm{H} 12 \mathrm{ClN}$ & 169.0658 & FIU 0282 \\
\hline 2-methoxy Ketamine & $\mathrm{C} 14 \mathrm{H} 19 \mathrm{NO} 2$ & 233.1416 & FIU 0283 \\
\hline 2-Methoxyamphetamine & $\mathrm{C} 10 \mathrm{H} 15 \mathrm{NO}$ & 165.1154 & FIU 0284 \\
\hline 3,4-EDMC & $\mathrm{C} 12 \mathrm{H} 15 \mathrm{NO} 3$ & 221.1052 & FIU 0285 \\
\hline 3-Bromoamphetamine & $\mathrm{C} 9 \mathrm{H} 12 \mathrm{BrN}$ & 213.0153 & FIU 0286 \\
\hline 3-Chloroamphetamine & $\mathrm{C} 9 \mathrm{H} 12 \mathrm{ClN}$ & 169.0658 & FIU 0287 \\
\hline 3-Iodoamphetamine & C9H12IN & 261.0014 & FIU 0288 \\
\hline 3-Methoxyamphetamine & $\mathrm{C} 10 \mathrm{H} 15 \mathrm{NO}$ & 165.1154 & FIU 0289 \\
\hline 4-Bromoamphetamine & $\mathrm{C} 9 \mathrm{H} 12 \mathrm{BrN}$ & 213.0153 & FIU 0290 \\
\hline 4-Chloroamphetamine & $\mathrm{C} 9 \mathrm{H} 12 \mathrm{ClN}$ & 169.0658 & FIU 0291 \\
\hline 4-Hydroxyamphetamine & $\mathrm{C} 9 \mathrm{H} 13 \mathrm{NO}$ & 151.0997 & FIU 0292 \\
\hline 4-Methoxyamphetamine & $\mathrm{C} 10 \mathrm{H} 15 \mathrm{NO}$ & 165.1154 & FIU 0293 \\
\hline 4-MTA & $\mathrm{C} 10 \mathrm{H} 15 \mathrm{NS}$ & 181.0925 & FIU 0294 \\
\hline $\begin{array}{l}\text { 4-MTA (hydrochloride } \\
\text { preparation) }\end{array}$ & C10H15NS & 181.0925 & FIU 0295 \\
\hline D-Amphetamine & $\mathrm{C} 9 \mathrm{H} 13 \mathrm{~N}$ & 135.1048 & FIU 0296 \\
\hline deschloro-N-ethyl-Ketamine & C14H19NO & 217.1467 & FIU 0297 \\
\hline Diclofensine & $\mathrm{C} 17 \mathrm{H} 17 \mathrm{Cl} 2 \mathrm{NO}$ & 321.0687 & FIU 0298 \\
\hline DOI & C11H16INO2 & 321.0226 & FIU 0299 \\
\hline HMA & $\mathrm{C} 10 \mathrm{H} 15 \mathrm{NO} 2$ & 181.1103 & FIU 0300 \\
\hline Lisdexamfetamine & $\mathrm{C} 15 \mathrm{H} 25 \mathrm{~N} 3 \mathrm{O}$ & 263.1998 & FIU 0301 \\
\hline Propylhexedrine & $\mathrm{C} 10 \mathrm{H} 21 \mathrm{~N}$ & 155.1674 & FIU 0302 \\
\hline CMP & $\mathrm{C} 10 \mathrm{H} 17 \mathrm{~N}$ & 151.1361 & FIU 0303 \\
\hline 2,5-DMMA & $\mathrm{C} 12 \mathrm{H} 19 \mathrm{NO} 2$ & 209.1416 & FIU 0304 \\
\hline 3,4-DHMA & $\mathrm{C} 10 \mathrm{H} 15 \mathrm{NO} 2$ & 181.1103 & FIU 0305 \\
\hline 4-bromo-2,5-DMMA & $\mathrm{C} 12 \mathrm{H} 18 \mathrm{BrNO} 2$ & 287.0521 & FIU 0306 \\
\hline $\begin{array}{c}\text { para- } \\
\text { Methoxymethamphetamine }\end{array}$ & $\mathrm{C} 11 \mathrm{H} 17 \mathrm{NO}$ & 179.1310 & FIU 0307 \\
\hline Methiopropamine & C8H13NS & 155.0769 & FIU 0308 \\
\hline N,N-DMA & $\mathrm{C} 11 \mathrm{H} 17 \mathrm{~N}$ & 163.1361 & FIU 0309 \\
\hline $2 \mathrm{C}-\mathrm{G}$ & $\mathrm{C} 12 \mathrm{H} 19 \mathrm{NO} 2$ & 209.1416 & FIU 0310 \\
\hline $2 \mathrm{C}-\mathrm{T}$ & $\mathrm{C} 11 \mathrm{H} 17 \mathrm{NO} 2 \mathrm{~S}$ & 227.0980 & FIU 0311 \\
\hline $2 \mathrm{C}-\mathrm{T}-7$ & $\mathrm{C} 13 \mathrm{H} 21 \mathrm{NO} 2 \mathrm{~S}$ & 255.1293 & FIU 0312 \\
\hline 2C-TFM & $\mathrm{C} 11 \mathrm{H} 14 \mathrm{~F} 3 \mathrm{NO} 2$ & 249.0977 & FIU 0313 \\
\hline bk-2C-B & $\mathrm{C} 10 \mathrm{H} 12 \mathrm{BrNO} 3$ & 273.0001 & FIU 0314 \\
\hline
\end{tabular}




\begin{tabular}{|c|c|c|c|}
\hline 3C-B-fly & $\mathrm{C} 13 \mathrm{H} 16 \mathrm{BrNO} 2$ & 297.0364 & FIU 0315 \\
\hline $3 \mathrm{C}-\mathrm{P}$ & $\mathrm{C} 14 \mathrm{H} 23 \mathrm{NO} 3$ & 253.1678 & FIU 0316 \\
\hline N-methyl-2-AI & $\mathrm{C} 10 \mathrm{H} 13 \mathrm{~N}$ & 147.1048 & FIU 0317 \\
\hline Escaline & $\mathrm{C} 12 \mathrm{H} 19 \mathrm{NO} 3$ & 225.1365 & FIU 0318 \\
\hline Mescaline & C11H17NO3 & 211.1208 & FIU 0319 \\
\hline 2-Amino-1-phenylbutane & $\mathrm{C} 10 \mathrm{H} 15 \mathrm{~N}$ & 149.1204 & FIU 0320 \\
\hline 2-Ethylamino-1-phenylbutane & $\mathrm{C} 12 \mathrm{H} 19 \mathrm{~N}$ & 177.1517 & FIU 0321 \\
\hline 4-CAB & $\mathrm{C} 10 \mathrm{H} 14 \mathrm{ClN}$ & 183.0815 & FIU 0322 \\
\hline Cathine & $\mathrm{C} 9 \mathrm{H} 13 \mathrm{NO}$ & 151.0997 & FIU 0323 \\
\hline (R)-(-)-MT-45 & $\mathrm{C} 24 \mathrm{H} 32 \mathrm{~N} 2$ & 348.2565 & FIU 0324 \\
\hline$(\mathrm{S})-(+)-\mathrm{MT}-45$ & $\mathrm{C} 24 \mathrm{H} 32 \mathrm{~N} 2$ & 348.2565 & FIU 0325 \\
\hline 2,3-Dichlorophenylpiperazine & $\mathrm{C} 10 \mathrm{H} 12 \mathrm{Cl} 2 \mathrm{~N} 2$ & 230.0378 & FIU 0326 \\
\hline MBZP & C12H18N2 & 190.1470 & FIU 0327 \\
\hline MT-45 & $\mathrm{C} 24 \mathrm{H} 32 \mathrm{~N} 2$ & 348.2565 & FIU 0328 \\
\hline Mepirapim & $\mathrm{C} 19 \mathrm{H} 27 \mathrm{~N} 3 \mathrm{O}$ & 313.2154 & FIU 0329 \\
\hline 2-Fluoroisocathinone & C9H10FNO & 167.0746 & FIU 0330 \\
\hline 3,4-Dimethylethcathinone & $\mathrm{C} 13 \mathrm{H} 19 \mathrm{NO}$ & 205.1467 & FIU 0331 \\
\hline $\begin{array}{l}\text { 4-methoxy-N,N- } \\
\text { Dimethylcathinone }\end{array}$ & $\mathrm{C} 12 \mathrm{H} 17 \mathrm{NO} 2$ & 207.1259 & FIU 0332 \\
\hline $\begin{array}{l}\text { 4-Methylethcathinone } \\
\text { metabolite }(( \pm) \text {-Ephedrine } \\
\text { stereochemistry) }\end{array}$ & $\mathrm{C} 12 \mathrm{H} 19 \mathrm{NO}$ & 193.1467 & FIU 0333 \\
\hline $\begin{array}{l}\text { 4-Methylethcathinone } \\
\text { metabolite }(( \pm)- \\
\text { Pseudoephedrine } \\
\text { stereochemistry) }\end{array}$ & $\mathrm{C} 12 \mathrm{H} 19 \mathrm{NO}$ & 193.1467 & FIU 0334 \\
\hline N-ethyl-N-Methylcathinone & $\mathrm{C} 12 \mathrm{H} 17 \mathrm{NO}$ & 191.1310 & FIU 0335 \\
\hline Isopentedrone & $\mathrm{C} 12 \mathrm{H} 17 \mathrm{NO}$ & 191.1310 & FIU 0336 \\
\hline Mephedrone & $\mathrm{C} 11 \mathrm{H} 15 \mathrm{NO}$ & 177.1154 & FIU 0337 \\
\hline $\begin{array}{l}\text { Mephedrone metabolite }(( \pm)- \\
\text { Ephedrine stereochemistry) }\end{array}$ & $\mathrm{C} 11 \mathrm{H} 17 \mathrm{NO}$ & 179.1310 & FIU 0338 \\
\hline $\begin{array}{c}\text { Mephedrone metabolite }(( \pm)- \\
\text { Pseudoephedrine } \\
\text { stereochemistry })\end{array}$ & $\mathrm{C} 11 \mathrm{H} 17 \mathrm{NO}$ & 179.1310 & FIU 0339 \\
\hline NRG-3 & $\mathrm{C} 16 \mathrm{H} 19 \mathrm{NO}$ & 241.1467 & FIU 0340 \\
\hline $\begin{array}{l}\text { Pentedrone metabolite }(( \pm)- \\
\text { Ephedrine stereochemistry) }\end{array}$ & C12H19NO & 193.1467 & FIU 0341 \\
\hline $\begin{array}{c}\text { Pentedrone metabolite }(( \pm)- \\
\text { Pseudoephedrine } \\
\text { stereochemistry) } \\
\end{array}$ & C12H19NO & 193.1467 & FIU 0342 \\
\hline Benzedrone & C17H19NO & 253.1467 & FIU 0343 \\
\hline (-)-(S)-Cathinone & $\mathrm{C} 9 \mathrm{H} 11 \mathrm{NO}$ & 149.0841 & FIU 0344 \\
\hline
\end{tabular}




\begin{tabular}{|c|c|c|c|}
\hline 2,3-Dimethylethcathinone & C13H19NO & 205.1467 & FIU 0345 \\
\hline 2,4-Dimethylethcathinone & C13H19NO & 205.1467 & FIU 0346 \\
\hline Diethylcathinone & $\mathrm{C} 13 \mathrm{H} 19 \mathrm{NO}$ & 205.1467 & FIU 0347 \\
\hline 2,3-Dimethylmethcathinone & $\mathrm{C} 12 \mathrm{H} 17 \mathrm{NO}$ & 191.1310 & FIU 0348 \\
\hline 2,4-Dimethylmethcathinone & $\mathrm{C} 12 \mathrm{H} 17 \mathrm{NO}$ & 191.1310 & FIU 0349 \\
\hline $\begin{array}{l}\text { 3,4-Dimethylmethcathinone } \\
\text { metabolite }(( \pm) \text {-Ephedrine } \\
\text { stereochemistry })\end{array}$ & $\mathrm{C} 12 \mathrm{H} 19 \mathrm{NO}$ & 193.1467 & FIU 0350 \\
\hline $\begin{array}{l}\text { 3,4-Dimethylmethcathinone } \\
\text { metabolite }(( \pm)- \\
\text { Pseudoephedrine } \\
\text { stereochemistry })\end{array}$ & C12H19NO & 193.1467 & FIU 0351 \\
\hline 3-Bromomethcathinone & $\mathrm{C} 10 \mathrm{H} 12 \mathrm{BrNO}$ & 241.0102 & FIU 0352 \\
\hline 4-Bromomethcathinone & $\mathrm{C} 10 \mathrm{H} 12 \mathrm{BrNO}$ & 241.0102 & FIU 0353 \\
\hline $\begin{array}{l}\text { 4-Fluoromethcathinone } \\
\text { metabolite }(( \pm) \text {-Ephedrine } \\
\text { stereochemistry) }\end{array}$ & C10H14FNO & 183.1059 & FIU 0354 \\
\hline $\begin{array}{l}\text { 4-Fluoromethcathinone } \\
\text { metabolite }(( \pm)- \\
\text { Pseudoephedrine } \\
\text { stereochemistry }) \\
\end{array}$ & C10H14FNO & 183.1059 & FIU 0355 \\
\hline $\begin{array}{c}(-)-3,4-\text { Methylenedioxy } \\
\text { Pyrovalerone }\end{array}$ & $\mathrm{C} 16 \mathrm{H} 21 \mathrm{NO} 3$ & 275.1521 & FIU 0356 \\
\hline $\begin{array}{c}\text { (+)-3,4-Methylenedioxy } \\
\text { Pyrovalerone }\end{array}$ & $\mathrm{C} 16 \mathrm{H} 21 \mathrm{NO} 3$ & 275.1521 & FIU 0357 \\
\hline 2,3-MDA & $\mathrm{C} 10 \mathrm{H} 13 \mathrm{NO} 2$ & 179.0946 & FIU 0358 \\
\hline 2,3-MDMA & $\mathrm{C} 11 \mathrm{H} 15 \mathrm{NO} 2$ & 193.1103 & FIU 0359 \\
\hline 3,4-MDMA & $\mathrm{C} 11 \mathrm{H} 15 \mathrm{NO} 2$ & 193.1103 & FIU 0360 \\
\hline $\begin{array}{l}\text { 3,4-Methylenedioxy-5- } \\
\text { methylethcathinone }\end{array}$ & C13H17NO3 & 235.1208 & FIU 0361 \\
\hline $\begin{array}{l}\text { 3,4-Methylenedioxy-N- } \\
\text { benzylcathinone }\end{array}$ & $\mathrm{C} 17 \mathrm{H} 17 \mathrm{NO} 3$ & 283.1208 & FIU 0362 \\
\hline Methylenedioxy Pyrovalerone & $\mathrm{C} 16 \mathrm{H} 21 \mathrm{NO} 3$ & 275.1521 & FIU 0363 \\
\hline $\begin{array}{l}\text { Methylenedioxy Pyrovalerone } \\
\text { Metabolite } 1\end{array}$ & $\mathrm{C} 16 \mathrm{H} 23 \mathrm{NO} 3$ & 277.1678 & FIU 0364 \\
\hline $\begin{array}{l}\text { Methylenedioxy Pyrovalerone } \\
\text { metabolite } 2\end{array}$ & $\mathrm{C} 15 \mathrm{H} 21 \mathrm{NO} 3$ & 263.1521 & FIU 0365 \\
\hline $\begin{array}{c}\text { N-acetyl-3,4- } \\
\text { Methylenedioxymethcathinone }\end{array}$ & C13H15NO4 & 249.1001 & FIU 0366 \\
\hline N-hydroxy MDA & C10H13NO3 & 195.0895 & FIU 0367 \\
\hline Myristicin & $\mathrm{C} 11 \mathrm{H} 12 \mathrm{O} 3$ & 192.0786 & FIU 0368 \\
\hline Piperonyl methyl ketone & $\mathrm{C} 10 \mathrm{H} 10 \mathrm{O} 3$ & 178.0630 & FIU 0369 \\
\hline Safrole & $\mathrm{C} 10 \mathrm{H} 10 \mathrm{O} 2$ & 162.0681 & FIU 0370 \\
\hline
\end{tabular}




\begin{tabular}{|c|c|c|c|}
\hline 25B-NBOMe & $\mathrm{C} 18 \mathrm{H} 22 \mathrm{BrNO} 3$ & 379.0783 & FIU 0371 \\
\hline 25C-NBOMe & $\mathrm{C} 18 \mathrm{H} 22 \mathrm{ClNO} 3$ & 335.1288 & FIU 0372 \\
\hline 25D-NBOMe & $\mathrm{C} 19 \mathrm{H} 25 \mathrm{NO} 3$ & 315.1834 & FIU 0373 \\
\hline 25E-NBOMe & $\mathrm{C} 20 \mathrm{H} 27 \mathrm{NO} 3$ & 329.1991 & FIU 0374 \\
\hline 25G-NBOMe & $\mathrm{C} 20 \mathrm{H} 27 \mathrm{NO} 3$ & 329.1991 & FIU 0375 \\
\hline 25H-NBOMe & $\mathrm{C} 18 \mathrm{H} 23 \mathrm{NO} 3$ & 301.1678 & FIU 0376 \\
\hline 25H-NBOMe imine analog & $\mathrm{C} 18 \mathrm{H} 21 \mathrm{NO} 3$ & 299.1521 & FIU 0377 \\
\hline $25 \mathrm{I}-\mathrm{NBF}$ & C17H19FINO2 & 415.0445 & FIU 0378 \\
\hline 25I-NBMD & C18H20INO4 & 441.0437 & FIU 0379 \\
\hline 25I-NBOMe 3-methoxy isomer & C18H22INO3 & 427.0644 & FIU 0380 \\
\hline 25I-NBOMe 4-methoxy isomer & C18H22INO3 & 427.0644 & FIU 0381 \\
\hline 25I-NBOMe imine analog & C18H20INO3 & 425.0488 & FIU 0382 \\
\hline $25 \mathrm{~N}-\mathrm{NBOMe}$ & $\mathrm{C} 18 \mathrm{H} 22 \mathrm{~N} 2 \mathrm{O} 5$ & 346.1529 & FIU 0383 \\
\hline 25T2-NBOMe & $\mathrm{C} 19 \mathrm{H} 25 \mathrm{NO} 3 \mathrm{~S}$ & 347.1555 & FIU 0384 \\
\hline 30C-NBOMe & $\mathrm{C} 20 \mathrm{H} 26 \mathrm{ClNO} 5$ & 395.1500 & FIU 0385 \\
\hline 3-methoxy PCP & $\mathrm{C} 18 \mathrm{H} 27 \mathrm{NO}$ & 273.2093 & FIU 0386 \\
\hline 4-methoxy PCP & $\mathrm{C} 18 \mathrm{H} 27 \mathrm{NO}$ & 273.2093 & FIU 0387 \\
\hline PCEEA & $\mathrm{C} 16 \mathrm{H} 25 \mathrm{NO}$ & 247.1936 & FIU 0388 \\
\hline PCMPA & $\mathrm{C} 16 \mathrm{H} 25 \mathrm{NO}$ & 247.1936 & FIU 0389 \\
\hline $\mathrm{PCPr}$ & $\mathrm{C} 15 \mathrm{H} 23 \mathrm{~N}$ & 217.1830 & FIU 0390 \\
\hline Benocyclidine & $\mathrm{C} 19 \mathrm{H} 25 \mathrm{NS}$ & 299.1708 & FIU 0391 \\
\hline $\begin{array}{l}\text { 2-methyl- } \alpha \text { - } \\
\text { Pyrrolidinopropiophenone }\end{array}$ & $\mathrm{C} 14 \mathrm{H} 19 \mathrm{NO}$ & 217.1467 & FIU 0392 \\
\hline $\begin{array}{c}\text { 3,4-dimethoxy- } \alpha- \\
\text { Pyrrolidinopentiophenone }\end{array}$ & $\mathrm{C} 17 \mathrm{H} 25 \mathrm{NO} 3$ & 291.1834 & FIU 0393 \\
\hline $\begin{array}{c}\text { 3'-fluoro- } \alpha \text { - } \\
\text { Pyrrolidinopropiophenone }\end{array}$ & $\mathrm{C} 13 \mathrm{H} 16 \mathrm{FNO}$ & 221.1216 & FIU 0394 \\
\hline $\begin{array}{c}\text { 3-methyl- } \alpha- \\
\text { Pyrrolidinopropiophenone }\end{array}$ & C14H19NO & 217.1467 & FIU 0395 \\
\hline $\begin{array}{c}\text { 4'-Methyl-N- } \\
\text { methylhexanophenone }\end{array}$ & $\mathrm{C} 14 \mathrm{H} 21 \mathrm{NO}$ & 219.1623 & FIU 0396 \\
\hline $\begin{array}{c}\text { 4-fluoro- } \alpha- \\
\text { Pyrrolidinobutiophenone }\end{array}$ & $\mathrm{C} 14 \mathrm{H} 18 \mathrm{FNO}$ & 235.1372 & FIU 0397 \\
\hline $\begin{array}{c}\text { 4-fluoro- } \alpha- \\
\text { Pyrrolidinopentiophenone }\end{array}$ & $\mathrm{C} 15 \mathrm{H} 20 \mathrm{FNO}$ & 249.1529 & FIU 0398 \\
\hline $\begin{array}{c}\text { 4'-fluoro- } \alpha \text { - } \\
\text { Pyrrolidinopropiophenone }\end{array}$ & C13H16FNO & 221.1216 & FIU 0399 \\
\hline $\begin{array}{l}\text { 4-methoxy- } \alpha \text { - } \\
\text { Pyrrolidinobutiophenone }\end{array}$ & $\mathrm{C} 15 \mathrm{H} 21 \mathrm{NO} 2$ & 247.1572 & FIU 0400 \\
\hline $\begin{array}{c}\text { 4-methoxy- } \alpha- \\
\text { Pyrrolidinopentiophenone }\end{array}$ & $\mathrm{C} 16 \mathrm{H} 23 \mathrm{NO} 2$ & 261.1729 & FIU 0401 \\
\hline
\end{tabular}




\begin{tabular}{|c|c|c|c|}
\hline $\begin{array}{l}\text { 4-Methyl- } \alpha \text { - } \\
\text { ethylaminobutiophenone }\end{array}$ & $\mathrm{C} 13 \mathrm{H} 19 \mathrm{NO}$ & 205.1467 & FIU 0402 \\
\hline $\begin{array}{c}\text { 4-Methyl- } \alpha- \\
\text { ethylaminopentiophenone }\end{array}$ & $\mathrm{C} 14 \mathrm{H} 21 \mathrm{NO}$ & 219.1623 & FIU 0403 \\
\hline$\alpha$-Ethylaminopentiophenone & $\mathrm{C} 13 \mathrm{H} 19 \mathrm{NO}$ & 205.1467 & FIU 0404 \\
\hline$\alpha$-Pyrrolidinobutiophenone & $\mathrm{C} 14 \mathrm{H} 19 \mathrm{NO}$ & 217.1467 & FIU 0405 \\
\hline$\alpha$-Pyrrolidinobutiothiophenone & $\mathrm{C} 12 \mathrm{H} 17 \mathrm{NOS}$ & 223.1031 & FIU 0406 \\
\hline $\begin{array}{l}\alpha \text {-Pyrrolidinopentiophenone } \\
\text { metabolite } 1\end{array}$ & $\mathrm{C} 15 \mathrm{H} 23 \mathrm{NO}$ & 233.1780 & FIU 0407 \\
\hline $\begin{array}{c}\alpha- \\
\text { Pyrrolidinopentiothiophenone }\end{array}$ & C13H19NOS & 237.1187 & FIU 0408 \\
\hline 4-fluoro PV8 & C17H24FNO & 277.1842 & FIU 0409 \\
\hline 4-fluoro PV9 & $\mathrm{C} 18 \mathrm{H} 26 \mathrm{FNO}$ & 291.1998 & FIU 0410 \\
\hline 4-methoxy PV8 & $\mathrm{C} 18 \mathrm{H} 27 \mathrm{NO} 2$ & 289.2042 & FIU 0411 \\
\hline 4-methoxy PV9 & $\mathrm{C} 19 \mathrm{H} 29 \mathrm{NO} 2$ & 303.2198 & FIU 0412 \\
\hline PV8 & $\mathrm{C} 17 \mathrm{H} 25 \mathrm{NO}$ & 259.1936 & FIU 0413 \\
\hline PV9 & $\mathrm{C} 18 \mathrm{H} 27 \mathrm{NO}$ & 273.2093 & FIU 0414 \\
\hline Pyrovalerone & $\mathrm{C} 16 \mathrm{H} 23 \mathrm{NO}$ & 245.1780 & FIU 0415 \\
\hline 4-APB & $\mathrm{C} 11 \mathrm{H} 13 \mathrm{NO}$ & 175.0997 & FIU 0416 \\
\hline 4-APDB & $\mathrm{C} 11 \mathrm{H} 15 \mathrm{NO}$ & 177.1154 & FIU 0417 \\
\hline 5-APDB & $\mathrm{C} 11 \mathrm{H} 15 \mathrm{NO}$ & 177.1154 & FIU 0418 \\
\hline 5-EAPB & $\mathrm{C} 13 \mathrm{H} 17 \mathrm{NO}$ & 203.1310 & FIU 0419 \\
\hline 5-MAPB & $\mathrm{C} 12 \mathrm{H} 15 \mathrm{NO}$ & 189.1154 & FIU 0420 \\
\hline 5-MAPDB & $\mathrm{C} 12 \mathrm{H} 17 \mathrm{NO}$ & 191.1310 & FIU 0421 \\
\hline 6-APB & $\mathrm{C} 11 \mathrm{H} 13 \mathrm{NO}$ & 175.0997 & FIU 0422 \\
\hline 6-APDB & $\mathrm{C} 11 \mathrm{H} 15 \mathrm{NO}$ & 177.1154 & FIU 0423 \\
\hline 7-APB & $\mathrm{C} 11 \mathrm{H} 13 \mathrm{NO}$ & 175.0997 & FIU 0424 \\
\hline 3-Methylbuphedrone & $\mathrm{C} 12 \mathrm{H} 17 \mathrm{NO}$ & 191.1310 & FIU 0425 \\
\hline 4-Fluorobuphedrone & C11H14FNO & 195.1059 & FIU 0426 \\
\hline 4-Methylbuphedrone & $\mathrm{C} 12 \mathrm{H} 17 \mathrm{NO}$ & 191.1310 & FIU 0427 \\
\hline 4-methyl-N-Methylbuphedrone & $\mathrm{C} 13 \mathrm{H} 19 \mathrm{NO}$ & 205.1467 & FIU 0428 \\
\hline $\begin{array}{l}\text { Buphedrone metabolite }(( \pm)- \\
\text { Ephedrine stereochemistry })\end{array}$ & $\mathrm{C} 11 \mathrm{H} 17 \mathrm{NO}$ & 179.1310 & FIU 0429 \\
\hline $\begin{array}{l}\text { Buphedrone metabolite }(( \pm)- \\
\text { Pseudoephedrine } \\
\text { stereochemistry })\end{array}$ & $\mathrm{C} 11 \mathrm{H} 17 \mathrm{NO}$ & 179.1310 & FIU 0430 \\
\hline N-Ethylbuphedrone & $\mathrm{C} 12 \mathrm{H} 17 \mathrm{NO}$ & 191.1310 & FIU 0431 \\
\hline Dimethocaine & $\mathrm{C} 16 \mathrm{H} 26 \mathrm{~N} 2 \mathrm{O} 2$ & 278.1994 & FIU 0432 \\
\hline Nitracaine & $\mathrm{C} 16 \mathrm{H} 24 \mathrm{~N} 2 \mathrm{O} 4$ & 308.1736 & FIU 0433 \\
\hline (-)-11-nor-9-carboxy- $\Delta$ 9-THC & $\mathrm{C} 21 \mathrm{H} 28 \mathrm{O} 4$ & 344.1988 & FIU 0434 \\
\hline$( \pm$-Cannabichromene & $\mathrm{C} 21 \mathrm{H} 30 \mathrm{O} 2$ & 314.2246 & FIU 0435 \\
\hline
\end{tabular}




\begin{tabular}{|c|c|c|c|}
\hline$( \pm)-\mathrm{ORG} 28611$ & $\mathrm{C} 23 \mathrm{H} 33 \mathrm{~N} 3 \mathrm{O} 2$ & 383.2573 & FIU 0436 \\
\hline 5-fluoro NNEI & $\mathrm{C} 24 \mathrm{H} 23 \mathrm{FN} 2 \mathrm{O}$ & 374.1794 & FIU 0437 \\
\hline $\begin{array}{l}\text { 5-fluoro NNEI 2'-naphthyl } \\
\text { isomer }\end{array}$ & $\mathrm{C} 24 \mathrm{H} 23 \mathrm{FN} 2 \mathrm{O}$ & 374.1794 & FIU 0438 \\
\hline 5-fluoro SDB-005 & $\mathrm{C} 23 \mathrm{H} 21 \mathrm{FN} 2 \mathrm{O} 2$ & 376.1587 & FIU 0439 \\
\hline 5-fluoro SDB-006 & $\mathrm{C} 21 \mathrm{H} 23 \mathrm{FN} 2 \mathrm{O}$ & 338.1794 & FIU 0440 \\
\hline A-796260 & $\mathrm{C} 22 \mathrm{H} 30 \mathrm{~N} 2 \mathrm{O} 2$ & 354.2307 & FIU 0441 \\
\hline A-836339 & $\mathrm{C} 16 \mathrm{H} 26 \mathrm{~N} 2 \mathrm{O} 2 \mathrm{~S}$ & 310.1715 & FIU 0442 \\
\hline JW 618 & $\mathrm{C} 17 \mathrm{H} 14 \mathrm{~F} 6 \mathrm{~N} 2 \mathrm{O} 2$ & 392.0959 & FIU 0443 \\
\hline JW 642 & $\mathrm{C} 21 \mathrm{H} 20 \mathrm{~F} 6 \mathrm{~N} 2 \mathrm{O} 3$ & 462.1378 & FIU 0444 \\
\hline MN-25 & $\mathrm{C} 26 \mathrm{H} 37 \mathrm{~N} 3 \mathrm{O} 3$ & 439.2835 & FIU 0445 \\
\hline MN-25-2-methyl derivative & $\mathrm{C} 27 \mathrm{H} 39 \mathrm{~N} 3 \mathrm{O} 3$ & 453.2991 & FIU 0446 \\
\hline NNEI & $\mathrm{C} 24 \mathrm{H} 24 \mathrm{~N} 2 \mathrm{O}$ & 356.1889 & FIU 0447 \\
\hline NNEI 2'-naphthyl isomer & $\mathrm{C} 24 \mathrm{H} 24 \mathrm{~N} 2 \mathrm{O}$ & 356.1889 & FIU 0448 \\
\hline Salvinorin A & $\mathrm{C} 23 \mathrm{H} 28 \mathrm{O} 8$ & 432.1784 & FIU 0449 \\
\hline Salvinorin B & $\mathrm{C} 21 \mathrm{H} 26 \mathrm{O} 7$ & 390.1679 & FIU 0450 \\
\hline SDB-005 & $\mathrm{C} 23 \mathrm{H} 22 \mathrm{~N} 2 \mathrm{O} 2$ & 358.1681 & FIU 0451 \\
\hline SDB-006 & $\mathrm{C} 21 \mathrm{H} 24 \mathrm{~N} 2 \mathrm{O}$ & 320.1889 & FIU 0452 \\
\hline THCA-A & $\mathrm{C} 22 \mathrm{H} 30 \mathrm{O} 4$ & 358.2144 & FIU 0453 \\
\hline$\Delta 8$-THC & $\mathrm{C} 21 \mathrm{H} 30 \mathrm{O} 2$ & 314.2246 & FIU 0454 \\
\hline$\Delta 9-\mathrm{THC}$ & $\mathrm{C} 21 \mathrm{H} 30 \mathrm{O} 2$ & 314.2246 & FIU 0455 \\
\hline AM1248 azepane isomer & $\mathrm{C} 26 \mathrm{H} 34 \mathrm{~N} 2 \mathrm{O}$ & 390.2671 & FIU 0456 \\
\hline AM2201 benzimidazole analog & $\mathrm{C} 23 \mathrm{H} 21 \mathrm{FN} 2 \mathrm{O}$ & 360.1638 & FIU 0457 \\
\hline JZL 184 & $\mathrm{C} 27 \mathrm{H} 24 \mathrm{~N} 2 \mathrm{O} 9$ & 520.1482 & FIU 0458 \\
\hline KM 233 & $\mathrm{C} 25 \mathrm{H} 30 \mathrm{O} 2$ & 362.2246 & FIU 0459 \\
\hline KML29 & $\mathrm{C} 24 \mathrm{H} 21 \mathrm{~F} 6 \mathrm{NO} 7$ & 549.1222 & FIU 0460 \\
\hline LY2183240 & $\mathrm{C} 17 \mathrm{H} 17 \mathrm{~N} 5 \mathrm{O}$ & 307.1433 & FIU 0461 \\
\hline LY2183240 2'-isomer & C17H17N5O & 307.1433 & FIU 0462 \\
\hline SER-601 & $\mathrm{C} 28 \mathrm{H} 38 \mathrm{~N} 2 \mathrm{O} 2$ & 434.2933 & FIU 0463 \\
\hline Tetrahydrocannabivarin & $\mathrm{C} 19 \mathrm{H} 26 \mathrm{O} 2$ & 286.1933 & FIU 0464 \\
\hline Yangonin & C15H14O4 & 258.0892 & FIU 0465 \\
\hline Cannabidiolic Acid & $\mathrm{C} 22 \mathrm{H} 30 \mathrm{O} 4$ & 358.2144 & FIU 0466 \\
\hline Cannabigerol & $\mathrm{C} 21 \mathrm{H} 32 \mathrm{O} 2$ & 316.2402 & FIU 0467 \\
\hline EG-018 & $\mathrm{C} 28 \mathrm{H} 25 \mathrm{NO}$ & 391.1936 & FIU 0468 \\
\hline $\begin{array}{c}( \pm)-J W H 018 \mathrm{~N}-(2- \\
\text { hydroxypentyl) metabolite }\end{array}$ & $\mathrm{C} 24 \mathrm{H} 23 \mathrm{NO} 2$ & 357.1729 & FIU 0469 \\
\hline $\begin{array}{c}\begin{array}{c}( \pm)-J W H \\
\text { hydroxypentyl) metabolite }\end{array} \\
\end{array}$ & $\mathrm{C} 24 \mathrm{H} 23 \mathrm{NO} 2$ & 357.1729 & FIU 0470 \\
\hline $\begin{array}{c}\text { ( } \pm \text { )-JWH } 018 \mathrm{~N}-(4- \\
\text { hydroxypentyl) metabolite }\end{array}$ & $\mathrm{C} 24 \mathrm{H} 23 \mathrm{NO} 2$ & 357.1729 & FIU 0471 \\
\hline
\end{tabular}




\begin{tabular}{|c|c|c|c|}
\hline $\begin{array}{l}(\mathrm{R})-(-)-J W H 018 \mathrm{~N}-(4- \\
\text { hydroxypentyl) metabolite }\end{array}$ & $\mathrm{C} 24 \mathrm{H} 23 \mathrm{NO} 2$ & 357.1729 & FIU 0472 \\
\hline $\begin{array}{c}(\mathrm{S})-(+)-J W H 018 \mathrm{~N}-(4- \\
\text { hydroxypentyl) metabolite }\end{array}$ & $\mathrm{C} 24 \mathrm{H} 23 \mathrm{NO} 2$ & 357.1729 & FIU 0473 \\
\hline $\begin{array}{c}\text { 5-fluoro JWH } 018 \text { adamantyl } \\
\text { analog }\end{array}$ & C24H30FNO & 367.2311 & FIU 0474 \\
\hline $\begin{array}{l}\text { JWH } 018 \text { 2-hydroxyindole } \\
\text { metabolite }\end{array}$ & $\mathrm{C} 24 \mathrm{H} 23 \mathrm{NO} 2$ & 357.1729 & FIU 0475 \\
\hline $\begin{array}{c}\text { JWH } 018 \text { 4-hydroxyindole } \\
\text { metabolite }\end{array}$ & $\mathrm{C} 24 \mathrm{H} 23 \mathrm{NO} 2$ & 357.1729 & FIU 0476 \\
\hline $\begin{array}{c}\text { JWH } 018 \text { 5-hydroxyindole } \\
\text { metabolite }\end{array}$ & $\mathrm{C} 24 \mathrm{H} 23 \mathrm{NO} 2$ & 357.1729 & FIU 0477 \\
\hline $\begin{array}{c}\text { JWH } 018 \text { 6-hydroxyindole } \\
\text { metabolite }\end{array}$ & $\mathrm{C} 24 \mathrm{H} 23 \mathrm{NO} 2$ & 357.1729 & FIU 0478 \\
\hline $\begin{array}{c}\text { JWH } 018 \text { 7-hydroxyindole } \\
\text { metabolite }\end{array}$ & $\mathrm{C} 24 \mathrm{H} 23 \mathrm{NO} 2$ & 357.1729 & FIU 0479 \\
\hline $\begin{array}{c}\text { JWH } 018 \text { 8-quinolinyl } \\
\text { carboxamide }\end{array}$ & $\mathrm{C} 23 \mathrm{H} 23 \mathrm{~N} 3 \mathrm{O}$ & 357.1841 & FIU 0480 \\
\hline $\begin{array}{c}\text { JWH } 018 \text { benzimidazole } \\
\text { analog }\end{array}$ & $\mathrm{C} 23 \mathrm{H} 22 \mathrm{~N} 2 \mathrm{O}$ & 342.1732 & FIU 0481 \\
\hline $\begin{array}{c}\text { JWH } 018 \text { N-(1-ethylpropyl) } \\
\text { isomer }\end{array}$ & $\mathrm{C} 24 \mathrm{H} 23 \mathrm{NO}$ & 341.1780 & FIU 0482 \\
\hline $\begin{array}{l}\text { JWH } 018 \text { N-(4-oxo-pentyl) } \\
\text { metabolite }\end{array}$ & $\mathrm{C} 24 \mathrm{H} 21 \mathrm{NO} 2$ & 355.1572 & FIU 0483 \\
\hline $\begin{array}{l}\text { JWH } 018 \text { N-(5-hydroxypentyl) } \\
\text { metabolite }\end{array}$ & $\mathrm{C} 24 \mathrm{H} 23 \mathrm{NO} 2$ & 357.1729 & FIU 0484 \\
\hline $\begin{array}{c}\text { JWH } 018 \mathrm{~N}-(5 \text {-hydroxypentyl) } \\
\text { /-D-Glucuronide }\end{array}$ & $\mathrm{C} 30 \mathrm{H} 31 \mathrm{NO} 8$ & 533.2050 & FIU 0485 \\
\hline $\begin{array}{c}\text { JWH } 018 \text { N-pentanoic acid } \beta \text { - } \\
\text { D-Glucuronide }\end{array}$ & $\mathrm{C} 30 \mathrm{H} 29 \mathrm{NO} 9$ & 547.1842 & FIU 0486 \\
\hline $\begin{array}{c}\text { JWH } 018 \text { N-propanoic acid } \\
\text { metabolite }\end{array}$ & $\mathrm{C} 22 \mathrm{H} 17 \mathrm{NO} 3$ & 343.1208 & FIU 0487 \\
\hline $\begin{array}{c}\text { JWH } 019 \text { 5-hydroxyindole } \\
\text { metabolite }\end{array}$ & $\mathrm{C} 25 \mathrm{H} 25 \mathrm{NO} 2$ & 371.1885 & FIU 0488 \\
\hline $\begin{array}{c}\text { JWH } 019 \text { N-(2-fluorohexyl) } \\
\text { isomer }\end{array}$ & $\mathrm{C} 25 \mathrm{H} 24 \mathrm{FNO}$ & 373.1842 & FIU 0489 \\
\hline $\begin{array}{c}\text { JWH } 019 \text { N-(3-fluorohexyl) } \\
\text { isomer }\end{array}$ & $\mathrm{C} 25 \mathrm{H} 24 \mathrm{FNO}$ & 373.1842 & FIU 0490 \\
\hline $\begin{array}{c}\text { JWH } 019 \text { N-(4-fluorohexyl) } \\
\text { isomer }\end{array}$ & $\mathrm{C} 25 \mathrm{H} 24 \mathrm{FNO}$ & 373.1842 & FIU 0491 \\
\hline $\begin{array}{c}\text { JWH } 019 \text { N-(5-fluorohexyl) } \\
\text { isomer }\end{array}$ & $\mathrm{C} 25 \mathrm{H} 24 \mathrm{FNO}$ & 373.1842 & FIU 0492 \\
\hline $\begin{array}{l}\text { JWH } 019 \text { N-(5-hydroxyhexyl) } \\
\text { metabolite }\end{array}$ & $\mathrm{C} 25 \mathrm{H} 25 \mathrm{NO} 2$ & 371.1885 & FIU 0493 \\
\hline
\end{tabular}




\begin{tabular}{|c|c|c|c|}
\hline $\begin{array}{l}\text { JWH } 019 \text { N-(6-fluorohexyl) } \\
\text { isomer }\end{array}$ & $\mathrm{C} 25 \mathrm{H} 24 \mathrm{FNO}$ & 373.1842 & FIU 0494 \\
\hline $\begin{array}{l}\text { JWH } 019 \text { N-(6-hydroxyhexyl) } \\
\text { metabolite }\end{array}$ & $\mathrm{C} 25 \mathrm{H} 25 \mathrm{NO} 2$ & 371.1885 & FIU 0495 \\
\hline $\begin{array}{c}\text { JWH } 019 \mathrm{~N}-(6-h y d r o x y h e x y l) \\
\beta \text {-D-Glucuronide }\end{array}$ & C31H33NO8 & 547.2206 & FIU 0496 \\
\hline JWH 030 2-naphthoyl isomer & $\mathrm{C} 20 \mathrm{H} 21 \mathrm{NO}$ & 291.1623 & FIU 0497 \\
\hline JWH 031 2'-isomer & $\mathrm{C} 21 \mathrm{H} 23 \mathrm{NO}$ & 305.1780 & FIU 0498 \\
\hline JWH 071 & $\mathrm{C} 21 \mathrm{H} 17 \mathrm{NO}$ & 299.1310 & FIU 0499 \\
\hline $\begin{array}{l}\text { JWH } 073 \text { 2-hydroxyindole } \\
\text { metabolite }\end{array}$ & $\mathrm{C} 23 \mathrm{H} 21 \mathrm{NO} 2$ & 343.1572 & FIU 0500 \\
\hline $\begin{array}{l}\text { JWH } 073 \text { 4-hydroxyindole } \\
\text { metabolite }\end{array}$ & $\mathrm{C} 23 \mathrm{H} 21 \mathrm{NO} 2$ & 343.1572 & FIU 0501 \\
\hline $\begin{array}{c}\text { JWH } 0735 \text {-hydroxyindole } \\
\text { metabolite }\end{array}$ & $\mathrm{C} 23 \mathrm{H} 21 \mathrm{NO} 2$ & 343.1572 & FIU 0502 \\
\hline $\begin{array}{l}\text { JWH } 073 \text { 6-hydroxyindole } \\
\text { metabolite }\end{array}$ & $\mathrm{C} 23 \mathrm{H} 21 \mathrm{NO} 2$ & 343.1572 & FIU 0503 \\
\hline $\begin{array}{l}\text { JWH } 073 \text { 6-methoxyindole } \\
\text { analog }\end{array}$ & $\mathrm{C} 24 \mathrm{H} 23 \mathrm{NO} 2$ & 357.1729 & FIU 0504 \\
\hline $\begin{array}{l}\text { JWH } 073 \text { 7-hydroxyindole } \\
\text { metabolite }\end{array}$ & $\mathrm{C} 23 \mathrm{H} 21 \mathrm{NO} 2$ & 343.1572 & FIU 0505 \\
\hline $\begin{array}{l}\text { JWH } 073 \text { N-(2-hydroxybutyl) } \\
\text { metabolite }\end{array}$ & $\mathrm{C} 23 \mathrm{H} 21 \mathrm{NO} 2$ & 343.1572 & FIU 0506 \\
\hline $\begin{array}{l}\text { JWH } 073 \text { N-(4-hydroxybutyl) } \\
\text { metabolite }\end{array}$ & $\mathrm{C} 23 \mathrm{H} 21 \mathrm{NO} 2$ & 343.1572 & FIU 0507 \\
\hline $\begin{array}{c}\text { JWH } 073 \mathrm{~N} \text {-(4-hydroxybutyl) } \\
\beta \text {-D-Glucuronide }\end{array}$ & $\mathrm{C} 29 \mathrm{H} 29 \mathrm{NO} 8$ & 519.1893 & FIU 0508 \\
\hline $\begin{array}{l}\text { JWH } 073 \text { N-butanoic acid } \\
\text { metabolite }\end{array}$ & $\mathrm{C} 23 \mathrm{H} 19 \mathrm{NO} 3$ & 357.1365 & FIU 0509 \\
\hline $\begin{array}{c}( \pm)-J W H ~ 073 \mathrm{~N}-(3- \\
\text { hydroxybutyl) metabolite }\end{array}$ & $\mathrm{C} 23 \mathrm{H} 21 \mathrm{NO} 2$ & 343.1572 & FIU 0510 \\
\hline $\begin{array}{l}\text { (R)-(-)-JWH } 073 \mathrm{~N}-(3- \\
\text { hydroxybutyl) metabolite }\end{array}$ & $\mathrm{C} 23 \mathrm{H} 21 \mathrm{NO} 2$ & 343.1572 & FIU 0511 \\
\hline $\begin{array}{l}(\mathrm{S})-(+)-\mathrm{JWH} 073 \mathrm{~N}-(3- \\
\text { hydroxybutyl) metabolite }\end{array}$ & $\mathrm{C} 23 \mathrm{H} 21 \mathrm{NO} 2$ & 343.1572 & FIU 0512 \\
\hline JWH 080 & $\mathrm{C} 24 \mathrm{H} 23 \mathrm{NO} 2$ & 357.1729 & FIU 0513 \\
\hline $\begin{array}{l}\text { JWH 081 4-hydroxynaphthyl } \\
\text { metabolite }\end{array}$ & $\mathrm{C} 24 \mathrm{H} 23 \mathrm{NO} 2$ & 357.1729 & FIU 0514 \\
\hline $\begin{array}{l}\text { JWH } 081 \text { N-(4-hydroxypentyl) } \\
\text { metabolite }\end{array}$ & $\mathrm{C} 25 \mathrm{H} 25 \mathrm{NO} 3$ & 387.1834 & FIU 0515 \\
\hline $\begin{array}{l}\text { JWH } 081 \text { N-(5-hydroxypentyl) } \\
\text { metabolite }\end{array}$ & $\mathrm{C} 25 \mathrm{H} 25 \mathrm{NO} 3$ & 387.1834 & FIU 0516 \\
\hline $\begin{array}{c}\text { JWH } 081 \text { N-pentanoic acid } \\
\text { metabolite }\end{array}$ & $\mathrm{C} 25 \mathrm{H} 23 \mathrm{NO} 4$ & 401.1627 & FIU 0517 \\
\hline
\end{tabular}




\begin{tabular}{|c|c|c|c|}
\hline $\begin{array}{c}\text { JWH 081-N- } \\
\text { (cyclohexylmethyl) analog }\end{array}$ & $\mathrm{C} 27 \mathrm{H} 27 \mathrm{NO} 2$ & 397.2042 & FIU 0518 \\
\hline JWH 116 & $\mathrm{C} 26 \mathrm{H} 27 \mathrm{NO}$ & 369.2093 & FIU 0519 \\
\hline $\begin{array}{l}\text { JWH } 122 \text { N-(4-hydroxypentyl) } \\
\text { metabolite }\end{array}$ & $\mathrm{C} 25 \mathrm{H} 25 \mathrm{NO} 2$ & 371.1885 & FIU 0520 \\
\hline $\begin{array}{c}\text { JWH } 122 \text { N-(5-hydroxypentyl) } \\
\text { metabolite }\end{array}$ & $\mathrm{C} 25 \mathrm{H} 25 \mathrm{NO} 2$ & 371.1885 & FIU 0521 \\
\hline JWH 133 & $\mathrm{C} 22 \mathrm{H} 32 \mathrm{O}$ & 312.2453 & FIU 0522 \\
\hline JWH 145 2-phenyl isomer & $\mathrm{C} 26 \mathrm{H} 25 \mathrm{NO}$ & 367.1936 & FIU 0523 \\
\hline JWH 146 & $\mathrm{C} 28 \mathrm{H} 29 \mathrm{NO}$ & 395.2249 & FIU 0524 \\
\hline JWH 149 & $\mathrm{C} 26 \mathrm{H} 27 \mathrm{NO}$ & 369.2093 & FIU 0525 \\
\hline JWH 167 & $\mathrm{C} 21 \mathrm{H} 23 \mathrm{NO}$ & 305.1780 & FIU 0526 \\
\hline JWH 176 & $\mathrm{C} 25 \mathrm{H} 24$ & 324.1878 & FIU 0527 \\
\hline JWH 193 & $\mathrm{C} 26 \mathrm{H} 26 \mathrm{~N} 2 \mathrm{O} 2$ & 398.1994 & FIU 0528 \\
\hline JWH 198 & $\mathrm{C} 26 \mathrm{H} 26 \mathrm{~N} 2 \mathrm{O} 3$ & 414.1943 & FIU 0529 \\
\hline $\begin{array}{c}\text { JWH } 200 \text { 4-hydroxyindole } \\
\text { metabolite }\end{array}$ & $\mathrm{C} 25 \mathrm{H} 24 \mathrm{~N} 2 \mathrm{O} 3$ & 400.1787 & FIU 0530 \\
\hline $\begin{array}{c}\text { JWH } 200 \text { 5-hydroxyindole } \\
\text { metabolite }\end{array}$ & $\mathrm{C} 25 \mathrm{H} 24 \mathrm{~N} 2 \mathrm{O} 3$ & 400.1787 & FIU 0531 \\
\hline $\begin{array}{c}\text { JWH } 200 \text { 6-hydroxyindole } \\
\text { metabolite }\end{array}$ & $\mathrm{C} 25 \mathrm{H} 24 \mathrm{~N} 2 \mathrm{O} 3$ & 400.1787 & FIU 0532 \\
\hline $\begin{array}{l}\text { JWH } 200 \text { 7-hydroxyindole } \\
\text { metabolite }\end{array}$ & $\mathrm{C} 25 \mathrm{H} 24 \mathrm{~N} 2 \mathrm{O} 3$ & 400.1787 & FIU 0533 \\
\hline JWH 203 & $\mathrm{C} 21 \mathrm{H} 22 \mathrm{ClNO}$ & 339.1390 & FIU 0534 \\
\hline $\begin{array}{c}\text { JWH } 203 \text { N-(4-hydroxypentyl) } \\
\text { metabolite }\end{array}$ & $\mathrm{C} 21 \mathrm{H} 22 \mathrm{CINO} 2$ & 355.1339 & FIU 0535 \\
\hline $\begin{array}{c}\text { JWH } 203 \text { N-(5-hydroxypentyl) } \\
\text { metabolite }\end{array}$ & $\mathrm{C} 21 \mathrm{H} 22 \mathrm{CINO} 2$ & 355.1339 & FIU 0536 \\
\hline $\begin{array}{c}\text { JWH } 203 \text { N-pentanoic acid } \\
\text { metabolite }\end{array}$ & $\mathrm{C} 21 \mathrm{H} 20 \mathrm{CINO} 3$ & 369.1132 & FIU 0537 \\
\hline $\begin{array}{c}\text { JWH } 210 \text { 2-ethylnaphthyl } \\
\text { isomer }\end{array}$ & $\mathrm{C} 26 \mathrm{H} 27 \mathrm{NO}$ & 369.2093 & FIU 0538 \\
\hline $\begin{array}{c}\text { JWH } 210 \text { 5-hydroxyindole } \\
\text { metabolite }\end{array}$ & $\mathrm{C} 26 \mathrm{H} 27 \mathrm{NO} 2$ & 385.2042 & FIU 0539 \\
\hline $\begin{array}{c}\text { JWH } 210 \text { N-(4-hydroxypentyl) } \\
\text { metabolite }\end{array}$ & $\mathrm{C} 26 \mathrm{H} 27 \mathrm{NO} 2$ & 385.2042 & FIU 0540 \\
\hline $\begin{array}{l}\text { JWH } 210 \text { N-(5-hydroxypentyl) } \\
\text { metabolite }\end{array}$ & $\mathrm{C} 26 \mathrm{H} 27 \mathrm{NO} 2$ & 385.2042 & FIU 0541 \\
\hline $\begin{array}{c}\text { JWH } 210 \text { N-pentanoic acid } \\
\text { metabolite }\end{array}$ & $\mathrm{C} 26 \mathrm{H} 25 \mathrm{NO} 3$ & 399.1834 & FIU 0542 \\
\hline JWH 213 & $\mathrm{C} 27 \mathrm{H} 29 \mathrm{NO}$ & 383.2249 & FIU 0543 \\
\hline $\begin{array}{c}\text { JWH } 250 \text { 5-hydroxyindole } \\
\text { metabolite }\end{array}$ & $\mathrm{C} 22 \mathrm{H} 25 \mathrm{NO} 3$ & 351.1834 & FIU 0544 \\
\hline
\end{tabular}




\begin{tabular}{|c|c|c|c|}
\hline $\begin{array}{l}\text { JWH } 250 \text { N-(4-hydroxypentyl) } \\
\text { metabolite }\end{array}$ & $\mathrm{C} 22 \mathrm{H} 25 \mathrm{NO} 3$ & 351.1834 & FIU 0545 \\
\hline $\begin{array}{l}\text { JWH } 250 \text { N-(5-hydroxypentyl) } \\
\text { metabolite }\end{array}$ & $\mathrm{C} 22 \mathrm{H} 25 \mathrm{NO} 3$ & 351.1834 & FIU 0546 \\
\hline $\begin{array}{c}\text { JWH } 250 \text { N-pentanoic acid } \\
\text { metabolite }\end{array}$ & $\mathrm{C} 22 \mathrm{H} 23 \mathrm{NO} 4$ & 365.1627 & FIU 0547 \\
\hline JWH 307 5'-isomer & $\mathrm{C} 25 \mathrm{H} 24 \mathrm{FNO}$ & 373.1842 & FIU 0548 \\
\hline JWH 309 5'-isomer & $\mathrm{C} 30 \mathrm{H} 27 \mathrm{NO}$ & 417.2093 & FIU 0549 \\
\hline JWH 387 & $\mathrm{C} 24 \mathrm{H} 22 \mathrm{BrNO}$ & 419.0885 & FIU 0550 \\
\hline $\begin{array}{l}\text { JWH } 398 \text { N-(4-hydroxypentyl) } \\
\text { metabolite }\end{array}$ & $\mathrm{C} 24 \mathrm{H} 22 \mathrm{ClNO} 2$ & 391.1339 & FIU 0551 \\
\hline $\begin{array}{c}\text { JWH } 398 \text { N-(5-hydroxypentyl) } \\
\text { metabolite }\end{array}$ & C24H22CINO2 & 391.1339 & FIU 0552 \\
\hline $\begin{array}{c}\text { JWH } 398 \text { N-pentanoic acid } \\
\text { metabolite }\end{array}$ & C24H20CINO3 & 405.1132 & FIU 0553 \\
\hline JWH 412 & $\mathrm{C} 24 \mathrm{H} 22 \mathrm{FNO}$ & 359.1685 & FIU 0554 \\
\hline (-)-CP 47,497 & $\mathrm{C} 21 \mathrm{H} 34 \mathrm{O} 2$ & 318.2559 & FIU 0555 \\
\hline$(-)-C P ~ 55,940$ & $\mathrm{C} 24 \mathrm{H} 40 \mathrm{O} 3$ & 376.2977 & FIU 0556 \\
\hline$(+)-\mathrm{CP} 47,497$ & $\mathrm{C} 21 \mathrm{H} 34 \mathrm{O} 2$ & 318.2559 & FIU 0557 \\
\hline$(+)-C P ~ 55,940$ & $\mathrm{C} 24 \mathrm{H} 40 \mathrm{O} 3$ & 376.2977 & FIU 0558 \\
\hline $\begin{array}{c}\text { ( } \pm \text { )3-epi CP 47,497-C8- } \\
\text { homolog }\end{array}$ & $\mathrm{C} 22 \mathrm{H} 36 \mathrm{O} 2$ & 332.2715 & FIU 0559 \\
\hline ( \pm 5-epi CP 55,940 & $\mathrm{C} 24 \mathrm{H} 40 \mathrm{O} 3$ & 376.2977 & FIU 0560 \\
\hline$( \pm)-\mathrm{CP} 47,497$ & $\mathrm{C} 21 \mathrm{H} 34 \mathrm{O} 2$ & 318.2559 & FIU 0561 \\
\hline $\begin{array}{c}( \pm) \text {-CP 47,497-C7-hydroxy } \\
\text { metabolite }\end{array}$ & $\mathrm{C} 21 \mathrm{H} 34 \mathrm{O} 3$ & 334.2508 & FIU 0562 \\
\hline$( \pm)$-CP 47,497-C8-homolog & $\mathrm{C} 22 \mathrm{H} 36 \mathrm{O} 2$ & 332.2715 & FIU 0563 \\
\hline$( \pm)-\mathrm{CP} 55,940$ & $\mathrm{C} 24 \mathrm{H} 40 \mathrm{O} 3$ & 376.2977 & FIU 0564 \\
\hline ( \pm )-epi CP 47,497 & $\mathrm{C} 21 \mathrm{H} 34 \mathrm{O} 2$ & 318.2559 & FIU 0565 \\
\hline CP 47,497-C6-homolog & $\mathrm{C} 20 \mathrm{H} 32 \mathrm{O} 2$ & 304.2402 & FIU 0566 \\
\hline $\begin{array}{c}\text { CP 47,497-C8-homolog C-8- } \\
\text { hydroxy metabolite }\end{array}$ & $\mathrm{C} 22 \mathrm{H} 36 \mathrm{O} 3$ & 348.2664 & FIU 0567 \\
\hline CP 47,497-C9-homolog & $\mathrm{C} 23 \mathrm{H} 38 \mathrm{O} 2$ & 346.2872 & FIU 0568 \\
\hline CP 47,497-para-quinone analog & $\mathrm{C} 21 \mathrm{H} 32 \mathrm{O} 3$ & 332.2351 & FIU 0569 \\
\hline $\begin{array}{l}\text { RCS-4 4-hydroxyphenyl } \\
\text { metabolite }\end{array}$ & $\mathrm{C} 20 \mathrm{H} 21 \mathrm{NO} 2$ & 307.1572 & FIU 0570 \\
\hline RCS-4 M10 metabolite & $\mathrm{C} 20 \mathrm{H} 21 \mathrm{NO} 3$ & 323.1521 & FIU 0571 \\
\hline RCS-4 M11 metabolite & $\mathrm{C} 20 \mathrm{H} 19 \mathrm{NO} 3$ & 321.1365 & FIU 0572 \\
\hline RCS-4 M9 metabolite & $\mathrm{C} 20 \mathrm{H} 21 \mathrm{NO} 3$ & 323.1521 & FIU 0573 \\
\hline $\begin{array}{l}\text { RCS-4 N-(4-hydroxypentyl) } \\
\text { metabolite }\end{array}$ & $\mathrm{C} 21 \mathrm{H} 23 \mathrm{NO} 3$ & 337.1678 & FIU 0574 \\
\hline
\end{tabular}




\begin{tabular}{|c|c|c|c|}
\hline $\begin{array}{c}\text { RCS-4 N-(5-carboxypentyl) } \\
\text { metabolite }\end{array}$ & $\mathrm{C} 21 \mathrm{H} 21 \mathrm{NO} 4$ & 351.1471 & FIU 0575 \\
\hline $\begin{array}{c}\text { RCS-4 N-(5-hydroxypentyl) } \\
\text { metabolite }\end{array}$ & $\mathrm{C} 21 \mathrm{H} 23 \mathrm{NO} 3$ & 337.1678 & FIU 0576 \\
\hline 5-fluoro NPB-22 & C22H20FN3O2 & 377.1540 & FIU 0577 \\
\hline 5-fluoro PB-22 & C23H21FN2O2 & 376.1587 & FIU 0578 \\
\hline $\begin{array}{c}\text { 5-fluoro PB-22 3- } \\
\text { carboxyindole metabolite }\end{array}$ & C14H16FNO2 & 249.1165 & FIU 0579 \\
\hline $\begin{array}{c}\text { 5-fluoro PB-22 3- } \\
\text { hydroxyquinoline isomer }\end{array}$ & C21H21FN2O2 & 352.1587 & FIU 0580 \\
\hline $\begin{array}{c}\text { 5-fluoro PB-22 4- } \\
\text { hydroxyisoquinoline isomer }\end{array}$ & $\mathrm{C} 23 \mathrm{H} 21 \mathrm{FN} 2 \mathrm{O} 2$ & 376.1587 & FIU 0581 \\
\hline $\begin{array}{c}\text { 5-fluoro PB-22 4- } \\
\text { hydroxyquinoline isomer }\end{array}$ & $\mathrm{C} 23 \mathrm{H} 21 \mathrm{FN} 2 \mathrm{O} 2$ & 376.1587 & FIU 0582 \\
\hline $\begin{array}{c}\text { 5-fluoro PB-22 5- } \\
\text { hydroxyisoquinoline isomer }\end{array}$ & $\mathrm{C} 23 \mathrm{H} 21 \mathrm{FN} 2 \mathrm{O} 2$ & 376.1587 & FIU 0583 \\
\hline $\begin{array}{c}\text { 5-fluoro PB-22 5- } \\
\text { hydroxyquinoline isomer }\end{array}$ & $\mathrm{C} 23 \mathrm{H} 21 \mathrm{FN} 2 \mathrm{O} 2$ & 376.1587 & FIU 0584 \\
\hline $\begin{array}{c}\text { 5-fluoro PB-22 6- } \\
\text { hydroxyisoquinoline isomer }\end{array}$ & $\mathrm{C} 23 \mathrm{H} 21 \mathrm{FN} 2 \mathrm{O} 2$ & 376.1587 & FIU 0585 \\
\hline $\begin{array}{c}\text { 5-fluoro PB-22 6- } \\
\text { hydroxyquinoline isomer }\end{array}$ & $\mathrm{C} 23 \mathrm{H} 21 \mathrm{FN} 2 \mathrm{O} 2$ & 376.1587 & FIU 0586 \\
\hline $\begin{array}{c}\text { 5-fluoro PB-22 7- } \\
\text { hydroxyisoquinoline isomer }\end{array}$ & $\mathrm{C} 23 \mathrm{H} 21 \mathrm{FN} 2 \mathrm{O} 2$ & 376.1587 & FIU 0587 \\
\hline $\begin{array}{c}\text { 5-fluoro PB-22 7- } \\
\text { hydroxyquinoline isomer }\end{array}$ & $\mathrm{C} 23 \mathrm{H} 21 \mathrm{FN} 2 \mathrm{O} 2$ & 376.1587 & FIU 0588 \\
\hline $\begin{array}{c}\text { 5-fluoro PB-22 8- } \\
\text { hydroxyisoquinoline isomer }\end{array}$ & $\mathrm{C} 23 \mathrm{H} 21 \mathrm{FN} 2 \mathrm{O} 2$ & 376.1587 & FIU 0589 \\
\hline $\begin{array}{l}\text { 5-fluoro PB-22 N-(2- } \\
\text { fluoropentyl) isomer }\end{array}$ & $\mathrm{C} 23 \mathrm{H} 21 \mathrm{FN} 2 \mathrm{O} 2$ & 376.1587 & FIU 0590 \\
\hline $\begin{array}{l}\text { 5-fluoro PB-22 N-(3- } \\
\text { fluoropentyl) isomer }\end{array}$ & $\mathrm{C} 23 \mathrm{H} 21 \mathrm{FN} 2 \mathrm{O} 2$ & 376.1587 & FIU 0591 \\
\hline $\begin{array}{l}\text { 5-fluoro PB-22 N-(4- } \\
\text { fluoropentyl) isomer }\end{array}$ & $\mathrm{C} 23 \mathrm{H} 21 \mathrm{FN} 2 \mathrm{O} 2$ & 376.1587 & FIU 0592 \\
\hline FDU-PB-22 & C26H18FNO2 & 395.1322 & FIU 0593 \\
\hline FUB-PB-22 & C25H17FN2O2 & 396.1274 & FIU 0594 \\
\hline NPB-22 & $\mathrm{C} 22 \mathrm{H} 21 \mathrm{~N} 3 \mathrm{O} 2$ & 359.1634 & FIU 0595 \\
\hline PB-22 & $\mathrm{C} 23 \mathrm{H} 22 \mathrm{~N} 2 \mathrm{O} 2$ & 358.1681 & FIU 0596 \\
\hline $\begin{array}{l}\text { PB-22 3-carboxyindole } \\
\text { metabolite }\end{array}$ & $\mathrm{C} 14 \mathrm{H} 17 \mathrm{NO} 2$ & 231.1259 & FIU 0597 \\
\hline $\begin{array}{c}\text { PB-22 3-hydroxyquinoline } \\
\text { isomer }\end{array}$ & $\mathrm{C} 23 \mathrm{H} 22 \mathrm{~N} 2 \mathrm{O} 2$ & 358.1681 & FIU 0598 \\
\hline $\begin{array}{c}\text { PB-22 4-hydroxyisoquinoline } \\
\text { isomer }\end{array}$ & $\mathrm{C} 23 \mathrm{H} 22 \mathrm{~N} 2 \mathrm{O} 2$ & 358.1681 & FIU 0599 \\
\hline
\end{tabular}




\begin{tabular}{|c|c|c|c|}
\hline $\begin{array}{l}\text { PB-22 4-hydroxyquinoline } \\
\text { isomer }\end{array}$ & $\mathrm{C} 23 \mathrm{H} 22 \mathrm{~N} 2 \mathrm{O} 2$ & 358.1681 & FIU 0600 \\
\hline $\begin{array}{c}\text { PB-22 5-hydroxyisoquinoline } \\
\text { isomer }\end{array}$ & $\mathrm{C} 23 \mathrm{H} 22 \mathrm{~N} 2 \mathrm{O} 2$ & 358.1681 & FIU 0601 \\
\hline $\begin{array}{c}\text { PB-22 5-hydroxyquinoline } \\
\text { isomer }\end{array}$ & $\mathrm{C} 23 \mathrm{H} 22 \mathrm{~N} 2 \mathrm{O} 2$ & 358.1681 & FIU 0602 \\
\hline $\begin{array}{l}\text { PB-22 6-hydroxyisoquinoline } \\
\text { isomer }\end{array}$ & $\mathrm{C} 23 \mathrm{H} 22 \mathrm{~N} 2 \mathrm{O} 2$ & 358.1681 & FIU 0603 \\
\hline $\begin{array}{c}\text { PB-22 6-hydroxyquinoline } \\
\text { isomer }\end{array}$ & $\mathrm{C} 23 \mathrm{H} 22 \mathrm{~N} 2 \mathrm{O} 2$ & 358.1681 & FIU 0604 \\
\hline $\begin{array}{l}\text { PB-22 7-hydroxyisoquinoline } \\
\text { isomer }\end{array}$ & $\mathrm{C} 23 \mathrm{H} 22 \mathrm{~N} 2 \mathrm{O} 2$ & 358.1681 & FIU 0605 \\
\hline $\begin{array}{l}\text { PB-22 7-hydroxyquinoline } \\
\text { isomer }\end{array}$ & $\mathrm{C} 23 \mathrm{H} 22 \mathrm{~N} 2 \mathrm{O} 2$ & 358.1681 & FIU 0606 \\
\hline $\begin{array}{c}\text { PB-22 8-hydroxyisoquinoline } \\
\text { isomer }\end{array}$ & $\mathrm{C} 23 \mathrm{H} 22 \mathrm{~N} 2 \mathrm{O} 2$ & 358.1681 & FIU 0607 \\
\hline $\begin{array}{l}\text { PB-22 N-(4-hydroxypentyl) } \\
\text { metabolite }\end{array}$ & $\mathrm{C} 23 \mathrm{H} 22 \mathrm{~N} 2 \mathrm{O} 3$ & 374.1630 & FIU 0608 \\
\hline $\begin{array}{l}\text { PB-22 N-(4-hydroxypentyl)-3- } \\
\text { carboxyindole metabolite }\end{array}$ & $\mathrm{C} 14 \mathrm{H} 17 \mathrm{NO} 3$ & 347.1208 & FIU 0609 \\
\hline $\begin{array}{l}\text { PB-22 N-(5-hydroxypentyl) } \\
\text { metabolite }\end{array}$ & $\mathrm{C} 23 \mathrm{H} 22 \mathrm{~N} 2 \mathrm{O} 3$ & 374.1630 & FIU 0610 \\
\hline $\begin{array}{l}\text { PB-22 N-(5-hydroxypentyl)-3- } \\
\text { carboxyindole metabolite }\end{array}$ & $\mathrm{C} 14 \mathrm{H} 17 \mathrm{NO} 3$ & 247.1208 & FIU 0611 \\
\hline $\begin{array}{l}\text { PB-22 N-pentanoic acid } \\
\text { metabolite }\end{array}$ & $\mathrm{C} 23 \mathrm{H} 20 \mathrm{~N} 2 \mathrm{O} 4$ & 388.1423 & FIU 0612 \\
\hline $\begin{array}{l}\text { PB-22 N-pentanoic acid-3- } \\
\text { carboxyindole metabolite }\end{array}$ & $\mathrm{C} 14 \mathrm{H} 15 \mathrm{NO} 4$ & 261.1001 & FIU 0613 \\
\hline $\begin{array}{l}\text { AM2201 8-quinolinyl } \\
\text { carboxamide }\end{array}$ & $\mathrm{C} 23 \mathrm{H} 22 \mathrm{FN} 3 \mathrm{O}$ & 375.1747 & FIU 0614 \\
\hline BB-22 & $\mathrm{C} 25 \mathrm{H} 24 \mathrm{~N} 2 \mathrm{O} 2$ & 384.1838 & FIU 0615 \\
\hline $\begin{array}{l}\text { BB-22 3-carboxyindole } \\
\text { metabolite }\end{array}$ & $\mathrm{C} 16 \mathrm{H} 19 \mathrm{NO} 2$ & 257.1416 & FIU 0616 \\
\hline $\begin{array}{l}\text { BB-22 3-hydroxyquinoline } \\
\text { isomer }\end{array}$ & $\mathrm{C} 25 \mathrm{H} 24 \mathrm{~N} 2 \mathrm{O} 2$ & 384.1838 & FIU 0617 \\
\hline $\begin{array}{l}\text { BB-22 4-hydroxyisoquinoline } \\
\text { isomer }\end{array}$ & $\mathrm{C} 25 \mathrm{H} 24 \mathrm{~N} 2 \mathrm{O} 2$ & 384.1838 & FIU 0618 \\
\hline $\begin{array}{l}\text { BB-22 4-hydroxyquinoline } \\
\text { isomer }\end{array}$ & $\mathrm{C} 25 \mathrm{H} 24 \mathrm{~N} 2 \mathrm{O} 2$ & 384.1838 & FIU 0619 \\
\hline $\begin{array}{l}\text { BB-22 5-hydroxyisoquinoline } \\
\text { isomer }\end{array}$ & $\mathrm{C} 25 \mathrm{H} 24 \mathrm{~N} 2 \mathrm{O} 2$ & 384.1838 & FIU 0620 \\
\hline $\begin{array}{l}\text { BB-22 5-hydroxyquinoline } \\
\text { isomer }\end{array}$ & $\mathrm{C} 25 \mathrm{H} 24 \mathrm{~N} 2 \mathrm{O} 2$ & 384.1838 & FIU 0621 \\
\hline
\end{tabular}




\begin{tabular}{|c|c|c|c|}
\hline $\begin{array}{c}\text { BB-22 6-hydroxyisoquinoline } \\
\text { isomer }\end{array}$ & $\mathrm{C} 25 \mathrm{H} 24 \mathrm{~N} 2 \mathrm{O} 2$ & 384.1838 & FIU 0622 \\
\hline $\begin{array}{c}\text { BB-22 6-hydroxyquinoline } \\
\text { isomer }\end{array}$ & $\mathrm{C} 25 \mathrm{H} 24 \mathrm{~N} 2 \mathrm{O} 2$ & 384.1838 & FIU 0623 \\
\hline $\begin{array}{c}\text { BB-22 7-hydroxyisoquinoline } \\
\text { isomer }\end{array}$ & $\mathrm{C} 25 \mathrm{H} 24 \mathrm{~N} 2 \mathrm{O} 2$ & 384.1838 & FIU 0624 \\
\hline $\begin{array}{l}\text { BB-22 7-hydroxyquinoline } \\
\text { isomer }\end{array}$ & $\mathrm{C} 25 \mathrm{H} 24 \mathrm{~N} 2 \mathrm{O} 2$ & 384.1838 & FIU 0625 \\
\hline $\begin{array}{c}\text { BB-22 8-hydroxyisoquinoline } \\
\text { isomer }\end{array}$ & $\mathrm{C} 25 \mathrm{H} 24 \mathrm{~N} 2 \mathrm{O} 2$ & 384.1838 & FIU 0626 \\
\hline AM1220 azepane isomer & $\mathrm{C} 26 \mathrm{H} 26 \mathrm{~N} 2 \mathrm{O}$ & 382.2045 & FIU 0627 \\
\hline $\begin{array}{l}\text { AM2201 2-hydroxyindole } \\
\text { metabolite }\end{array}$ & $\mathrm{C} 24 \mathrm{H} 22 \mathrm{FNO} 2$ & 375.1635 & FIU 0628 \\
\hline $\begin{array}{l}\text { AM2201 5-hydroxyindole } \\
\text { metabolite }\end{array}$ & $\mathrm{C} 24 \mathrm{H} 22 \mathrm{FNO} 2$ & 375.1635 & FIU 0629 \\
\hline $\begin{array}{l}\text { AM2201 6-hydroxyindole } \\
\text { metabolite }\end{array}$ & $\mathrm{C} 24 \mathrm{H} 22 \mathrm{FNO} 2$ & 375.1635 & FIU 0630 \\
\hline $\begin{array}{l}\text { AM2201 7-hydroxyindole } \\
\text { metabolite }\end{array}$ & $\mathrm{C} 24 \mathrm{H} 22 \mathrm{FNO} 2$ & 375.1635 & FIU 0631 \\
\hline $\begin{array}{l}\text { AM2201 N-(4-hydroxypentyl) } \\
\text { metabolite }\end{array}$ & $\mathrm{C} 24 \mathrm{H} 22 \mathrm{FNO} 2$ & 375.1635 & FIU 0632 \\
\hline AM2233 azepane isomer & $\mathrm{C} 22 \mathrm{H} 23 \mathrm{IN} 2 \mathrm{O}$ & 458.0855 & FIU 0633 \\
\hline EAM2201 & $\mathrm{C} 26 \mathrm{H} 26 \mathrm{FNO}$ & 387.1998 & FIU 0634 \\
\hline NM2201 & $\mathrm{C} 24 \mathrm{H} 22 \mathrm{FNO} 2$ & 375.1635 & FIU 0635 \\
\hline $\begin{array}{l}\text { AM2201 N-(3-chloropentyl) } \\
\text { isomer }\end{array}$ & $\mathrm{C} 24 \mathrm{H} 22 \mathrm{CINO}$ & 375.1390 & FIU 0636 \\
\hline $\begin{array}{c}\text { MAM2201 N-(2-fluoropentyl) } \\
\text { isomer }\end{array}$ & $\mathrm{C} 25 \mathrm{H} 24 \mathrm{FNO}$ & 373.1842 & FIU 0637 \\
\hline $\begin{array}{c}\text { MAM2201 N-(3-fluoropentyl) } \\
\text { isomer }\end{array}$ & $\mathrm{C} 25 \mathrm{H} 24 \mathrm{FNO}$ & 373.1842 & FIU 0638 \\
\hline $\begin{array}{c}\text { MAM2201 N-(4-fluoropentyl) } \\
\text { isomer }\end{array}$ & $\mathrm{C} 25 \mathrm{H} 24 \mathrm{FNO}$ & 373.1842 & FIU 0639 \\
\hline $\begin{array}{c}\text { MAM2201 N-(4- } \\
\text { hydroxypentyl) metabolite }\end{array}$ & $\mathrm{C} 25 \mathrm{H} 24 \mathrm{FNO} 2$ & 389.1791 & FIU 0640 \\
\hline $\begin{array}{l}\text { MAM2201 N-(5-chloropentyl) } \\
\text { analog }\end{array}$ & $\mathrm{C} 25 \mathrm{H} 24 \mathrm{ClNO}$ & 389.1546 & FIU 0641 \\
\hline $\begin{array}{l}\text { MAM2201 N-(5-chloropentyl) } \\
\text { analog-d5 }\end{array}$ & C25H19D5CINO & 394.1860 & FIU 0642 \\
\hline $\begin{array}{c}\text { MAM2201 N-pentanoic acid } \\
\text { metabolite }\end{array}$ & $\mathrm{C} 25 \mathrm{H} 23 \mathrm{NO} 3$ & 385.1678 & FIU 0643 \\
\hline $\begin{array}{c}( \pm)-U R-144 \mathrm{~N}-(4- \\
\text { hydroxypentyl) metabolite }\end{array}$ & $\mathrm{C} 21 \mathrm{H} 29 \mathrm{NO} 2$ & 327.2198 & FIU 0644 \\
\hline UR-144 Degradant & $\mathrm{C} 21 \mathrm{H} 29 \mathrm{NO}$ & 311.2249 & FIU 0645 \\
\hline
\end{tabular}




\begin{tabular}{|c|c|c|c|}
\hline $\begin{array}{l}\text { UR-144 Degradant N- } \\
\text { pentanoic acid metabolite }\end{array}$ & $\mathrm{C} 21 \mathrm{H} 27 \mathrm{NO} 3$ & 341.1991 & FIU 0646 \\
\hline $\begin{array}{l}\text { UR-144 N-(2-chloropentyl) } \\
\text { analog }\end{array}$ & $\mathrm{C} 21 \mathrm{H} 28 \mathrm{CINO}$ & 345.1859 & FIU 0647 \\
\hline $\begin{array}{l}\text { UR-144 N-(2-hydroxypentyl) } \\
\text { metabolite } \\
\end{array}$ & $\mathrm{C} 21 \mathrm{H} 29 \mathrm{NO} 2$ & 327.2198 & FIU 0648 \\
\hline $\begin{array}{l}\text { UR-144 N-(3-chloropentyl) } \\
\text { analog }\end{array}$ & $\mathrm{C} 21 \mathrm{H} 28 \mathrm{CINO}$ & 345.1859 & FIU 0649 \\
\hline $\begin{array}{l}\text { UR-144 N-(4-chloropentyl) } \\
\text { analog }\end{array}$ & $\mathrm{C} 21 \mathrm{H} 28 \mathrm{CINO}$ & 345.1859 & FIU 0650 \\
\hline $\begin{array}{l}\text { UR-144 N-(5-bromopentyl) } \\
\text { analog }\end{array}$ & $\mathrm{C} 21 \mathrm{H} 28 \mathrm{BrNO}$ & 389.1354 & FIU 0651 \\
\hline $\begin{array}{l}\text { UR-144 N-(5-chloropentyl) } \\
\text { analog }\end{array}$ & $\mathrm{C} 21 \mathrm{H} 28 \mathrm{CINO}$ & 345.1859 & FIU 0652 \\
\hline $\begin{array}{l}\text { UR-144 N-(5-hydroxypentyl) } \\
\text { metabolite }\end{array}$ & $\mathrm{C} 21 \mathrm{H} 29 \mathrm{NO} 2$ & 327.2198 & FIU 0653 \\
\hline $\begin{array}{c}\text { UR-144 N-(5-hydroxypentyl) } \\
\beta \text {-D-Glucuronide }\end{array}$ & C27H37NO8 & 503.2519 & FIU 0654 \\
\hline $\begin{array}{l}\text { UR-144 N-pentanoic acid } \\
\text { metabolite }\end{array}$ & $\mathrm{C} 21 \mathrm{H} 27 \mathrm{NO} 3$ & 341.1991 & FIU 0655 \\
\hline $\begin{array}{l}\text { UR-144 N-(5-methylhexyl) } \\
\text { analog }\end{array}$ & C23H33NO & 339.2562 & FIU 0656 \\
\hline UR-144 N-heptyl analog & $\mathrm{C} 23 \mathrm{H} 33 \mathrm{NO}$ & 339.2562 & FIU 0657 \\
\hline FUB-144 & $\mathrm{C} 23 \mathrm{H} 24 \mathrm{FNO}$ & 349.1842 & FIU 0658 \\
\hline $\begin{array}{l}\text { XLR11 6-hydroxyindole } \\
\text { metabolite }\end{array}$ & C21H28FNO2 & 345.2104 & FIU 0659 \\
\hline XLR11 Degradant & $\mathrm{C} 21 \mathrm{H} 28 \mathrm{FNO}$ & 329.2155 & FIU 0660 \\
\hline $\begin{array}{l}\text { XLR11 N-(2-fluoropentyl) } \\
\text { isomer }\end{array}$ & $\mathrm{C} 21 \mathrm{H} 28 \mathrm{FNO}$ & 329.2155 & FIU 0661 \\
\hline $\begin{array}{l}\text { XLR11 N-(3-fluoropentyl) } \\
\text { isomer }\end{array}$ & $\mathrm{C} 21 \mathrm{H} 28 \mathrm{FNO}$ & 329.2155 & FIU 0662 \\
\hline $\begin{array}{l}\text { XLR11 N-(4-fluoropentyl) } \\
\text { isomer }\end{array}$ & $\mathrm{C} 21 \mathrm{H} 28 \mathrm{FNO}$ & 329.2155 & FIU 0663 \\
\hline $\begin{array}{l}\text { XLR11 N-(4-hydroxypentyl) } \\
\text { metabolite }\end{array}$ & $\mathrm{C} 21 \mathrm{H} 28 \mathrm{FNO} 2$ & 345.2104 & FIU 0664 \\
\hline XLR11 N-(4-pentenyl) analog & $\mathrm{C} 21 \mathrm{H} 27 \mathrm{NO}$ & 309.2093 & FIU 0665 \\
\hline XLR12 & $\mathrm{C} 20 \mathrm{H} 24 \mathrm{~F} 3 \mathrm{NO}$ & 351.1810 & FIU 0666 \\
\hline Acetyl fentanyl & $\mathrm{C} 21 \mathrm{H} 26 \mathrm{~N} 2 \mathrm{O}$ & 322.2045 & FIU 0667 \\
\hline Acetyl norfentanyl & C13H18N2O & 218.1419 & FIU 0668 \\
\hline Butyryl fentanyl & $\mathrm{C} 23 \mathrm{H} 30 \mathrm{~N} 2 \mathrm{O}$ & 350.2358 & FIU 0669 \\
\hline para-Fluorofentanyl & $\mathrm{C} 22 \mathrm{H} 27 \mathrm{FN} 2 \mathrm{O}$ & 354.2107 & FIU 0670 \\
\hline AH 7921 & $\mathrm{C} 16 \mathrm{H} 22 \mathrm{Cl} 2 \mathrm{~N} 2 \mathrm{O}$ & 328.1109 & FIU 0671 \\
\hline ATM4 4-acetoxy analog & $\mathrm{C} 23 \mathrm{H} 25 \mathrm{NO} 5$ & 395.1733 & FIU 0672 \\
\hline
\end{tabular}




\begin{tabular}{|c|c|c|c|}
\hline 3-hydroxy Phenazepam & $\mathrm{C} 15 \mathrm{H} 10 \mathrm{BrClN} 2 \mathrm{O} 2$ & 363.9614 & FIU 0673 \\
\hline Bromazepam & $\mathrm{C} 14 \mathrm{H} 10 \mathrm{BrN} 3 \mathrm{O}$ & 315.0007 & FIU 0674 \\
\hline Delorazepam & $\mathrm{C} 15 \mathrm{H} 10 \mathrm{Cl} 2 \mathrm{~N} 2 \mathrm{O}$ & 304.0170 & FIU 0675 \\
\hline Diclazepam & $\mathrm{C} 16 \mathrm{H} 12 \mathrm{Cl} 2 \mathrm{~N} 2 \mathrm{O}$ & 318.0327 & FIU 0676 \\
\hline Etizolam & C17H15CIN4S & 342.0706 & FIU 0677 \\
\hline Flubromazepam & C15H10BrFN2O & 331.9961 & FIU 0678 \\
\hline MCOPPB & $\mathrm{C} 26 \mathrm{H} 40 \mathrm{~N} 4$ & 408.3253 & FIU 0679 \\
\hline Pyrazolam & $\mathrm{C} 16 \mathrm{H} 12 \mathrm{BrN5}$ & 353.0276 & FIU 0680 \\
\hline 1'-naphthoyl-2-methylindole & $\mathrm{C} 20 \mathrm{H} 15 \mathrm{NO}$ & 285.1154 & FIU 0681 \\
\hline 2-Fluoropentylindole & $\mathrm{C} 13 \mathrm{H} 16 \mathrm{FN}$ & 205.1267 & FIU 0682 \\
\hline 3-Fluoropentylindole & $\mathrm{C} 13 \mathrm{H} 16 \mathrm{FN}$ & 205.1267 & FIU 0683 \\
\hline 4-Fluoropentylindole & C13H16FN & 205.1267 & FIU 0684 \\
\hline $\begin{array}{l}\text { 5-fluoropentyl-3- } \\
\text { pyridinoylindole }\end{array}$ & C19H19FN2O & 310.1481 & FIU 0685 \\
\hline 5-Fluoropentylindole & $\mathrm{C} 13 \mathrm{H} 16 \mathrm{FN}$ & 205.1267 & FIU 0686 \\
\hline 5-IT & $\mathrm{C} 11 \mathrm{H} 14 \mathrm{~N} 2$ & 174.1157 & FIU 0687 \\
\hline 6-IT & $\mathrm{C} 11 \mathrm{H} 14 \mathrm{~N} 2$ & 174.1157 & FIU 0688 \\
\hline A-834735 & $\mathrm{C} 22 \mathrm{H} 29 \mathrm{NO} 2$ & 339.2198 & FIU 0689 \\
\hline A-834735 degredant & $\mathrm{C} 22 \mathrm{H} 29 \mathrm{NO} 2$ & 339.2198 & FIU 0690 \\
\hline $\begin{array}{l}\text { methyl-1-(5-fluoropentyl)-1H- } \\
\text { indole-3-Carboxylate }\end{array}$ & C15H18FNO2 & 263.1322 & FIU 0691 \\
\hline $\begin{array}{c}\text { methyl-1-(cyclohexylmethyl)- } \\
\text { 1H-indole-3-carboxylate }\end{array}$ & $\mathrm{C} 17 \mathrm{H} 21 \mathrm{NO} 2$ & 271.1572 & FIU 0692 \\
\hline $\begin{array}{l}\text { methyl-1-pentyl-1H-indole-3- } \\
\text { Carboxylate } \\
\end{array}$ & C15H19NO2 & 245.1416 & FIU 0693 \\
\hline ADBICA & $\mathrm{C} 20 \mathrm{H} 29 \mathrm{~N} 3 \mathrm{O} 2$ & 343.2260 & FIU 0694 \\
\hline $\begin{array}{l}\text { ADBICA N-(4-hydroxypentyl) } \\
\text { metabolite }\end{array}$ & $\mathrm{C} 20 \mathrm{H} 29 \mathrm{~N} 3 \mathrm{O} 3$ & 359.2209 & FIU 0695 \\
\hline $\begin{array}{l}\text { ADBICA N-(5-hydroxypentyl) } \\
\text { metabolite }\end{array}$ & $\mathrm{C} 20 \mathrm{H} 29 \mathrm{~N} 3 \mathrm{O} 3$ & 359.2209 & FIU 0696 \\
\hline $\begin{array}{l}\text { ADBICA N-pentanoic acid } \\
\text { metabolite }\end{array}$ & $\mathrm{C} 20 \mathrm{H} 27 \mathrm{~N} 3 \mathrm{O} 4$ & 373.2002 & FIU 0697 \\
\hline $\begin{array}{l}\text { AM694 N-(5-hydroxypentyl) } \\
\text { metabolite }\end{array}$ & C20H20INO2 & 433.0539 & FIU 0698 \\
\hline $\begin{array}{c}\text { AM694 N-pentanoic acid } \\
\text { metabolite }\end{array}$ & C20H18INO3 & 447.0331 & FIU 0699 \\
\hline Harmine & $\mathrm{C} 13 \mathrm{H} 12 \mathrm{~N} 2 \mathrm{O}$ & 212.0950 & FIU 0700 \\
\hline tetrahydro-Harmine & $\mathrm{C} 13 \mathrm{H} 16 \mathrm{~N} 2 \mathrm{O}$ & 216.1263 & FIU 0701 \\
\hline 5-chloro AB-PINACA & $\mathrm{C} 18 \mathrm{H} 25 \mathrm{ClN} 4 \mathrm{O} 2$ & 364.1666 & FIU 0702 \\
\hline 5-fluoro ABICA & $\mathrm{C} 19 \mathrm{H} 26 \mathrm{FN} 3 \mathrm{O} 2$ & 347.2009 & FIU 0703 \\
\hline 5-fluoro AB-PINACA & $\mathrm{C} 18 \mathrm{H} 25 \mathrm{FN} 4 \mathrm{O} 2$ & 348.1962 & FIU 0704 \\
\hline
\end{tabular}




\begin{tabular}{|c|c|c|c|}
\hline $\begin{array}{l}\text { 5-fluoro AB-PINACA N-(4- } \\
\text { hydroxypentyl) metabolite }\end{array}$ & $\mathrm{C} 18 \mathrm{H} 25 \mathrm{FN} 4 \mathrm{O} 3$ & 364.1911 & FIU 0705 \\
\hline 5-fluoro ADBICA & $\mathrm{C} 20 \mathrm{H} 28 \mathrm{FN} 3 \mathrm{O} 2$ & 361.2166 & FIU 0706 \\
\hline 5-fluoro ADB-PINACA & C19H27FN4O2 & 362.2118 & FIU 0707 \\
\hline 5-fluoro AMB & $\mathrm{C} 19 \mathrm{H} 26 \mathrm{FN} 3 \mathrm{O} 3$ & 363.1958 & FIU 0708 \\
\hline 5-fluoro MN-18 & $\mathrm{C} 23 \mathrm{H} 22 \mathrm{FN} 3 \mathrm{O}$ & 375.1747 & FIU 0709 \\
\hline $\begin{array}{c}\text { 5-fluoro-AKB48 N-(4- } \\
\text { hydroxypentyl) metabolite }\end{array}$ & $\mathrm{C} 23 \mathrm{H} 30 \mathrm{FN} 3 \mathrm{O} 2$ & 399.2322 & FIU 0710 \\
\hline 5-fluoro-THJ & $\mathrm{C} 22 \mathrm{H} 21 \mathrm{FN} 4 \mathrm{O}$ & 376.1699 & FIU 0711 \\
\hline AB-005 & $\mathrm{C} 23 \mathrm{H} 32 \mathrm{~N} 2 \mathrm{O}$ & 352.2515 & FIU 0712 \\
\hline AB-005 azepane isomer & $\mathrm{C} 23 \mathrm{H} 32 \mathrm{~N} 2 \mathrm{O}$ & 352.2515 & FIU 0713 \\
\hline AB-CHMINACA & $\mathrm{C} 20 \mathrm{H} 28 \mathrm{~N} 4 \mathrm{O} 2$ & 356.2212 & FIU 0714 \\
\hline AB-FUBINACA & $\mathrm{C} 20 \mathrm{H} 21 \mathrm{FN} 4 \mathrm{O} 2$ & 368.1649 & FIU 0715 \\
\hline $\begin{array}{l}\text { AB-FUBINACA 2- } \\
\text { fluorobenzyl isomer }\end{array}$ & $\mathrm{C} 20 \mathrm{H} 21 \mathrm{FN} 4 \mathrm{O} 2$ & 368.1649 & FIU 0716 \\
\hline $\begin{array}{l}\text { AB-FUBINACA 3- } \\
\text { fluorobenzyl isomer }\end{array}$ & $\mathrm{C} 20 \mathrm{H} 21 \mathrm{FN} 4 \mathrm{O} 2$ & 368.1649 & FIU 0717 \\
\hline AB-FUBINACA isomer 1 & $\mathrm{C} 20 \mathrm{H} 21 \mathrm{FN} 4 \mathrm{O} 2$ & 368.1649 & FIU 0718 \\
\hline AB-FUBINACA isomer 2 & $\mathrm{C} 20 \mathrm{H} 21 \mathrm{FN} 4 \mathrm{O} 2$ & 368.1649 & FIU 0719 \\
\hline AB-FUBINACA isomer 5 & $\mathrm{C} 20 \mathrm{H} 21 \mathrm{FN} 4 \mathrm{O} 2$ & 368.1649 & FIU 0720 \\
\hline AB-PINACA & $\mathrm{C} 18 \mathrm{H} 26 \mathrm{~N} 4 \mathrm{O} 2$ & 330.2056 & FIU 0721 \\
\hline $\begin{array}{l}\text { AB-PINACA N-(2- } \\
\text { fluoropentyl) isomer }\end{array}$ & $\mathrm{C} 18 \mathrm{H} 25 \mathrm{FN} 4 \mathrm{O} 2$ & 348.1963 & FIU 0722 \\
\hline $\begin{array}{l}\text { AB-PINACA N-(4- } \\
\text { fluoropentyl) isomer }\end{array}$ & $\mathrm{C} 18 \mathrm{H} 25 \mathrm{FN} 4 \mathrm{O} 2$ & 348.1963 & FIU 0723 \\
\hline $\begin{array}{c}\text { AB-PINACA N-(4- } \\
\text { hydroxypentyl) metabolite }\end{array}$ & $\mathrm{C} 18 \mathrm{H} 26 \mathrm{~N} 4 \mathrm{O} 3$ & 346.2005 & FIU 0724 \\
\hline $\begin{array}{c}\text { AB-PINACA N-(5- } \\
\text { hydroxypentyl) metabolite }\end{array}$ & $\mathrm{C} 18 \mathrm{H} 26 \mathrm{~N} 4 \mathrm{O} 3$ & 346.2005 & FIU 0725 \\
\hline $\begin{array}{l}\text { AB-PINACA pentanoic acid } \\
\text { metabolite }\end{array}$ & $\mathrm{C} 18 \mathrm{H} 24 \mathrm{~N} 4 \mathrm{O} 4$ & 360.1798 & FIU 0726 \\
\hline $\begin{array}{l}\text { AKB48 N-(5-fluoropentyl) } \\
\text { analog }\end{array}$ & $\mathrm{C} 23 \mathrm{H} 30 \mathrm{FN} 3 \mathrm{O}$ & 383.2373 & FIU 0727 \\
\hline MN-18 & $\mathrm{C} 23 \mathrm{H} 23 \mathrm{~N} 3 \mathrm{O}$ & 357.1841 & FIU 0728 \\
\hline THJ & $\mathrm{C} 22 \mathrm{H} 22 \mathrm{~N} 4 \mathrm{O}$ & 358.1794 & FIU 0729 \\
\hline THJ 018 & $\mathrm{C} 23 \mathrm{H} 22 \mathrm{~N} 2 \mathrm{O}$ & 342.1732 & FIU 0730 \\
\hline THJ 2201 & $\mathrm{C} 23 \mathrm{H} 21 \mathrm{FN} 2 \mathrm{O}$ & 360.1638 & FIU 0731 \\
\hline ADB-FUBINACA & $\mathrm{C} 21 \mathrm{H} 23 \mathrm{FN} 4 \mathrm{O} 2$ & 382.1805 & FIU 0732 \\
\hline ADB-PINACA & $\mathrm{C} 19 \mathrm{H} 28 \mathrm{~N} 4 \mathrm{O} 2$ & 344.2212 & FIU 0733 \\
\hline ADB-PINACA isomer 1 & $\mathrm{C} 19 \mathrm{H} 28 \mathrm{~N} 4 \mathrm{O} 2$ & 344.2212 & FIU 0734 \\
\hline ADB-PINACA isomer 2 & $\mathrm{C} 19 \mathrm{H} 28 \mathrm{~N} 4 \mathrm{O} 2$ & 344.2212 & FIU 0735 \\
\hline ADB-PINACA isomer 3 & $\mathrm{C} 19 \mathrm{H} 28 \mathrm{~N} 4 \mathrm{O} 2$ & 344.2212 & FIU 0736 \\
\hline
\end{tabular}




\begin{tabular}{|c|c|c|c|}
\hline $\begin{array}{c}\text { ADB-PINACA N-(4- } \\
\text { hydroxypentyl) metabolite }\end{array}$ & C19H28N4O3 & 360.2161 & FIU 0737 \\
\hline $\begin{array}{l}\text { ADB-PINACA N-(5- } \\
\text { hydroxypentyl) metabolite }\end{array}$ & C19H28N4O3 & 360.2161 & FIU 0738 \\
\hline $\begin{array}{l}\text { ADB-PINACA pentanoic acid } \\
\text { metabolite }\end{array}$ & $\mathrm{C} 19 \mathrm{H} 26 \mathrm{~N} 4 \mathrm{O} 4$ & 374.1954 & FIU 0739 \\
\hline $\begin{array}{l}\text { AKB48 N-(4-fluorobenzyl) } \\
\text { analog }\end{array}$ & $\mathrm{C} 25 \mathrm{H} 26 \mathrm{FN} 3 \mathrm{O}$ & 403.2060 & FIU 0740 \\
\hline $\begin{array}{l}\text { AKB48 N-(4-hydroxypentyl) } \\
\text { metabolite }\end{array}$ & $\mathrm{C} 23 \mathrm{H} 31 \mathrm{~N} 3 \mathrm{O} 2$ & 381.2416 & FIU 0741 \\
\hline $\begin{array}{c}\text { AKB48 N-(5-hydroxypentyl) } \\
\text { metabolite }\end{array}$ & $\mathrm{C} 23 \mathrm{H} 31 \mathrm{~N} 3 \mathrm{O} 2$ & 381.2416 & FIU 0742 \\
\hline $\begin{array}{c}\text { AKB48 N-pentanoic acid } \\
\text { metabolite }\end{array}$ & $\mathrm{C} 23 \mathrm{H} 29 \mathrm{~N} 3 \mathrm{O} 3$ & 395.2209 & FIU 0743 \\
\hline AMB & $\mathrm{C} 19 \mathrm{H} 27 \mathrm{~N} 3 \mathrm{O} 3$ & 345.2052 & FIU 0744 \\
\hline 4-acetoxy DiPT & $\mathrm{C} 18 \mathrm{H} 26 \mathrm{~N} 2 \mathrm{O} 2$ & 302.1994 & FIU 0745 \\
\hline 4-acetoxy DMT & $\mathrm{C} 14 \mathrm{H} 18 \mathrm{~N} 2 \mathrm{O} 2$ & 246.1368 & FIU 0746 \\
\hline 4-hydroxy DET & C14H20N2O & 232.1576 & FIU 0747 \\
\hline 4-hydroxy DiPT & $\mathrm{C} 16 \mathrm{H} 24 \mathrm{~N} 2 \mathrm{O}$ & 260.1889 & FIU 0748 \\
\hline 4-hydroxy MET & $\mathrm{C} 13 \mathrm{H} 18 \mathrm{~N} 2 \mathrm{O}$ & 218.1419 & FIU 0749 \\
\hline 4-hydroxy MiPT & C14H20N2O & 232.1576 & FIU 0750 \\
\hline 4-methyl- $\alpha$-Ethyltryptamine & C13H18N2 & 202.1470 & FIU 0751 \\
\hline 5-methoxy- $\alpha$-Ethyltryptamine & $\mathrm{C} 13 \mathrm{H} 18 \mathrm{~N} 2 \mathrm{O}$ & 218.1419 & FIU 0752 \\
\hline DiPT & $\mathrm{C} 16 \mathrm{H} 24 \mathrm{~N} 2$ & 244.1939 & FIU 0753 \\
\hline DOET & $\mathrm{C} 13 \mathrm{H} 21 \mathrm{NO} 2$ & 223.1572 & FIU 0754 \\
\hline DPT & $\mathrm{C} 16 \mathrm{H} 24 \mathrm{~N} 2$ & 244.1939 & FIU 0755 \\
\hline N-Methyltryptamine & $\mathrm{C} 11 \mathrm{H} 14 \mathrm{~N} 2$ & 174.1157 & FIU 0756 \\
\hline $\mathrm{AMT}$ & C5H10N2S & 130.0565 & FIU 0757 \\
\hline Methylphenidate & C14H19NO2 & 233.1416 & FIU 0758 \\
\hline MMAI & $\mathrm{C} 11 \mathrm{H} 15 \mathrm{NO}$ & 177.1154 & FIU 0759 \\
\hline Etaqualone & C17H16N2O & 264.1263 & FIU 0760 \\
\hline Hydroxy Bupropion & C13H18CINO2 & 255.1026 & FIU 0761 \\
\hline Lagochiline & $\mathrm{C} 20 \mathrm{H} 36 \mathrm{O} 5$ & 356.2563 & FIU 0762 \\
\hline Levamisole & C11H12N2S & 204.0721 & FIU 0763 \\
\hline Loperamide & $\mathrm{C} 29 \mathrm{H} 33 \mathrm{ClN} 2 \mathrm{O} 2$ & 476.2231 & FIU 0764 \\
\hline $\begin{array}{l}\text { N-Phenylacetyl-L- } \\
\text { prolylglycine ethyl ester }\end{array}$ & $\mathrm{C} 17 \mathrm{H} 22 \mathrm{~N} 2 \mathrm{O} 4$ & 318.1580 & FIU 0765 \\
\hline Phenylpiracetam & $\mathrm{C} 12 \mathrm{H} 14 \mathrm{~N} 2 \mathrm{O} 2$ & 218.1055 & FIU 0766 \\
\hline PRE-084 & $\mathrm{C} 19 \mathrm{H} 27 \mathrm{NO} 3$ & 317.1991 & FIU 0767 \\
\hline Sildenafil & $\mathrm{C} 22 \mathrm{H} 30 \mathrm{~N} 6 \mathrm{O} 4 \mathrm{~S}$ & 474.2049 & FIU 0768 \\
\hline Sildenafil Citrate & C22H30N6O4S & 474.2049 & FIU 0769 \\
\hline Thiosildenafil & $\mathrm{C} 22 \mathrm{H} 30 \mathrm{~N} 6 \mathrm{O} 3 \mathrm{~S} 2$ & 490.1821 & FIU 0770 \\
\hline
\end{tabular}




\begin{tabular}{|c|c|c|c|}
\hline Acetildenafil & $\mathrm{C} 25 \mathrm{H} 34 \mathrm{~N} 6 \mathrm{O} 3$ & 466.2692 & FIU 0771 \\
\hline Benzydamine & $\mathrm{C} 19 \mathrm{H} 23 \mathrm{~N} 3 \mathrm{O}$ & 309.1841 & FIU 0772 \\
\hline Boldenone Cypionate & $\mathrm{C} 27 \mathrm{H} 38 \mathrm{O} 3$ & 410.2821 & FIU 0773 \\
\hline Caffeine & $\mathrm{C} 8 \mathrm{H} 10 \mathrm{~N} 4 \mathrm{O} 2$ & 194.0804 & FIU 0774 \\
\hline Carisoprodol & $\mathrm{C} 12 \mathrm{H} 24 \mathrm{~N} 2 \mathrm{O} 4$ & 260.1736 & FIU 0775 \\
\hline Cl-2201 & $\mathrm{C} 24 \mathrm{H} 21 \mathrm{ClFNO}$ & 393.1296 & FIU 0776 \\
\hline Clencyclohexerol & $\mathrm{C} 14 \mathrm{H} 20 \mathrm{Cl} 2 \mathrm{~N} 2 \mathrm{O} 2$ & 318.0902 & FIU 0777 \\
\hline
\end{tabular}


Appendix 2. Compounds included in the internal standard database with their unique in-house identifying number.

\begin{tabular}{|c|c|c|c|}
\hline Compound Name & Formula & $\begin{array}{l}\text { Accurate } \\
\text { Mass }\end{array}$ & FIU Number \\
\hline JWH 007-d9 & $\mathrm{C} 22 \mathrm{H} 16 \mathrm{D} 9 \mathrm{NO}$ & 328.2501 & FIU 0778 \\
\hline JWH 015-d 7 & $\mathrm{C} 23 \mathrm{H} 14 \mathrm{D} 7 \mathrm{NO}$ & 334.2063 & FIU 0779 \\
\hline JWH 018-d9 & C24H14D9NO & 350.2345 & FIU 0780 \\
\hline $\begin{array}{l}\text { JWH } 018 \text { 2-hydroxyindole } \\
\text { metabolite-d9 }\end{array}$ & C24H14D9NO2 & 366.2294 & FIU 0781 \\
\hline $\begin{array}{l}\text { JWH } 018 \text { 4-hydroxyindole } \\
\text { metabolite-d9 }\end{array}$ & C24H14D9NO2 & 366.2294 & FIU 0782 \\
\hline $\begin{array}{l}\text { JWH } 018 \text { 5-hydroxyindole } \\
\text { metabolite-d } 9\end{array}$ & $\mathrm{C} 24 \mathrm{H} 14 \mathrm{D} 9 \mathrm{NO} 2$ & 366.2294 & FIU 0783 \\
\hline $\begin{array}{l}\text { JWH } 018 \text { 6-hydroxyindole } \\
\text { metabolite-d9 }\end{array}$ & C24H14D9NO2 & 366.2294 & FIU 0784 \\
\hline $\begin{array}{l}\text { JWH } 018 \text { 7-hydroxyindole } \\
\text { metabolite-d9 }\end{array}$ & $\mathrm{C} 24 \mathrm{H} 14 \mathrm{D} 9 \mathrm{NO} 2$ & 366.2294 & FIU 0785 \\
\hline $\begin{array}{l}( \pm)-J W H ~ 018 \mathrm{~N}-(4- \\
\text { hydroxypentyl) } \\
\text { metabolite-d } 5\end{array}$ & $\mathrm{C} 24 \mathrm{H} 18 \mathrm{D} 5 \mathrm{NO} 2$ & 362.2043 & FIU 0786 \\
\hline $\begin{array}{l}\text { JWH } 018 \mathrm{~N}-(5- \\
\text { hydroxypentyl }) \\
\text { metabolite- } \mathrm{d}_{5}\end{array}$ & $\mathrm{C} 24 \mathrm{H} 18 \mathrm{D} 5 \mathrm{NO} 2$ & 362.2043 & FIU 0787 \\
\hline $\begin{array}{l}\text { JWH } 018 \mathrm{~N}-(5- \\
\text { hydroxypentyl) } \beta \text {-D- } \\
\text { Glucuronide-d } 5\end{array}$ & $\mathrm{C} 30 \mathrm{H} 26 \mathrm{D} 5 \mathrm{NO} 8$ & 538.2364 & FIU 0788 \\
\hline $\begin{array}{l}\text { JWH } 018 \text { N-pentanoic } \\
\text { acid metabolite-d } \mathrm{d}_{5}\end{array}$ & $\mathrm{C} 24 \mathrm{H} 16 \mathrm{D} 5 \mathrm{NO} 3$ & 376.1865 & FIU 0789 \\
\hline $\begin{array}{l}\text { JWH } 018 \text { N-pentanoic } \\
\text { acid metabolite-d } \mathrm{d}_{4}\end{array}$ & C24H17D4NO3 & 375.1773 & FIU 0790 \\
\hline $\begin{array}{l}\text { JWH } 019 \text { N-(5- } \\
\text { hydroxyhexyl) metabolite- } \\
d_{5}\end{array}$ & $\mathrm{C} 25 \mathrm{H} 20 \mathrm{D} 5 \mathrm{NO} 2$ & 376.2199 & FIU 0791 \\
\hline JWH 073-d 7 & $\mathrm{C} 23 \mathrm{H} 14 \mathrm{D} 7 \mathrm{NO}$ & 334.2063 & FIU 0792 \\
\hline $\begin{array}{l}( \pm)-J W H \text { 073 N-(3- } \\
\text { hydroxybutyl)metabolite- } \\
d_{5}\end{array}$ & $\mathrm{C} 23 \mathrm{H} 16 \mathrm{D} 5 \mathrm{NO} 2$ & 348.1886 & FIU 0793 \\
\hline $\begin{array}{l}\text { JWH } 073 \mathrm{~N}-(4- \\
\text { hydroxybutyl) metabolite- } \\
\mathrm{d}_{5}\end{array}$ & $\mathrm{C} 23 \mathrm{H} 16 \mathrm{D} 5 \mathrm{NO} 2$ & 348.1886 & FIU 0794 \\
\hline $\begin{array}{l}\text { JWH } 073 \text { 2-hydroxyindole } \\
\text { metabolite-d } 7\end{array}$ & $\mathrm{C} 23 \mathrm{H} 14 \mathrm{D} 7 \mathrm{NO} 2$ & 350.2012 & FIU 0795 \\
\hline $\begin{array}{l}\text { JWH } 073 \text { 4-hydroxyindole } \\
\text { metabolite-d } 7\end{array}$ & $\mathrm{C} 23 \mathrm{H} 14 \mathrm{D} 7 \mathrm{NO} 2$ & 350.2012 & FIU 0796 \\
\hline
\end{tabular}




\begin{tabular}{|c|c|c|c|}
\hline $\begin{array}{l}\text { JWH } 073 \text { 5-hydroxyindole } \\
\text { metabolite- } \mathrm{d}_{7}\end{array}$ & $\mathrm{C} 23 \mathrm{H} 14 \mathrm{D} 7 \mathrm{NO} 2$ & 350.2012 & FIU 0797 \\
\hline $\begin{array}{l}\text { JWH } 073 \text { 6-hydroxyindole } \\
\text { metabolite-d } 7\end{array}$ & $\mathrm{C} 23 \mathrm{H} 14 \mathrm{D} 7 \mathrm{NO} 2$ & 350.2012 & FIU 0798 \\
\hline $\begin{array}{l}\text { JWH } 073 \text { 7-hydroxyindole } \\
\text { metabolite-d7 }\end{array}$ & $\mathrm{C} 23 \mathrm{H} 14 \mathrm{D} 7 \mathrm{NO} 2$ & 350.2012 & FIU 0799 \\
\hline $\begin{array}{l}\text { JWH } 073 \text { N-butanoic acid } \\
\text { metabolite- } \mathrm{d}_{5}\end{array}$ & C23H14D5NO3 & 362.1679 & FIU 0800 \\
\hline JWH 081-d9 & C25H16D9NO2 & 380.2450 & FIU 0801 \\
\hline $\begin{array}{l}\text { JWH } 081 \mathrm{~N}-(4- \\
\text { hydroxypentyl) } \\
\text { metabolite-d } 5\end{array}$ & $\mathrm{C} 25 \mathrm{H} 20 \mathrm{D} 5 \mathrm{NO} 3$ & 392.2148 & FIU 0802 \\
\hline $\begin{array}{l}\text { JWH } 081 \mathrm{~N}-(5- \\
\text { hydroxypentyl) } \\
\text { metabolite-d } \mathrm{d}_{5}\end{array}$ & $\mathrm{C} 25 \mathrm{H} 20 \mathrm{D} 5 \mathrm{NO} 3$ & 392.2148 & FIU 0803 \\
\hline $\begin{array}{l}\text { JWH } 081 \text { N-pentanoic } \\
\text { acid metabolite- } \mathrm{d}_{5}\end{array}$ & $\mathrm{C} 25 \mathrm{H} 18 \mathrm{D} 5 \mathrm{NO} 4$ & 406.1941 & FIU 0804 \\
\hline JWH 122-d9 & C25H16D9NO & 364.2501 & FIU 0805 \\
\hline $\begin{array}{l}\text { JWH } 122 \mathrm{~N}-(5- \\
\text { hydroxypentyl) } \\
\text { metabolite- } \mathrm{d}_{5}\end{array}$ & $\mathrm{C} 25 \mathrm{H} 20 \mathrm{D} 5 \mathrm{NO} 2$ & 376.2199 & FIU 0806 \\
\hline JWH $200-d_{5}$ & C25H19D5N2O2 & 389.2152 & FIU 0807 \\
\hline $\begin{array}{l}\text { JWH } 203 \mathrm{~N}-(4- \\
\text { hydroxypentyl) } \\
\text { metabolite-d } 5\end{array}$ & C21H17D5CINO2 & 360.1653 & FIU 0808 \\
\hline $\begin{array}{l}\text { JWH } 203 \mathrm{~N}-(5- \\
\text { hydroxypentyl) } \\
\text { metabolite- } \mathrm{d}_{5}\end{array}$ & C21H17D5ClNO2 & 360.1653 & FIU 0809 \\
\hline $\begin{array}{l}\text { JWH } 203 \text { N-pentanoic } \\
\text { acid metabolite- } \mathrm{d}_{5}\end{array}$ & C21H15D5C1NO3 & 360.1653 & FIU 0810 \\
\hline JWH $210-d_{9}$ & C26H10D9NO & 370.2032 & FIU 0811 \\
\hline JWH $250-\mathrm{d}_{5}$ & $\mathrm{C} 22 \mathrm{H} 20 \mathrm{D} 5 \mathrm{NO} 2$ & 340.2199 & FIU 0812 \\
\hline $\begin{array}{l}\text { JWH } 250 \mathrm{~N}-(4- \\
\text { hydroxypentyl) } \\
\text { metabolite-d } 5\end{array}$ & $\mathrm{C} 22 \mathrm{H} 20 \mathrm{D} 5 \mathrm{NO} 3$ & 356.2148 & FIU 0813 \\
\hline $\begin{array}{l}\text { JWH } 250 \mathrm{~N}-(5- \\
\text { hydroxypentyl }) \\
\text { metabolite-d } 5\end{array}$ & $\mathrm{C} 22 \mathrm{H} 20 \mathrm{D} 5 \mathrm{NO} 3$ & 356.2148 & FIU 0814 \\
\hline JWH 398-d9 & C24H13D9ClNO & 384.1955 & FIU 0815 \\
\hline $\begin{array}{l}( \pm)-J W H 398 \mathrm{~N}-(4- \\
\text { hydroxypentyl) } \\
\text { metabolite-d } \mathrm{d}_{5}\end{array}$ & $\mathrm{C} 24 \mathrm{H} 17 \mathrm{ClD} 5 \mathrm{NO} 2$ & 396.1653 & FIU 0816 \\
\hline
\end{tabular}




\begin{tabular}{|c|c|c|c|}
\hline $\begin{array}{l}\text { JWH } 398 \mathrm{~N}-(5- \\
\text { hydroxypentyl) metabolite } \\
d_{5}\end{array}$ & C24H17ClD5NO2 & 396.1653 & FIU 0817 \\
\hline $\begin{array}{l}\text { JWH } 398 \text { N-pentanoic } \\
\text { acid metabolite-d } \mathrm{d}_{5}\end{array}$ & $\mathrm{C} 24 \mathrm{H} 15 \mathrm{ClD} 5 \mathrm{NO} 3$ & 410.1446 & FIU 0818 \\
\hline $\begin{array}{l}\text { (-)-11-nor-9-carboxy- } \Delta 9- \\
\text { THC-d }_{3}\end{array}$ & $\mathrm{C} 21 \mathrm{H} 25 \mathrm{D} 3 \mathrm{O} 4$ & 347.2176 & FIU 0819 \\
\hline$( \pm)-\mathrm{CP} 47,497-\mathrm{d}_{11}$ & $\mathrm{C} 21 \mathrm{H} 23 \mathrm{D} 11 \mathrm{O} 2$ & 329.3249 & FIU 0820 \\
\hline $\begin{array}{l}( \pm)-C P 47,497-C 8- \\
\text { homolog-d } 7\end{array}$ & $\mathrm{C} 22 \mathrm{H} 29 \mathrm{D} 7 \mathrm{O} 2$ & 339.3155 & FIU 0821 \\
\hline$( \pm)-\mathrm{CP} 55,940-\mathrm{d}_{11}$ & C24H29D11O3 & 387.3668 & FIU 0822 \\
\hline $\begin{array}{l}\text { AM694 N-pentanoic acid } \\
\text { metabolite-d }_{5}\end{array}$ & C20H13D5INO3 & 452.0645 & FIU 0823 \\
\hline AM2201-d 5 & C24H17D5FNO & 364.1999 & FIU 0824 \\
\hline $\begin{array}{l}\text { AM2201 N-(4- } \\
\text { hydroxypentyl) } \\
\text { metabolite-d } 5\end{array}$ & C24H17D5FNO2 & 380.1948 & FIU 0825 \\
\hline MAM2201-d 5 & C25H19D5FNO & 378.2156 & FIU 0826 \\
\hline $\begin{array}{l}\text { MAM2201 N-pentanoic } \\
\text { acid metabolite-d } \text { d }_{5}\end{array}$ & $\mathrm{C} 25 \mathrm{H} 18 \mathrm{D} 5 \mathrm{NO} 3$ & 390.1992 & FIU 0827 \\
\hline PB-22-d9 & C23H13D9N2O2 & 367.2246 & FIU 0828 \\
\hline UR-144-d 5 & $\mathrm{C} 21 \mathrm{H} 24 \mathrm{D} 5 \mathrm{NO}$ & 316.2563 & FIU 0829 \\
\hline $\begin{array}{l}\text { UR-144 N-(4- } \\
\text { hydroxypentyl) } \\
\text { metabolite-d5 }\end{array}$ & $\mathrm{C} 21 \mathrm{H} 24 \mathrm{D} 5 \mathrm{NO} 2$ & 332.2512 & FIU 0830 \\
\hline $\begin{array}{l}\text { UR-144 N-(5- } \\
\text { hydroxypentyl) } \\
\text { metabolite-d } 5\end{array}$ & $\mathrm{C} 21 \mathrm{H} 24 \mathrm{D} 5 \mathrm{NO} 2$ & 332.2512 & FIU 0831 \\
\hline $\begin{array}{l}\text { UR-144 N-pentanoic acid } \\
\text { metabolite-d }{ }_{5}\end{array}$ & $\mathrm{C} 21 \mathrm{H} 22 \mathrm{D} 5 \mathrm{NO} 3$ & 346.2305 & FIU 0832 \\
\hline XLR11-d 5 & $\mathrm{C} 21 \mathrm{H} 23 \mathrm{D} 5 \mathrm{FNO}$ & 334.2469 & FIU 0833 \\
\hline $\begin{array}{l}\text { XLR11 N-(4- } \\
\text { hydroxypentyl) } \\
\text { metabolite-d } 5\end{array}$ & C21H23D5FNO2 & 350.2418 & FIU 0834 \\
\hline RCS-4-d 9 & C21H14D9NO2 & 330.2294 & FIU 0835 \\
\hline $\begin{array}{l}\text { RCS-4 N-(4- } \\
\text { hydroxypentyl) } \\
\text { metabolite-d } 5\end{array}$ & $\mathrm{C} 21 \mathrm{H} 18 \mathrm{D} 5 \mathrm{NO} 3$ & 342.1992 & FIU 0836 \\
\hline $\begin{array}{l}\text { RCS-4 N-(5- } \\
\text { hydroxypentyl) } \\
\text { metabolite-d } 5\end{array}$ & $\mathrm{C} 21 \mathrm{H} 18 \mathrm{D} 5 \mathrm{NO} 3$ & 342.1992 & FIU 0837 \\
\hline $\begin{array}{l}\text { RCS-4 N-pentanoic acid } \\
\text { metabolite-d } \mathrm{d}_{5}\end{array}$ & $\mathrm{C} 21 \mathrm{H} 16 \mathrm{D} 5 \mathrm{NO} 4$ & 356.1784 & FIU 0838 \\
\hline
\end{tabular}




\begin{tabular}{|c|c|c|c|}
\hline $25 \mathrm{I}-\mathrm{NBOMe}-\mathrm{d}_{3}$ & C18H19D3INO3 & 430.0833 & FIU 0839 \\
\hline Benocyclidine- $\mathrm{d}_{10}$ & C19H15D10NS & 309.2335 & FIU 0840 \\
\hline $\begin{array}{l}\text { 3,4-Methylenedioxy } \\
\text { Pyrovalerone- } \mathrm{d}_{8}\end{array}$ & C16H13D8NO3 & 283.2024 & FIU 0841 \\
\hline AB-PINACA-d, 9 & C18H17D9N4O2 & 339.2621 & FIU 0842 \\
\hline ADB-PINACA-d 9 & C19H19D9N4O2 & 353.2777 & FIU 0843 \\
\hline ADBICA-d 9 & $\mathrm{C} 20 \mathrm{H} 20 \mathrm{D} 9 \mathrm{~N} 3 \mathrm{O} 2$ & 352.2825 & FIU 0844 \\
\hline AB-FUBINACA-d 4 & C20H17D4FN4O2 & 372.1900 & FIU 0845 \\
\hline AKB48-d9 & C23H22D9N3O & 374.3032 & FIU 0846 \\
\hline Acetyl fentanyl-d $\mathrm{d}_{5}$ & $\mathrm{C} 21 \mathrm{H} 22 \mathrm{D} 5 \mathrm{CIN} 2 \mathrm{O}$ & 363.2126 & FIU 0847 \\
\hline Acetyl norfentanyl-d 5 & C13H13D5N2O & 223.1733 & FIU 0848 \\
\hline Norsufentanil- $\mathrm{d}_{3}$ & $\mathrm{C} 16 \mathrm{H} 21 \mathrm{D} 3 \mathrm{~N} 2 \mathrm{O} 2$ & 279.2026 & FIU 0849 \\
\hline Butylone- $\mathrm{d}_{3}$ & $\mathrm{C} 12 \mathrm{H} 12 \mathrm{D} 3 \mathrm{NO} 3$ & 224.1240 & FIU 0850 \\
\hline cis-Tramadol- $\mathrm{d}_{6}$ & C16H19D6NO2 & 269.2262 & FIU 0851 \\
\hline Meconin- $\mathrm{d}_{3}$ & C10H7D3O4 & 197.0767 & FIU 0852 \\
\hline
\end{tabular}


Appendix 3. The novel psychoactive substances added to the HRMS library along with ions collected at different collision energies that had relative abundances greater than $10 \%$ in the MS/MS spectra.

\begin{tabular}{|c|c|c|}
\hline Compound Name & $\begin{array}{c}\mathrm{CE} \\
(\mathrm{eV})\end{array}$ & Ion (Relative Abundance \%) \\
\hline \multirow{3}{*}{$( \pm)$-Ethylphenidate } & 10 & $248.16451(100) ; 84.08077(56)$ \\
\hline & 20 & $84.08077(100)$ \\
\hline & 40 & 84.08077 (100); $56.04948(17)$ \\
\hline \multirow{3}{*}{ 2-Bromoamphetamine } & 10 & $\begin{array}{l}168.96474(100) ; 196.99603(57) ; \\
214.02258(21)\end{array}$ \\
\hline & 20 & $168.96474(100)$ \\
\hline & 40 & $\begin{array}{l}90.04640 \text { (100); } 168.96474(87) ; \\
89.03857 \text { (43); } 117.06988(24) ; \\
91.05423(11)\end{array}$ \\
\hline \multirow{3}{*}{ 2-Chloroamphetamine } & 10 & $\begin{array}{l}125.01525(100) ; 153.04655(33) ; \\
170.07310(11)\end{array}$ \\
\hline & 20 & $125.01525(100)$ \\
\hline & 40 & $\begin{array}{l}170.01525(100) ; 89.03857(75) ; \\
98.99960(40) ; 90.04640(30) ; 63.02293 \\
(10)\end{array}$ \\
\hline \multirow{3}{*}{ 2-Methoxy ketamine } & 10 & $\begin{array}{l}203.10666 \text { (100); } 175.11174(79) ; \\
234.14885(33)\end{array}$ \\
\hline & 20 & $\begin{array}{l}121.06479(100) ; 175.11174(93) ; \\
203.10666(54) ; 67.05423(14)\end{array}$ \\
\hline & 40 & $\begin{array}{l}91.05423(100) ; 121.06479(43) ; \\
67.05423(12)\end{array}$ \\
\hline \multirow[b]{3}{*}{$\begin{array}{c}3,4- \\
\text { Ethylenedioxymethcathinone }\end{array}$} & 10 & $204.10190(100) ; 222.11247(46)$ \\
\hline & 20 & $\begin{array}{l}204.10190(100) ; 189.07843(83) ; \\
\text { 148.07568 (34); } 163.07536(16) ; \\
58.06512(16) ; 133.05222(12)\end{array}$ \\
\hline & 40 & $\begin{array}{l}133.05222(100) ; 91.05423(47) ; \\
148.07568(40) ; 120.08077(20) ; \\
105.05730(20) ; 58.06512(18) ; \\
189.07843(14) ; 77.03857(13) ; \\
65.03857(10)\end{array}$ \\
\hline \multirow{3}{*}{ 3-Bromoamphetamine } & 10 & $\begin{array}{l}196.99603 \text { (100); } 168.96474(96) ; \\
214.02258(13) ; 118.07771(12)\end{array}$ \\
\hline & 20 & $\begin{array}{l}168.96474(100) ; 118.07771(23) ; \\
117.06988(13)\end{array}$ \\
\hline & 40 & $\begin{array}{l}117.06988(100) ; 90.04640(81) ; \\
168.96474(75) ; 89.03857(28) ; \\
91.05423(21) ; 115.05423(14)\end{array}$ \\
\hline \multirow{2}{*}{ 3-Chloroamphetamine } & 10 & $125.01525(100) ; 153.04655(54)$ \\
\hline & 20 & $125.01525(100)$ \\
\hline
\end{tabular}




\begin{tabular}{|c|c|c|}
\hline & 40 & $\begin{array}{l}125.01525(100) ; 89.03857(65) \\
98.99960(34) ; 90.04640(24)\end{array}$ \\
\hline \multirow{3}{*}{ 3-Iodoamphetamine } & 10 & $\begin{array}{l}244.98216(100) ; 216.95087 \text { (97); } \\
262.00873(43)\end{array}$ \\
\hline & 20 & $216.95087(100) ; 118.07771(13)$ \\
\hline & 40 & $\begin{array}{l}90.04640(100) ; 117.06988(74) \\
216.95087(51) ; 89.03857(23)\end{array}$ \\
\hline \multirow[b]{3}{*}{ 3-Methoxyamphetamine } & 10 & $121.06398(100) ; 149.09555(51)$ \\
\hline & 20 & $121.06398(100) ; 91.05381(20)$ \\
\hline & 40 & $\begin{array}{l}91.05383(100) ; 78.04608(73) ; \\
65.03838(57) ; 77.03832(47) ; \\
121.06432(26)\end{array}$ \\
\hline \multirow{3}{*}{ 4-Bromoamphetamine } & 10 & $\begin{array}{l}196.99603(100) ; 168.96474(95) ; \\
214.02258(13) ; 118.07771(12)\end{array}$ \\
\hline & 20 & $\begin{array}{l}168.96474(100) ; 118.07771(23) ; \\
117.06988(13)\end{array}$ \\
\hline & 40 & $\begin{array}{l}\text { 117.06988 (100); } 90.04640(81) ; \\
\text { 168.96474 (75); } 89.03857(28) ; \\
91.05423(21) ; 115.05423(14)\end{array}$ \\
\hline \multirow{3}{*}{ 4-Chloroamphetamine } & 10 & $125.01525(100) ; 153.04655(54)$ \\
\hline & 20 & $125.01525(100)$ \\
\hline & 40 & $\begin{array}{l}125.01525(100) ; 89.03857(65) ; \\
98.99960 \text { (34); } 90.04640 \text { (24) }\end{array}$ \\
\hline \multirow[b]{3}{*}{ 4-Hydroxyamphetamine } & 10 & $107.04914(100) ; 135.08044(96)$ \\
\hline & 20 & $107.04914(100)$ \\
\hline & 40 & $\begin{array}{l}77.03857(100) ; 107.04914(42) ; \\
79.05423 \text { (14); } 51.02293 \text { (12); } 91.05423 \\
\text { (11) }\end{array}$ \\
\hline \multirow{3}{*}{ 4-Methylthioamphetamine } & 10 & $\begin{array}{l}165.07324(100) ; 117.06988(32) ; \\
137.04195(25)\end{array}$ \\
\hline & 20 & $\begin{array}{l}137.04195(100) ; 117.06988(99) ; \\
118.07771(27) ; 115.05423(24) ; \\
165.07324(20) ; 150.04977(20) ; \\
135.02629(10)\end{array}$ \\
\hline & 40 & $\begin{array}{l}117.06988 \text { (100); } 91.05423(73) ; \\
115.05423 \text { (70); } 122.01847(48) ; \\
121.01065 \text { (25); } 135.02629(16) ; \\
137.04195 \text { (16); } 78.04640(16)\end{array}$ \\
\hline \multirow{2}{*}{$\begin{array}{l}\text { 4-Methylthioamphetamine } \\
\text { (hydrochloride) }\end{array}$} & 10 & $\begin{array}{l}165.07324(100) ; 117.06988(32) ; \\
137.04195(24)\end{array}$ \\
\hline & 20 & $\begin{array}{l}117.06988(100) ; 137.04195(97) ; \\
118.07771(26) ; 115.05423(23) ;\end{array}$ \\
\hline
\end{tabular}




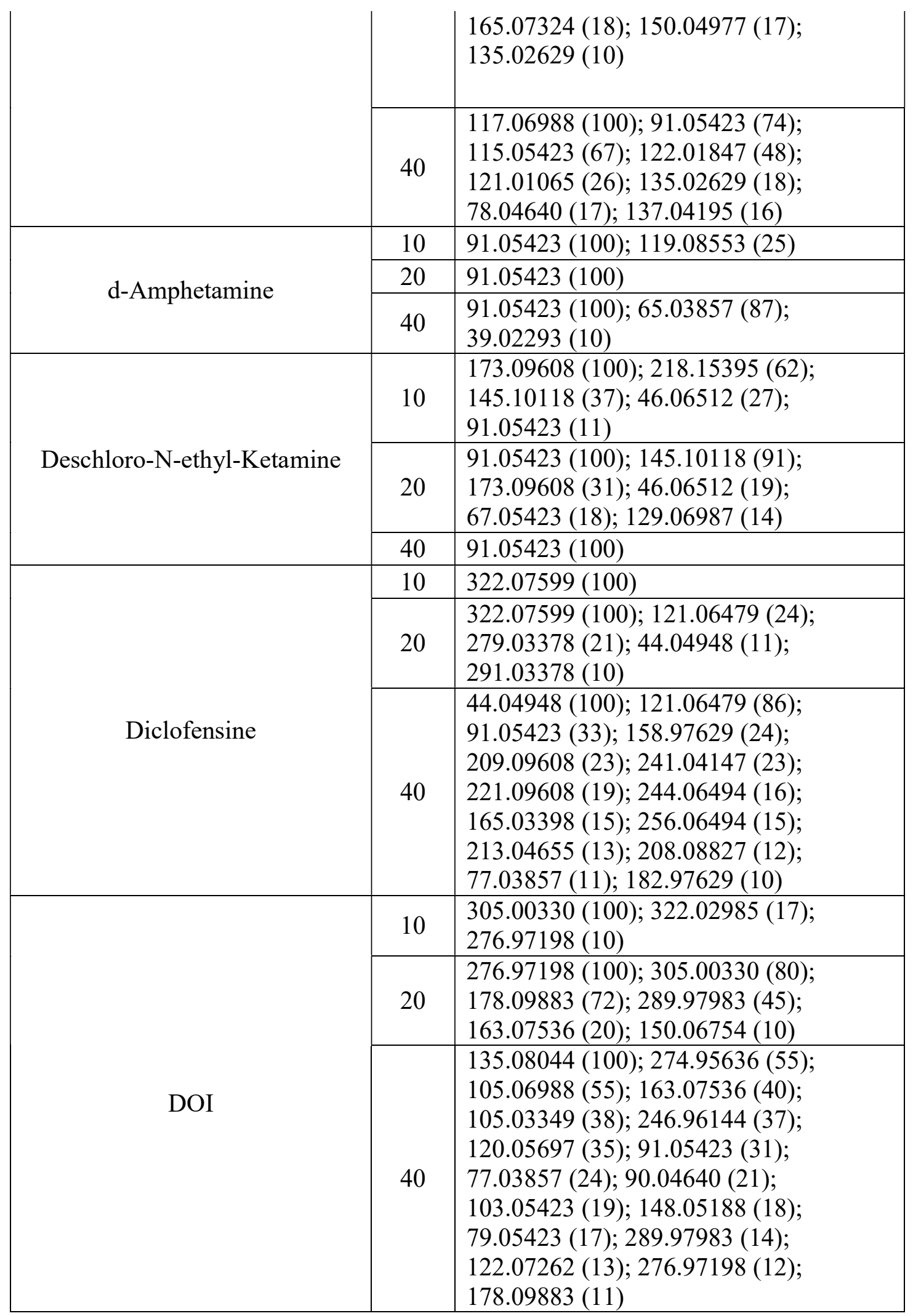




\begin{tabular}{|c|c|c|}
\hline \multirow{3}{*}{ HMA } & 10 & $\begin{array}{l}165.09100(100) ; 137.05971(27) ; \\
133.06479(22) ; 105.06988(13)\end{array}$ \\
\hline & 20 & $\begin{array}{l}\text { 100.06988 (100); } 137.05971(95) ; \\
133.06479(62) ; 165.09100(18) ; \\
79.05423 \text { (17) }\end{array}$ \\
\hline & 40 & $\begin{array}{l}77.03857(100) ; 79.05423(52) ; \\
94.04131(43) ; 103.05423(32) ; \\
105.06988(17) ; 65.03857(17) ; \\
66.04640(16) ; 122.03623(16) ; \\
51.02293(10)\end{array}$ \\
\hline \multirow{3}{*}{ Lisdexamfetamine } & 10 & $\begin{array}{l}264.20703(100) ; 247.18050(38) ; \\
84.08077 \text { (29); } 129.10223(22)\end{array}$ \\
\hline & 20 & $\begin{array}{l}84.08077(100) ; 247.18050(14) \\
119.08553(13) ; 136.11208(10)\end{array}$ \\
\hline & 40 & $\begin{array}{l}84.08077(100) ; 91.05423(37) ; \\
56.04948(16)\end{array}$ \\
\hline \multirow{3}{*}{ Propylhexedrine } & 10 & $\begin{array}{l}156.17468 \text { (100); } 69.06988(43) ; \\
83.08553 \text { (17) }\end{array}$ \\
\hline & 20 & $\begin{array}{l}\text { 69.06988 (100); } 55.05423(46) ; \\
83.08553(35) ; 41.03858(21) ; 57.06988 \\
(11)\end{array}$ \\
\hline & 40 & $\begin{array}{l}55.05423(100) ; 41.03858(90) ; \\
69.06988 \text { (14); } 39.02293(14)\end{array}$ \\
\hline \multirow{3}{*}{ CMP } & 10 & $\begin{array}{l}58.06512(100) ; 79.05423(44) ; \\
93.06988(23) ; 121.10117(12)\end{array}$ \\
\hline & 20 & $\begin{array}{l}79.05423(100) ; 58.06512(63) ; \\
77.03857 \text { (30); } 93.06988(21) ;\end{array}$ \\
\hline & 40 & $\begin{array}{l}77.03857 \text { (100); } 79.05423(19) ; \\
51.02293 \text { (18); } 58.06512(16)\end{array}$ \\
\hline \multirow{3}{*}{ 2,5-DMMA } & 10 & $\begin{array}{l}179.10666(100) ; 151.07536(42) ; \\
210.14885(37) ; 164.08318(13)\end{array}$ \\
\hline & 20 & $\begin{array}{l}\text { 151.07536 (100); } 164.08318(61) ; \\
121.06479(32) ; 179.10666(22) ; \\
149.05971(10) ; 123.08044(10)\end{array}$ \\
\hline & 40 & $\begin{array}{l}91.05423(100) ; 121.06479(73) ; \\
\text { 149.05971 (59); } 77.03857(55) ; \\
78.04640 \text { (23); } 65.03857(16) ; \\
\text { 107.04914 (15); } 93.06988(13) ; \\
\text { 103.05423 (10); }\end{array}$ \\
\hline \multirow{2}{*}{ 3,4-DHMA } & 10 & $\begin{array}{l}151.07536(100), 123.04406(41), \\
182.11756(16)\end{array}$ \\
\hline & 20 & $\begin{array}{l}\text { 123.04406(100), 105.06988(22), } \\
151.07536(14), 133.06479(10)\end{array}$ \\
\hline
\end{tabular}




\begin{tabular}{|c|c|c|}
\hline & 40 & $\begin{array}{l}\text { 77.03858(100), 123.04406(43), } \\
\text { 51.02293(28), 79.05423(28), } \\
\text { 103.05423(16), 105.06988(13), } \\
\text { 105.03349(10), 65.03858(10) }\end{array}$ \\
\hline \multirow[b]{3}{*}{ 4-bromo-2,5-DMMA } & 10 & $\begin{array}{l}257.01717(100), 288.05937(76), \\
228.98587(14)\end{array}$ \\
\hline & 20 & $\begin{array}{l}228.98587(100), 257.01717(68), \\
178.09883(63), 241.99369(31)\end{array}$ \\
\hline & 40 & $\begin{array}{l}\text { 135.08044(100), 226.97022(63), } \\
\text { 198.9753(58), 163.07536(52), } \\
\text { 105.06988(46), 105.03349(34), } \\
91.05423(29), 120.05697(28), \\
\text { 168.96474(23), 77.03858(23), } \\
\text { 178.09883(17), 122.07262(16), } \\
90.0464(15), 228.98587(15), \\
92.06205(15), 241.99369(15), \\
103.05423(14), 148.05188(13), \\
79.05423(11)\end{array}$ \\
\hline \multirow{3}{*}{ para-Methoxymethamphetamine } & 10 & $149.09609(100), 121.06479(38)$ \\
\hline & 20 & $\begin{array}{l}121.06479(100), 149.09609(15), \\
91.05423(11)\end{array}$ \\
\hline & 40 & $\begin{array}{l}\text { 91.05423(100), 121.06479(72), } \\
77.03858(51), 78.0464(47), \\
65.03858(25)\end{array}$ \\
\hline \multirow{3}{*}{ N,N-DMA } & 10 & $\begin{array}{l}\text { 91.05423(100), 164.14338(65), } \\
119.08553(27)\end{array}$ \\
\hline & 20 & $91.05423(100)$ \\
\hline & 40 & $91.05423(100), 65.03858(32)$ \\
\hline \multirow[b]{3}{*}{$2 \mathrm{C}-\mathrm{G}$} & 10 & $\begin{array}{l}178.09883(100), 193.12231(43), \\
210.14886(17)\end{array}$ \\
\hline & 20 & $178.09883(100), 163.07536(41)$ \\
\hline & 40 & $\begin{array}{l}\text { 163.07536(100), 91.05423(76), } \\
\text { 105.06988(40), 135.08044(30), } \\
79.05423(28), 107.08553(23), \\
\text { 115.05423(23), 117.06988(20), } \\
77.03858(14), 133.06479(10)\end{array}$ \\
\hline \multirow[b]{2}{*}{$2 \mathrm{C}-\mathrm{T}$} & 10 & $211.07873(100)$ \\
\hline & 20 & $\begin{array}{l}211.07873(100), 196.05525(60), \\
134.07262(33), 166.04469(28), \\
181.03178(23), 164.08318(22)\end{array}$ \\
\hline
\end{tabular}




\begin{tabular}{|c|c|c|}
\hline & 40 & $\begin{array}{l}91.05423(100), 119.04914(45), \\
121.06479(37), 151.02121(28), \\
181.03178(26), 134.07262(22), \\
123.0263(21), 77.03858(21), \\
147.04406(20), 105.06988(18), \\
103.05423(18), 44.97935(18), \\
79.05423(16), 104.06205(15), \\
136.05188(14), 78.0464(13), \\
135.03148(13), 137.05971(12), \\
165.03686(12), 133.06479(10), \\
149.05971(10)\end{array}$ \\
\hline \multirow[b]{3}{*}{ 2C-T-7 } & 10 & $239.11003(100)$ \\
\hline & 20 & $\begin{array}{l}239.11003(100), 197.06308(34), \\
224.08655(24), 164.08318(17), \\
167.05251(16), 182.0396(15)\end{array}$ \\
\hline & 40 & $\begin{array}{l}\text { 167.01613(100), 91.05423(68), } \\
\text { 134.07262(54), 121.06479(36), } \\
\text { 119.04914(32), 149.05971(28), } \\
\text { 182.0396(27), 152.02904(20), } \\
\text { 125.00556(15), 151.02121(12), } \\
\text { 139.02121(11), 135.03148(11), } \\
77.03858(11), 164.08318(10)\end{array}$ \\
\hline \multirow[b]{3}{*}{ 2C-TFM } & 10 & $\begin{array}{l}\text { 233.07839(100), 250.10494(29), } \\
218.05492(21)\end{array}$ \\
\hline & 20 & $\begin{array}{l}\text { 218.05492(100), 233.07839(54), } \\
203.03144(13)\end{array}$ \\
\hline & 40 & $\begin{array}{l}203.03144(100), 127.03538(34), \\
113.03972(26), 133.0448(24), \\
218.05492(20), 115.05423(13), \\
91.05423(12), 147.04161(11), \\
151.03538(10)\end{array}$ \\
\hline \multirow{3}{*}{ bk-2C-B } & 10 & $\begin{array}{l}\text { 178.06245(100), 177.07843(96), } \\
274.00733(45), 256.98078(40), \\
228.98587(23), 162.05495(17)\end{array}$ \\
\hline & 20 & $\begin{array}{l}\text { 162.05495(100), 178.06245(93), } \\
177.07843(85), 228.98587(37), \\
163.03897(31), 198.9753(24), \\
224.97838(14)\end{array}$ \\
\hline & 40 & $\begin{array}{l}\text { 162.05495(100), 163.03897(50), } \\
\text { 134.06004(33), 119.03657(22), } \\
\text { 77.03858(21), 105.03349(20), } \\
91.05423(16), 181.95999(12), \\
\text { 209.9549(12), 90.0464(12), } \\
147.03148(11)\end{array}$ \\
\hline 3C-B-fly & 10 & \\
\hline
\end{tabular}




\begin{tabular}{|c|c|c|}
\hline & 20 & $\begin{array}{l}281.01717(100), 202.09883(71), \\
252.98587(26)\end{array}$ \\
\hline & 40 & $\begin{array}{l}\text { 187.07536(100), 173.05971(54), } \\
202.09883(36), 159.08044(32), \\
174.06753(28), 145.06479(26), \\
159.04406(18), 131.08553(14), \\
131.04914(13), 252.98587(12), \\
201.09101(10)\end{array}$ \\
\hline \multirow[b]{3}{*}{$3 \mathrm{C}-\mathrm{P}$} & 10 & $195.10157(100), 237.14852(52)$ \\
\hline & 20 & $\begin{array}{l}\text { 195.10157(100), 107.04914(55), } \\
163.07536(47), 167.07027(45), \\
135.08044(23)\end{array}$ \\
\hline & 40 & $\begin{array}{l}\text { 107.04914(100), 91.05423(37), } \\
77.03858(37), 79.05423(35), \\
103.05423(34), 105.06988(22), \\
115.05423(22), 167.07027(16), \\
123.04406(12), 147.04406(10)\end{array}$ \\
\hline \multirow{3}{*}{ N-methyl-2-AI } & 10 & $\begin{array}{l}117.06988(100), 148.11208(42), \\
115.05423(11)\end{array}$ \\
\hline & 20 & $\begin{array}{l}117.06988(100), 115.05423(54), \\
91.05423(23)\end{array}$ \\
\hline & 40 & $\begin{array}{l}\text { 91.05423(100), 115.05423(97), } \\
65.03858(29)\end{array}$ \\
\hline \multirow{3}{*}{ Escaline } & 10 & $209.11722(100), 181.08592(91)$ \\
\hline & 20 & $\begin{array}{l}\text { 181.08592(100), 121.06479(34), } \\
91.05423(24), 166.06245(22), \\
93.06988(20), 149.05971(16), \\
103.05423(13), 77.03858(11)\end{array}$ \\
\hline & 40 & $\begin{array}{l}\text { 91.05423(100), 77.03858(77), } \\
\text { 103.05423(23), 65.03858(20), } \\
\text { 133.02841(20), 78.0464(15), } \\
\text { 123.04406(14), 121.06479(12), } \\
105.03349(10)\end{array}$ \\
\hline \multirow{3}{*}{ Mescaline } & 10 & $195.10157(100), 180.0781(16)$ \\
\hline & 20 & $\begin{array}{l}\text { 195.10157(100), 180.0781(90), } \\
165.05462(85), 164.08318(31), \\
149.05971(21), 133.02841(21), \\
150.06753(17), 133.06479(13), \\
121.06479(12), 137.05971(11), \\
105.06988(10)\end{array}$ \\
\hline & 40 & \\
\hline \multirow{3}{*}{ 2-Amino-1-phenylbutane } & 10 & $91.05423(100), 133.10118(10)$ \\
\hline & 20 & $91.05423(100)$ \\
\hline & 40 & $91.05423(100), 65.03858(81)$ \\
\hline
\end{tabular}




\begin{tabular}{|c|c|c|}
\hline \multirow{3}{*}{ 2-Ethylamino-1-phenylbutane } & 10 & $\begin{array}{l}91.05423(100), 178.15903(39), \\
133.10118(10)\end{array}$ \\
\hline & 20 & $91.05423(100)$ \\
\hline & 40 & $91.05423(100), 65.03858(34)$ \\
\hline \multirow{3}{*}{ 4-CAB } & 10 & $125.01525(100), 167.0622(12)$ \\
\hline & 20 & $125.01525(100)$ \\
\hline & 40 & $\begin{array}{l}\text { 125.01525(100), 89.03858(57), } \\
98.9996(32), 90.0464(22)\end{array}$ \\
\hline \multirow{3}{*}{ Cathine } & 10 & $\begin{array}{l}\text { 134.09643(100), 117.06988(46), } \\
115.05423(16)\end{array}$ \\
\hline & 20 & $\begin{array}{l}115.05423(100), 117.06988(85), \\
91.05423(53), 134.09643(37), \\
56.04948(14)\end{array}$ \\
\hline & 40 & $\begin{array}{l}\text { 91.05423(100), 115.05423(66), } \\
\text { 65.03858(37), 118.06513(16), } \\
77.03858(11)\end{array}$ \\
\hline \multirow[b]{3}{*}{ (R)-(-)-MT-45 } & 10 & $349.26383(100), 181.10118(11)$ \\
\hline & 20 & $\begin{array}{l}\text { 181.10118(100), 169.16993(34), } \\
349.26383(26)\end{array}$ \\
\hline & 40 & $\begin{array}{l}\text { 166.0777(100), 181.10118(79), } \\
\text { 103.05423(67), 165.06988(43), } \\
\text { 179.08553(22), 87.09167(19), } \\
153.06988(11)\end{array}$ \\
\hline \multirow[b]{3}{*}{$(\mathrm{S})-(+)-\mathrm{MT}-45$} & 10 & $349.26383(100), 181.10118(11)$ \\
\hline & 20 & $\begin{array}{l}\text { 181.10118(100), 169.16993(34), } \\
349.26383(25)\end{array}$ \\
\hline & 40 & $\begin{array}{l}\text { 166.0777(100), 181.10118(78), } \\
\text { 103.05423(69), 165.06988(44), } \\
\text { 179.08553(23), 87.09167(20), } \\
153.06988(11)\end{array}$ \\
\hline \multirow{3}{*}{ 2,3-Dichlorophenylpiperazine } & 10 & $231.04503(100), 188.00283(11)$ \\
\hline & 20 & $\begin{array}{l}188.00283(100), 231.04503(81), \\
153.03398(42), 44.04948(15), \\
152.02615(15)\end{array}$ \\
\hline & 40 & $\begin{array}{l}\text { 152.02615(100), 117.0573(59), } \\
\text { 153.03398(43), 118.06513(39), } \\
\text { 44.04948(18), } 91.05423(15), \\
188.00283(14)\end{array}$ \\
\hline \multirow{3}{*}{ MBZP } & 10 & $\begin{array}{l}\text { 191.15428(100), 91.05423(43), } \\
99.09167(15)\end{array}$ \\
\hline & 20 & $\begin{array}{l}\text { 91.05423(100), 99.09167(12), } \\
191.15428(12)\end{array}$ \\
\hline & 40 & $\begin{array}{l}91.05423(100), 65.03858(27), \\
58.06513(10)\end{array}$ \\
\hline
\end{tabular}




\begin{tabular}{|c|c|c|}
\hline \multirow[b]{3}{*}{ MT-45 } & 10 & $349.26383(100), 181.10118(11)$ \\
\hline & 20 & $\begin{array}{l}\text { 181.10118(100), 169.16993(35), } \\
349.26383(25)\end{array}$ \\
\hline & 40 & $\begin{array}{l}\text { 166.0777(100), 181.10118(77), } \\
\text { 103.05423(68), 165.06988(44), } \\
\text { 179.08553(23), 87.09167(19), } \\
153.06988(10)\end{array}$ \\
\hline \multirow{3}{*}{ Mepirapim } & 10 & $214.12264(100), 314.22269(11)$ \\
\hline & 20 & $214.12264(100)$ \\
\hline & 40 & $\begin{array}{l}144.04439(100), 214.12264(35), \\
43.05423(19), 116.04948(12)\end{array}$ \\
\hline \multirow{3}{*}{ 2-Fluoroisocathinone } & 10 & $\begin{array}{l}123.06045(100), 151.05537(76) \\
103.05423(30), 168.08192(23)\end{array}$ \\
\hline & 20 & $\begin{array}{l}103.05423(100), 123.06045(77), \\
77.03858(25)\end{array}$ \\
\hline & 40 & $\begin{array}{l}\text { 77.03858(100), 103.05423(25), } \\
51.02293(17)\end{array}$ \\
\hline \multirow{3}{*}{ 3,4-Dimethylethcathinone } & 10 & $\begin{array}{l}\text { 188.14338(100), 206.15394(59), } \\
159.10425(16), 173.1199(14), \\
160.11208(12)\end{array}$ \\
\hline & 20 & $\begin{array}{l}\text { 188.14338(100), 159.10425(95), } \\
\text { 173.1199(90), 158.09643(62), } \\
\text { 160.11208(45), 133.10118(28), } \\
\text { 145.0886(25), 144.08078(17), } \\
105.06988(10)\end{array}$ \\
\hline & 40 & $\begin{array}{l}\text { 158.09643(100), 144.08078(47), } \\
\text { 105.06988(22), 91.05423(21), } \\
\text { 115.05423(18), 143.07295(18), } \\
145.0886(11), 117.06988(11)\end{array}$ \\
\hline \multirow{3}{*}{$\begin{array}{l}\text { 4-methoxy-N,N- } \\
\text { Dimethylcathinone }\end{array}$} & 10 & $\begin{array}{l}\text { 208.13321(100), 72.08078(47), } \\
163.07536(29), 135.08044(19)\end{array}$ \\
\hline & 20 & $\begin{array}{l}\text { 72.08078(100), 135.08044(76), } \\
163.07536(28), 105.06988(14)\end{array}$ \\
\hline & 40 & $\begin{array}{l}\text { 72.08078(100), 77.03858(42), } \\
\text { 79.05423(32), 105.06988(31), } \\
\text { 103.05423(29), 91.05423(15), } \\
135.08044(12)\end{array}$ \\
\hline \multirow[b]{2}{*}{$\begin{array}{l}\text { 4-Methylethcathinone } \\
\text { metabolite }(( \pm) \text {-Ephedrine } \\
\text { stereochemistry })\end{array}$} & 10 & $176.14338(100), 194.15394(16)$ \\
\hline & 20 & $\begin{array}{l}\text { 176.14338(100), 131.08553(43), } \\
91.05423(28), 147.10425(22), \\
161.1199(21), 146.09643(15), \\
105.06988(13), 148.11208(12)\end{array}$ \\
\hline
\end{tabular}




\begin{tabular}{|c|c|c|}
\hline & 40 & $\begin{array}{l}\text { 91.05423(100), 115.05423(45), } \\
116.06205(36), 131.07295(26), \\
\text { 146.09643(20), 105.06988(19), } \\
\text { 130.06513(19), 129.06988(18), } \\
\text { 144.08078(16), 43.01784(12), } \\
\text { 42.03383(10), 128.06205(10) }\end{array}$ \\
\hline \multirow{3}{*}{ N-ethyl-N-Methylcathinone } & 10 & $\begin{array}{l}\text { 192.13829(100), 133.06479(20), } \\
105.06988(19), 86.09643(11)\end{array}$ \\
\hline & 20 & $\begin{array}{l}\text { 105.06988(100), 86.09643(62), } \\
\text { 133.06479(29), 192.13829(26), } \\
\text { 58.06513(12) }\end{array}$ \\
\hline & 40 & $\begin{array}{l}77.03858(100), 105.06988(49), \\
58.06513(49), 86.09643(47), \\
79.05423(39), 103.05423(26), \\
105.03349(12), 44.04948(12), \\
130.06513(10)\end{array}$ \\
\hline \multirow{3}{*}{ Isopentedrone } & 10 & $\begin{array}{l}\text { 91.05423(100), 192.13829(70), } \\
161.09609(62), 174.12773(45), \\
132.08078(13), 119.04914(12)\end{array}$ \\
\hline & 20 & $91.05423(100)$ \\
\hline & 40 & $91.05423(100), 65.03858(17)$ \\
\hline \multirow{3}{*}{$\begin{array}{l}\text { Mephedrone metabolite }(( \pm)- \\
\text { Ephedrine stereochemistry) }\end{array}$} & 10 & $149.09609(100), 121.06479(38)$ \\
\hline & 20 & $\begin{array}{l}\text { 121.06479(100), 149.09609(15), } \\
91.05423(11)\end{array}$ \\
\hline & 40 & $\begin{array}{l}\text { 91.05423(100), 121.06479(72), } \\
77.03858(51), 78.0464(47), \\
65.03858(25)\end{array}$ \\
\hline \multirow{3}{*}{$\begin{array}{l}\text { Mephedrone metabolite }(( \pm)- \\
\text { Pseudoephedrine } \\
\text { stereochemistry) }\end{array}$} & 10 & $162.12773(100)$ \\
\hline & 20 & $\begin{array}{l}\text { 162.12773(100), 147.10425(72), } \\
91.05423(39), 131.08553(37), \\
\text { 105.06988(20), 116.06205(16), } \\
70.06513(13), 146.09643(12), \\
129.06988(12), 56.04948(11), \\
132.08078(11)\end{array}$ \\
\hline & 40 & $\begin{array}{l}\text { 91.05423(100), 115.05423(67), } \\
\text { 105.06988(63), 116.06205(34), } \\
77.03858(28), 146.09643(25), \\
56.04948(22), 131.07295(21), \\
132.08078(20), 79.05423(19), \\
103.05423(15), 42.03383(14), \\
65.03858(14), 128.06205(13), \\
129.06988(12), 130.06513(12)\end{array}$ \\
\hline
\end{tabular}




\begin{tabular}{|c|c|c|}
\hline \multirow{3}{*}{ NRG-3 } & 10 & $\begin{array}{l}224.14338(100), 242.15394(84), \\
211.11174(30), 182.09643(26), \\
141.06988(16), 181.0886(12)\end{array}$ \\
\hline & 20 & $\begin{array}{l}\text { 182.09643(100), 181.0886(84), } \\
141.06988(60), 224.14338(57), \\
167.07295(16), 211.1174(15), \\
180.08078(13), 194.09643(10)\end{array}$ \\
\hline & 40 & $\begin{array}{l}\text { 180.08078(100), 181.0886(80), } \\
\text { 141.06988(76), 127.05423(43), } \\
\text { 167.07295(42), 194.09643(40), } \\
115.05423(20), 166.06513(13)\end{array}$ \\
\hline \multirow[b]{3}{*}{$\begin{array}{l}\text { Pentedrone metabolite }(( \pm)- \\
\text { Ephedrine stereochemistry) }\end{array}$} & 10 & $176.14338(100), 194.15394(20)$ \\
\hline & 20 & $\begin{array}{l}176.14338(100), 133.0886(61), \\
91.05423(38), 117.06988(18), \\
134.09643(17), 145.10118(14), \\
132.08078(14), 120.08078(12)\end{array}$ \\
\hline & 40 & $\begin{array}{l}\text { 91.05423(100), 132.08078(33), } \\
77.03858(20), 56.04948(19), \\
\text { 115.05423(18), 79.05423(17), } \\
\text { 104.06205(16), 118.06513(16), } \\
\text { 43.05423(14), 133.0886(13), } \\
\text { 103.05423(12), 42.03383(12), } \\
\text { 65.03858(10) }\end{array}$ \\
\hline \multirow{3}{*}{$\begin{array}{l}\text { Pentedrone metabolite }(( \pm)- \\
\text { Pseudoephedrine } \\
\text { stereochemistry })\end{array}$} & 10 & $176.14338(100)$ \\
\hline & 20 & $\begin{array}{l}\text { 176.14338(100), 133.0886(68), } \\
91.05423(35), 134.09643(19), \\
\text { 117.06988(17), 132.08078(16), } \\
\text { 145.10118(14), 120.08078(12), } \\
\text { 119.07295(12) }\end{array}$ \\
\hline & 40 & $\begin{array}{l}\text { 91.05423(100), 132.08078(40), } \\
56.04948(21), 104.06205(20), \\
118.06513(20), 117.0573(16), \\
133.0886(16), 115.05423(16), \\
42.03383(13), 103.05423(12), \\
130.06513(12), 119.07295(11)\end{array}$ \\
\hline \multirow{3}{*}{ Benzedrone } & 10 & $\begin{array}{l}\text { 254.15394(100), 91.05423(69), } \\
236.14338(20)\end{array}$ \\
\hline & 20 & $91.05423(100)$ \\
\hline & 40 & $91.05423(100)$ \\
\hline \multirow[t]{2}{*}{ (-)-(S)-Cathinone } & 10 & $\begin{array}{l}\text { 132.08078(100), 133.06479(20), } \\
\text { 105.06988(20), 117.0573(18), } \\
150.09134(12)\end{array}$ \\
\hline & 20 & $\begin{array}{l}\text { 117.0573(100), 105.06988(46), } \\
132.08078(44)\end{array}$ \\
\hline
\end{tabular}




\begin{tabular}{|c|c|c|}
\hline & 40 & $\begin{array}{l}\text { 77.03858(100), 90.0464(81), } \\
117.0573(80), 89.03858(53), \\
51.02293(27), 79.05423(22), \\
103.05423(14), 130.06513(11)\end{array}$ \\
\hline \multirow{3}{*}{ 2,3-Dimethylethcathinone } & 10 & $\begin{array}{l}\text { 188.14338(100), 206.15394(46), } \\
159.10425(11), 173.1199(10), \\
160.11208(10)\end{array}$ \\
\hline & 20 & $\begin{array}{l}\text { 173.1199(100), 159.10425(99), } \\
\text { 188.14338(71), 158.09643(53), } \\
\text { 160.11208(46), 145.0886(23), } \\
133.10118(21), 144.08078(14)\end{array}$ \\
\hline & 40 & $\begin{array}{l}\text { 158.09643(100), 144.08078(51), } \\
\text { 143.07295(18), 105.06988(17), } \\
91.05423(15), 115.05423(12), \\
145.0886(10)\end{array}$ \\
\hline \multirow{3}{*}{ 2,4-Dimethylethcathinone } & 10 & $188.14338(100), 206.15394(37)$ \\
\hline & 20 & $\begin{array}{l}\text { 173.1199(100), 159.10425(99), } \\
\text { 188.14338(79), 158.09643(48), } \\
\text { 160.11208(45), 145.0886(22), } \\
133.10118(22), 72.08078(20), \\
144.08078(12)\end{array}$ \\
\hline & 40 & $\begin{array}{l}\text { 158.09643(100), 144.08078(45), } \\
\text { 105.06988(17), 143.07295(16), } \\
91.05423(16), 115.05423(13), \\
128.06205(10)\end{array}$ \\
\hline \multirow{3}{*}{ Diethylcathinone } & 10 & $\begin{array}{l}\text { 188.14338(100), 206.15394(59), } \\
\text { 159.10425(16), 173.1199(14), } \\
160.11208(12)\end{array}$ \\
\hline & 20 & $\begin{array}{l}\text { 188.14338(100), 159.10425(95), } \\
\text { 173.1199(90), 158.09643(62), } \\
\text { 160.11208(45), 133.10118(28), } \\
\text { 145.0886(25), 144.08078(17), } \\
105.06988(10)\end{array}$ \\
\hline & 40 & $\begin{array}{l}\text { 158.09643(100), 144.08078(47), } \\
\text { 105.06988(22), 91.05423(21), } \\
\text { 115.05423(18), 143.07295(18), } \\
145.0886(11), 117.06988(11)\end{array}$ \\
\hline \multirow[b]{3}{*}{ 2,3-Dimethylmethcathinone } & 10 & $161.09609(100), 133.06479(15)$ \\
\hline & 20 & $133.06479(100), 161.09609(50)$ \\
\hline & 40 & $\begin{array}{l}133.06479(100), 105.06988(69), \\
77.03858(67), 79.05423(62), \\
103.05423(47), 91.05423(40), \\
117.06988(23), 115.05423(20), \\
128.06205(17), 146.07262(14),\end{array}$ \\
\hline
\end{tabular}




\begin{tabular}{|c|c|c|}
\hline & & $\begin{array}{l}55.01784(11), 131.04914(11) \\
120.05697(10)\end{array}$ \\
\hline \multirow{3}{*}{ 2,4-Dimethylmethcathinone } & 10 & $\begin{array}{l}\text { 174.12773(100), 192.13829(26), } \\
\text { 161.09609(21), 145.0886(14), } \\
146.09643(13)\end{array}$ \\
\hline & 20 & $\begin{array}{l}\text { 145.0886(100), 105.06988(45), } \\
174.12773(35), 146.09643(35) \\
144.08078(35), 159.10425(21)\end{array}$ \\
\hline & 40 & $\begin{array}{l}\text { 144.08078(100), 105.06988(18), } \\
91.05423(18)\end{array}$ \\
\hline \multirow[b]{3}{*}{$\begin{array}{l}\text { 3,4-Dimethylmethcathinone } \\
\text { metabolite }(( \pm) \text {-Ephedrine } \\
\text { stereochemistry })\end{array}$} & 10 & $176.14338(100)$ \\
\hline & 20 & $\begin{array}{l}\text { 176.14338(100), 161.1199(49), } \\
\text { 145.10118(28), 130.0777(15), } \\
\text { 105.06988(14), 56.04948(13), } \\
70.06513(13)\end{array}$ \\
\hline & 40 & $\begin{array}{l}\text { 105.06988(100), 129.06988(84), } \\
115.05423(78), 56.04948(66), \\
91.05423(57), 119.08553(57), \\
146.09643(47), 131.07295(46), \\
130.0777(44), 128.06205(41), \\
160.11208(35), 117.06988(29), \\
77.03858(25), 79.05423(25), \\
145.0886(24), 42.03383(23), \\
103.05423(17), 161.1199(14), \\
43.01784(14), 144.08078(12), \\
127.05423(12)\end{array}$ \\
\hline \multirow{3}{*}{$\begin{array}{l}\text { 3,4-Dimethylmethcathinone } \\
\text { metabolite }(( \pm) \text {-Pseudoephedrine } \\
\text { stereochemistry })\end{array}$} & 10 & $176.14338(100)$ \\
\hline & 20 & $\begin{array}{l}\text { 176.14338(100), 161.1199(51), } \\
\text { 145.10118(25), 70.06513(15), } \\
\text { 130.0777(14), 56.04948(13), } \\
105.06988(12)\end{array}$ \\
\hline & 40 & \\
\hline \multirow{3}{*}{ 3-Bromomethcathinone } & 10 & $145.0886(100), 242.0175(74)$ \\
\hline & 20 & $145.0886(100), 144.08078(23)$ \\
\hline & 40 & $144.08078(100)$ \\
\hline \multirow{4}{*}{ 4-Bromomethcathinone } & 10 & $145.0886(100), 242.0175(74)$ \\
\hline & 20 & $145.0886(100), 144.08078(23)$ \\
\hline & 40 & $144.08078(100)$ \\
\hline & 10 & $166.10265(100)$ \\
\hline
\end{tabular}




\begin{tabular}{|c|c|c|}
\hline \multirow{2}{*}{$\begin{array}{l}\text { 4-Fluoromethcathinone } \\
\text { metabolite }(( \pm) \text {-Ephedrine } \\
\text { stereochemistry })\end{array}$} & 20 & $\begin{array}{l}\text { 166.10265(100), 151.07918(70), } \\
\text { 135.06045(55), 115.05423(29), } \\
\text { 109.0448(19), 133.0448(12), } \\
\text { 70.06513(11) }\end{array}$ \\
\hline & 40 & $\begin{array}{l}\text { 109.0448(100), 133.0448(52), } \\
\text { 115.05423(46), 150.07135(16), } \\
\text { 122.05263(15), 83.02915(14), } \\
\text { 43.01784(10) }\end{array}$ \\
\hline \multirow{3}{*}{$\begin{array}{l}\text { 4-Fluoromethcathinone } \\
\text { metabolite (( } \pm \text {-Pseudoephedrine } \\
\text { stereochemistry) }\end{array}$} & 10 & $166.10265(100)$ \\
\hline & 20 & $\begin{array}{l}\text { 166.10265(100), 151.07918(70), } \\
\text { 135.06045(55), 115.05423(29), } \\
\text { 109.0448(19), 133.0448(12), } \\
70.06513(11)\end{array}$ \\
\hline & 40 & $\begin{array}{l}\text { 109.0448(100), 133.0448(52), } \\
\text { 115.05423(46), 150.07135(16), } \\
\text { 122.05263(15), 83.02915(14), } \\
\text { 43.01784(10) }\end{array}$ \\
\hline \multirow{3}{*}{$\begin{array}{c}(-)-3,4-\text { Methylenedioxy } \\
\text { Pyrovalerone }\end{array}$} & 10 & $276.15942(100)$ \\
\hline & 20 & $\begin{array}{l}\text { 276.15942(100), 175.07536(63), } \\
\text { 205.08592(62), 126.12773(62), } \\
135.04406(49), 149.02332(26)\end{array}$ \\
\hline & 40 & $\begin{array}{l}126.12773(100), 135.04406(82), \\
\text { 149.02332(70), 84.08078(34), } \\
\text { 121.02841(31), 65.03858(15), } \\
175.07536(14), 133.02841(10)\end{array}$ \\
\hline \multirow{3}{*}{$\begin{array}{c}(+)-3,4-\text { Methylenedioxy } \\
\text { Pyrovalerone }\end{array}$} & 10 & $276.15942(100)$ \\
\hline & 20 & $\begin{array}{l}\text { 276.15942(100), 205.08592(61), } \\
\text { 126.12773(60), 175.07536(60), } \\
\text { 135.04406(49), 149.02332(25) }\end{array}$ \\
\hline & 40 & $\begin{array}{l}\text { 126.12773(100), 135.04406(81), } \\
\text { 149.02332(70), 84.08078(33), } \\
121.02841(32), 65.03858(15), \\
175.07536(15), 133.02841(11)\end{array}$ \\
\hline \multirow{3}{*}{ 2,3-MDA } & 10 & $\begin{array}{l}\text { 163.07536(100), 135.04406(71), } \\
\text { 133.06479(16), 105.06988(15), } \\
\text { 180.10191(11) }\end{array}$ \\
\hline & 20 & $\begin{array}{l}135.04406(100), 105.06988(69) \\
133.06479(20), 79.05423(12)\end{array}$ \\
\hline & 40 & $\begin{array}{l}\text { 77.03858(100), 79.05423(40), } \\
\text { 105.06988(21), 51.02293(19), } \\
103.05423(19)\end{array}$ \\
\hline 2,3-MDMA & 10 & $\begin{array}{l}163.07536(100), 194.11756(53), \\
135.04406(52), 133.06479(11)\end{array}$ \\
\hline
\end{tabular}




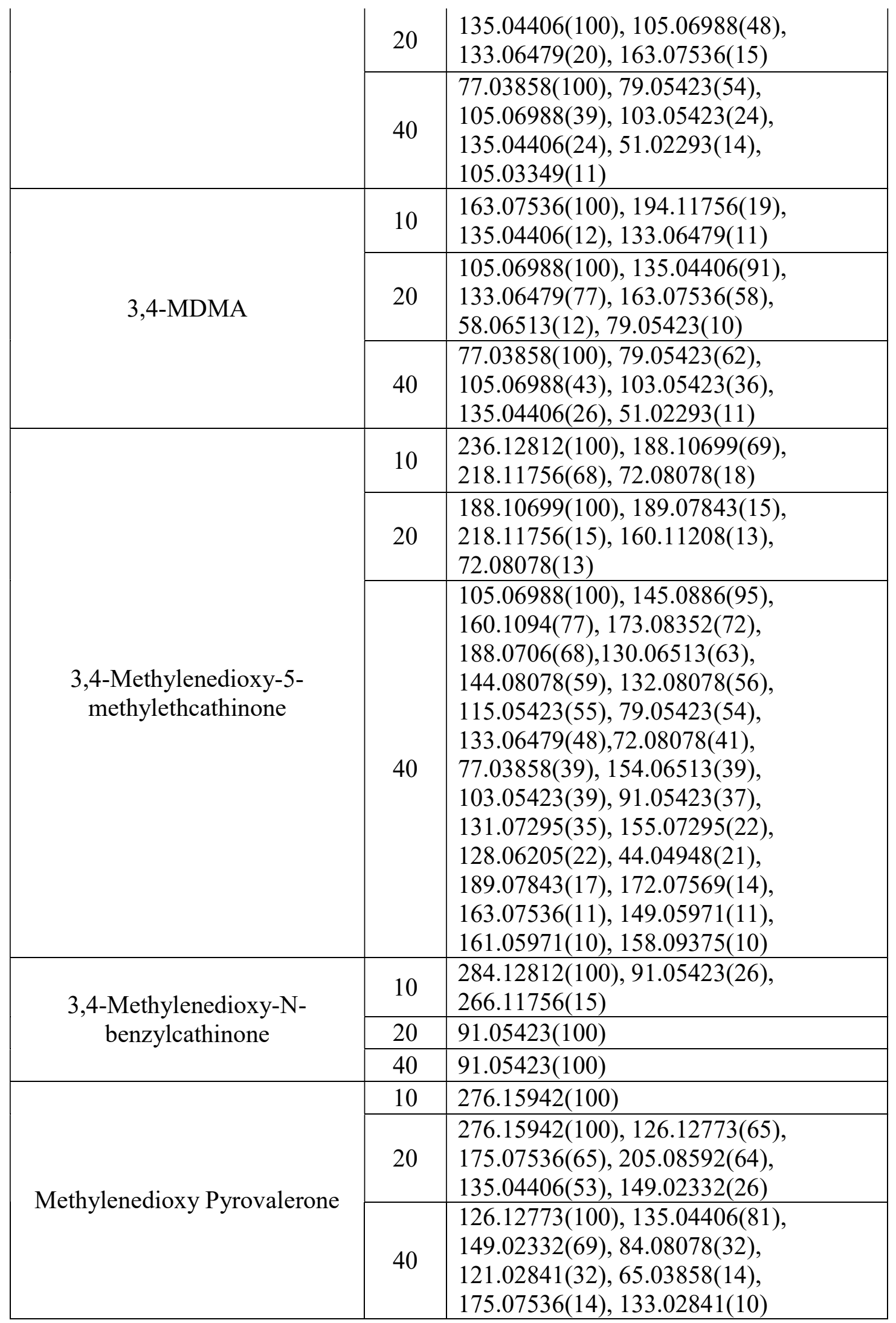




\begin{tabular}{|c|c|c|}
\hline \multirow{3}{*}{$\begin{array}{c}\text { Methylenedioxy Pyrovalerone } \\
\text { Metabolite } 1\end{array}$} & 10 & $278.17507(100)$ \\
\hline & 20 & $\begin{array}{l}\text { 175.07536(100), 278.17507(95), } \\
126.12773(83), 72.08078(38), \\
207.10157(30), 137.05971(30), \\
151.03897(30)\end{array}$ \\
\hline & 40 & \\
\hline \multirow{3}{*}{$\begin{array}{l}\text { Methylenedioxy Pyrovalerone } \\
\text { metabolite } 2\end{array}$} & 10 & $264.15942(100)$ \\
\hline & 20 & $\begin{array}{l}\text { 264.15942(100), 72.08078(97), } \\
\text { 126.12773(69), 123.04406(69), } \\
\text { 193.08592(42), 137.02332(30), } \\
175.07536(29)\end{array}$ \\
\hline & 40 & $\begin{array}{l}\text { 123.04406(100), 126.12773(93), } \\
\text { 137.02332(67), 72.08078(41), } \\
84.08078(39), 109.02841(36), \\
81.03349(17), 97.0886(10)\end{array}$ \\
\hline \multirow{3}{*}{$\begin{array}{c}\mathrm{N} \text {-acetyl-3,4- } \\
\text { Methylenedioxymethcathinone }\end{array}$} & 10 & $\begin{array}{l}\text { 208.09682(100), 250.10738(49), } \\
190.08626(13), 160.07569(12)\end{array}$ \\
\hline & 20 & $\begin{array}{l}160.07569(100), 190.08626(61) \\
208.09682(48), 58.06513(21)\end{array}$ \\
\hline & 40 & $\begin{array}{l}\text { 132.08078(100), 160.07569(82), } \\
58.06513(21), 117.0573(19), \\
91.05423(13)\end{array}$ \\
\hline \multirow{3}{*}{ N-hydroxy MDA } & 10 & $\begin{array}{l}\text { 163.07536(100), 135.04406(16), } \\
133.06479(14)\end{array}$ \\
\hline & 20 & $\begin{array}{l}\text { 105.06988(100), 135.04406(87), } \\
\text { 133.06479(78), 163.07536(43), } \\
79.05423(15)\end{array}$ \\
\hline & 40 & $\begin{array}{l}\text { 77.03858(100), 79.05423(72), } \\
\text { 103.05423(41), 105.06988(32), } \\
\text { 135.04406(22), 51.02293(17) }\end{array}$ \\
\hline \multirow{3}{*}{ 25C-NBOMe } & 10 & $336.1361(100), 121.06479(51)$ \\
\hline & 20 & $121.06479(100), 91.05423(11)$ \\
\hline & 40 & $\begin{array}{l}\text { 91.05423(100), 121.06479(23), } \\
\text { 93.06988(12) }\end{array}$ \\
\hline \multirow{3}{*}{ 25D-NBOMe } & 10 & $316.19072(100), 121.06479(26)$ \\
\hline & 20 & $121.06479(100), 91.05423(12)$ \\
\hline & 40 & $\begin{array}{l}\text { 91.05423(100), 121.06479(19), } \\
\text { 93.06988(11) }\end{array}$ \\
\hline \multirow{3}{*}{ 25E-NBOMe } & 10 & $330.20637(100), 121.06479(20)$ \\
\hline & 20 & $\begin{array}{l}\text { 121.06479(100), 193.12231(12), } \\
91.05423(10)\end{array}$ \\
\hline & 40 & $\begin{array}{l}\text { 91.05423(100), 121.06479(25), } \\
93.06988(13)\end{array}$ \\
\hline
\end{tabular}




\begin{tabular}{|c|c|c|}
\hline \multirow{3}{*}{ 25G-NBOMe } & 10 & $330.20637(100), 121.06479(20)$ \\
\hline & 20 & $\begin{array}{l}\text { 121.06479(100), 193.12231(12), } \\
91.05423(10)\end{array}$ \\
\hline & 40 & $\begin{array}{l}\text { 91.05423(100), 121.06479(25), } \\
\text { 93.06988(13) }\end{array}$ \\
\hline \multirow{3}{*}{ 25H-NBOMe } & 10 & $302.17507(100), 121.06479(45)$ \\
\hline & 20 & $121.06479(100), 91.05423(14)$ \\
\hline & 40 & $91.05423(100), 121.06479(15)$ \\
\hline \multirow[b]{3}{*}{ 25I-NBF } & 10 & $416.05173(100)$ \\
\hline & 20 & $\begin{array}{l}290.98765(100), 275.96417(26), \\
416.05173(23)\end{array}$ \\
\hline & 40 & $\begin{array}{l}\text { 275.96417(100), 260.9407(54), } \\
\text { 109.0448(53), 149.05971(30), } \\
\text { 134.07262(26), 164.08318(19), } \\
290.98765(19), 104.06205(15), \\
121.06479(13), 91.05423(10)\end{array}$ \\
\hline \multirow{3}{*}{ 25I-NBOMe 3-methoxy isomer } & 10 & $121.06479(100), 428.07171(32)$ \\
\hline & 20 & $121.06479(100)$ \\
\hline & 40 & $121.06479(100)$ \\
\hline \multirow{3}{*}{ 25I-NBOMe 4-methoxy isomer } & 10 & $428.07171(100), 290.98765(11)$ \\
\hline & 20 & $\begin{array}{l}121.06479(100), 290.98765(72), \\
272.1407(47), 428.07171(44), \\
275.96417(16)\end{array}$ \\
\hline & 40 & $\begin{array}{l}121.06479(100), 275.96417(23), \\
91.05423(22)\end{array}$ \\
\hline \multirow[b]{3}{*}{ 25I-NBOMe imine analog } & 10 & $426.05606(100)$ \\
\hline & 20 & $\begin{array}{l}\text { 426.05606(100), 290.98765(65), } \\
275.96417(21)\end{array}$ \\
\hline & 40 & $\begin{array}{l}275.96417(100), 260.9407(46), \\
149.05971(28), 290.98765(24), \\
134.07262(24), 164.08318(20), \\
121.06479(12)\end{array}$ \\
\hline \multirow{3}{*}{ 25T2-NBOMe } & 10 & $348.16279(100), 121.06479(21)$ \\
\hline & 20 & $121.06479(100), 211.07536(13)$ \\
\hline & 40 & $\begin{array}{l}\text { 91.05423(100), 121.06479(33), } \\
\text { 93.06988(15) }\end{array}$ \\
\hline \multirow{3}{*}{ 30C-NBOMe } & 10 & $181.08592(100)$ \\
\hline & 20 & $181.08592(100)$ \\
\hline & 40 & $181.08592(100), 148.05188(29)$ \\
\hline \multirow[b]{2}{*}{ 3-methoxy PCP } & 10 & $86.09643(100), 189.12739(60)$ \\
\hline & 20 & $\begin{array}{l}86.09643(100), 121.06479(80), \\
189.12739(36), 81.06988(19)\end{array}$ \\
\hline
\end{tabular}




\begin{tabular}{|c|c|c|}
\hline & 40 & $\begin{array}{l}121.06479(100), 86.09643(57), \\
91.05423(24), 81.06988(16)\end{array}$ \\
\hline \multirow{3}{*}{ 4-methoxy PCP } & 10 & $\begin{array}{l}\text { 189.12739(100), 86.09643(23), } \\
121.06479(12)\end{array}$ \\
\hline & 20 & $\begin{array}{l}121.06479(100), 189.12739(62), \\
86.09643(23)\end{array}$ \\
\hline & 40 & $121.06479(100)$ \\
\hline \multirow{3}{*}{ PCEEA } & 10 & $\begin{array}{l}\text { 159.11683(100), 90.09134(84), } \\
91.05423(20)\end{array}$ \\
\hline & 20 & $\begin{array}{l}\text { 91.05423(100), 159.11683(32), } \\
90.09134(23), 44.04948(19), \\
81.06988(19)\end{array}$ \\
\hline & 40 & $91.05423(100), 44.04948(10)$ \\
\hline \multirow{3}{*}{ PCMPA } & 10 & $\begin{array}{l}90.09134(100), 159.11683(99), \\
91.05423(17), 248.20089(11)\end{array}$ \\
\hline & 20 & $\begin{array}{l}91.05423(100), 159.11683(45), \\
90.09134(42), 81.06988(19), \\
58.06513(17)\end{array}$ \\
\hline & 40 & $91.05423(100)$ \\
\hline \multirow{3}{*}{$\mathrm{PCPr}$} & 10 & $\begin{array}{l}\text { 159.11683(100), 60.08078(44), } \\
91.05423(32), 81.06988(11)\end{array}$ \\
\hline & 20 & $\begin{array}{l}\text { 91.05423(100), 60.08078(18), } \\
159.11683(14), 81.06988(13)\end{array}$ \\
\hline & 40 & $91.05423(100)$ \\
\hline \multirow{3}{*}{ Benocyclidine } & 10 & $215.0889(100), 86.09643(39)$ \\
\hline & 20 & $\begin{array}{l}\text { 215.0889(100), 147.0263(86), } \\
86.09643(49), 81.06988(11)\end{array}$ \\
\hline & 40 & $147.0263(100), 86.09643(17)$ \\
\hline \multirow[b]{3}{*}{$\begin{array}{l}\text { 2-methyl- } \alpha- \\
\text { Pyrrolidinopropiophenone }\end{array}$} & 10 & $218.15394(100)$ \\
\hline & 20 & $\begin{array}{l}\text { 119.08553(100), 218.15394(83), } \\
\text { 147.08044(65), 98.09643(56), } \\
70.06513(19)\end{array}$ \\
\hline & 40 & $\begin{array}{l}\text { 98.09643(100), 91.05423(99), } \\
\text { 119.08553(55), 117.06988(36), } \\
56.04948(27), 70.06513(17), \\
\text { 84.08078(15), 103.05423(14), } \\
55.05423(13), 77.03858(13), \\
104.06205(10)\end{array}$ \\
\hline \multirow[b]{2}{*}{$\begin{array}{l}\text { 3,4-dimethoxy- } \alpha \text { - } \\
\text { Pyrrolidinopentiophenone }\end{array}$} & 10 & $292.19072(100)$ \\
\hline & 20 & $\begin{array}{l}221.11722(100), 151.07536(87), \\
292.19072(83), 126.12773(82), \\
165.05462(25), 193.12231(12)\end{array}$ \\
\hline
\end{tabular}




\begin{tabular}{|c|c|c|}
\hline & 40 & $\begin{array}{l}\text { 151.07536(100), 126.12773(80), } \\
\text { 165.05462(32), 84.08078(23), } \\
137.05971(12)\end{array}$ \\
\hline \multirow{3}{*}{$\begin{array}{l}\text { 3'-fluoro- } \alpha \text { - } \\
\text { Pyrrolidinopropiophenone }\end{array}$} & 10 & $222.12887(100)$ \\
\hline & 20 & $\begin{array}{l}\text { 222.12887(100), 98.09643(69), } \\
123.06045(57), 70.06513(27), \\
151.05537(26)\end{array}$ \\
\hline & 40 & $\begin{array}{l}98.09643(100), 103.05423(68), \\
77.03858(28), 70.06513(27), \\
95.02915(27), 56.04948(24), \\
123.06045(21), 84.08078(17), \\
123.02407(14), 55.05423(11)\end{array}$ \\
\hline \multirow[b]{3}{*}{$\begin{array}{l}\text { 3-methyl- } \alpha \text { - } \\
\text { Pyrrolidinopropiophenone }\end{array}$} & 10 & $218.15394(100)$ \\
\hline & 20 & $\begin{array}{l}\text { 119.08553(100), 218.15394(83), } \\
\text { 147.08044(65), 98.09643(56), } \\
70.06513(19)\end{array}$ \\
\hline & 40 & $\begin{array}{l}\text { 98.09643(100), 91.05423(99), } \\
\text { 119.08553(55), 117.06988(36), } \\
\text { 56.04948(27), 70.06513(17), } \\
\text { 84.08078(15), 103.05423(14), } \\
\text { 55.05423(13), 77.03858(13), } \\
\text { 104.06205(10) }\end{array}$ \\
\hline \multirow{3}{*}{$\begin{array}{l}\text { 4'-Methyl-N- } \\
\text { methylhexanophenone }\end{array}$} & 10 & $\begin{array}{l}\text { 202.15903(100), 220.16959(54), } \\
\text { 189.12739(29), 146.09643(18), } \\
\text { 105.06988(13) }\end{array}$ \\
\hline & 20 & $\begin{array}{l}\text { 146.09643(100), 105.06988(63), } \\
\text { 145.0886(53), 202.15903(49), } \\
\text { 131.07295(16), 144.08078(14), } \\
\text { 119.04914(13), 158.09643(13), } \\
159.10425(12)\end{array}$ \\
\hline & 40 & $\begin{array}{l}\text { 144.08078(100), 105.06988(50), } \\
\text { 158.09643(45), 91.05423(38), } \\
\text { 145.0886(31), 131.07295(30), } \\
\text { 130.06513(18), 77.03858(11), } \\
79.05423(11)\end{array}$ \\
\hline \multirow{4}{*}{$\begin{array}{l}\text { 4-fluoro- } \alpha- \\
\text { Pyrrolidinobutiophenone }\end{array}$} & 10 & $236.14452(100)$ \\
\hline & 20 & $\begin{array}{l}\text { 109.0448(100), 236.14452(85), } \\
\text { 165.07102(56), 112.11208(51), } \\
\text { 137.0761(40), 123.02407(26), } \\
70.06513(20)\end{array}$ \\
\hline & 40 & $\begin{array}{l}\text { 109.0448(100), 112.11208(60), } \\
95.02915(46), 123.02407(45), \\
84.08078(27), 70.06513(17)\end{array}$ \\
\hline & 10 & $250.16017(100)$ \\
\hline
\end{tabular}




\begin{tabular}{|c|c|c|}
\hline \multirow{2}{*}{$\begin{array}{l}\text { 4-fluoro- } \alpha \text { - } \\
\text { Pyrrolidinopentiophenone }\end{array}$} & 20 & $\begin{array}{l}109.0448(100), 250.16017(79), \\
126.12773(40), 179.08667(39), \\
123.02407(23), 70.06513(14)\end{array}$ \\
\hline & 40 & $\begin{array}{l}\text { 109.0448(100), 126.12773(57), } \\
\text { 123.02407(53), 95.02915(48), } \\
84.08078(33), 70.06513(11)\end{array}$ \\
\hline \multirow[b]{3}{*}{$\begin{array}{l}\text { 4'-fluoro- } \alpha \text { - } \\
\text { Pyrrolidinopropiophenone }\end{array}$} & 10 & $222.12887(100)$ \\
\hline & 20 & $\begin{array}{l}222.12887(100), 123.06045(85), \\
98.09643(82), 151.05537(64), \\
70.06513(19)\end{array}$ \\
\hline & 40 & $\begin{array}{l}\text { 98.09643(100), 103.05423(85), } \\
\text { 123.06045(34), 77.03858(30), } \\
\text { 56.04948(27), 70.06513(18), } \\
\text { 95.02915(17), 123.02293(14), } \\
\text { 84.08078(14), 55.05423(11) }\end{array}$ \\
\hline \multirow{3}{*}{$\begin{array}{l}\text { 4-Methyl- } \alpha \text { - } \\
\text { ethylaminobutiophenone }\end{array}$} & 10 & $\begin{array}{l}188.14338(100), 206.15394(72), \\
161.09609(20), 160.11208(16), \\
159.10425(13)\end{array}$ \\
\hline & 20 & $\begin{array}{l}159.10425(100), 105.06988(68), \\
144.08078(62), 188.14338(55), \\
160.11208(54), 132.08078(24), \\
131.07295(11), 158.09643(10)\end{array}$ \\
\hline & 40 & $\begin{array}{l}144.08078(100), 105.06988(40), \\
91.05423(33), 130.06513(18), \\
143.07295(12), 158.09643(11)\end{array}$ \\
\hline \multirow{3}{*}{$\begin{array}{l}\text { 4-Methyl- } \alpha \text { - } \\
\text { ethylaminopentiophenone }\end{array}$} & 10 & $\begin{array}{l}202.15903(100), 220.16959(79), \\
175.11174(27), 160.11208(12), \\
105.06988(12)\end{array}$ \\
\hline & 20 & $\begin{array}{l}105.06988(100), 202.15903(81), \\
160.11208(81), 159.10425(69), \\
144.08078(58), 132.08078(50), \\
173.1199(33), 174.12773(27), \\
119.04914(20), 131.07295(14), \\
175.11174(14), 158.09643(14), \\
145.0886(11)\end{array}$ \\
\hline & 40 & $\begin{array}{l}144.08078(100), 105.06988(39), \\
91.05423(31), 130.06513(14), \\
158.09643(12), 117.0573(10)\end{array}$ \\
\hline$\alpha$-Ethylaminopentiophenone & 10 & $\begin{array}{l}\text { 188.14338(100), 206.15394(99), } \\
146.09643(21), 161.09609(18), \\
91.05423(15)\end{array}$ \\
\hline
\end{tabular}




\begin{tabular}{|c|c|c|}
\hline & 20 & $\begin{array}{l}91.05423(100), 146.09643(83), \\
118.06513(67), 188.14338(49), \\
130.06513(48), 145.0886(38), \\
159.10425(24), 105.03349(23), \\
160.11208(21), 117.0573(12), \\
131.07295(11)\end{array}$ \\
\hline & 40 & $\begin{array}{l}\text { 130.06513(100), 91.05423(82), } \\
\text { 77.03858(53), 117.0573(21), } \\
118.06513(17), 105.03349(11)\end{array}$ \\
\hline \multirow{3}{*}{$\alpha$-Pyrrolidinobutiothiophenone } & 10 & $224.11036(100), 112.11208(20)$ \\
\hline & 20 & $\begin{array}{l}112.11208(100), 125.04195(33), \\
224.11036(19), 153.03686(16), \\
97.01065(10)\end{array}$ \\
\hline & 40 & $\begin{array}{l}\text { 112.11208(100), 97.01065(45), } \\
\text { 110.98991(42), 70.06513(22), } \\
84.08078(16), 55.05423(15)\end{array}$ \\
\hline \multirow[b]{3}{*}{$\begin{array}{l}\alpha \text {-Pyrrolidinopentiophenone } \\
\text { metabolite } 1\end{array}$} & 10 & $\begin{array}{l}\text { 234.18524(100), 216.17468(23), } \\
72.08078(14)\end{array}$ \\
\hline & 20 & $\begin{array}{l}72.08078(100), 216.17468(76) \\
173.1199(26), 234.18524(19), \\
91.05423(16), 145.10118(15) \\
\end{array}$ \\
\hline & 40 & $\begin{array}{l}\text { 72.08078(100), 91.05423(69), } \\
\text { 79.05423(43), 43.05423(38), } \\
\text { 172.11208(20), 103.05423(17), } \\
\text { 104.06205(14), 117.06988(13), } \\
\text { 77.03858(13), 41.03858(12), } \\
\text { 105.06988(11) }\end{array}$ \\
\hline \multirow{3}{*}{$\alpha$-Pyrrolidinopentiothiophenone } & 10 & $238.12601(100), 126.12773(17)$ \\
\hline & 20 & $\begin{array}{l}\text { 126.12773(100), 97.01065(43), } \\
238.12601(22), 167.05251(12)\end{array}$ \\
\hline & 40 & $\begin{array}{l}\text { 126.12773(100), 97.01065(60), } \\
\text { 110.98991(41), 84.08078(33), } \\
97.0886(20), 55.05423(10)\end{array}$ \\
\hline \multirow{3}{*}{ 4-fluoro PV8 } & 10 & $278.19147(100)$ \\
\hline & 20 & $\begin{array}{l}\text { 278.19147(100), 109.0448(69), } \\
\text { 154.15903(24), 207.11683(16), } \\
\text { 123.02407(12), 70.06513(11) }\end{array}$ \\
\hline & 40 & $\begin{array}{l}\text { 109.0448(100), 154.15903(48), } \\
\text { 123.02407(47), 84.08078(29), } \\
95.02915(25)\end{array}$ \\
\hline \multirow[b]{2}{*}{ 4-fluoro PV9 } & 10 & $292.20712(100)$ \\
\hline & 20 & $\begin{array}{l}\text { 292.20712(100), 109.0448(49), } \\
\text { 168.17468(16) }\end{array}$ \\
\hline
\end{tabular}




\begin{tabular}{|c|c|c|}
\hline & 40 & $\begin{array}{l}\text { 109.0448(100), 168.17468(49), } \\
\text { 123.02407(43), 84.08078(26), } \\
95.02915(17)\end{array}$ \\
\hline \multirow{3}{*}{ 4-methoxy PV8 } & 10 & $290.21146(100)$ \\
\hline & 20 & $\begin{array}{l}121.06479(100), 219.13796(98), \\
290.21146(96), 154.15903(76), \\
135.04406(25)\end{array}$ \\
\hline & 40 & $\begin{array}{l}121.06479(100), 154.15903(56), \\
135.04406(40), 84.08078(24), \\
77.03858(15)\end{array}$ \\
\hline \multirow{3}{*}{ 4-methoxy PV9 } & 10 & $304.22711(100)$ \\
\hline & 20 & $\begin{array}{l}304.22711(100), 233.15361(73), \\
121.06479(71), 168.17468(54), \\
135.04406(17)\end{array}$ \\
\hline & 40 & $\begin{array}{l}\text { 121.06479(100), 168.17468(59), } \\
135.04406(41), 84.08078(22), \\
77.03858(11)\end{array}$ \\
\hline \multirow{3}{*}{ PV8 } & 10 & $260.20089(100)$ \\
\hline & 20 & $\begin{array}{l}\text { 260.20089(100), 91.05423(75), } \\
\text { 154.15903(21), 70.06513(17), } \\
\text { 189.12739(15), 119.04914(14), } \\
\text { 105.03349(14) }\end{array}$ \\
\hline & 40 & $\begin{array}{l}\text { 91.05423(100), 77.03858(37), } \\
\text { 105.03349(35), 154.15903(35), } \\
\text { 84.08078(28) }\end{array}$ \\
\hline \multirow{3}{*}{ PV9 } & 10 & $274.21654(100)$ \\
\hline & 20 & $\begin{array}{l}\text { 274.21654(100), 91.05423(50), } \\
168.17468(14), 70.06513(12)\end{array}$ \\
\hline & 40 & $\begin{array}{l}\text { 91.05423(100), 105.03349(37), } \\
\text { 168.17468(35), 77.03858(28), } \\
84.08078(25), 70.06513(10)\end{array}$ \\
\hline \multirow[b]{3}{*}{ 4-APB } & 10 & $159.08044(100), 131.04914(73)$ \\
\hline & 20 & 131.04914(100), 91.05423(20) \\
\hline & 40 & $\begin{array}{l}\text { 91.05423(100), 77.03858(99), } \\
\text { 131.04914(80), 115.05423(42), } \\
\text { 103.05423(23), 116.06205(20), } \\
65.03858(19), 128.06205(11)\end{array}$ \\
\hline \multirow{3}{*}{ 4-APDB } & 10 & $161.09609(100), 133.06479(61)$ \\
\hline & 20 & $\begin{array}{l}133.06479(100), 161.09609(14), \\
120.05697(11)\end{array}$ \\
\hline & 40 & $\begin{array}{l}\text { 77.03858(100), 91.05423(64), } \\
79.05423(56), 103.05423(44), \\
105.06988(38), 133.06479(33)\end{array}$ \\
\hline
\end{tabular}




\begin{tabular}{|c|c|c|}
\hline & & $\begin{array}{l}\text { 115.05423(20), 117.06988(14), } \\
128.06205(11)\end{array}$ \\
\hline \multirow{3}{*}{ 5-APDB } & 10 & $161.09616(100), 178.12288(10)$ \\
\hline & 20 & $\begin{array}{l}\text { 161.09609(100), 105.06997(55), } \\
\text { 146.07258(42), 131.07957(18), } \\
91.05413(14)\end{array}$ \\
\hline & 40 & $\begin{array}{l}\text { 103.05423(100), 131.04918(51), } \\
91.05425(41), 77.03853(40), \\
115.05446(36)\end{array}$ \\
\hline \multirow{3}{*}{ 5-EAPB } & 10 & $\begin{array}{l}\text { 159.08044(100), 131.04914(35), } \\
204.13829(29)\end{array}$ \\
\hline & 20 & $131.04914(100), 159.08044(19)$ \\
\hline & 40 & $\begin{array}{l}\text { 131.04914(100), 91.05423(60), } \\
77.03858(29), 116.06205(17), \\
115.05423(15), 103.05423(15)\end{array}$ \\
\hline \multirow{3}{*}{ 5-MAPB } & 10 & $\begin{array}{l}\text { 159.08044(100), 131.04914(45), } \\
\text { 190.12264(19) }\end{array}$ \\
\hline & 20 & $\begin{array}{l}\text { 131.04914(100), 159.08044(13), } \\
91.05423(11)\end{array}$ \\
\hline & 40 & $\begin{array}{l}\text { 131.04914(100), 91.05423(77), } \\
77.03858(51), 115.05423(25), \\
116.06205(20), 103.05423(20)\end{array}$ \\
\hline \multirow[b]{3}{*}{ 5-MAPDB } & 10 & $161.09609(100), 133.06479(15)$ \\
\hline & 20 & $133.06479(100), 161.09609(50)$ \\
\hline & 40 & $\begin{array}{l}\text { 133.06479(100), 105.06988(69), } \\
77.03858(67), 79.05423(62), \\
103.05423(47), 91.05423(40), \\
117.06988(23), 115.05423(20), \\
128.06205(17), 146.07262(14), \\
55.01784(11), 131.04914(11), \\
120.05697(10)\end{array}$ \\
\hline \multirow[b]{3}{*}{ 6-APB } & 10 & $159.08044(100), 131.04914(68)$ \\
\hline & 20 & $131.04914(100), 91.05423(19)$ \\
\hline & 40 & $\begin{array}{l}\text { 91.05423(100), 77.03858(87), } \\
131.04914(78), 115.05423(48), \\
103.05423(23), 116.06205(23), \\
65.03858(16)\end{array}$ \\
\hline \multirow{2}{*}{ 6-APDB } & 10 & $161.09609(100), 133.06479(56)$ \\
\hline & 20 & $133.06479(100), 161.09609(16)$ \\
\hline
\end{tabular}




\begin{tabular}{|c|c|c|}
\hline & 40 & $\begin{array}{l}77.03858(100), 79.05423(64), \\
91.05423(52), 103.05423(52), \\
105.06988(50), 133.06479(48), \\
115.05423(21), 117.06988(18), \\
128.06205(15), 55.01784(10)\end{array}$ \\
\hline \multirow[b]{3}{*}{ 7-APB } & 10 & $131.04914(100), 159.08044(75)$ \\
\hline & 20 & $131.04914(100)$ \\
\hline & 40 & $\begin{array}{l}\text { 77.03858(100), 131.04914(70), } \\
91.05423(53), 115.05423(27), \\
103.05423(25), 116.06205(12)\end{array}$ \\
\hline \multirow{3}{*}{ 3-Methylbuphedrone } & 10 & $\begin{array}{l}\text { 174.12773(100), 192.13829(42), } \\
161.09609(20), 145.0886(15), \\
146.09643(14), 105.06988(10)\end{array}$ \\
\hline & 20 & $\begin{array}{l}145.0886(100), 105.06988(46), \\
146.09643(36), 144.08078(35), \\
174.12773(33), 159.10425(22), \\
131.07295(10)\end{array}$ \\
\hline & 40 & $\begin{array}{l}\text { 144.08078(100), 91.05423(20), } \\
105.06988(20)\end{array}$ \\
\hline \multirow{3}{*}{ 4-Fluorobuphedrone } & 10 & $\begin{array}{l}\text { 178.10265(100), 196.11322(37), } \\
165.07102(19), 150.07135(18), \\
149.06353(13)\end{array}$ \\
\hline & 20 & $\begin{array}{l}149.06353(100), 150.07135(51), \\
109.0448(49), 178.10265(37), \\
148.0557(15), 163.07918(12)\end{array}$ \\
\hline & 40 & $\begin{array}{l}\text { 148.0557(100), 109.0448(65), } \\
\text { 149.06353(37), 95.02915(33), } \\
\text { 108.03698(16), 135.04788(15), } \\
162.07135(12), 83.02915(11)\end{array}$ \\
\hline \multirow{3}{*}{ 4-Methylbuphedrone } & 10 & $\begin{array}{l}\text { 174.12773(100), 192.13829(26), } \\
161.09609(21), 145.0886(14), \\
146.09643(13)\end{array}$ \\
\hline & 20 & $\begin{array}{l}145.0886(100), 105.06988(45), \\
174.12773(35), 146.09643(35), \\
144.08078(35), 159.10425(21)\end{array}$ \\
\hline & 40 & $\begin{array}{l}144.08078(100), 105.06988(18), \\
91.05423(18)\end{array}$ \\
\hline \multirow[b]{2}{*}{ 4-methyl-N-Methylbuphedrone } & 10 & $\begin{array}{l}\text { 206.15394(100), 161.09609(56), } \\
105.06988(17), 133.10118(11)\end{array}$ \\
\hline & 20 & $\begin{array}{l}\text { 105.06988(100), 86.09643(37), } \\
119.04914(35), 161.09609(27), \\
133.10118(17)\end{array}$ \\
\hline
\end{tabular}




\begin{tabular}{|c|c|c|}
\hline & 40 & $\begin{array}{l}\text { 91.05423(100), 105.06988(78), } \\
\text { 86.09643(53), 71.07295(26), } \\
\text { 119.04914(21), 65.03858(16), } \\
79.05423(14), 77.03858(12)\end{array}$ \\
\hline \multirow{3}{*}{ N-Ethylbuphedrone } & 10 & $\begin{array}{l}\text { 174.12773(100), 192.13829(60), } \\
\text { 146.09643(25), 147.08044(16), } \\
91.05423(14), 145.0886(13)\end{array}$ \\
\hline & 20 & $\begin{array}{l}145.0886(100), 91.05423(99), \\
\text { 130.06513(82), 146.09643(76), } \\
\text { 174.12773(53), 118.06513(50), } \\
\text { 105.03349(17), 117.0573(15) }\end{array}$ \\
\hline & 40 & $\begin{array}{l}\text { 130.06513(100), 91.05423(72), } \\
77.03858(45), 117.0573(19)\end{array}$ \\
\hline \multirow{3}{*}{ Dimethocaine } & 10 & $\begin{array}{l}\text { 279.2067(100), 120.04439(12), } \\
142.15903(11)\end{array}$ \\
\hline & 20 & $\begin{array}{l}120.04439(100), 142.15903(47), \\
86.09643(31), 279.2067(14)\end{array}$ \\
\hline & 40 & $\begin{array}{l}\text { 120.04439(100), 86.09643(53), } \\
92.04948(36)\end{array}$ \\
\hline \multirow[t]{3}{*}{$( \pm)$-Cannabichromene } & 10 & $\begin{array}{l}\text { 315.23186(100), 193.12231(55), } \\
259.16926(44), 81.06988(32), \\
233.15361(30), 135.11683(14), \\
231.13796(10), 219.13796(10)\end{array}$ \\
\hline & 20 & \\
\hline & 40 & \\
\hline \multirow{3}{*}{$( \pm)-$ ORG 28611} & 10 & $270.14886(100), 384.26455(37)$ \\
\hline & 20 & $270.14886(100)$ \\
\hline & 40 & $\begin{array}{l}\text { 174.05495(100), 270.14886(33), } \\
55.05423(19)\end{array}$ \\
\hline \multirow{3}{*}{ 5-fluoro NNEI } & 10 & $375.18672(100), 232.11322(23)$ \\
\hline & 20 & $232.11322(100)$ \\
\hline & 40 & $232.11208(100), 144.04439(86)$ \\
\hline \multirow{3}{*}{$\begin{array}{l}\text { 5-fluoro NNEI 2'-naphthyl } \\
\text { isomer }\end{array}$} & 10 & $375.18672(100), 232.11322(31)$ \\
\hline & 20 & $232.11322(100)$ \\
\hline & 40 & $232.11322(100), 144.04439(86)$ \\
\hline \multirow{3}{*}{ 5-fluoro SDB-005 } & 10 & $233.10847(100)$ \\
\hline & 20 & $233.10847(100), 213.10224(26)$ \\
\hline & 40 & $\begin{array}{l}\text { 145.03964(100), 213.10224(24), } \\
69.06988(18), 177.04587(16)\end{array}$ \\
\hline \multirow[b]{2}{*}{ 5-fluoro SDB-006 } & 10 & $339.18672(100)$ \\
\hline & 20 & $\begin{array}{l}232.11322(100), 339.18672(89), \\
206.13395(71), 91.05423(40)\end{array}$ \\
\hline
\end{tabular}




\begin{tabular}{|c|c|c|}
\hline & 40 & $\begin{array}{l}91.05423(100), 144.04439(25), \\
232.11322(16), 118.06513(15)\end{array}$ \\
\hline \multirow[b]{3}{*}{ A-796260 } & 10 & $355.238(100), 125.09609(14)$ \\
\hline & 20 & $\begin{array}{l}\text { 125.09609(100), 114.09134(32), } \\
355.238(32)\end{array}$ \\
\hline & 40 & $\begin{array}{l}\text { 114.09134(100), 125.09609(51), } \\
55.05423(39), 97.10118(26), \\
70.06513(21), 57.06988(19), \\
69.06988(12)\end{array}$ \\
\hline \multirow[b]{3}{*}{ A-836339 } & 10 & $311.17878(100), 187.08996(63)$ \\
\hline & 20 & $187.08996(100), 125.09609(18)$ \\
\hline & 40 & $\begin{array}{l}\text { 187.08996(100), 59.04914(71), } \\
\text { 55.05423(56), 129.0481(42), } \\
\text { 125.09609(37), 57.06988(32), } \\
\text { 155.06375(32), 97.10118(23), } \\
69.06988(14)\end{array}$ \\
\hline \multirow{3}{*}{ JW 618} & 10 & $393.10322(100)$ \\
\hline & 20 & $393.10322(100)$ \\
\hline & 40 & $\begin{array}{l}\text { 169.0886(100), 393.10322(79), } \\
197.10732(26)\end{array}$ \\
\hline \multirow{3}{*}{ JW 642} & 10 & $463.14509(100), 183.08044(24)$ \\
\hline & 20 & $183.08044(100), 463.14509(30)$ \\
\hline & 40 & $\begin{array}{l}\text { 183.08044(100), 155.08553(15), } \\
165.06988(12), 168.05697(11)\end{array}$ \\
\hline \multirow{3}{*}{$\mathrm{MN}-25$} & 10 & $440.29077(100)$ \\
\hline & 20 & $\begin{array}{l}\text { 440.29077(100), 261.15975(34), } \\
114.09134(14)\end{array}$ \\
\hline & 40 & $\begin{array}{l}\text { 114.09134(100), 81.06988(26), } \\
\text { 176.10699(12), 261.15975(10) }\end{array}$ \\
\hline \multirow{3}{*}{ MN-25-2-methyl derivative } & 10 & $454.30642(100), 275.1754(12)$ \\
\hline & 20 & $\begin{array}{l}\text { 275.1754(100), 454.30642(96), } \\
114.09134(47)\end{array}$ \\
\hline & 40 & $114.09134(100)$ \\
\hline \multirow{3}{*}{ NNEI } & 10 & $357.19614(100), 214.12264(29)$ \\
\hline & 20 & $214.12264(100)$ \\
\hline & 40 & $\begin{array}{l}144.04439(100), 214.12264(86), \\
43.05423(14)\end{array}$ \\
\hline \multirow{3}{*}{ NNEI 2'-naphthyl isomer } & 10 & $357.19614(100), 214.12264(38)$ \\
\hline & 20 & $214.12264(100)$ \\
\hline & 40 & $\begin{array}{l}144.04439(100), 214.12264(82), \\
43.05423(13)\end{array}$ \\
\hline SDB-005 & 10 & $215.11789(100)$ \\
\hline
\end{tabular}




\begin{tabular}{|c|c|c|}
\hline & 20 & $215.11789(100)$ \\
\hline & 40 & $145.03964(100), 215.11789(17)$ \\
\hline \multirow{3}{*}{ SDB-006 } & 10 & $321.19614(100)$ \\
\hline & 20 & $\begin{array}{l}\text { 214.12264(100), 188.14338(63), } \\
321.19614(51), 91.05423(43), \\
132.08078(16)\end{array}$ \\
\hline & 40 & $\begin{array}{l}\text { 91.05423(100), 144.04439(24), } \\
118.06513(13), 132.08078(10)\end{array}$ \\
\hline \multirow{3}{*}{$\Delta 8-\mathrm{THC}$} & 10 & $315.23186(100)$ \\
\hline & 20 & $\begin{array}{l}\text { 315.23186(100), 193.12231(72), } \\
\text { 259.16926(44), 135.11683(40), } \\
93.06988(31), 233.15361(18), \\
\text { 181.12231(15), 235.16926(13), } \\
\text { 231.13796(13), 247.16926(13), } \\
\text { 207.13796(11), 107.08553(10), } \\
109.10118(10)\end{array}$ \\
\hline & 40 & \\
\hline \multirow{3}{*}{$\triangle 9-\mathrm{THC}$} & 10 & $315.23186(100)$ \\
\hline & 20 & $\begin{array}{l}\text { 315.23186(100), 193.12231(90), } \\
\text { 259.16926(47), 135.11683(42), } \\
93.06988(30), 221.15361(21), \\
\text { 235.16926(21), 233.15361(18), } \\
\text { 81.06988(17), 181.12231(16), } \\
\text { 109.10118(12), 123.04406(11), } \\
\text { 107.08553(11), 207.13796(11) }\end{array}$ \\
\hline & 40 & \\
\hline \multirow{3}{*}{ AM1248 azepane isomer } & 10 & $391.27439(100)$ \\
\hline & 20 & $\begin{array}{l}391.27439(100), 112.11208(58), \\
135.11683(23)\end{array}$ \\
\hline & 40 & $\begin{array}{l}112.11208(100), 135.11683(51), \\
58.06513(13)\end{array}$ \\
\hline \multirow{3}{*}{ AM2201 benzimidazole analog } & 10 & $361.17107(100)$ \\
\hline & 20 & $\begin{array}{l}\text { 361.17107(100), 233.10847(24), } \\
\text { 177.04587(17), 155.04914(15), } \\
273.10224(13)\end{array}$ \\
\hline & 40 & $\begin{array}{l}\text { 155.04914(100), 127.05423(65), } \\
177.04587(27), 145.03964(25), \\
129.04472(14)\end{array}$ \\
\hline \multirow{3}{*}{ KM 233} & 10 & $363.23186(100), 119.08553(37)$ \\
\hline & 20 & $119.08553(100), 363.23186(10)$ \\
\hline & 40 & $91.05423(100), 119.08553(84)$ \\
\hline LY2183240 & 10 & $\begin{array}{l}\text { 280.14444(100), 72.04439(48), } \\
167.08553(29), 87.05529(20)\end{array}$ \\
\hline
\end{tabular}




\begin{tabular}{|c|c|c|}
\hline & 20 & $\begin{array}{l}72.04439(100), 167.08553(84), \\
87.05529(13)\end{array}$ \\
\hline & 40 & $72.04439(100), 167.08553(37)$ \\
\hline \multirow{3}{*}{ LY2183240 2'-isomer } & 10 & $\begin{array}{l}\text { 280.14444(100), 72.04439(54), } \\
167.08553(32), 87.05529(19)\end{array}$ \\
\hline & 20 & $\begin{array}{l}\text { 72.04439(100), 167.08553(84), } \\
87.05529(16)\end{array}$ \\
\hline & 40 & $72.04439(100), 167.08553(39)$ \\
\hline \multirow{3}{*}{ SER-601 } & 10 & $435.3006(100)$ \\
\hline & 20 & $\begin{array}{l}\text { 435.3006(100), 135.11683(70), } \\
284.16451(25)\end{array}$ \\
\hline & 40 & $135.11683(100), 284.16451(20)$ \\
\hline \multirow{3}{*}{ Tetrahydrocannabivarin } & 10 & $287.20056(100)$ \\
\hline & 20 & \\
\hline & 40 & \\
\hline \multirow[b]{3}{*}{ Yangonin } & 10 & $259.09649(100), 231.10157(14)$ \\
\hline & 20 & $\begin{array}{l}\text { 161.05971(100), 231.10157(53), } \\
259.09649(40), \\
\text { 216.0781(31),199.07536(31), } \\
\text { 171.08044(28), 209.05971(24), } \\
\text { 198.06753(15), 203.10666(13), } \\
\text { 185.05971(13), 133.06479(10), } \\
213.09101(10)\end{array}$ \\
\hline & 40 & $\begin{array}{l}\text { 133.06479(100), 128.06205(67), } \\
\text { 68.99711(48), 118.04132(33), } \\
\text { 151.05423(31), 161.05971(29), } \\
\text { 139.05423(28), 115.05423(24), } \\
\text { 140.06205(23), 103.05423(22), } \\
\text { 171.08044(22), 79.05423(22), } \\
\text { 127.05423(21), 77.03858(20), } \\
\text { 141.06988(20), 155.04914(18), } \\
\text { 152.06205(18), 90.0464(18), } \\
\text { 156.05697(17), 157.06479(16), } \\
\text { 145.06479(15), 184.05188(13), } \\
129.06988(13), 144.05697(12), \\
173.05971(12), 142.04132(12), \\
183.04406(12), 168.05697(11)\end{array}$ \\
\hline \multirow{3}{*}{ Cannabidiolic Acid } & 10 & $341.21112(100)$ \\
\hline & 20 & $341.21112(100)$ \\
\hline & 40 & \\
\hline \multirow{3}{*}{ Cannabigerol } & 10 & $193.12231(100), 317.24751(15)$ \\
\hline & 20 & 193.12231(100) \\
\hline & 40 & \\
\hline
\end{tabular}




\begin{tabular}{|c|c|c|}
\hline \multirow{3}{*}{ EG-018 } & 10 & $392.20089(100), 155.04914(11)$ \\
\hline & 20 & $\begin{array}{l}155.04914(100), 392.20089(55), \\
264.13829(12)\end{array}$ \\
\hline & 40 & $155.04914(100), 127.05423(85)$ \\
\hline \multirow{3}{*}{$\begin{array}{l}( \pm)-J W H 018 \mathrm{~N}-(2- \\
\text { hydroxypentyl) metabolite }\end{array}$} & 10 & $358.18041(100)$ \\
\hline & 20 & $\begin{array}{l}358.17982(100), 155.04864(92), \\
230.11689(20)\end{array}$ \\
\hline & 40 & $\begin{array}{l}127.05377(100), 155.04848(86), \\
160.03941(16)\end{array}$ \\
\hline \multirow{3}{*}{$\begin{array}{l}( \pm)-J W H 018 \mathrm{~N}-(3- \\
\text { hydroxypentyl) metabolite }\end{array}$} & 10 & $358.18016(100), 155.04914(20)$ \\
\hline & 20 & $155.04914(100), 358.18016(18)$ \\
\hline & 40 & $127.05423(100), 155.04914(89)$ \\
\hline \multirow{3}{*}{$\begin{array}{l}( \pm)-J W H 018 \mathrm{~N}-(4- \\
\text { hydroxypentyl) metabolite }\end{array}$} & 10 & $358.1799(100), 155.04864(10)$ \\
\hline & 20 & $155.0487(100), 358.18016(46)$ \\
\hline & 40 & $127.05391(100), 155.04858(83)$ \\
\hline \multirow{3}{*}{$\begin{array}{l}\text { (R)-(-)-JWH } 018 \mathrm{~N}-(4- \\
\text { hydroxypentyl) metabolite }\end{array}$} & 10 & $358.18016(100), 155.04914(20)$ \\
\hline & 20 & $155.04914(100), 358.18016(18)$ \\
\hline & 40 & $127.05423(100), 155.04914(89)$ \\
\hline \multirow{3}{*}{$\begin{array}{l}(\mathrm{S})-(+)-J W H \text { 018 N-(4- } \\
\text { hydroxypentyl) metabolite }\end{array}$} & 10 & $358.18016(100), 155.04914(20)$ \\
\hline & 20 & $155.04914(100), 358.18016(18)$ \\
\hline & 40 & $127.05423(100), 155.04914(89)$ \\
\hline \multirow{3}{*}{$\begin{array}{l}\text { 5-fluoro JWH } 018 \text { adamantyl } \\
\text { analog }\end{array}$} & 10 & $368.23842(100)$ \\
\hline & 20 & $368.23842(100), 135.11683(22)$ \\
\hline & 40 & $\begin{array}{l}\text { 135.11683(100), 93.06988(13), } \\
\text { 107.08553(11) }\end{array}$ \\
\hline \multirow{3}{*}{$\begin{array}{l}\text { JWH } 018 \text { 2-hydroxyindole } \\
\text { metabolite }\end{array}$} & 10 & $358.18041(100)$ \\
\hline & 20 & $\begin{array}{l}358.17982(100), 155.04864(92), \\
230.11689(20)\end{array}$ \\
\hline & 40 & \\
\hline \multirow{3}{*}{$\begin{array}{c}\text { JWH } 018 \text { 4-hydroxyindole } \\
\text { metabolite }\end{array}$} & 10 & $358.18016(100)$ \\
\hline & 20 & $\begin{array}{l}358.18016(100), 155.04914(43), \\
230.11756(26)\end{array}$ \\
\hline & 40 & $\begin{array}{l}127.05423(100), 155.04914(86), \\
160.0393(42), 230.11756(24)\end{array}$ \\
\hline \multirow{3}{*}{$\begin{array}{c}\text { JWH } 018 \text { 5-hydroxyindole } \\
\text { metabolite }\end{array}$} & 10 & $358.18041(100)$ \\
\hline & 20 & $\begin{array}{l}358.17982(100), 155.04864(92), \\
230.11689(20)\end{array}$ \\
\hline & 40 & $\begin{array}{l}127.05377(100), 155.04848(86), \\
160.03941(16)\end{array}$ \\
\hline \multirow{3}{*}{$\begin{array}{c}\text { JWH } 018 \text { 6-hydroxyindole } \\
\text { metabolite }\end{array}$} & 10 & $358.17987(100)$ \\
\hline & 20 & $155.04841(100), 358.17954(66)$ \\
\hline & 40 & $127.05384(100), 155.04844(85)$ \\
\hline
\end{tabular}




\begin{tabular}{|c|c|c|}
\hline \multirow{3}{*}{$\begin{array}{l}\text { JWH } 018 \text { 7-hydroxyindole } \\
\text { metabolite }\end{array}$} & 10 & $358.18031(100), 155.04876(31)$ \\
\hline & 20 & $155.04881(100), 358.18008(14)$ \\
\hline & 40 & $\begin{array}{l}\text { 127.05397(100), 155.0488(91), } \\
\text { 144.04422(12) }\end{array}$ \\
\hline \multirow{3}{*}{$\begin{array}{l}\text { JWH } 018 \text { 8-quinolinyl } \\
\text { carboxamide }\end{array}$} & 10 & $214.1224(100), 358.19139(25)$ \\
\hline & 20 & $214.12235(100)$ \\
\hline & 40 & $\begin{array}{l}144.04403(100), 214.12243(75), \\
43.05417(16)\end{array}$ \\
\hline \multirow{3}{*}{ JWH 018 benzimidazole analog } & 10 & $343.18056(100)$ \\
\hline & 20 & $\begin{array}{l}343.18089(100), 215.11756(46), \\
273.10216(30), 155.04888(18)\end{array}$ \\
\hline & 40 & $\begin{array}{l}\text { 155.04889(100), 127.05411(70), } \\
145.03937(30), 131.05994(16)\end{array}$ \\
\hline \multirow{3}{*}{$\begin{array}{c}\text { JWH } 018 \text { N-(1-ethylpropyl) } \\
\text { isomer }\end{array}$} & 10 & $342.18495(100)$ \\
\hline & 20 & $\begin{array}{l}\text { 155.04897(100), 342.18524(99), } \\
214.12262(12), 144.04442(11)\end{array}$ \\
\hline & 40 & $\begin{array}{l}\text { 127.05428(100), 155.04894(91), } \\
144.04412(48)\end{array}$ \\
\hline \multirow{3}{*}{$\begin{array}{l}\text { JWH } 018 \text { N-(4-oxo-pentyl) } \\
\text { metabolite }\end{array}$} & 10 & $356.16468(100), 155.04894(24)$ \\
\hline & 20 & $155.04899(100), 356.16464(17)$ \\
\hline & 40 & $\begin{array}{l}\text { 127.05414(100), 155.04895(83), } \\
85.06484(13), 43.01812(12)\end{array}$ \\
\hline \multirow{3}{*}{$\begin{array}{c}\text { JWH } 018 \text { N-(5-hydroxypentyl) } \\
\text { metabolite }\end{array}$} & 10 & $358.18031(100), 155.04876(31)$ \\
\hline & 20 & $155.04881(100), 358.18008(14)$ \\
\hline & 40 & $\begin{array}{l}\text { 127.05397(100), 155.0488(91), } \\
144.04422(12)\end{array}$ \\
\hline \multirow{3}{*}{$\begin{array}{c}\text { JWH } 018 \mathrm{~N} \text {-(5-hydroxypentyl) } \\
\beta \text {-D-Glucuronide }\end{array}$} & 10 & $\begin{array}{l}358.17854(100), 534.21023(75), \\
155.04867(21)\end{array}$ \\
\hline & 20 & $358.17916(100), 155.04793(59)$ \\
\hline & 40 & $155.04839(100), 127.05485(12)$ \\
\hline \multirow{3}{*}{$\begin{array}{c}\text { JWH } 018 \text { N-pentanoic acid } \beta \text {-D- } \\
\text { Glucuronide }\end{array}$} & 10 & $372.1595(100), 548.18965(26)$ \\
\hline & 20 & $372.15923(100), 155.04822(30)$ \\
\hline & 40 & $\begin{array}{l}\text { 155.04922(100), 372.15997(16), } \\
127.05253(12)\end{array}$ \\
\hline \multirow{3}{*}{$\begin{array}{l}\text { JWH } 018 \text { N-propanoic acid } \\
\text { metabolite }\end{array}$} & 10 & $344.12759(100)$ \\
\hline & 20 & $\begin{array}{l}\text { 155.04863(100), 344.12812(61), } \\
216.0651(25)\end{array}$ \\
\hline & 40 & $\begin{array}{l}\text { 127.05395(100), 155.04838(58), } \\
216.06493(18)\end{array}$ \\
\hline \multirow{2}{*}{$\begin{array}{l}\text { JWH } 019 \text { 5-hydroxyindole } \\
\text { metabolite }\end{array}$} & 10 & $372.19623(100)$ \\
\hline & 20 & $\begin{array}{l}372.19547(100), 155.04901(70), \\
244.13285(17)\end{array}$ \\
\hline
\end{tabular}




\begin{tabular}{|c|c|c|}
\hline & 40 & $\begin{array}{l}155.04867(100), 127.05409(99), \\
160.03885(14)\end{array}$ \\
\hline \multirow{3}{*}{$\begin{array}{l}\text { JWH } 019 \text { N-(2-fluorohexyl) } \\
\text { isomer }\end{array}$} & 10 & $374.19147(100)$ \\
\hline & 20 & $\begin{array}{l}374.19151(100), 155.04866(75), \\
246.12832(21)\end{array}$ \\
\hline & 40 & $\begin{array}{l}\text { 155.04884(100), 127.05404(97), } \\
246.12835(21), 144.04371(13)\end{array}$ \\
\hline \multirow{3}{*}{$\begin{array}{l}\text { JWH } 019 \text { N-(3-fluorohexyl) } \\
\text { isomer }\end{array}$} & 10 & $374.19142(100)$ \\
\hline & 20 & $\begin{array}{l}374.19107(100), 155.04886(70), \\
246.12872(19)\end{array}$ \\
\hline & 40 & $\begin{array}{l}155.04842(100), 127.05395(90), \\
246.12956(16), 144.04406(11)\end{array}$ \\
\hline \multirow{3}{*}{$\begin{array}{l}\text { JWH } 019 \text { N-(4-fluorohexyl) } \\
\text { isomer }\end{array}$} & 10 & $374.19147(100)$ \\
\hline & 20 & $\begin{array}{l}374.19129(100), 155.04898(78), \\
246.12871(14)\end{array}$ \\
\hline & 40 & $\begin{array}{l}\text { 155.04915(100), } 127.05429(82), \\
144.0434(13)\end{array}$ \\
\hline \multirow{3}{*}{$\begin{array}{l}\text { JWH } 019 \text { N-(5-fluorohexyl) } \\
\text { isomer }\end{array}$} & 10 & $374.19129(100)$ \\
\hline & 20 & $\begin{array}{l}\text { 374.19135(100), 155.0488(89), } \\
\text { 354.18542(17), 246.1283(14) }\end{array}$ \\
\hline & 40 & $\begin{array}{l}\text { 155.04852(100), 127.05384(86), } \\
144.04367(12)\end{array}$ \\
\hline \multirow{3}{*}{$\begin{array}{l}\text { JWH } 019 \text { N-(5-hydroxyhexyl) } \\
\text { metabolite }\end{array}$} & 10 & $372.1955(100), 155.04848(47)$ \\
\hline & 20 & $155.04868(100)$ \\
\hline & 40 & $155.0487(100), 127.05403(86)$ \\
\hline \multirow{3}{*}{$\begin{array}{l}\text { JWH } 019 \text { N-(6-fluorohexyl) } \\
\text { isomer }\end{array}$} & 10 & $374.19162(100)$ \\
\hline & 20 & $\begin{array}{l}374.19113(100), 155.04903(61), \\
246.12808(15)\end{array}$ \\
\hline & 40 & $\begin{array}{l}\text { 155.04871(100), 127.05394(87), } \\
144.04347(19), 246.12794(11)\end{array}$ \\
\hline \multirow{3}{*}{$\begin{array}{l}\text { JWH } 019 \text { N-(6-hydroxyhexyl) } \\
\text { metabolite }\end{array}$} & 10 & $372.19583(100), 155.04883(46)$ \\
\hline & 20 & $155.04886(100)$ \\
\hline & 40 & $155.04866(100), 127.0541(83)$ \\
\hline \multirow{3}{*}{$\begin{array}{c}\text { JWH } 019 \text { N-(6-hydroxyhexyl) } \\
\beta \text {-D-Glucuronide }\end{array}$} & 10 & $\begin{array}{l}372.19554(100), 548.22603(54), \\
155.04802(22)\end{array}$ \\
\hline & 20 & $372.19577(100), 155.04842(69)$ \\
\hline & 40 & $155.04837(100)$ \\
\hline \multirow{3}{*}{ JWH 030 2-naphthoyl isomer } & 10 & $292.16976(100), 155.04879(47)$ \\
\hline & 20 & $155.0489(100), 164.10682(14)$ \\
\hline & 40 & $127.05413(100), 155.04861(24)$ \\
\hline \multirow{2}{*}{ JWH 031 2'-isomer } & 10 & $306.18494(100), 155.0487(35)$ \\
\hline & 20 & $155.0488(100), 178.12241(12)$ \\
\hline
\end{tabular}




\begin{tabular}{|c|c|c|}
\hline & 40 & $127.05391(100), 155.04869(34)$ \\
\hline \multirow{3}{*}{ JWH 071} & 10 & $300.13787(100), 155.04898(11)$ \\
\hline & 20 & $\begin{array}{l}155.04872(100), 300.13813(30), \\
172.07525(25)\end{array}$ \\
\hline & 40 & $\begin{array}{l}\text { 127.05391(100), 155.04876(26), } \\
172.07504(11)\end{array}$ \\
\hline \multirow{3}{*}{$\begin{array}{l}\text { JWH } 073 \text { 4-hydroxyindole } \\
\text { metabolite }\end{array}$} & 10 & $344.16411(100)$ \\
\hline & 20 & $\begin{array}{l}\text { 155.04832(100), 344.16347(87), } \\
216.10097(21)\end{array}$ \\
\hline & 40 & $\begin{array}{l}127.05398(100), 155.04852(67), \\
160.03883(17)\end{array}$ \\
\hline \multirow{3}{*}{$\begin{array}{c}\text { JWH } 073 \text { 5-hydroxyindole } \\
\text { metabolite }\end{array}$} & 10 & $344.16411(100)$ \\
\hline & 20 & $\begin{array}{l}\text { 155.04832(100), 344.16347(87), } \\
216.10097(21)\end{array}$ \\
\hline & 40 & $\begin{array}{l}127.05398(100), 155.04852(67), \\
160.03883(17)\end{array}$ \\
\hline \multirow{3}{*}{$\begin{array}{c}\text { JWH } 073 \text { 6-hydroxyindole } \\
\text { metabolite }\end{array}$} & 10 & $344.16452(100)$ \\
\hline & 20 & $155.04885(100), 344.16474(48)$ \\
\hline & 40 & $127.05409(100), 155.04854(64)$ \\
\hline \multirow{3}{*}{$\begin{array}{c}\text { JWH } 073 \text { 6-methoxyindole } \\
\text { analog }\end{array}$} & 10 & $358.1799(100), 155.04864(10)$ \\
\hline & 20 & $155.0487(100), 358.18016(46)$ \\
\hline & 40 & $127.05391(100), 155.04858(83)$ \\
\hline \multirow{3}{*}{$\begin{array}{l}\text { JWH } 073 \text { 7-hydroxyindole } \\
\text { metabolite }\end{array}$} & 10 & $344.16439(100)$ \\
\hline & 20 & $\begin{array}{l}155.04853(100), 344.16436(94), \\
216.1013(24)\end{array}$ \\
\hline & 40 & $\begin{array}{l}\text { 127.0539(100), 155.04866(67), } \\
160.03896(24)\end{array}$ \\
\hline \multirow{3}{*}{$\begin{array}{l}\text { JWH } 073 \text { N-(2-hydroxybutyl) } \\
\text { metabolite }\end{array}$} & 10 & $344.16451(100)$ \\
\hline & 20 & $\begin{array}{l}155.04887(100), 344.16454(81), \\
216.10148(16)\end{array}$ \\
\hline & 40 & $\begin{array}{l}127.05405(100), 155.04869(72), \\
144.04405(18)\end{array}$ \\
\hline \multirow{3}{*}{$\begin{array}{l}\text { JWH } 073 \text { N-(4-hydroxybutyl) } \\
\text { metabolite }\end{array}$} & 10 & $344.1641(100), 155.04872(19)$ \\
\hline & 20 & $155.04864(100), 344.16374(24)$ \\
\hline & 40 & $\begin{array}{l}\text { 127.05388(100), 155.0486(74), } \\
144.04396(13)\end{array}$ \\
\hline \multirow{3}{*}{$\begin{array}{c}\text { JWH } 073 \text { N-(4-hydroxybutyl) } \beta \text { - } \\
\text { D-Glucuronide }\end{array}$} & 10 & $\begin{array}{l}\text { 520.19659(100), 344.16451(83), } \\
155.04914(23)\end{array}$ \\
\hline & 20 & $\begin{array}{l}344.16451(100), 155.04914(53), \\
520.19659(10)\end{array}$ \\
\hline & 40 & $155.04914(100), 127.05423(13)$ \\
\hline \multirow{2}{*}{$\begin{array}{l}\text { JWH } 073 \text { N-butanoic acid } \\
\text { metabolite }\end{array}$} & 10 & $358.14334(100), 155.04805(13)$ \\
\hline & 20 & $155.04888(100), 358.14371(44)$ \\
\hline
\end{tabular}




\begin{tabular}{|c|c|c|}
\hline & 40 & $\begin{array}{l}127.05405(100), 155.04903(79), \\
144.04354(13)\end{array}$ \\
\hline \multirow{3}{*}{$\begin{array}{c}( \pm)-J W H 073 \mathrm{~N}-(3- \\
\text { hydroxybutyl) metabolite }\end{array}$} & 10 & $344.16438(100), 155.04816(14)$ \\
\hline & 20 & $155.04863(100), 344.16415(37)$ \\
\hline & 40 & $127.05398(100), 155.04881(74)$ \\
\hline \multirow{3}{*}{$\begin{array}{l}\text { (R)-(-)-JWH } 073 \mathrm{~N}-(3- \\
\text { hydroxybutyl) metabolite }\end{array}$} & 10 & $344.16432(100), 155.04874(14)$ \\
\hline & 20 & $155.04872(100), 344.16417(37)$ \\
\hline & 40 & $127.05389(100), 155.04855(73)$ \\
\hline \multirow{3}{*}{$\begin{array}{l}\text { (S)-(+)-JWH } 073 \mathrm{~N}-(3- \\
\text { hydroxybutyl) metabolite }\end{array}$} & 10 & $344.16432(100), 155.04874(14)$ \\
\hline & 20 & 155.04872(100), 344.16417(37) \\
\hline & 40 & $127.05389(100), 155.04855(73)$ \\
\hline \multirow{3}{*}{ JWH 080} & 10 & $358.18025(100)$ \\
\hline & 20 & $\begin{array}{l}185.05942(100), 358.18007(76), \\
200.10638(33)\end{array}$ \\
\hline & 40 & $\begin{array}{l}\text { 185.0594(100), 157.06449(59), } \\
144.04404(29), 200.10645(20), \\
127.05422(13)\end{array}$ \\
\hline \multirow{3}{*}{$\begin{array}{c}\text { JWH } 081 \text { 4-hydroxynaphthyl } \\
\text { metabolite }\end{array}$} & 10 & $358.17969(100)$ \\
\hline & 20 & $\begin{array}{l}\text { 171.04385(100), 358.17981(90), } \\
214.12166(23)\end{array}$ \\
\hline & 40 & $\begin{array}{l}171.04365(100), 143.04898(43), \\
115.05384(17), 144.04501(17)\end{array}$ \\
\hline \multirow{3}{*}{$\begin{array}{l}\text { JWH } 081 \text { N-(4-hydroxypentyl) } \\
\text { metabolite }\end{array}$} & 10 & $388.19072(100), 185.05924(19)$ \\
\hline & 20 & $185.05945(100), 388.19091(35)$ \\
\hline & 40 & $\begin{array}{l}\text { 185.05943(100), 157.06412(36), } \\
144.04417(12)\end{array}$ \\
\hline \multirow{3}{*}{$\begin{array}{l}\text { JWH } 081 \text { N-(5-hydroxypentyl) } \\
\text { metabolite }\end{array}$} & 10 & \\
\hline & 20 & $185.05941(100), 388.19067(28)$ \\
\hline & 40 & $\begin{array}{l}\text { 185.05947(100), 157.06447(37), } \\
144.04444(15)\end{array}$ \\
\hline \multirow{3}{*}{$\begin{array}{l}\text { JWH } 081 \text { N-pentanoic acid } \\
\text { metabolite }\end{array}$} & 10 & $402.17045(100), 185.05877(13)$ \\
\hline & 20 & $\begin{array}{l}\text { 185.05911(100), 402.17062(70), } \\
244.09684(15)\end{array}$ \\
\hline & 40 & $\begin{array}{l}\text { 185.05907(100), } 157.06425(34), \\
144.04445(15)\end{array}$ \\
\hline \multirow{3}{*}{$\begin{array}{l}\text { JWH 081-N-(cyclohexylmethyl) } \\
\text { analog }\end{array}$} & 10 & $398.21153(100)$ \\
\hline & 20 & $\begin{array}{l}398.21162(100), 185.05946(46), \\
240.13795(14)\end{array}$ \\
\hline & 40 & $\begin{array}{l}185.05949(100), 157.06436(25), \\
144.04405(18)\end{array}$ \\
\hline JWH 116 & 10 & $370.21647(100)$ \\
\hline
\end{tabular}




\begin{tabular}{|c|c|c|}
\hline & 20 & $\begin{array}{l}370.21645(100), 183.08009(55), \\
214.12216(42)\end{array}$ \\
\hline & 40 & $\begin{array}{l}\text { 144.04422(100), 155.08497(84), } \\
183.07978(81), 214.12217(64), \\
141.06957(51)\end{array}$ \\
\hline \multirow{3}{*}{$\begin{array}{l}\text { JWH } 122 \text { N-(4-hydroxypentyl) } \\
\text { metabolite }\end{array}$} & 10 & $372.1955(100), 155.04848(47)$ \\
\hline & 20 & $155.04868(100)$ \\
\hline & 40 & $155.0487(100), 127.05403(86)$ \\
\hline \multirow{3}{*}{$\begin{array}{l}\text { JWH } 122 \mathrm{~N}-(5 \text {-hydroxypentyl) } \\
\text { metabolite }\end{array}$} & 10 & $372.19587(100), 169.06461(27)$ \\
\hline & 20 & $169.06454(100), 372.19622(22)$ \\
\hline & 40 & $\begin{array}{l}\text { 169.06471(100), 141.06976(83), } \\
144.04373(17)\end{array}$ \\
\hline \multirow{3}{*}{ JWH 145 2-phenyl isomer } & 10 & $368.20084(100), 155.04879(28)$ \\
\hline & 20 & $155.04883(100), 368.20129(11)$ \\
\hline & 40 & $127.05407(100), 155.04899(90)$ \\
\hline \multirow{3}{*}{ JWH 146} & 10 & $396.23189(100), 155.04867(40)$ \\
\hline & 20 & $155.04889(100)$ \\
\hline & 40 & $155.04887(100), 127.05399(80)$ \\
\hline \multirow{3}{*}{ JWH 149} & 10 & $370.21591(100)$ \\
\hline & 20 & $\begin{array}{l}370.21622(100), 169.06418(97), \\
228.13783(22)\end{array}$ \\
\hline & 40 & $\begin{array}{l}\text { 169.06417(100), 141.06956(87), } \\
158.05945(19), 228.13735(14)\end{array}$ \\
\hline \multirow{3}{*}{ JWH 167} & 10 & $306.18524(100)$ \\
\hline & 20 & $\begin{array}{l}\text { 91.05412(100), 306.18506(95), } \\
214.12257(64), 188.14263(26)\end{array}$ \\
\hline & 40 & $\begin{array}{l}\text { 91.05425(100), 144.04423(50), } \\
214.12246(19)\end{array}$ \\
\hline \multirow{3}{*}{ JWH 176} & 10 & $\begin{array}{l}255.11683(100), 325.19508(86), \\
141.06988(11), 324.18725(11)\end{array}$ \\
\hline & 20 & $\begin{array}{l}\text { 255.11683(100), 141.06988(24), } \\
253.10118(18), 254.109(13)\end{array}$ \\
\hline & 40 & \\
\hline \multirow{3}{*}{ JWH 193} & 10 & $399.20638(100), 169.0642(18)$ \\
\hline & 20 & $\begin{array}{l}\text { 169.06439(100), 399.2067(38), } \\
\text { 114.09134(31) }\end{array}$ \\
\hline & 40 & $\begin{array}{l}\text { 169.0643(100), 114.09129(75), } \\
141.06936(52), 70.06525(12)\end{array}$ \\
\hline \multirow[b]{2}{*}{ JWH 198} & 10 & $415.20157(100), 185.05911(19)$ \\
\hline & 20 & $\begin{array}{l}185.05938(100), 415.20162(41), \\
114.09124(25)\end{array}$ \\
\hline
\end{tabular}




\begin{tabular}{|c|c|c|}
\hline & 40 & $\begin{array}{l}185.05946(100), 114.09142(55), \\
157.06473(23)\end{array}$ \\
\hline \multirow{3}{*}{$\begin{array}{l}\text { JWH } 200 \text { 4-hydroxyindole } \\
\text { metabolite }\end{array}$} & 10 & 401.1854(100) \\
\hline & 20 & $\begin{array}{l}\text { 155.04869(100), 401.18597(90), } \\
114.09131(74)\end{array}$ \\
\hline & 40 & $\begin{array}{l}114.09126(100), 155.04888(64), \\
127.05435(34), 70.06478(15)\end{array}$ \\
\hline \multirow{3}{*}{$\begin{array}{l}\text { JWH } 200 \text { 5-hydroxyindole } \\
\text { metabolite }\end{array}$} & 10 & $401.18602(100), 155.04887(15)$ \\
\hline & 20 & $\begin{array}{l}\text { 155.04907(100), 114.09171(30), } \\
401.18662(29)\end{array}$ \\
\hline & 40 & $\begin{array}{l}155.04905(100), 114.09131(94), \\
127.05448(58), 70.06499(16)\end{array}$ \\
\hline \multirow{3}{*}{$\begin{array}{l}\text { JWH } 200 \text { 6-hydroxyindole } \\
\text { metabolite }\end{array}$} & 10 & $401.18588(100), 155.04891(24)$ \\
\hline & 20 & $\begin{array}{l}\text { 155.04921(100), 401.18635(24), } \\
114.09095(21)\end{array}$ \\
\hline & 40 & $\begin{array}{l}155.04876(100), 127.05407(70), \\
114.09171(50), 70.06396(11)\end{array}$ \\
\hline \multirow{3}{*}{$\begin{array}{l}\text { JWH } 200 \text { 7-hydroxyindole } \\
\text { metabolite }\end{array}$} & 10 & $401.18597(100)$ \\
\hline & 20 & $\begin{array}{l}\text { 155.04842(100), 401.18571(90), } \\
114.0911(77)\end{array}$ \\
\hline & 40 & $\begin{array}{l}\text { 114.09121(100), 155.04868(66), } \\
127.05338(34), 70.06431(17)\end{array}$ \\
\hline \multirow{3}{*}{ JWH 203} & 10 & $340.14619(100)$ \\
\hline & 20 & $\begin{array}{l}340.14596(100), 125.01508(90) \\
188.14249(23), 214.12275(11)\end{array}$ \\
\hline & 40 & $125.01519(100), 144.04449(10)$ \\
\hline \multirow{3}{*}{$\begin{array}{c}\text { JWH } 203 \mathrm{~N} \text {-(4-hydroxypentyl) } \\
\text { metabolite }\end{array}$} & 10 & $356.14118(100), 186.12737(26)$ \\
\hline & 20 & $\begin{array}{l}\text { 125.01534(100), 186.12747(84), } \\
356.14075(19), 130.06503(16), \\
282.0681(10)\end{array}$ \\
\hline & 40 & $125.01529(100)$ \\
\hline \multirow{3}{*}{$\begin{array}{c}\text { JWH } 203 \mathrm{~N} \text {-(5-hydroxypentyl) } \\
\text { metabolite }\end{array}$} & 10 & $356.14058(100), 186.12701(11)$ \\
\hline & 20 & $\begin{array}{l}125.01503(100), 186.12714(36) \\
204.13768(30), 356.1395(18)\end{array}$ \\
\hline & 40 & $125.01507(100)$ \\
\hline \multirow{3}{*}{$\begin{array}{l}\text { JWH } 203 \mathrm{~N} \text {-pentanoic acid } \\
\text { metabolite }\end{array}$} & 10 & $370.12015(100), 200.10631(12)$ \\
\hline & 20 & $\begin{array}{l}125.01478(100), 200.10624(54), \\
370.12045(37), 218.11713(24)\end{array}$ \\
\hline & 40 & $125.01491(100)$ \\
\hline \multirow[b]{2}{*}{$\begin{array}{l}\text { JWH } 210 \text { 2-ethylnaphthyl } \\
\text { isomer }\end{array}$} & 10 & $370.21647(100)$ \\
\hline & 20 & $\begin{array}{l}370.21645(100), 183.08009(55), \\
214.12216(42)\end{array}$ \\
\hline
\end{tabular}




\begin{tabular}{|c|c|c|}
\hline & 40 & $\begin{array}{l}144.04422(100), 155.08497(84), \\
183.07978(81), 214.12217(64), \\
141.06957(51)\end{array}$ \\
\hline \multirow{3}{*}{$\begin{array}{c}\text { JWH } 210 \text { 5-hydroxyindole } \\
\text { metabolite }\end{array}$} & 10 & $386.21146(100)$ \\
\hline & 20 & $\begin{array}{l}386.21164(100), 183.0799(62), \\
230.11634(17)\end{array}$ \\
\hline & 40 & $\begin{array}{l}\text { 183.08055(100), 155.08506(50), } \\
230.11714(24), 160.03803(23), \\
153.06916(20)\end{array}$ \\
\hline \multirow{3}{*}{$\begin{array}{c}\text { JWH } 210 \text { N-(4-hydroxypentyl) } \\
\text { metabolite }\end{array}$} & 10 & $386.21146(100), 183.07986(16)$ \\
\hline & 20 & 183.07995(100), 386.21091(39) \\
\hline & 40 & $\begin{array}{l}183.07987(100), 155.08505(45), \\
153.06853(18), 144.0433(15)\end{array}$ \\
\hline \multirow{3}{*}{$\begin{array}{c}\text { JWH } 210 \mathrm{~N} \text {-(5-hydroxypentyl) } \\
\text { metabolite }\end{array}$} & 10 & $386.21122(100), 183.08002(23)$ \\
\hline & 20 & 183.08012(100), 386.21097(28) \\
\hline & 40 & $\begin{array}{l}\text { 183.0799(100), 155.08475(48), } \\
153.06952(19), 144.04371(17)\end{array}$ \\
\hline \multirow{3}{*}{$\begin{array}{c}\text { JWH } 210 \mathrm{~N} \text {-pentanoic acid } \\
\text { metabolite }\end{array}$} & 10 & $400.19072(100), 183.08025(12)$ \\
\hline & 20 & $\begin{array}{l}\text { 183.08044(100), 400.1908(73), } \\
244.09647(13)\end{array}$ \\
\hline & 40 & $\begin{array}{l}183.07985(100), 155.0847(43), \\
144.04433(16), 153.06926(16)\end{array}$ \\
\hline \multirow{3}{*}{ JWH 213} & 10 & $384.23191(100)$ \\
\hline & 20 & $\begin{array}{l}384.23188(100), 183.08006(79), \\
228.13773(21)\end{array}$ \\
\hline & 40 & $\begin{array}{l}\text { 183.07984(100), 155.08492(47), } \\
153.06982(20), 158.05948(19), \\
228.13742(19)\end{array}$ \\
\hline \multirow{3}{*}{$\begin{array}{c}\text { JWH } 250 \text { 5-hydroxyindole } \\
\text { metabolite }\end{array}$} & 10 & \\
\hline & 20 & $121.06479(100), 352.19072(18)$ \\
\hline & 40 & $\begin{array}{l}\text { 91.05423(100), 121.06479(82), } \\
93.06988(24), 146.06004(14), \\
160.07569(14), 131.04914(11)\end{array}$ \\
\hline \multirow{3}{*}{$\begin{array}{c}\text { JWH } 250 \mathrm{~N} \text {-(4-hydroxypentyl) } \\
\text { metabolite }\end{array}$} & 10 & $\begin{array}{l}352.19072(100), 121.06479(14), \\
186.12773(12)\end{array}$ \\
\hline & 20 & $\begin{array}{l}\text { 121.06479(100), 186.12773(18), } \\
352.19072(14)\end{array}$ \\
\hline & 40 & $\begin{array}{l}91.05423(100), 121.06479(89), \\
93.06988(26), 130.06513(20), \\
69.06988(12)\end{array}$ \\
\hline \multirow{2}{*}{$\begin{array}{l}\text { JWH } 250 \text { N-(5-hydroxypentyl) } \\
\text { metabolite }\end{array}$} & 10 & $352.19072(100), 121.06479(20)$ \\
\hline & 20 & $121.06479(100)$ \\
\hline
\end{tabular}




\begin{tabular}{|c|c|c|}
\hline & 40 & $\begin{array}{l}91.05423(100), 121.06479(87), \\
93.06988(25), 130.06513(20)\end{array}$ \\
\hline \multirow{3}{*}{$\begin{array}{l}\text { JWH } 250 \text { N-pentanoic acid } \\
\text { metabolite }\end{array}$} & 10 & $366.16998(100), 121.06479(17)$ \\
\hline & 20 & 121.06479(100), 366.16998(18) \\
\hline & 40 & $\begin{array}{l}121.06479(100), 91.05423(92), \\
93.06988(24), 130.06513(18), \\
55.05423(12)\end{array}$ \\
\hline \multirow{3}{*}{ JWH 309 5'-isomer } & 10 & $418.21654(100), 155.04914(23)$ \\
\hline & 20 & $155.04914(100), 418.21654(27)$ \\
\hline & 40 & $155.04914(100), 127.05423(64)$ \\
\hline \multirow{3}{*}{ JWH 387} & 10 & $420.09575(100)$ \\
\hline & 20 & $\begin{array}{l}420.09575(100), 232.95965(90), \\
214.12264(10)\end{array}$ \\
\hline & 40 & $\begin{array}{l}232.95965(100), 204.96474(69), \\
126.0464(12)\end{array}$ \\
\hline \multirow{3}{*}{$\begin{array}{c}\text { JWH } 398 \text { N-(4-hydroxypentyl) } \\
\text { metabolite }\end{array}$} & 10 & $392.14118(100), 189.01017(24)$ \\
\hline & 20 & $189.01017(100), 392.14118(22)$ \\
\hline & 40 & $189.01017(100), 161.01525(58)$ \\
\hline \multirow{3}{*}{$\begin{array}{c}\text { JWH } 398 \text { N-(5-hydroxypentyl) } \\
\text { metabolite }\end{array}$} & 10 & $392.14118(100), 189.01017(36)$ \\
\hline & 20 & $189.01017(100), 392.14118(15)$ \\
\hline & 40 & $189.01017(100), 161.01525(55)$ \\
\hline \multirow{3}{*}{$\begin{array}{c}\text { JWH } 398 \text { N-pentanoic acid } \\
\text { metabolite }\end{array}$} & 10 & $406.12045(100), 189.01017(18)$ \\
\hline & 20 & $189.01017(100), 406.12045(30)$ \\
\hline & 40 & $189.01017(100), 161.01525(51)$ \\
\hline \multirow{3}{*}{ JWH 412} & 10 & $360.17582(100)$ \\
\hline & 20 & $173.03972(100), 360.17582(85)$ \\
\hline & 40 & $173.03972(100), 145.0448(67)$ \\
\hline \multirow[t]{3}{*}{$\begin{array}{l}\text { CP 47,497-C } 8 \text {-homolog C-8- } \\
\text { hydroxy metabolite }\end{array}$} & 10 & $\begin{array}{l}\text { 175.11174(100), 331.26316, (70), } \\
83.08553(37), 157.15869(25), \\
81.06988(11), 251.20056(11), \\
97.10118(11)\end{array}$ \\
\hline & 20 & \\
\hline & 40 & \\
\hline \multirow{3}{*}{$\begin{array}{l}\text { RCS-4 4-hydroxyphenyl } \\
\text { metabolite }\end{array}$} & 10 & $308.16451(100), 121.02841(12)$ \\
\hline & 20 & 121.02841(100), 308.16451(30) \\
\hline & 40 & $\begin{array}{l}\text { 121.02841(100), 93.03349(37), } \\
65.03858(17)\end{array}$ \\
\hline \multirow{3}{*}{ RCS-4 M10 metabolite } & 10 & $324.15942(100), 121.02841(54)$ \\
\hline & 20 & $121.02841(100)$ \\
\hline & 40 & $\begin{array}{l}\text { 121.02841(100), 93.03349(30), } \\
65.03858(14)\end{array}$ \\
\hline RCS-4 M11 metabolite & 10 & $322.14377(100), 121.02841(47)$ \\
\hline
\end{tabular}




\begin{tabular}{|c|c|c|}
\hline & 20 & $121.02841(100)$ \\
\hline & 40 & $\begin{array}{l}\text { 121.02841(100), 93.03349(34), } \\
65.03858(13)\end{array}$ \\
\hline \multirow{3}{*}{ RCS-4 M9 metabolite } & 10 & $\begin{array}{l}324.15942(100), 121.02841(36), \\
186.12773(11)\end{array}$ \\
\hline & 20 & $121.02841(100)$ \\
\hline & 40 & $\begin{array}{l}\text { 121.02841(100), 93.03349(29), } \\
65.03858(12)\end{array}$ \\
\hline \multirow{3}{*}{$\begin{array}{l}\text { RCS-4 N-(4-hydroxypentyl) } \\
\text { metabolite }\end{array}$} & 10 & $338.17507(100), 135.04406(36)$ \\
\hline & 20 & $135.04406(100)$ \\
\hline & 40 & $\begin{array}{l}\text { 135.04406(100), 77.03858(23), } \\
107.04914(17)\end{array}$ \\
\hline \multirow{3}{*}{$\begin{array}{l}\text { RCS-4 N-(5-carboxypentyl) } \\
\text { metabolite }\end{array}$} & 10 & $352.15433(100), 135.04406(22)$ \\
\hline & 20 & $135.04406(100), 352.15433(21)$ \\
\hline & 40 & $\begin{array}{l}\text { 135.04406(100), 77.03858(18), } \\
\text { 107.04914(16) }\end{array}$ \\
\hline \multirow{3}{*}{$\begin{array}{l}\text { RCS-4 N-(5-hydroxypentyl) } \\
\text { metabolite }\end{array}$} & 10 & $338.17507(100), 135.04406(55)$ \\
\hline & 20 & \\
\hline & 40 & \\
\hline \multirow[b]{3}{*}{ 5-fluoro NPB-22 } & 10 & $233.10836(100), 378.1612(80)$ \\
\hline & 20 & $233.10814(100), 213.10145(21)$ \\
\hline & 40 & $\begin{array}{l}\text { 145.03926(100), 213.10242(33), } \\
\text { 177.04546(21), 69.06945(20), } \\
41.03849(10)\end{array}$ \\
\hline \multirow{3}{*}{ 5-fluoro PB-22 } & 10 & $232.11323(100)$ \\
\hline & 20 & $232.11315(100)$ \\
\hline & 40 & $144.04429(100), 232.11317(90)$ \\
\hline \multirow{3}{*}{$\begin{array}{c}\text { 5-fluoro PB-22 3-carboxyindole } \\
\text { metabolite }\end{array}$} & 10 & $\begin{array}{l}250.12378(100), 206.13395(40), \\
118.06513(11)\end{array}$ \\
\hline & 20 & $\begin{array}{l}\text { 118.06513(100), 132.08078(59), } \\
206.13395(57), \\
\text { 174.05495(27),130.06513(24), } \\
\text { 232.11322(18), 69.06988(13), } \\
41.03858(11)\end{array}$ \\
\hline & 40 & \\
\hline \multirow{3}{*}{$\begin{array}{l}\text { 5-fluoro PB-22 3- } \\
\text { hydroxyquinoline isomer }\end{array}$} & 10 & $377.16596(100), 232.11309(34)$ \\
\hline & 20 & $232.11315(100)$ \\
\hline & 40 & $232.11304(100), 144.04431(90)$ \\
\hline \multirow{4}{*}{$\begin{array}{l}\text { 5-fluoro PB-22 4- } \\
\text { hydroxyisoquinoline isomer }\end{array}$} & 10 & $377.16711(100), 232.11374(67)$ \\
\hline & 20 & $232.11318(100)$ \\
\hline & 40 & $232.1138(100), 144.04445(95)$ \\
\hline & 10 & $232.11243(100)$ \\
\hline
\end{tabular}




\begin{tabular}{|c|c|c|}
\hline \multirow{2}{*}{$\begin{array}{c}\text { 5-fluoro PB-22 4- } \\
\text { hydroxyquinoline isomer }\end{array}$} & 20 & $232.11232(100)$ \\
\hline & 40 & $144.04412(100), 232.11246(76)$ \\
\hline \multirow{3}{*}{$\begin{array}{l}\text { 5-fluoro PB-22 5- } \\
\text { hydroxyquinoline isomer }\end{array}$} & 10 & $377.16595(100), 232.1125(11)$ \\
\hline & 20 & $232.11321(100), 377.16592(43)$ \\
\hline & 40 & $232.11289(100), 144.04419(85)$ \\
\hline \multirow{3}{*}{$\begin{array}{l}\text { 5-fluoro PB-22 6- } \\
\text { hydroxyisoquinoline isomer }\end{array}$} & 10 & $377.16711(100), 232.11374(67)$ \\
\hline & 20 & $232.11318(100)$ \\
\hline & 40 & $232.1138(100), 144.04445(95)$ \\
\hline \multirow{3}{*}{$\begin{array}{l}\text { 5-fluoro PB-22 6- } \\
\text { hydroxyquinoline isomer }\end{array}$} & 10 & $377.16595(100), 232.1125(11)$ \\
\hline & 20 & $232.11321(100), 377.16592(43)$ \\
\hline & 40 & $232.11289(100), 144.04419(85)$ \\
\hline \multirow{3}{*}{$\begin{array}{l}\text { 5-fluoro PB-22 7- } \\
\text { hydroxyisoquinoline isomer }\end{array}$} & 10 & $377.16698(100), 232.11358(51)$ \\
\hline & 20 & $232.11358(100)$ \\
\hline & 40 & $232.11372(100), 144.0445(86)$ \\
\hline \multirow{3}{*}{$\begin{array}{l}\text { 5-fluoro PB-22 7- } \\
\text { hydroxyquinoline isomer }\end{array}$} & 10 & $232.11329(100)$ \\
\hline & 20 & $232.11319(100)$ \\
\hline & 40 & $\begin{array}{l}144.04415(100), 232.11325(96), \\
212.10664(33), 69.06999(11)\end{array}$ \\
\hline \multirow{3}{*}{$\begin{array}{l}\text { 5-fluoro PB-22 8- } \\
\text { hydroxyisoquinoline isomer }\end{array}$} & 10 & $377.16698(100), 232.11358(51)$ \\
\hline & 20 & $232.11358(100)$ \\
\hline & 40 & $232.11372(100), 144.0445(86)$ \\
\hline \multirow{3}{*}{$\begin{array}{l}\text { 5-fluoro PB-22 N-(2- } \\
\text { fluoropentyl) isomer }\end{array}$} & 10 & $232.11352(100)$ \\
\hline & 20 & $232.11323(100)$ \\
\hline & 40 & $\begin{array}{l}232.1137(100), 144.04443(47), \\
212.10724(14)\end{array}$ \\
\hline \multirow{3}{*}{$\begin{array}{l}\text { 5-fluoro PB-22 N-(3- } \\
\text { fluoropentyl) isomer }\end{array}$} & 10 & $232.11355(100)$ \\
\hline & 20 & $232.1136(100)$ \\
\hline & 40 & $\begin{array}{l}232.11376(100), 144.04443(51), \\
212.10733(25), 69.06988(14)\end{array}$ \\
\hline \multirow{3}{*}{$\begin{array}{l}\text { 5-fluoro PB-22 N-(4- } \\
\text { fluoropentyl) isomer }\end{array}$} & 10 & $232.11329(100)$ \\
\hline & 20 & $232.11319(100)$ \\
\hline & 40 & $\begin{array}{l}144.04415(100), 232.11325(96) \\
212.10664(33), 69.06999(11)\end{array}$ \\
\hline \multirow{3}{*}{ FDU-PB-22 } & 10 & $252.08152(100)$ \\
\hline & 20 & $252.08145(100), 109.04474(30)$ \\
\hline & 40 & $109.04453(100)$ \\
\hline \multirow{3}{*}{ FUB-PB-22 } & 10 & $252.08213(100)$ \\
\hline & 20 & $252.08184(100), 109.04477(28)$ \\
\hline & 40 & $109.0448(100)$ \\
\hline NPB-22 & 10 & $215.11752(100), 360.17002(38)$ \\
\hline
\end{tabular}




\begin{tabular}{|c|c|c|}
\hline & 20 & $215.11747(100)$ \\
\hline & 40 & $145.03919(100), 215.11746(21)$ \\
\hline \multirow{3}{*}{ PB-22 } & 10 & $359.17519(100), 214.12217(12)$ \\
\hline & 20 & $214.12233(100), 359.17484(29)$ \\
\hline & 40 & $\begin{array}{l}144.04408(100), 214.12205(96), \\
43.05453(13)\end{array}$ \\
\hline \multirow{3}{*}{$\begin{array}{l}\text { PB-22 3-carboxyindole } \\
\text { metabolite }\end{array}$} & 10 & $\begin{array}{l}232.13321(100), 188.14338(53), \\
132.08078(28), 118.06513(20), \\
231.12538(11)\end{array}$ \\
\hline & 20 & $\begin{array}{l}\text { 118.06513(100), 132.08078(81), } \\
\text { 43.05423(37), 174.05495(29), } \\
\text { 188.14338(25), 214.12264(16), } \\
130.06513(13), 144.04439(13)\end{array}$ \\
\hline & 40 & \\
\hline \multirow{3}{*}{$\begin{array}{l}\text { PB-22 3-hydroxyquinoline } \\
\text { isomer }\end{array}$} & 10 & $359.17547(100), 214.12239(36)$ \\
\hline & 20 & $214.12241(100)$ \\
\hline & 40 & $\begin{array}{l}\text { 144.04418(100), 214.1223(80), } \\
43.0542(14)\end{array}$ \\
\hline \multirow{3}{*}{$\begin{array}{l}\text { PB-22 4-hydroxyisoquinoline } \\
\text { isomer }\end{array}$} & 10 & $214.1223(100), 359.17495(81)$ \\
\hline & 20 & $214.12248(100)$ \\
\hline & 40 & $\begin{array}{l}\text { 144.04412(100), 214.1222(73), } \\
43.05459(14)\end{array}$ \\
\hline \multirow{3}{*}{$\begin{array}{l}\text { PB-22 5-hydroxyisoquinoline } \\
\text { isomer }\end{array}$} & 10 & $359.17519(100), 214.12217(12)$ \\
\hline & 20 & $214.12233(100), 359.17484(29)$ \\
\hline & 40 & $\begin{array}{l}\text { 144.04408(100), 214.12205(96), } \\
43.05453(13)\end{array}$ \\
\hline \multirow{3}{*}{$\begin{array}{l}\text { PB-22 5-hydroxyquinoline } \\
\text { isomer }\end{array}$} & 10 & $359.1754(100), 214.12227(61)$ \\
\hline & 20 & $214.12233(100)$ \\
\hline & 40 & $\begin{array}{l}144.04407(100), 214.12229(77), \\
43.05435(15)\end{array}$ \\
\hline \multirow{3}{*}{$\begin{array}{l}\text { PB-22 6-hydroxyisoquinoline } \\
\text { isomer }\end{array}$} & 10 & $359.17556(100), 214.12238(85)$ \\
\hline & 20 & $214.12205(100)$ \\
\hline & 40 & $\begin{array}{l}144.04423(100), 214.12237(77), \\
43.05439(16)\end{array}$ \\
\hline \multirow{3}{*}{$\begin{array}{l}\text { PB-22 6-hydroxyquinoline } \\
\text { isomer }\end{array}$} & 10 & $359.17514(100), 214.12194(12)$ \\
\hline & 20 & $214.12224(100), 359.17481(30)$ \\
\hline & 40 & $\begin{array}{l}\text { 144.04403(100), 214.12223(90), } \\
43.05439(12)\end{array}$ \\
\hline \multirow{3}{*}{$\begin{array}{c}\text { PB-22 7-hydroxyisoquinoline } \\
\text { isomer }\end{array}$} & 10 & $359.17523(100), 214.12192(10)$ \\
\hline & 20 & $214.12241(100), 359.17512(39)$ \\
\hline & 40 & $\begin{array}{l}\text { 144.04412(100), 214.12234(90), } \\
43.05456(13)\end{array}$ \\
\hline
\end{tabular}




\begin{tabular}{|c|c|c|}
\hline \multirow{3}{*}{$\begin{array}{c}\text { PB-22 7-hydroxyquinoline } \\
\text { isomer }\end{array}$} & 10 & $359.17532(100), 214.12257(97)$ \\
\hline & 20 & 214.12234(100) \\
\hline & 40 & $\begin{array}{l}144.04429(100), 214.12219(79), \\
43.05455(14)\end{array}$ \\
\hline \multirow{3}{*}{$\begin{array}{c}\text { PB-22 8-hydroxyisoquinoline } \\
\text { isomer }\end{array}$} & 10 & $359.17532(100), 214.12257(97)$ \\
\hline & 20 & $214.12234(100)$ \\
\hline & 40 & $\begin{array}{l}144.04429(100), 214.12219(79), \\
43.05455(14)\end{array}$ \\
\hline \multirow{3}{*}{$\begin{array}{l}\text { PB-22 N-(4-hydroxypentyl) } \\
\text { metabolite }\end{array}$} & 10 & $230.1175(100)$ \\
\hline & 20 & $230.11738(100), 144.04415(12)$ \\
\hline & 40 & $\begin{array}{l}\text { 144.0441(100), 69.06993(47), } \\
230.11744(15)\end{array}$ \\
\hline \multirow{3}{*}{$\begin{array}{l}\text { PB-22 N-(5-hydroxypentyl) } \\
\text { metabolite }\end{array}$} & 10 & $230.11732(100)$ \\
\hline & 20 & $230.11733(100)$ \\
\hline & 40 & $\begin{array}{l}\text { 144.04419(100), 230.11707(52), } \\
69.06979(21)\end{array}$ \\
\hline \multirow{3}{*}{$\begin{array}{l}\text { PB-22 N-pentanoic acid } \\
\text { metabolite }\end{array}$} & 10 & $244.09659(100)$ \\
\hline & 20 & $244.09686(100)$ \\
\hline & 40 & $\begin{array}{l}\text { 144.04411(100), 244.09588(40), } \\
55.05422(38), 101.05968(19), \\
83.04914(17)\end{array}$ \\
\hline \multirow{3}{*}{$\begin{array}{l}\text { PB-22 N-pentanoic acid-3- } \\
\text { carboxyindole metabolite }\end{array}$} & 10 & $244.09682(100), 200.10699(17)$ \\
\hline & 20 & $\begin{array}{l}244.09682(100), 144.04439(47), \\
200.10699(34), 172.11208(32), \\
156.08078(26), 55.05423(21), \\
101.05971(21), 83.04914(14), \\
118.06513(13)\end{array}$ \\
\hline & 40 & \\
\hline \multirow{3}{*}{$\begin{array}{l}\text { AM2201 8-quinolinyl } \\
\text { carboxamide }\end{array}$} & 10 & $232.11281(100), 376.18197(34)$ \\
\hline & 20 & $232.11268(100)$ \\
\hline & 40 & $232.11298(100), 144.04378(97)$ \\
\hline \multirow{3}{*}{ BB-22 } & 10 & $240.13795(100)$ \\
\hline & 20 & $240.13811(100)$ \\
\hline & 40 & $\begin{array}{l}\text { 144.04399(100), 240.13784(68), } \\
55.05441(33), 97.10104(11)\end{array}$ \\
\hline \multirow{4}{*}{$\begin{array}{l}\text { BB-22 3-carboxyindole } \\
\text { metabolite }\end{array}$} & 10 & $258.1487(100), 214.15839(12)$ \\
\hline & 20 & $\begin{array}{l}\text { 118.06476(100), 55.05426(74), } \\
\text { 132.08022(51), 176.07002(47), } \\
\text { 214.1601(32) }\end{array}$ \\
\hline & 40 & $\begin{array}{l}\text { 55.05452(100), 118.06426(41), } \\
91.0538(19)\end{array}$ \\
\hline & 10 & $385.19081(100), 240.13766(23)$ \\
\hline
\end{tabular}




\begin{tabular}{|c|c|c|}
\hline \multirow{2}{*}{$\begin{array}{l}\text { BB-22 3-hydroxyquinoline } \\
\text { isomer }\end{array}$} & 20 & $240.13806(100), 385.19072(15)$ \\
\hline & 40 & \\
\hline \multirow{3}{*}{$\begin{array}{l}\text { BB-22 4-hydroxyisoquinoline } \\
\text { isomer }\end{array}$} & 10 & $385.19061(100), 240.13759(71)$ \\
\hline & 20 & $240.13787(100)$ \\
\hline & 40 & $\begin{array}{l}\text { 144.0439(100), 240.13754(83), } \\
55.05427(33), 97.10078(13)\end{array}$ \\
\hline \multirow{3}{*}{$\begin{array}{l}\text { BB-22 5-hydroxyisoquinoline } \\
\text { isomer }\end{array}$} & 10 & $385.19022(100)$ \\
\hline & 20 & $240.13758(100), 385.19022(73)$ \\
\hline & 40 & $\begin{array}{l}\text { 144.04375(100), 240.13759(95), } \\
55.05409(30), 97.10075(11)\end{array}$ \\
\hline \multirow{3}{*}{$\begin{array}{l}\text { BB-22 5-hydroxyquinoline } \\
\text { isomer }\end{array}$} & 10 & $385.19058(100), 240.13757(57)$ \\
\hline & 20 & $240.13766(100)$ \\
\hline & 40 & $\begin{array}{l}144.04389(100), 240.13771(84), \\
55.05412(33), 97.10106(11)\end{array}$ \\
\hline \multirow{3}{*}{$\begin{array}{c}\text { BB-22 6-hydroxyisoquinoline } \\
\text { isomer }\end{array}$} & 10 & $385.19059(100), 240.13772(47)$ \\
\hline & 20 & $240.13712(100)$ \\
\hline & 40 & $\begin{array}{l}\text { 144.04395(100), 240.13749(84), } \\
55.05407(31), 97.10096(11)\end{array}$ \\
\hline \multirow{3}{*}{$\begin{array}{l}\text { BB-22 6-hydroxyquinoline } \\
\text { isomer }\end{array}$} & 10 & $385.1904(100)$ \\
\hline & 20 & $385.19046(100), 240.13764(99)$ \\
\hline & 40 & $\begin{array}{l}\text { 144.04374(100), 240.13785(97), } \\
55.05416(26), 97.10072(11)\end{array}$ \\
\hline \multirow{3}{*}{$\begin{array}{l}\text { BB-22 7-hydroxyisoquinoline } \\
\text { isomer }\end{array}$} & 10 & $385.1904(100)$ \\
\hline & 20 & $385.19046(100), 240.13764(99)$ \\
\hline & 40 & $\begin{array}{l}\text { 144.04374(100), 240.13785(97), } \\
55.05416(26), 97.10072(11)\end{array}$ \\
\hline \multirow{3}{*}{$\begin{array}{l}\text { BB-22 7-hydroxyquinoline } \\
\text { isomer }\end{array}$} & 10 & $385.19058(100), 240.13757(57)$ \\
\hline & 20 & $240.13766(100)$ \\
\hline & 40 & $\begin{array}{l}\text { 144.04389(100), 240.13771(84), } \\
55.05412(33), 97.10106(11)\end{array}$ \\
\hline \multirow{3}{*}{$\begin{array}{c}\text { BB-22 8-hydroxyisoquinoline } \\
\text { isomer }\end{array}$} & 10 & $385.1909(100), 240.13799(54)$ \\
\hline & 20 & $240.13781(100)$ \\
\hline & 40 & $\begin{array}{l}\text { 144.04402(100), 240.138(84), } \\
55.05416(31), 97.10108(13)\end{array}$ \\
\hline \multirow[b]{2}{*}{$\begin{array}{l}\text { AM2201 2-hydroxyindole } \\
\text { metabolite }\end{array}$} & 10 & $376.17073(100)$ \\
\hline & 20 & $\begin{array}{l}\text { 270.09134(100), 358.16017(98), } \\
\text { 376.17073(94), 155.04914(26), } \\
\text { 282.09134(17), 252.08078(16), } \\
338.15508(12), 172.0393(11)\end{array}$ \\
\hline
\end{tabular}




\begin{tabular}{|c|c|c|}
\hline & 40 & $\begin{array}{l}252.08078(100), 270.09134(59), \\
155.04914(13), 172.0393(12), \\
127.05423(10), 41.03858(10)\end{array}$ \\
\hline \multirow{3}{*}{$\begin{array}{l}\text { AM2201 5-hydroxyindole } \\
\text { metabolite }\end{array}$} & 10 & $376.17011(100)$ \\
\hline & 20 & $\begin{array}{l}376.17073(100), 155.0486(70), \\
248.10677(16)\end{array}$ \\
\hline & 40 & $\begin{array}{l}155.04842(100), 127.05373(95), \\
160.03877(17), 248.10863(14)\end{array}$ \\
\hline \multirow{3}{*}{$\begin{array}{l}\text { AM2201 6-hydroxyindole } \\
\text { metabolite }\end{array}$} & 10 & $376.16995(100)$ \\
\hline & 20 & 155.0484(100), 376.17001(86) \\
\hline & 40 & $155.04842(100), 127.05364(98)$ \\
\hline \multirow{3}{*}{$\begin{array}{l}\text { AM2201 7-hydroxyindole } \\
\text { metabolite }\end{array}$} & 10 & $232.11279(100)$ \\
\hline & 20 & $232.11267(100)$ \\
\hline & 40 & $144.04382(100), 232.11275(71)$ \\
\hline \multirow{3}{*}{$\begin{array}{l}\text { AM2201 N-(4-hydroxypentyl) } \\
\text { metabolite }\end{array}$} & 10 & $376.17047(100), 155.04854(11)$ \\
\hline & 20 & $155.0488(100), 376.16991(68)$ \\
\hline & 40 & $\begin{array}{l}\text { 155.04874(100), 127.05393(86), } \\
144.04407(16)\end{array}$ \\
\hline \multirow{3}{*}{ EAM2201 } & 10 & $388.20657(100)$ \\
\hline & 20 & $\begin{array}{l}388.20703(100), 183.07974(53), \\
232.11256(21)\end{array}$ \\
\hline & 40 & $\begin{array}{l}183.07995(100), 155.08479(48), \\
232.11249(30), 144.04395(29), \\
153.06945(21)\end{array}$ \\
\hline \multirow{3}{*}{ NM2201 } & 10 & $232.11279(100)$ \\
\hline & 20 & $232.11267(100)$ \\
\hline & 40 & $144.04382(100), 232.11275(71)$ \\
\hline \multirow{3}{*}{$\begin{array}{l}\text { AM2201 N-(3-chloropentyl) } \\
\text { isomer }\end{array}$} & 10 & $376.14584(100)$ \\
\hline & 20 & $\begin{array}{l}376.14585(100), 155.04856(73), \\
248.08291(18)\end{array}$ \\
\hline & 40 & $\begin{array}{l}\text { 155.04848(100), 127.05385(94), } \\
144.04371(11), 212.10635(10)\end{array}$ \\
\hline \multirow{3}{*}{$\begin{array}{c}\text { MAM2201 N-(2-fluoropentyl) } \\
\text { isomer }\end{array}$} & 10 & $374.19092(100)$ \\
\hline & 20 & $\begin{array}{l}374.19136(100), 169.06436(74), \\
232.11316(32)\end{array}$ \\
\hline & 40 & $\begin{array}{l}\text { 169.06435(100), 141.06934(93), } \\
232.11267(32), 144.04394(17)\end{array}$ \\
\hline \multirow{3}{*}{$\begin{array}{c}\text { MAM2201 N-(3-fluoropentyl) } \\
\text { isomer }\end{array}$} & 10 & $374.19058(100)$ \\
\hline & 20 & $\begin{array}{l}\text { 374.19113(100), 169.06408(73), } \\
232.11293(25)\end{array}$ \\
\hline & 40 & $\begin{array}{l}\text { 169.06415(100), 141.06949(86), } \\
232.11261(25), 144.04376(15)\end{array}$ \\
\hline
\end{tabular}




\begin{tabular}{|c|c|c|}
\hline \multirow{3}{*}{$\begin{array}{l}\text { MAM2201 N-(4-hydroxypentyl) } \\
\text { metabolite }\end{array}$} & 10 & $390.18588(100)$ \\
\hline & 20 & $\begin{array}{l}\text { 169.06401(100), 390.18626(92), } \\
\text { 248.10786(14) }\end{array}$ \\
\hline & 40 & $\begin{array}{l}\text { 169.06397(100), } 141.06913(71), \\
144.04338(23)\end{array}$ \\
\hline \multirow{3}{*}{$\begin{array}{l}\text { MAM2201 N-(5-chloropentyl) } \\
\text { analog }\end{array}$} & 10 & $390.16182(100)$ \\
\hline & 20 & $\begin{array}{l}\text { 390.16184(100), 169.0643(57), } \\
248.08311(21)\end{array}$ \\
\hline & 40 & $\begin{array}{l}\text { 169.0643(100), 141.06948(77), } \\
248.08329(21), 144.04415(20)\end{array}$ \\
\hline \multirow{3}{*}{$\begin{array}{l}\text { MAM2201 N-(5-chloropentyl) } \\
\text { analog-d5 }\end{array}$} & 10 & $395.19306(100)$ \\
\hline & 20 & $\begin{array}{l}\text { 395.19318(100), 169.06417(42), } \\
253.11452(12), 170.07067(11)\end{array}$ \\
\hline & 40 & $\begin{array}{l}\text { 169.0642(100), 141.0695(69), } \\
170.07058(26), 253.11489(16), \\
142.07587(15)\end{array}$ \\
\hline \multirow{3}{*}{$\begin{array}{l}\text { MAM2201 N-pentanoic acid } \\
\text { metabolite }\end{array}$} & 10 & $386.17497(100), 169.06486(12)$ \\
\hline & 20 & $\begin{array}{l}\text { 169.06409(100), 386.17501(59), } \\
244.09626(10)\end{array}$ \\
\hline & 40 & $\begin{array}{l}\text { 169.06401(100), 141.06942(74), } \\
144.04331(15)\end{array}$ \\
\hline \multirow{3}{*}{$\begin{array}{l}( \pm)-U R-144 \text { N-(4- } \\
\text { hydroxypentyl) metabolite }\end{array}$} & 10 & $328.22697(100), 125.09588(18)$ \\
\hline & 20 & $125.09583(100), 328.22643(24)$ \\
\hline & 40 & $\begin{array}{l}\text { 55.05414(100), 125.09539(66), } \\
57.06983(52), 69.0697(48), \\
97.10102(43)\end{array}$ \\
\hline \multirow{3}{*}{ UR-144 Degradant } & 10 & $312.23111(100), 214.12199(21)$ \\
\hline & 20 & $214.12167(100), 312.23172(19)$ \\
\hline & 40 & $\begin{array}{l}\text { 144.044(100), 214.12215(47), } \\
43.05436(18), 116.04933(11)\end{array}$ \\
\hline \multirow{3}{*}{$\begin{array}{l}\text { UR-144 Degradant N-pentanoic } \\
\text { acid metabolite }\end{array}$} & 10 & $342.20616(100), 244.09637(15)$ \\
\hline & 20 & $244.09646(100), 342.20644(29)$ \\
\hline & 40 & $\begin{array}{l}\text { 144.04413(100), 55.05408(52), } \\
\text { 244.09673(36), 101.05909(20), } \\
83.04897(19)\end{array}$ \\
\hline \multirow{4}{*}{$\begin{array}{l}\text { UR-144 N-(2-chloropentyl) } \\
\text { analog }\end{array}$} & 10 & $346.19287(100)$ \\
\hline & 20 & $\begin{array}{l}\text { 346.19308(100), 125.0959(45), } \\
248.08341(19), 328.18257(11)\end{array}$ \\
\hline & 40 & $\begin{array}{l}\text { 55.05415(100), 144.04412(69), } \\
\text { 125.09582(63), 97.10111(42), } \\
57.06971(40)\end{array}$ \\
\hline & 10 & $328.22705(100)$ \\
\hline
\end{tabular}




\begin{tabular}{|c|c|c|}
\hline \multirow[b]{2}{*}{$\begin{array}{l}\text { UR-144 N-(2-hydroxypentyl) } \\
\text { metabolite }\end{array}$} & 20 & $\begin{array}{l}328.22711(100), 125.09568(62), \\
230.11697(17), 310.21609(12)\end{array}$ \\
\hline & 40 & $\begin{array}{l}\text { 55.05424(100), 144.04439(53), } \\
\text { 125.09598(51), 57.06973(41), } \\
97.10065(39)\end{array}$ \\
\hline \multirow{3}{*}{$\begin{array}{l}\text { UR-144 N-(3-chloropentyl) } \\
\text { analog }\end{array}$} & 10 & $346.19293(100)$ \\
\hline & 20 & $\begin{array}{l}346.19274(100), 125.09581(43), \\
248.08341(16)\end{array}$ \\
\hline & 40 & $\begin{array}{l}\text { 55.05419(100), 125.09577(73), } \\
\text { 69.06952(52), 97.10066(46), } \\
57.07002(43)\end{array}$ \\
\hline \multirow{3}{*}{$\begin{array}{l}\text { UR-144 N-(4-chloropentyl) } \\
\text { analog }\end{array}$} & 10 & $346.19319(100)$ \\
\hline & 20 & $\begin{array}{l}346.19326(100), 125.09576(43), \\
248.08309(14)\end{array}$ \\
\hline & 40 & $\begin{array}{l}55.05427(100), 125.09559(75), \\
69.06957(59), 97.101(46), 57.06979(41)\end{array}$ \\
\hline \multirow{3}{*}{$\begin{array}{l}\text { UR-144 N-(5-bromopentyl) } \\
\text { analog }\end{array}$} & 10 & $390.14256(100)$ \\
\hline & 20 & $\begin{array}{l}390.14259(100), 125.09586(31), \\
292.03295(13)\end{array}$ \\
\hline & 40 & $\begin{array}{l}\text { 55.05432(100), 125.09593(88), } \\
\text { 69.06954(52), 97.10085(50), } \\
\text { 292.03236(44) }\end{array}$ \\
\hline \multirow{3}{*}{$\begin{array}{l}\text { UR-144 N-(5-chloropentyl) } \\
\text { analog }\end{array}$} & 10 & $346.19251(100)$ \\
\hline & 20 & $\begin{array}{l}346.19311(100), 125.09577(42), \\
248.0839(16)\end{array}$ \\
\hline & 40 & $\begin{array}{l}\text { 55.05428(100), 125.09563(65), } \\
\text { 144.04417(44), 97.1009(44), } \\
57.06973(42)\end{array}$ \\
\hline \multirow{3}{*}{$\begin{array}{l}\text { UR-144 N-(5-hydroxypentyl) } \\
\text { metabolite }\end{array}$} & 10 & $328.22691(100), 125.09596(27)$ \\
\hline & 20 & 125.09581(100), 328.22682(17) \\
\hline & 40 & $\begin{array}{l}\text { 55.05412(100), 125.09583(69), } \\
57.06974(48), 97.10075(41), \\
69.06929(39)\end{array}$ \\
\hline \multirow{3}{*}{$\begin{array}{c}\text { UR-144 N-(5-hydroxypentyl) } \beta \text { - } \\
\text { D-Glucuronide }\end{array}$} & 10 & $\begin{array}{l}\text { 504.25802(100), 328.22683(31), } \\
125.09491(12)\end{array}$ \\
\hline & 20 & \\
\hline & 40 & \\
\hline \multirow{3}{*}{$\begin{array}{l}\text { UR-144 N-pentanoic acid } \\
\text { metabolite }\end{array}$} & 10 & $342.2062(100), 125.09622(11)$ \\
\hline & 20 & $\begin{array}{l}125.09588(100), 342.20637(55), \\
244.09565(13)\end{array}$ \\
\hline & 40 & $\begin{array}{l}\text { 55.05408(100), 125.09563(66), } \\
97.10091(39), 57.06983(33), \\
144.04386(32)\end{array}$ \\
\hline
\end{tabular}




\begin{tabular}{|c|c|c|}
\hline \multirow{3}{*}{$\begin{array}{l}\text { UR-144 N-(5-methylhexyl) } \\
\text { analog }\end{array}$} & 10 & $340.26263(100)$ \\
\hline & 20 & $\begin{array}{l}340.26329(100), 125.09567(36) \\
242.15289(13)\end{array}$ \\
\hline & 40 & $\begin{array}{l}55.0542(100), 125.09579(79), \\
57.06972(61), 144.04445(50), \\
97.10105(48)\end{array}$ \\
\hline \multirow{3}{*}{ UR-144 N-heptyl analog } & 10 & $340.26232(100)$ \\
\hline & 20 & $\begin{array}{l}340.26306(100), 125.0958(42), \\
242.15301(15)\end{array}$ \\
\hline & 40 & $\begin{array}{l}55.05411(100), 125.0956(73), \\
57.06961(61), 144.04446(48), \\
97.10088(43)\end{array}$ \\
\hline \multirow{3}{*}{ FUB-144 } & 10 & $350.19059(100)$ \\
\hline & 20 & $\begin{array}{l}350.19098(100), 125.09571(51), \\
109.04444(17), 252.08119(16)\end{array}$ \\
\hline & 40 & $\begin{array}{l}\text { 109.04452(100), 55.05414(20), } \\
125.0953(13)\end{array}$ \\
\hline \multirow{3}{*}{$\begin{array}{l}\text { XLR11 6-hydroxyindole } \\
\text { metabolite }\end{array}$} & 10 & $346.21725(100)$ \\
\hline & 20 & $\begin{array}{l}346.21741(100), 125.09575(85), \\
248.10762(11)\end{array}$ \\
\hline & 40 & $\begin{array}{l}55.05391(100), 125.09544(68), \\
97.10079(44), 57.06956(39), \\
69.06901(28)\end{array}$ \\
\hline \multirow{3}{*}{ XLR11 Degradant } & 10 & $330.22234(100)$ \\
\hline & 20 & $\begin{array}{l}330.22269(100), 125.09581(55) \\
232.11312(26), 312.21194(13)\end{array}$ \\
\hline & 40 & $\begin{array}{l}55.05432(100), 232.1134(55), \\
125.09591(50), 57.06977(43), \\
97.10095(35)\end{array}$ \\
\hline \multirow{3}{*}{$\begin{array}{l}\text { XLR11 N-(2-fluoropentyl) } \\
\text { isomer }\end{array}$} & 10 & $330.22234(100)$ \\
\hline & 20 & $\begin{array}{l}330.22269(100), 125.09581(55), \\
232.11312(26), 312.21194(13)\end{array}$ \\
\hline & 40 & $\begin{array}{l}55.05432(100), 232.1134(55), \\
125.09591(50), 57.06977(43), \\
97.10095(35)\end{array}$ \\
\hline \multirow{4}{*}{$\begin{array}{l}\text { XLR11 N-(3-fluoropentyl) } \\
\text { isomer }\end{array}$} & 10 & $330.2216(100)$ \\
\hline & 20 & $\begin{array}{l}330.2227(100), 125.09588(52), \\
232.11311(22), 312.21146(11)\end{array}$ \\
\hline & 40 & $\begin{array}{l}55.05416(100), 125.09562(54), \\
232.11309(45), 57.06985(44), \\
97.1008(37)\end{array}$ \\
\hline & 10 & $330.22193(100)$ \\
\hline
\end{tabular}




\begin{tabular}{|c|c|c|}
\hline \multirow{2}{*}{$\begin{array}{l}\text { XLR11 N-(4-fluoropentyl) } \\
\text { isomer }\end{array}$} & 20 & $\begin{array}{l}330.22271(100), 125.09573(55), \\
232.11296(19)\end{array}$ \\
\hline & 40 & $\begin{array}{l}\text { 55.05408(100), 125.09551(59), } \\
\text { 144.04421(47), 57.06976(45), } \\
97.10081(39)\end{array}$ \\
\hline \multirow{3}{*}{$\begin{array}{l}\text { XLR11 N-(4-hydroxypentyl) } \\
\text { metabolite }\end{array}$} & 10 & $346.21768(100), 248.10834(12)$ \\
\hline & 20 & $248.10778(100), 346.21761(41)$ \\
\hline & 40 & $\begin{array}{l}\text { 144.04379(100), 248.10792(30), } \\
67.05413(19), 87.06026(12)\end{array}$ \\
\hline \multirow{3}{*}{ XLR11 N-(4-pentenyl) analog } & 10 & $310.21594(100)$ \\
\hline & 20 & $\begin{array}{l}310.21638(100), 125.09583(98), \\
212.10656(35), 292.20574(14), \\
97.10107(12)\end{array}$ \\
\hline & 40 & $\begin{array}{l}\text { 55.05428(100), 57.07(45), } \\
\text { 125.09567(34),144.04478(34), } \\
97.10083(31)\end{array}$ \\
\hline \multirow{3}{*}{ XLR12 } & 10 & $352.18872(100)$ \\
\hline & 20 & $\begin{array}{l}352.18845(100), 125.09588(34), \\
254.07835(19), 334.17718(12)\end{array}$ \\
\hline & 40 & $\begin{array}{l}254.07881(100), 55.05415(90), \\
125.09597(61), 144.04426(45), \\
97.10076(40)\end{array}$ \\
\hline \multirow{3}{*}{ Acetyl fentanyl } & 10 & $323.21099(100)$ \\
\hline & 20 & $\begin{array}{l}\text { 188.14277(100), 323.21137(84), } \\
105.06997(11)\end{array}$ \\
\hline & 40 & $105.06983(100), 188.14296(18)$ \\
\hline \multirow{3}{*}{ Acetyl norfentanyl } & 10 & $219.1482(100), 84.08043(44)$ \\
\hline & 20 & $\begin{array}{l}\text { 84.08044(100), 56.04947(20), } \\
55.05437(11)\end{array}$ \\
\hline & 40 & $\begin{array}{l}55.05443(100), 56.04949(55), \\
84.08061(25), 42.03397(15), \\
94.06508(14)\end{array}$ \\
\hline \multirow{3}{*}{ Butyryl fentanyl } & 10 & $351.24227(100)$ \\
\hline & 20 & $351.24284(100), 188.14289(95)$ \\
\hline & 40 & $105.06976(100), 188.14324(33)$ \\
\hline \multirow{3}{*}{ para-Fluorofentanyl } & 10 & $355.21752(100)$ \\
\hline & 20 & $355.21766(100), 188.14321(78)$ \\
\hline & 40 & 105.06974(100), 188.14301(32) \\
\hline \multirow[b]{2}{*}{ AH 7921} & 10 & $329.118(100), 284.06034(23)$ \\
\hline & 20 & $\begin{array}{l}284.06043(100), 172.95501(34), \\
46.06524(26), 95.08563(18), \\
189.98181(18)\end{array}$ \\
\hline
\end{tabular}




\begin{tabular}{|c|c|c|}
\hline & 40 & $\begin{array}{l}\text { 172.95528(100), 95.0855(47), } \\
46.06533(24), 144.96023(21), \\
67.05401(18)\end{array}$ \\
\hline \multirow{3}{*}{ ATM4 4-acetoxy analog } & 10 & $\begin{array}{l}\text { 396.18034(100), 378.16981(33), } \\
354.17022(27), 305.11737(12)\end{array}$ \\
\hline & 20 & $\begin{array}{l}281.11728(100), 354.16972(76), \\
305.11693(58), 249.09069(32), \\
323.12785(19)\end{array}$ \\
\hline & 40 & $\begin{array}{l}221.09551(100), 249.09063(40), \\
217.06478(19), 234.06732(17), \\
266.09372(15)\end{array}$ \\
\hline \multirow{3}{*}{ 3-hydroxy Phenazepam } & 10 & $364.96869(100)$ \\
\hline & 20 & \\
\hline & 40 & \\
\hline \multirow{3}{*}{ Bromazepam } & 10 & $316.00793(100)$ \\
\hline & 20 & $\begin{array}{l}316.008(100), 288.01261(27), \\
209.09459(18), 261.00239(13), \\
182.083(11)\end{array}$ \\
\hline & 40 & $\begin{array}{l}\text { 182.08334(100), 208.08578(40), } \\
209.09353(38), 259.99373(30), \\
183.97511(19)\end{array}$ \\
\hline \multirow[b]{3}{*}{ Delorazepam } & 10 & $305.02429(100), 304.28364(10)$ \\
\hline & 20 & $305.02423(100)$ \\
\hline & 40 & $\begin{array}{l}140.02671(100), 206.08231(41), \\
165.02068(29), 241.05169(26), \\
228.0446(26)\end{array}$ \\
\hline \multirow[b]{3}{*}{ Diclazepam } & 10 & $319.03997(100)$ \\
\hline & 20 & $319.03975(100)$ \\
\hline & 40 & $\begin{array}{l}227.04904(100), 154.04173(68), \\
125.01521(28), 275.01344(28), \\
220.09816(26)\end{array}$ \\
\hline \multirow[b]{3}{*}{ Etizolam } & 10 & $343.07775(100)$ \\
\hline & 20 & $343.07742(100), 314.03804(26)$ \\
\hline & 40 & $\begin{array}{l}314.03875(100), 259.02108(47), \\
138.03513(25), 295.07326(23), \\
224.05003(23)\end{array}$ \\
\hline \multirow[b]{3}{*}{ Flubromazepam } & 10 & $333.00333(100)$ \\
\hline & 20 & $333.00333(100)$ \\
\hline & 40 & $\begin{array}{l}\text { 226.09008(100), 183.97564(86), } \\
206.08385(47), 105.0573(35), \\
179.07295(26), 208.97089(23), \\
109.0448(17), 257.99129(17), \\
333.00333(16), 104.04948(15),\end{array}$ \\
\hline
\end{tabular}




\begin{tabular}{|c|c|c|}
\hline & & $\begin{array}{l}225.08225(15), 198.07135(15), \\
205.07602(13), 130.05255(12) \\
211.07918(10)\end{array}$ \\
\hline \multirow{3}{*}{ Pyrazolam } & 10 & $354.03416(100), 230.13916(38)$ \\
\hline & 20 & $354.03429(100), 230.13921(41)$ \\
\hline & 40 & \\
\hline \multirow{3}{*}{ 1'-naphthoyl-2-methylindole } & 10 & $286.12244(100), 155.04888(18)$ \\
\hline & 20 & $\begin{array}{l}\text { 155.0491(100), 158.05984(36), } \\
286.12264(18), 127.05433(10)\end{array}$ \\
\hline & 40 & $\begin{array}{l}127.05423(100), 158.05972(19), \\
155.04895(17)\end{array}$ \\
\hline \multirow{3}{*}{ 5-IT } & 10 & $158.09626(100), 130.065(22)$ \\
\hline & 20 & $\begin{array}{l}\text { 130.0651(100), 117.05753(47), } \\
158.09621(36), 143.07208(12)\end{array}$ \\
\hline & 40 & $\begin{array}{l}\text { 130.06497(100), 117.05727(81), } \\
77.03861(48), 103.05426(40), \\
115.05401(31)\end{array}$ \\
\hline \multirow{3}{*}{ 6-IT } & 10 & $158.09626(100), 130.065(22)$ \\
\hline & 20 & $\begin{array}{l}\text { 130.0651(100), 117.05753(47), } \\
\text { 158.09621(36), 143.07208(12) }\end{array}$ \\
\hline & 40 & $\begin{array}{l}\text { 130.06497(100), 117.05727(81), } \\
\text { 77.03861(48), 103.05426(40), } \\
115.05401(31)\end{array}$ \\
\hline \multirow[b]{3}{*}{ A- 834735} & 10 & $340.22671(100)$ \\
\hline & 20 & $125.09588(100), 340.22724(89)$ \\
\hline & 40 & $\begin{array}{l}\text { 55.05402(100), 125.09563(94), } \\
97.10079(50), 69.06952(47), \\
57.06966(41)\end{array}$ \\
\hline \multirow[b]{3}{*}{ A-834735 degredant } & 10 & $340.22761(100), 242.11781(11)$ \\
\hline & 20 & $242.1176(100), 340.22727(54)$ \\
\hline & 40 & $\begin{array}{l}99.08037(100), 242.1172(72), \\
69.06995(64), 144.04399(36), \\
81.0696(36)\end{array}$ \\
\hline \multirow{4}{*}{$\begin{array}{l}\text { methyl-1-(5-fluoropentyl)-1H- } \\
\text { indole-3-Carboxylate }\end{array}$} & 10 & $264.13943(100), 232.11383(27)$ \\
\hline & 20 & $\begin{array}{l}232.1138(100), 144.04486(21), \\
132.08066(20), 264.13996(12)\end{array}$ \\
\hline & 40 & $\begin{array}{l}\text { 144.0446(100), 130.06485(99), } \\
117.05733(68), 116.04948(55), \\
129.05739(31)\end{array}$ \\
\hline & 10 & $272.16343(100)$ \\
\hline
\end{tabular}




\begin{tabular}{|c|c|c|}
\hline \multirow{2}{*}{$\begin{array}{l}\text { methyl-1-(cyclohexylmethyl)- } \\
\text { 1H-indole-3-carboxylate }\end{array}$} & 20 & $\begin{array}{l}240.13756(100), 144.04427(71), \\
272.16492(64), 190.08472(57), \\
176.06989(39)\end{array}$ \\
\hline & 40 & $\begin{array}{l}55.05439(100), 144.04452(49), \\
117.05779(26), 116.04914(25), \\
130.06373(22)\end{array}$ \\
\hline \multirow{3}{*}{$\begin{array}{l}\text { methyl-1-pentyl-1H-indole-3- } \\
\text { Carboxylate }\end{array}$} & 10 & $246.14886(100), 214.12264(38)$ \\
\hline & 20 & $\begin{array}{l}\text { 214.12264(100), 144.04439(36), } \\
132.08078(22), 43.05423(11)\end{array}$ \\
\hline & 40 & \\
\hline \multirow{3}{*}{ ADBICA } & 10 & $327.2067(100), 214.12264(69)$ \\
\hline & 20 & $214.12264(100)$ \\
\hline & 40 & $\begin{array}{l}\text { 214.12264(100), 144.04439(85), } \\
43.05423(13)\end{array}$ \\
\hline \multirow{3}{*}{$\begin{array}{l}\text { ADBICA N-(4-hydroxypentyl) } \\
\text { metabolite }\end{array}$} & 10 & $\begin{array}{l}343.20122(100), 230.11742(67), \\
360.32389(53)\end{array}$ \\
\hline & 20 & $230.11747(100)$ \\
\hline & 40 & $\begin{array}{l}\text { 144.04396(100), 69.06932(39), } \\
230.11599(35)\end{array}$ \\
\hline \multirow{3}{*}{$\begin{array}{l}\text { ADBICA N-(5-hydroxypentyl) } \\
\text { metabolite }\end{array}$} & 10 & $\begin{array}{l}343.20168(100), 230.11757(56), \\
360.32318(42)\end{array}$ \\
\hline & 20 & $230.11703(100)$ \\
\hline & 40 & $\begin{array}{l}144.04409(100), 230.11737(93), \\
69.0699(21)\end{array}$ \\
\hline \multirow{3}{*}{$\begin{array}{l}\text { ADBICA N-pentanoic acid } \\
\text { metabolite }\end{array}$} & 10 & $357.18088(100), 244.09682(53)$ \\
\hline & 20 & $244.09682(100)$ \\
\hline & 40 & $\begin{array}{l}\text { 144.04439(100), 244.09682(95), } \\
55.05423(33), 101.05971(23), \\
83.04914(16)\end{array}$ \\
\hline \multirow{3}{*}{$\begin{array}{l}\text { AM694 N-(5-hydroxypentyl) } \\
\text { metabolite }\end{array}$} & 10 & $434.0607(100), 230.92931(54)$ \\
\hline & 20 & $230.92991(100), 434.06091(12)$ \\
\hline & 40 & $230.93002(100), 202.93537(47)$ \\
\hline \multirow{3}{*}{$\begin{array}{l}\text { AM694 N-pentanoic acid } \\
\text { metabolite }\end{array}$} & 10 & $448.03976(100), 230.9297(35)$ \\
\hline & 20 & $230.92933(100), 448.03941(25)$ \\
\hline & 40 & $230.92981(100), 202.93487(39)$ \\
\hline \multirow{3}{*}{ tetrahydro-Harmine } & 10 & $\begin{array}{l}\text { 188.10729(100), 200.10736(72), } \\
217.13204(10)\end{array}$ \\
\hline & 20 & $\begin{array}{l}188.10712(100), 200.10748(38), \\
173.08421(23), 185.08318(12)\end{array}$ \\
\hline & 40 & $\begin{array}{l}158.06068(100), 145.08847(95), \\
130.06534(94), 173.0838(84), \\
156.081(54)\end{array}$ \\
\hline
\end{tabular}




\begin{tabular}{|c|c|c|}
\hline \multirow{3}{*}{ 5-chloro AB-PINACA } & 10 & $\begin{array}{l}320.152(100), 348.1475(59), \\
249.07871(10)\end{array}$ \\
\hline & 20 & $249.07813(100), 320.15247(90)$ \\
\hline & 40 & $\begin{array}{l}\text { 213.10183(100), 145.0396(67), } \\
\text { 249.07836(46), 69.06954(16), } \\
\text { 193.01531(10) }\end{array}$ \\
\hline \multirow{3}{*}{ 5-fluoro ABICA } & 10 & $232.11377(100), 331.18298(91)$ \\
\hline & 20 & $232.11365(100)$ \\
\hline & 40 & 144.044(100), 232.11323(99) \\
\hline \multirow[b]{3}{*}{ 5-fluoro AB-PINACA } & 10 & $304.18197(100), 332.17688(66)$ \\
\hline & 20 & $233.10847(100), 304.18197(74)$ \\
\hline & 40 & $\begin{array}{l}\text { 145.03964(100), 213.10224(77), } \\
233.10847(56), 177.04587(31), \\
69.06988(30), 41.03858(10)\end{array}$ \\
\hline \multirow{3}{*}{$\begin{array}{l}\text { 5-fluoro AB-PINACA N-(4- } \\
\text { hydroxypentyl) metabolite }\end{array}$} & 10 & $320.1788(100), 348.17199(71)$ \\
\hline & 20 & $249.10329(100), 320.17775(69)$ \\
\hline & 40 & \\
\hline \multirow{3}{*}{ 5-fluoro ADBICA } & 10 & $345.19866(100), 232.11447(55)$ \\
\hline & 20 & 232.11392(100) \\
\hline & 40 & $232.11437(100), 144.0446(56)$ \\
\hline \multirow[b]{3}{*}{ 5-fluoro ADB-PINACA } & 10 & $318.19782(100), 346.19342(82)$ \\
\hline & 20 & $233.10885(100), 318.19848(94)$ \\
\hline & 40 & $\begin{array}{l}213.10263(100), 233.10929(99), \\
145.03971(85), 69.06982(35), \\
177.04638(34)\end{array}$ \\
\hline \multirow{3}{*}{ 5-fluoro AMB } & 10 & $\begin{array}{l}364.20455(100), 304.18299(73), \\
233.10906(27), 332.17759(15)\end{array}$ \\
\hline & 20 & $\begin{array}{l}233.10919(100), 304.18314(41), \\
213.10265(12)\end{array}$ \\
\hline & 40 & $\begin{array}{l}\text { 145.03985(100), 213.10293(49), } \\
233.10884(26), 69.06996(24), \\
177.04607(23)\end{array}$ \\
\hline \multirow[b]{3}{*}{ 5-fluoro MN-18 } & 10 & $233.10904(100), 376.18315(86)$ \\
\hline & 20 & $233.1089(100), 213.10218(19)$ \\
\hline & 40 & $\begin{array}{l}\text { 145.03998(100), 213.10267(29), } \\
\text { 69.06989(19), 177.04602(19), } \\
41.03896(11)\end{array}$ \\
\hline \multirow{3}{*}{$\begin{array}{l}\text { 5-fluoro-AKB48 N-(4- } \\
\text { hydroxypentyl) metabolite }\end{array}$} & 10 & $400.24064(100), 135.11715(46)$ \\
\hline & 20 & $135.11713(100)$ \\
\hline & 40 & $135.11701(100)$ \\
\hline 5-fluoro-THJ & 10 & $377.1785(100)$ \\
\hline
\end{tabular}




\begin{tabular}{|c|c|c|}
\hline & 20 & $\begin{array}{l}233.10886(100), 377.17824(39), \\
359.16801(34), 213.10224(16)\end{array}$ \\
\hline & 40 & $\begin{array}{l}\text { 145.03996(100), 213.10207(41), } \\
\text { 69.06995(20), 177.04614(20), } \\
359.16849(19)\end{array}$ \\
\hline \multirow{3}{*}{ AB-005 } & 10 & $353.25935(100)$ \\
\hline & 20 & $\begin{array}{l}\text { 112.11227(100), 353.25947(84), } \\
125.09627(56), 98.09656(54), \\
256.16996(36)\end{array}$ \\
\hline & 40 & $\begin{array}{l}\text { 98.09672(100), 112.11252(49), } \\
\text { 125.09617(15), 58.0654(14), } \\
\text { 55.05447(11) }\end{array}$ \\
\hline \multirow{3}{*}{ AB-005 azepane isomer } & 10 & $353.25874(100), 112.11208(27)$ \\
\hline & 20 & $112.11208(100)$ \\
\hline & 40 & $112.11208(100), 58.06513(27)$ \\
\hline \multirow{3}{*}{ AB-CHMINACA } & 10 & $\begin{array}{l}340.20319(100), 312.20749(92), \\
241.13421(11)\end{array}$ \\
\hline & 20 & 241.13399(100), 312.20783(83) \\
\hline & 40 & $\begin{array}{l}\text { 241.13399(100), 145.03959(97), } \\
55.05414(12)\end{array}$ \\
\hline \multirow{3}{*}{ AB-FUBINACA } & 10 & $\begin{array}{l}324.15153(100), 352.14816(50), \\
253.07882(12)\end{array}$ \\
\hline & 20 & $253.07806(100), 324.1513(69)$ \\
\hline & 40 & $109.04533(100), 253.07734(26)$ \\
\hline \multirow{3}{*}{$\begin{array}{l}\text { AB-FUBINACA 2-fluorobenzyl } \\
\text { isomer }\end{array}$} & 10 & $\begin{array}{l}324.15178(100), 352.14623(69), \\
253.07786(14)\end{array}$ \\
\hline & 20 & $253.0776(100), 324.15151(47)$ \\
\hline & 40 & $109.04479(100), 253.07769(25)$ \\
\hline \multirow{3}{*}{$\begin{array}{l}\text { AB-FUBINACA 3-fluorobenzyl } \\
\text { isomer }\end{array}$} & 10 & $\begin{array}{l}324.15178(100), 352.14623(69), \\
253.07786(14)\end{array}$ \\
\hline & 20 & $253.0776(100), 324.15151(47)$ \\
\hline & 40 & $109.04479(100), 253.07769(25)$ \\
\hline \multirow{3}{*}{ AB-FUBINACA isomer 1} & 10 & $\begin{array}{l}324.15185(100), 352.14689(48), \\
253.07617(18)\end{array}$ \\
\hline & 20 & $253.07734(100), 324.15149(33)$ \\
\hline & 40 & 109.04494(100), 253.07802(18) \\
\hline \multirow{3}{*}{ AB-FUBINACA isomer 2} & 10 & $\begin{array}{l}324.15178(100), 352.14623(69), \\
253.07786(14)\end{array}$ \\
\hline & 20 & $253.0776(100), 324.15151(47)$ \\
\hline & 40 & $109.04479(100), 253.07769(25)$ \\
\hline \multirow[t]{2}{*}{ AB-FUBINACA isomer 5} & 10 & $\begin{array}{l}352.14558(100), 324.15067(29), \\
253.07717(15)\end{array}$ \\
\hline & 20 & $253.07717(100), 324.15067(26)$ \\
\hline
\end{tabular}




\begin{tabular}{|c|c|c|}
\hline & 40 & $109.0448(100), 253.07717(21)$ \\
\hline \multirow{3}{*}{ AB-PINACA } & 10 & $\begin{array}{l}286.19185(100), 314.187(46), \\
215.11826(16)\end{array}$ \\
\hline & 20 & $215.11781(100), 286.19223(37)$ \\
\hline & 40 & $145.03994(100), 215.1182(76)$ \\
\hline \multirow{3}{*}{$\begin{array}{l}\text { AB-PINACA N-(2- } \\
\text { fluoropentyl) isomer }\end{array}$} & 10 & $\begin{array}{l}304.18241(100), 332.17836(49), \\
233.10847(10)\end{array}$ \\
\hline & 20 & $233.10894(100), 304.18257(65)$ \\
\hline & 40 & $233.10887(100), 145.03961(39)$ \\
\hline \multirow{3}{*}{$\begin{array}{l}\text { AB-PINACA N-(4- } \\
\text { fluoropentyl) isomer }\end{array}$} & 10 & $304.18228(100), 332.17763(56)$ \\
\hline & 20 & $233.1088(100), 304.1823(57)$ \\
\hline & 40 & $\begin{array}{l}\text { 145.03964(100), 213.10229(90), } \\
233.10886(80), 69.06993(47), \\
177.0455(34)\end{array}$ \\
\hline \multirow{3}{*}{$\begin{array}{l}\text { AB-PINACA N-(4- } \\
\text { hydroxypentyl) metabolite }\end{array}$} & 10 & $302.18699(100), 330.18267(86)$ \\
\hline & 20 & $\begin{array}{l}302.18674(100), 231.11332(72), \\
213.10217(57)\end{array}$ \\
\hline & 40 & $\begin{array}{l}\text { 213.1022(100), 145.04073(65), } \\
69.06983(25), 175.04903(18)\end{array}$ \\
\hline \multirow{3}{*}{$\begin{array}{l}\text { AB-PINACA N-(5- } \\
\text { hydroxypentyl) metabolite }\end{array}$} & 10 & $302.18699(100), 330.18267(86)$ \\
\hline & 20 & $\begin{array}{l}302.18674(100), 231.11332(72), \\
213.10217(57)\end{array}$ \\
\hline & 40 & $\begin{array}{l}\text { 213.1022(100), 145.04073(65), } \\
69.06983(25), 175.04903(18)\end{array}$ \\
\hline \multirow{3}{*}{$\begin{array}{l}\text { AB-PINACA pentanoic acid } \\
\text { metabolite }\end{array}$} & 10 & $\begin{array}{l}316.16624(100), 344.16098(71), \\
360.32446(66)\end{array}$ \\
\hline & 20 & $\begin{array}{l}316.16593(100), 245.09243(81), \\
298.15558(69), 217.0968(38), \\
227.07955(21)\end{array}$ \\
\hline & 40 & $\begin{array}{l}217.09748(100), 227.08192(88), \\
199.08861(19), 145.03973(18), \\
175.05113(12)\end{array}$ \\
\hline \multirow{3}{*}{$\begin{array}{l}\text { AKB48 N-(5-fluoropentyl) } \\
\text { analog }\end{array}$} & 10 & $384.24534(100), 135.11694(53)$ \\
\hline & 20 & $135.11696(100)$ \\
\hline & 40 & $135.11701(100), 93.06999(12)$ \\
\hline \multirow{3}{*}{$\mathrm{MN}-18$} & 10 & $215.11827(100), 358.19236(52)$ \\
\hline & 20 & $215.11816(100)$ \\
\hline & 40 & 145.03972(100), 215.11867(23) \\
\hline \multirow[b]{2}{*}{ THJ } & 10 & $359.18772(100), 215.1181(15)$ \\
\hline & 20 & $\begin{array}{l}215.11811(100), 341.17679(23), \\
359.18734(18)\end{array}$ \\
\hline
\end{tabular}




\begin{tabular}{|c|c|c|}
\hline & 40 & $\begin{array}{l}\text { 145.03971(100), 215.1183(33), } \\
341.17646(11)\end{array}$ \\
\hline \multirow{3}{*}{ THJ 018} & 10 & $215.11829(100), 343.18154(87)$ \\
\hline & 20 & $215.11774(100)$ \\
\hline & 40 & $145.03956(100), 215.11782(11)$ \\
\hline \multirow[b]{3}{*}{ THJ 2201} & 10 & $361.17225(100), 233.10866(78)$ \\
\hline & 20 & $233.10873(100), 213.10228(29)$ \\
\hline & 40 & $\begin{array}{l}\text { 145.03963(100), 213.10312(17), } \\
\text { 69.07009(14), 177.04602(13), } \\
41.03897(12)\end{array}$ \\
\hline \multirow{3}{*}{ ADB-FUBINACA } & 10 & $\begin{array}{l}338.16723(100), 366.1627(65), \\
253.07688(11)\end{array}$ \\
\hline & 20 & $253.07754(100), 338.16796(90)$ \\
\hline & 40 & 109.04492(100), 253.07694(35) \\
\hline \multirow{3}{*}{ ADB-PINACA } & 10 & $\begin{array}{l}300.20752(100), 328.20255(67), \\
215.11832(11)\end{array}$ \\
\hline & 20 & $215.11821(100), 300.20782(56)$ \\
\hline & 40 & $215.11819(100), 145.03969(92)$ \\
\hline \multirow{3}{*}{ ADB-PINACA isomer 1} & 10 & $\begin{array}{l}300.20778(100), 328.20252(82), \\
215.11795(11)\end{array}$ \\
\hline & 20 & $215.11785(100), 300.20765(58)$ \\
\hline & 40 & $215.11816(100), 145.03973(89)$ \\
\hline \multirow{3}{*}{ ADB-PINACA isomer 2} & 10 & $\begin{array}{l}300.20751(100), 328.20283(45), \\
215.11827(10)\end{array}$ \\
\hline & 20 & $215.11813(100), 300.20756(45)$ \\
\hline & 40 & $145.03922(100), 215.11823(90)$ \\
\hline \multirow{3}{*}{ ADB-PINACA isomer 3} & 10 & $\begin{array}{l}300.20769(100), 328.20257(48), \\
215.11754(17)\end{array}$ \\
\hline & 20 & $215.11823(100), 300.20699(23)$ \\
\hline & 40 & $145.03974(100), 215.11786(82)$ \\
\hline \multirow{3}{*}{$\begin{array}{l}\text { ADB-PINACA N-(4- } \\
\text { hydroxypentyl) metabolite }\end{array}$} & 10 & $344.19823(100), 316.20279(99)$ \\
\hline & 20 & $\begin{array}{l}316.20225(100), 231.11382(90), \\
213.10295(33)\end{array}$ \\
\hline & 40 & $\begin{array}{l}213.10198(100), 145.03946(64), \\
69.06984(38), 175.0488(12)\end{array}$ \\
\hline \multirow{4}{*}{$\begin{array}{l}\text { ADB-PINACA N-(5- } \\
\text { hydroxypentyl) metabolite }\end{array}$} & 10 & $344.19704(100), 316.2023(83)$ \\
\hline & 20 & $\begin{array}{l}316.20226(100), 231.11287(73), \\
213.10263(41)\end{array}$ \\
\hline & 40 & $\begin{array}{l}\text { 213.10193(100), 145.03951(37), } \\
69.07013(16), 175.05033(13)\end{array}$ \\
\hline & 10 & $330.1825(100), 358.17585(87)$ \\
\hline
\end{tabular}




\begin{tabular}{|c|c|c|}
\hline \multirow{2}{*}{$\begin{array}{l}\text { ADB-PINACA pentanoic acid } \\
\text { metabolite }\end{array}$} & 20 & $\begin{array}{l}330.18171(100), 245.09227(54), \\
217.09701(18)\end{array}$ \\
\hline & 40 & $\begin{array}{l}217.09694(100), 227.08119(77), \\
145.03884(14), 199.08577(13)\end{array}$ \\
\hline \multirow{3}{*}{$\begin{array}{l}\text { AKB48 N-(4-fluorobenzyl) } \\
\text { analog }\end{array}$} & 10 & $404.21327(100), 135.11683(90)$ \\
\hline & 20 & $135.11663(100)$ \\
\hline & 40 & $135.11683(100), 93.06988(11)$ \\
\hline \multirow{3}{*}{$\begin{array}{l}\text { AKB48 N-(4-hydroxypentyl) } \\
\text { metabolite }\end{array}$} & 10 & $382.25027(100), 135.11709(39)$ \\
\hline & 20 & $135.11698(100)$ \\
\hline & 40 & $135.11713(100), 93.07001(12)$ \\
\hline \multirow{3}{*}{$\begin{array}{l}\text { AKB48 N-(5-hydroxypentyl) } \\
\text { metabolite }\end{array}$} & 10 & $382.25008(100), 135.11694(33)$ \\
\hline & 20 & $135.1169(100), 382.25017(12)$ \\
\hline & 40 & $135.117(100)$ \\
\hline \multirow{3}{*}{$\begin{array}{c}\text { AKB48 N-pentanoic acid } \\
\text { metabolite }\end{array}$} & 10 & $396.22964(100), 135.11735(39)$ \\
\hline & 20 & $135.11701(100), 396.22989(10)$ \\
\hline & 40 & $135.11694(100), 93.06995(11)$ \\
\hline \multirow{3}{*}{ AMB } & 10 & $\begin{array}{l}346.21373(100), 286.19223(87), \\
215.11817(48), 314.18695(19)\end{array}$ \\
\hline & 20 & $215.11811(100), 286.1922(19)$ \\
\hline & 40 & $145.0399(100), 215.11807(44)$ \\
\hline \multirow{3}{*}{ 4-acetoxy DiPT (hydrochloride) } & 10 & $\begin{array}{l}303.2067(100), 114.12773(24), \\
202.08626(10)\end{array}$ \\
\hline & 20 & $\begin{array}{l}\text { 114.12773(100), 160.07569(63), } \\
202.08626(52), 102.12773(10)\end{array}$ \\
\hline & 40 & $\begin{array}{l}\text { 160.07569(100), 132.08078(17), } \\
72.08078(16), 115.05423(13), \\
114.12773(12)\end{array}$ \\
\hline \multirow{3}{*}{ 4-acetoxy DMT (hydrochloride) } & 10 & $\begin{array}{l}\text { 247.14467(100), 58.0653(97), } \\
202.08648(32), 160.07558(10)\end{array}$ \\
\hline & 20 & $\begin{array}{l}58.0653(100), 160.07575(53), \\
202.08604(12)\end{array}$ \\
\hline & 40 & $\begin{array}{l}58.0653(100), 160.07562(35), \\
115.05413(27), 132.08089(14), \\
117.05807(11)\end{array}$ \\
\hline \multirow{3}{*}{ 4-hydroxy DET } & 10 & $\begin{array}{l}\text { 86.09633(100), 233.16548(40), } \\
160.07596(25)\end{array}$ \\
\hline & 20 & $86.09633(100), 160.07553(63)$ \\
\hline & 40 & $\begin{array}{l}\text { 115.05453(100), 86.09669(80), } \\
160.0759(30), 58.0653(29), \\
117.05774(29)\end{array}$ \\
\hline 4-hydroxy DiPT (hydrochloride) & 10 & $\begin{array}{l}\text { 261.19643(100), 114.12793(74), } \\
160.07606(34)\end{array}$ \\
\hline
\end{tabular}




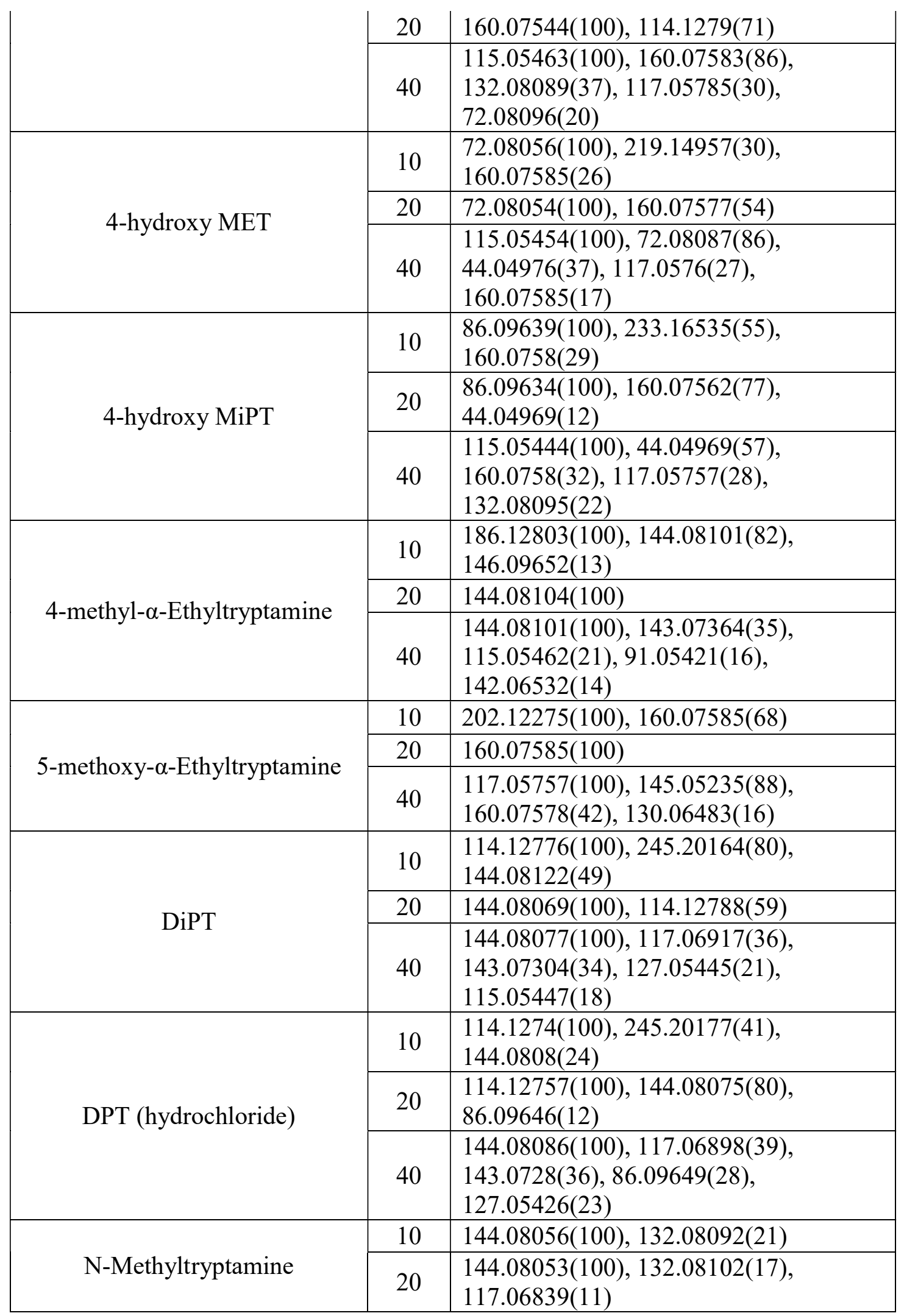




\begin{tabular}{|c|c|c|}
\hline & 40 & $\begin{array}{l}\text { 115.05437(100), 117.06115(79), } \\
\text { 143.07301(69), 91.05434(65), } \\
77.03856(32)\end{array}$ \\
\hline \multirow{3}{*}{ AMT (hydrochloride) } & 10 & $131.06375(100)$ \\
\hline & 20 & $\begin{array}{l}55.0544(100), 131.06379(89), \\
59.99032(26), 89.01698(21), \\
77.01687(18)\end{array}$ \\
\hline & 40 & $\begin{array}{l}59.99027(100), 55.05438(25), \\
71.99048(22)\end{array}$ \\
\hline \multirow{3}{*}{$\begin{array}{l}\text { Methylphenidate } \\
\text { (hydrochloride) }\end{array}$} & 10 & $234.14846(100), 84.08066(62)$ \\
\hline & 20 & $84.08044(100)$ \\
\hline & 40 & $84.08049(100), 56.04966(21)$ \\
\hline \multirow{3}{*}{ MMAI (hydrochloride) } & 10 & $161.09616(100), 178.12288(10)$ \\
\hline & 20 & $\begin{array}{l}\text { 161.09609(100), 105.06997(55), } \\
\text { 146.07258(42), 131.07957(18), } \\
\text { 91.05413(14) }\end{array}$ \\
\hline & 40 & $\begin{array}{l}\text { 103.05423(100), 131.04918(51), } \\
91.05425(41), 77.03853(40), \\
115.05446(35)\end{array}$ \\
\hline \multirow[b]{3}{*}{ Etaqualone } & 10 & $265.13323(100)$ \\
\hline & 20 & $265.13338(100), 146.09647(11)$ \\
\hline & 40 & $\begin{array}{l}\text { 131.07315(100), 146.09651(97), } \\
\text { 118.06529(44), 130.06553(41), } \\
\text { 105.07006(33) }\end{array}$ \\
\hline \multirow{3}{*}{ Hydroxy Bupropion } & 10 & $238.09923(100)$ \\
\hline & 20 & $\begin{array}{l}\text { 238.09962(100), 167.04886(28), } \\
\text { 139.03111(20), 166.04196(19), } \\
131.07325(14)\end{array}$ \\
\hline & 40 & $\begin{array}{l}\text { 131.07319(100), 103.05449(96), } \\
130.06542(83), 139.03103(78), \\
166.04195(47)\end{array}$ \\
\hline \multirow[b]{3}{*}{ Levamisole } & 10 & $205.07886(100)$ \\
\hline & 20 & $205.07919(100), 178.06849(61)$ \\
\hline & 40 & $\begin{array}{l}91.05434(100), 123.02655(29), \\
\text { 128.06108(18), 117.06299(17), } \\
77.03874(16)\end{array}$ \\
\hline \multirow{3}{*}{ Loperamide } & 10 & $477.23075(100), 266.15462(34)$ \\
\hline & 20 & 266.15383(100), 477.23181(21) \\
\hline & 40 & $266.1543(100), 210.12803(36)$ \\
\hline \multirow{3}{*}{$\begin{array}{l}\text { N-Phenylacetyl-L-prolylglycine } \\
\text { ethyl ester }\end{array}$} & 10 & $\begin{array}{l}\text { 188.10741(100), 216.10233(82), } \\
70.06521(34)\end{array}$ \\
\hline & 20 & $70.06517(100), 188.10711(23)$ \\
\hline & 40 & $70.06517(100), 91.0545(22)$ \\
\hline
\end{tabular}




\begin{tabular}{|c|c|c|}
\hline \multirow{3}{*}{ Phenylpiracetam } & 10 & $174.09098(100), 202.08589(24)$ \\
\hline & 20 & $\begin{array}{l}\text { 174.09129(100), 145.06486(61), } \\
\text { 129.06994(46), 117.06928(13) }\end{array}$ \\
\hline & 40 & $\begin{array}{l}\text { 117.06935(100), 127.05441(83), } \\
91.0543(62), 115.05414(46), \\
129.07019(45)\end{array}$ \\
\hline \multirow{3}{*}{ Sildenafil } & 10 & $475.21351(100)$ \\
\hline & 20 & $475.2122(100)$ \\
\hline & 40 & $\begin{array}{l}58.06502(100), 283.11827(28), \\
100.09952(22), 99.09134(20.00593)\end{array}$ \\
\hline \multirow{3}{*}{ Sildenafil Citrate } & 10 & $475.2122(100)$ \\
\hline & 20 & $475.2122(100)$ \\
\hline & 40 & $\begin{array}{l}\text { 58.06513(100), 283.11895(22), } \\
\text { 100.0995(20), 99.09167(20) }\end{array}$ \\
\hline \multirow[b]{3}{*}{ Thiosildenafil } & 10 & $491.19018(100)$ \\
\hline & 20 & $491.1908(100)$ \\
\hline & 40 & $\begin{array}{l}\text { 58.06521(100), 299.09722(47), } \\
99.09142(29), 100.09958(24), \\
341.14216(14)\end{array}$ \\
\hline \multirow[b]{3}{*}{ Acetildenafil } & 10 & $467.27705(100)$ \\
\hline & 20 & $467.27718(100)$ \\
\hline & 40 & $\begin{array}{l}111.092(100), 127.12303(77), \\
297.13534(69), 84.08075(66), \\
72.08079(57)\end{array}$ \\
\hline \multirow{3}{*}{ Benzydamine } & 10 & $310.19154(100), 86.09635(48)$ \\
\hline & 20 & $86.09617(100)$ \\
\hline & 40 & $86.09651(100), 58.06544(76)$ \\
\hline
\end{tabular}




\section{MELANIE NICOLE ECKBERG}

2009-2013

B.S., Forensic Science

Virginia Commonwealth University

Richmond, Virginia

B.S., Chemistry

Virginia Commonwealth University

Richmond, Virginia

2012

2012

Undergraduate Researcher

Poklis Research Group

Virginia Commonwealth University

Richmond, Virginia

2013-2014

Intern

United States Postal Inspection Service

National Forensic Laboratory Services

Dulles, Virginia

Teaching Assistant

Florida International University

Miami, Florida

2013-2016

M.S., Forensic Science

Florida International University

Miami, Florida

2013-2018

Doctoral Student

Florida International University

Miami, Florida

2017-2018

Teaching Assistant

Florida International University

Miami, Florida

2012-present

2015-present

Society of Forensic Toxicologists, Associate Member American Academy of Forensic Scientists, Associate Member 


\section{PUBLICATIONS AND PRESENTATIONS}

Eckberg, M.N., Arroyo-Mora, L.E., DeCaprio, A.P. (2015) Expanded Compound Database and High Resolution MS/MS Spectral Library for the Detection of Designer Drugs by LC-QTOF-MS. International Forensic Research Institute Symposium, March 2015, Miami, FL.

Eckberg, M.N., Arroyo-Mora, L.E., DeCaprio, A.P. (2015) Expanded Compound Database and High Resolution MS/MS Spectral Library for the Detection of Designer Drugs by LC-QTOF-MS. Society of Forensic Toxicologists Annual Meeting, October 2015, Atlanta, GA.

Arroyo-Mora, L.E., DeCaprio, A.P., Gluodenis, T., Broomes, A.M., Eckberg, M.N., Kimble, A.N., Seither, J.Z. (2016) Comprehensive Analytical Tools for the Identification of Emerging Drugs of Abuse. National Institute for Standards and Technology (NIST) Trace Evidence Data Workshop, 2016, Gaithersburg, MD.

Eckberg, M.N., Arroyo-Mora, L.E., Stoll, D.R., DeCaprio, A.P. (2017) The Separation of Chemically Similar and Isobaric Novel Psychoactive Substances (NPS) Using TwoDimensional Liquid Chromatography (2D-LC). American Academy of Forensic Sciences Annual Meeting, February 2017, New Orleans, LA.

Eckberg, M.N., Arroyo-Mora, L.E., Stoll, D.R., DeCaprio, A.P. (2017) The Separation of Chemically Similar and Isobaric Novel Psychoactive Substances (NPS) Using TwoDimensional Liquid Chromatography (2D-LC). International Forensic Research Institute Symposium, March 2017, Miami, FL.

Eckberg, M.N., Arroyo-Mora, L.E., DeCaprio, A.P. (2018) Development of a High Resolution Mass Spectrometry (HRMS) Library and Method Validation for Screening and Confirmation of 800+ Novel Psychoactive Substances (NPS) by Liquid Chromatography/Quadrupole Time-of-Flight/Mass Spectrometry (LC/QTOF/MS). American Academy of Forensic Sciences Annual Meeting, February 2018. Seattle, WA.

Eckberg, M.N., Arroyo-Mora, L.E., DeCaprio, A.P. (2018) Development of a High Resolution Mass Spectrometry (HRMS) Library and Method Validation for Screening and Confirmation of 800+ Novel Psychoactive Substances (NPS) by Liquid Chromatography/Quadrupole Time-of-Flight/Mass Spectrometry (LC/QTOF/MS). International Forensic Research Institute Symposium, May 2018, Miami, Florida.

Eckberg, M.N., Arroyo-Mora, L.E., Stoll, D.R., DeCaprio, A.P.: Separation and Identification of Isomeric and Structurally Related Synthetic Cannabinoids Using TwoDimensional Liquid Chromatography and High Resolution Mass Spectrometry. (In print). 